\title{
The VT GAL4, LexA, and split-GAL4 driver line collections for targeted expression in the
}

\section{Drosophila nervous system}

\author{
Laszlo Tirian $^{1}$ and Barry J. Dickson ${ }^{1,2,3}$ \\ ${ }^{1}$ Research Institute of Molecular Pathology, Campus-Vienna-Biocenter 1, A-1030 Vienna, \\ Austria \\ 2 Janelia Research Campus, HHMI, 19700 Helix Drive, Ashburn VA, 21407, U.S.A. \\ ${ }^{3}$ Correspondence: dicksonb@janelia.hhmi.org
}

\begin{abstract}
In studying the cellular interactions within complex tissues, it is extremely valuable to be able to reproducibly and flexibly target transgene expression to restricted subsets of cells. This approach is particularly valuable in studying the nervous system, with its bewildering diversity of neuronal cell types. We report here the generation of over 18,000 driver lines (the VT collection) that exploit the GAL4, LexA, and split-GAL4 systems to express transgenes in distinct and highly specific cell types in Drosophila. We document the expression patterns of over 14,000 of these lines in the adult male brain.
\end{abstract}

\section{Results and Discussion}

The GAL4/UAS system (Brand and Perrimon, 1993) has proven to be a robust and versatile method for targeted transgene expression in Drosophila. Large collections of GAL4 lines have previously been generated (Gohl et al., 2011; Hayashi et al., 2002; Jenett et al., 2012). Complementing these resources, we generated a total of 8,166 enhancer-GAL4 lines (the "VT" or "Vienna Tiles" lines) using the strategy described by Pfeiffer et al. (2008). Each of these VT GAL4 driver lines contains a predicted $\sim 2 \mathrm{~kb}$ enhancer region, or in some cases a promoter region, cloned into the $\mathrm{pBPGUw}$ vector or its promoterless equivalent $\mathrm{pBPGw}$. These GAL4 driver constructs were inserted by site-specific recombination into the attP2 landing site on chromosome arm 3L (Groth et al., 2004; Table 1).

The expression pattern of 7,705 VT GAL4 driver lines in the embryo has been determined using in situ hybridization to detect the GAL4 mRNA (Kvon et al., 2014). In this work, we used a $U A S$ $m C D 8-G F P$ reporter and confocal imaging to reveal the expression patterns of 8,081 VT GAL4 lines in confocal images of adult male brains. Most of these lines showed reproducible expression in restricted subsets of neuronal cell types. Approximately 3000 lines that were considered most useful for neurobiological experiments were selected for recloning into LexA and split-GAL4 vectors.

The LexA/lexAop system (Lai and Lee, 2006) can be used in conjunction with the GAL4/UAS system to independently target distinct transgenes to two distinct cell subsets. In neurobiological experiments, for example, a researcher often wishes to optogenetically activate one population of neurons while silencing or imaging activity in another population. To enable such experiments, 
we transferred many of the more useful enhancers and promoters into LexA vectors (Pfeiffer et al., 2010), generating a total of 3,624 VT LexA lines (Table 1). We used vectors coding for LexA fusion proteins in which the LexA DNA-binding domain is coupled to either a GAL4 or a human p65 activation domain (Pfeiffer et al., 2010), thus providing both GAL80-suppressible and GAL80-insensitive LexA variants. These VT LexA constructs were inserted into either attP2 (Groth et al., 2004), attP40 on 2L (Markstein et al., 2008), or both sites (Table 1). Insertions in the attP2 site more reliably recapitulate the expression pattern of the original VT GAL4 lines, but are subject to transvection when paired with VT GAL4 lines also in attP2 (Mellert and Truman, 2012). The insertions in attP40 avoid transvection with any enhancer-GAL4 lines in attP2, with which they can also be combined in doubly homozygous stocks. The expression patterns of 3,054 VT LexA lines in adult male brains were determined using a lexAop-myr-GFP reporter (Table 1).

The VT GAL4 and LexA driver lines, like the analogous lines generated at Janelia (Jenett et al., 2012), rarely restrict expression to a single neuronal cell type. We therefore inserted the most useful enhancers and promoters into the ZpGAL4DBD and p65ADZp split-GAL4 vectors for intersectional targeting (Luan et al., 2006; Pfeiffer et al., 2010). In this system, neither the ZpGAL4DBD nor the p65ADZp hemidriver alone is sufficient to drive expression of a UAS transgene; only when the two are coexpressed in the same cell is a functional GAL4 reconstituted to drive transgene expression. We generated 2,736 VT ZpGAL4DBD lines, all inserted in attP2, and 2,826 VT p65ADZp lines, approximately one third of which were inserted in attP2 and the rest in attP40 (Table 1). To determine the expression patterns of individual hemidriver lines, they were combined with a pan-neuronal hemidriver (either elav-p65ADZp or elav-ZpGAL4DBD) and $U A S-m C D B 8-G F P$ for confocal imaging of male brains. Images were acquired for 1,488 VT ZpGAL4DBD lines and 2,012 VT p65ADZp lines (Table 1).

Our collections of VT GAL4, LexA, and split-GAL4 driver lines complement similar collections generated at Janelia (Dionne et al., 2017; Jenett et al., 2012), with which they are fully compatible. In our experience, the many thousand split-GAL4 hemidriver lines available in these collections ensure that, more often than not, multiple independent intersections can be obtained for any desired neuronal cell type. The critical factor in the success of any such endeavor is the ability to efficiently identify the useful intersections from the several million potential DBD and AD combinations, a task greatly facilitated by the image databases of parental GAL4 lines and, in the case of the VT lines, also the split-GAL4 hemidriver lines.

Our data set of 41,492 confocal images of male brains from 14,635 lines can be accessed online at Virtual Fly Brain (www.virtualflybrain.org) (Milyaev et al., 2012). Many of the VT GAL4 and LexA lines have also been independently imaged in female brains and ventral nerve cords at Janelia (www.janelia.org/gal4-gen1). Approximately 1000 of the most useful VT GAL4 lines are available from the Vienna Drosophila Resource Center (www.vdrc.at). Almost all of the splitGAL4 hemidriver lines, together with a small number of additional VT hemidriver lines subsequently generated at Janelia, are available from the Bloomington Drosophila Stock Center (Table 2, www.flystocks.bio.indiana.edu). 


\section{Acknowledgements}

We thank the following for their contributions to the generation of the VT lines and/or images: Salil Bidaye, Luisa Deszcz, Balazs Erdi, Michaela Fellner, Angela Graf, Attila György, László Hunor Hajdu, Martin Kinberg, Christopher Masser, Magdalena Mosiolek, Thomas Peterbauer, Elena Popowich, Anton Russell, Alex Stark, Johanna Trieb, Sandor Urmosi-Incze, Stefanie Wandl, Yang Wu, Amina Zankel, and Svetlana Zorinyants. We also thank Katja Bühler and colleagues at VRVis for the BrainGazer software package used to manage the imaging pipeline and Karen Hibbard, Evgeny Kvon, Todd Laverty, Lisa Meadows and Cahir O'Kane for comments on the manuscript. Research at the IMP is supported by Boehringer Ingelheim $\mathrm{GmbH}$.

\section{Methods}

\section{VT line generation}

The non-coding, non-repetitve regions of the Drosophila genome were tiled into $\sim 2 \mathrm{~kb}$ fragments (Kvon et al., 2014) and individual tiles ranked for cloning according to various criteria considered predictive of a neuronal enhancer or promoter, such as proximity to predicted or known neuronal genes and the presence of conserved cis-regulatory motifs. Selected tiles were amplified by PCR from $w^{1118}$ genomic DNA and cloned into the entry and transformation vectors as previously described (Pfeiffer et al., 2008; 2010). Germline transformation of attP2 or attP40 hosts (Groth et al., 2004) was performed either in house or by BestGene Inc. (www.thebestgene.com).

\section{Immunohistochemistry and confocal imaging}

Flies were dissected, stained and registered to the T1 template as described in (Yu et al., 2010). In brief, brains were dissected in phosphate buffered saline (PBS), fixed in $4 \%$ paraformaldehyde in PBS with $0.1 \%$ TritionX100 and subsequently blocked in $10 \%$ normal goat serum (Gibco Life Technologies). Brains were incubated in primary and secondary antibodies for 36 hours at $4^{\circ} \mathrm{C}$ and washed in PBS with $0.3 \%$ TritionX100 for 48 hours at $4^{\circ} \mathrm{C}$. Fly brains were mounted in Vectashield (Vector Laboratories). The following antibodies were used: rabbit polyclonal anti-GFP (1:5000, TP401, Torrey Pines) and mouse monoclonal anti-Bruchpilot (1:20, nc82, Developmental Studies Hybridoma Bank) primary, and Alexa Fluor 488 and 568 conjugated secondary antibodies (1:500 to 1:1000, Invitrogen Life Technologies). Images were acquired with Zeiss LSM700 confocal microscopes equipped with $25 \mathrm{x} / 0.8$ plan multi-immersion objectives in a single-track mode with $0.5 \times 0.5 \times 1 \mu \mathrm{m}$ voxel size. Images were aligned by nonrigid registration using the $\mathrm{nc} 82$ staining pattern as reference channel. 


\section{References}

Brand, A.H., and Perrimon, N. (1993). Targeted gene expression as a means of altering cell fates and generating dominant phenotypes. Development 118, 401-415.

Dionne, H., Hibbard, K.L., Cavallaro, A., Kao, J.-C., and Rubin, G.M. (2017). Genetic reagents for making split-GAL4 lines in Drosophila. bioRxiv 197509.

Gohl, D.M., Silies, M.A., Gao, X.J., Bhalerao, S., Luongo, F.J., Lin, C.C., Potter, C.J., and Clandinin, T.R. (2011). A versatile in vivo system for directed dissection of gene expression patterns. Nature Methods 8, 231-237.

Groth, A.C., Fish, M., Nusse, R., and Calos, M.P. (2004). Construction of transgenic Drosophila by using the site-specific integrase from phage phiC31. Genetics 166, 1775-1782.

Hayashi, S., Ito, K., Sado, Y., Taniguchi, M., Akimoto, A., Takeuchi, H., Aigaki, T., Matsuzaki, F., Nakagoshi, H., Tanimura, T., et al. (2002). GETDB, a database compiling expression patterns and molecular locations of a collection of Gal4 enhancer traps. Genesis 34, 58-61.

Jenett, A., Rubin, G.M., Ngo, T.T., Shepherd, D., Murphy, C., Dionne, H., Pfeiffer, B.D., Cavallaro, A., Hall, D., Jeter, J., et al. (2012). A GAL4-driver line resource for Drosophila neurobiology. Cell Reports 2, 991-1001.

Kvon, E.Z., Kazmar, T., Stampfel, G., Yanez-Cuna, J.O., Pagani, M., Schernhuber, K., Dickson, B.J., and Stark, A. (2014). Genome-scale functional characterization of Drosophila developmental enhancers in vivo. Nature 512, 91-95.

Lai, S.L., and Lee, T. (2006). Genetic mosaic with dual binary transcription systems in Drosophila. Nat Neurosci 9, 703-709.

Luan, H., Peabody, N.C., Vinson, C.R., and White, B.H. (2006). Refined spatial manipulation of neuronal function by combinatorial restriction of transgene expression. Neuron 52, 425-436.

Markstein, M., Pitsouli, C., Villalta, C., Celniker, S.E., and Perrimon, N. (2008). Exploiting position effects and the gypsy retrovirus insulator to engineer precisely expressed transgenes. Nat Genet 40, 476-483.

Mellert, D.J., and Truman, J.W. (2012). Transvection is common throughout the Drosophila genome. Genetics 191, 1129-1141.

Milyaev, N., Osumi-Sutherland, D., Reeve, S., Burton, N., Baldock, R.A., and Armstrong, D. (2012). The Virtual Fly Brain browser and query interface. Bioinformatics 28, 411-415.

Pfeiffer, B.D., Jenett, A., Hammonds, A.S., Ngo, T.T., Misra, S., Murphy, C., Scully, A., Carlson, J.W., Wan, K.H., Laverty, T.R., et al. (2008). Tools for neuroanatomy and neurogenetics in Drosophila. Proc Natl Acad Sci U S A 105, 9715-9720. 
Pfeiffer, B.D., Ngo, T.T., Hibbard, K.L., Murphy, C., Jenett, A., Truman, J.W., and Rubin, G.M. (2010). Refinement of tools for targeted gene expression in Drosophila. Genetics 186, 735-755.

Yu, J.Y., Kanai, M.I., Demir, E., Jefferis, G.S., and Dickson, B.J. (2010). Cellular organization of the neural circuit that drives Drosophila courtship behavior. Current biology 20, 1602-1614. 
Table 1. Numbers of VT lines generated and images.

\begin{tabular}{|l|c|c|}
\hline Driver and landing site & Lines & Imaged lines \\
\hline GAL4 in attP2 & 8166 & 8081 \\
LexAGAD in attP2 & 446 & 438 \\
LexAp65 in attP2 & 658 & 657 \\
LexAGAD in attP40 & 2240 & 1694 \\
LexAp65 in attP40 & 280 & 265 \\
ZpGDBD in attP2 & 2736 & 1488 \\
p65ADZp in attP2 & 732 & 728 \\
p65ADZp in attP40 & 2094 & 1284 \\
\hline
\end{tabular}

\section{Table 2. VT lines and images.}

CRM (cis-regulatory module) type: E, enhancer; L, promoter facing leftward, R, promoter facing rightward.

Stock availability: BDSC, Bloomington Drosophila Stock Center; VDRC, Vienna Drosophila Resource Center; JRC, Janelia Research Campus; unavailable, stock no longer publically maintained. Availability according to respective stocklists on Oct 1, 2017. 


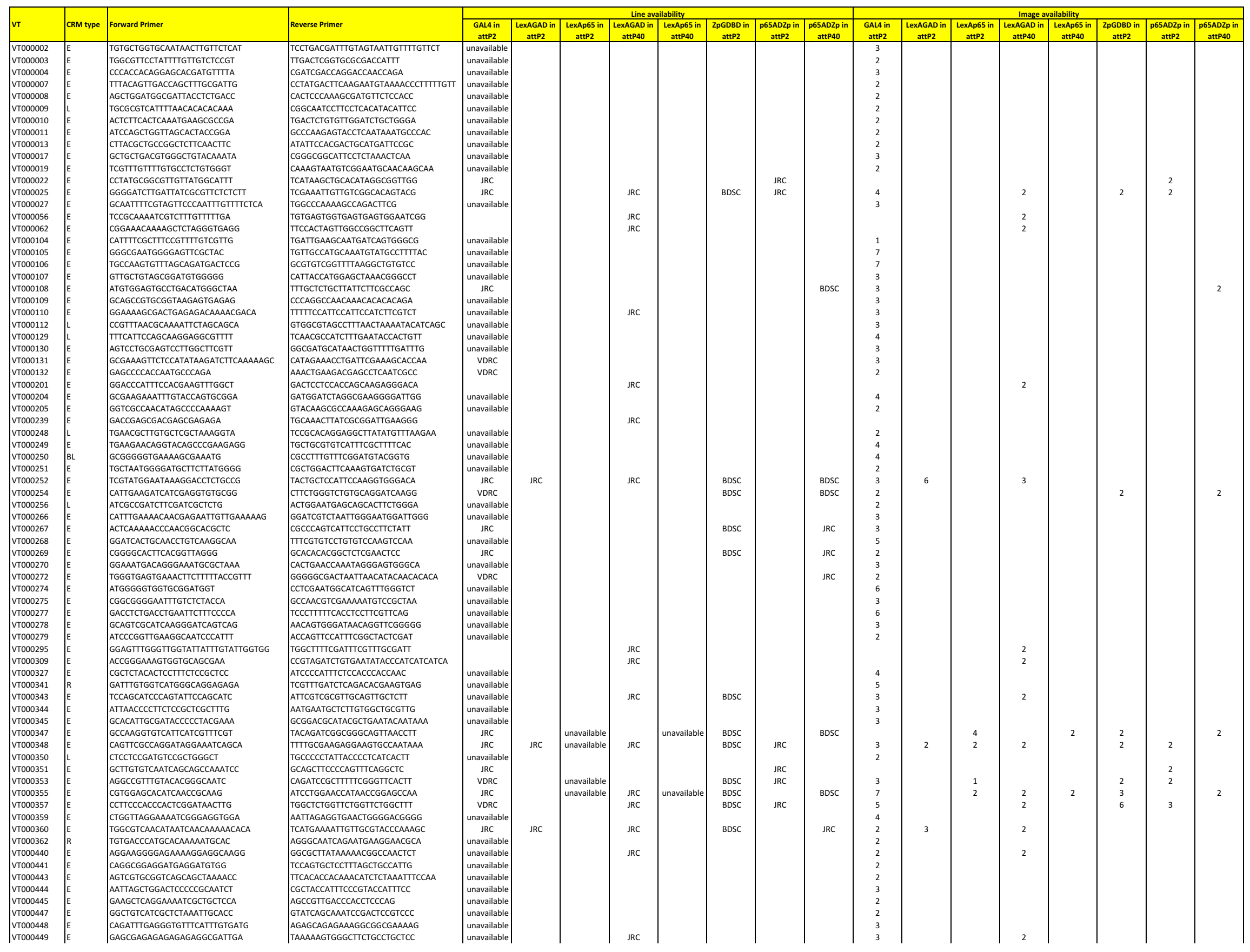




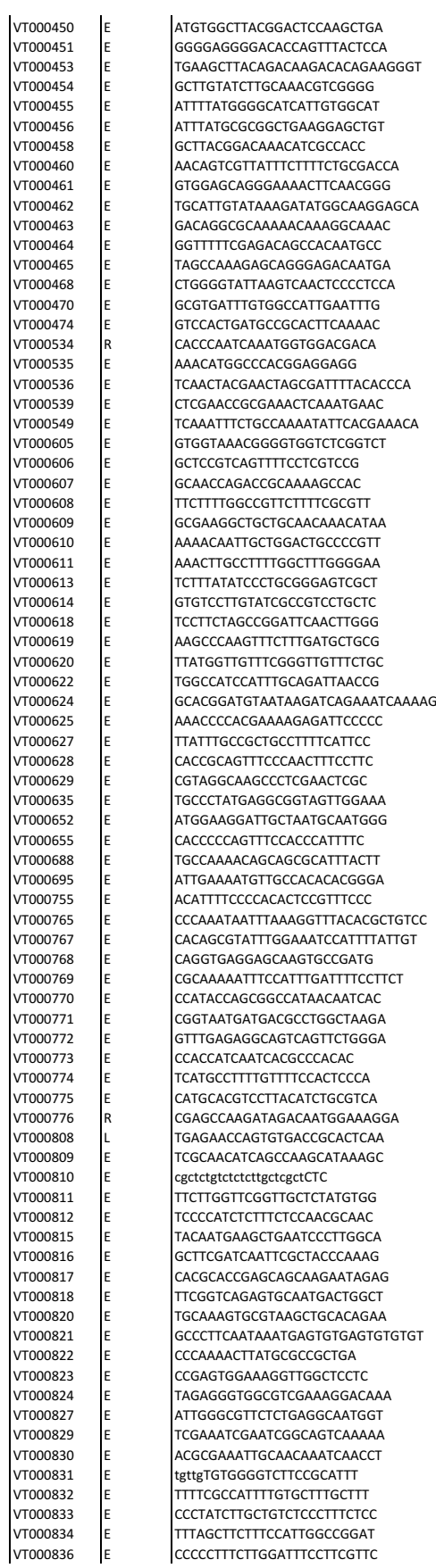

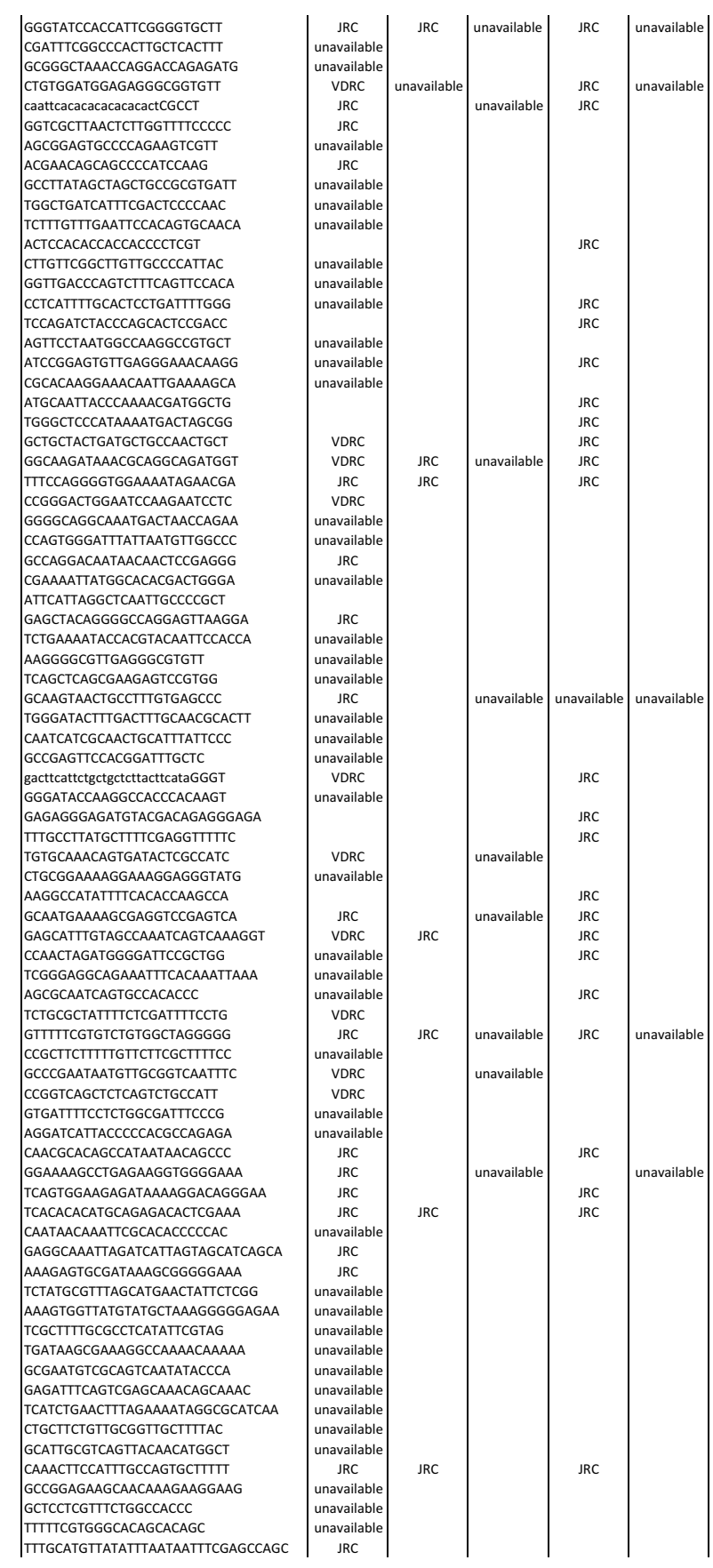
AAACCAGGACCAGAGAT GGAAAATACCACGTACAATTCCACC CAGCTCAGCGAAGAGTCCGTGG GgataCTTGACTTGCAACGCACT GATCATCGCAACTGCATTTATTCCC
SCCGAGTCCACGGATTTCTC GGATACCAGGGCCACCCACAAG GTGATTRCTCTGGGGATTCCCG GAAAAGCCTGAGAAGGTGGGGAAA ACACACATGCAGAGACACTCGAAA

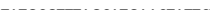
(TCGITTCGGCCACCC

ITTGCATGTATATTAATAATTCGAGCCAGC

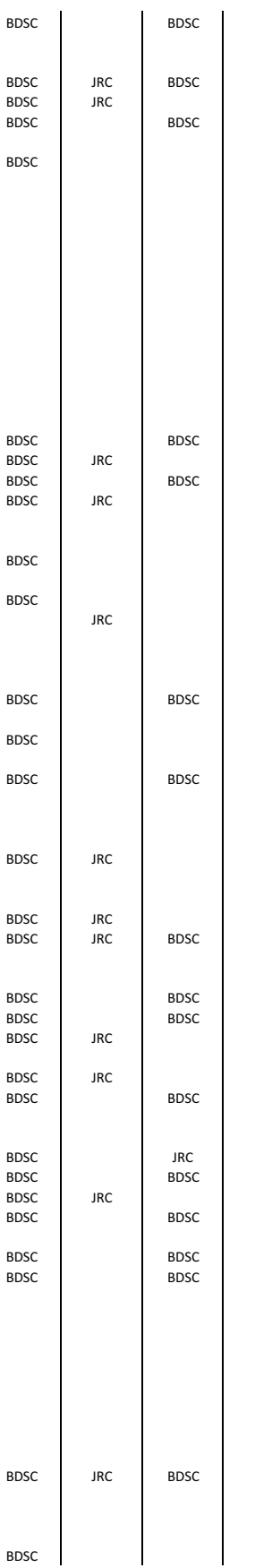

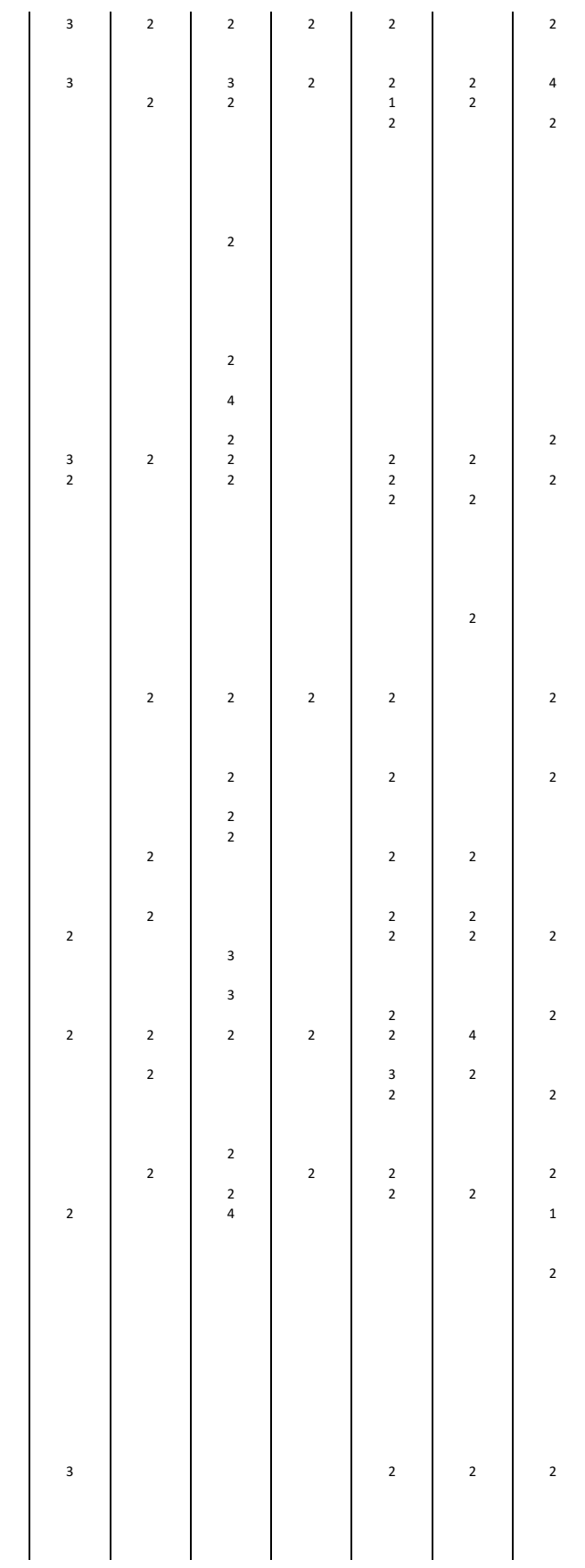




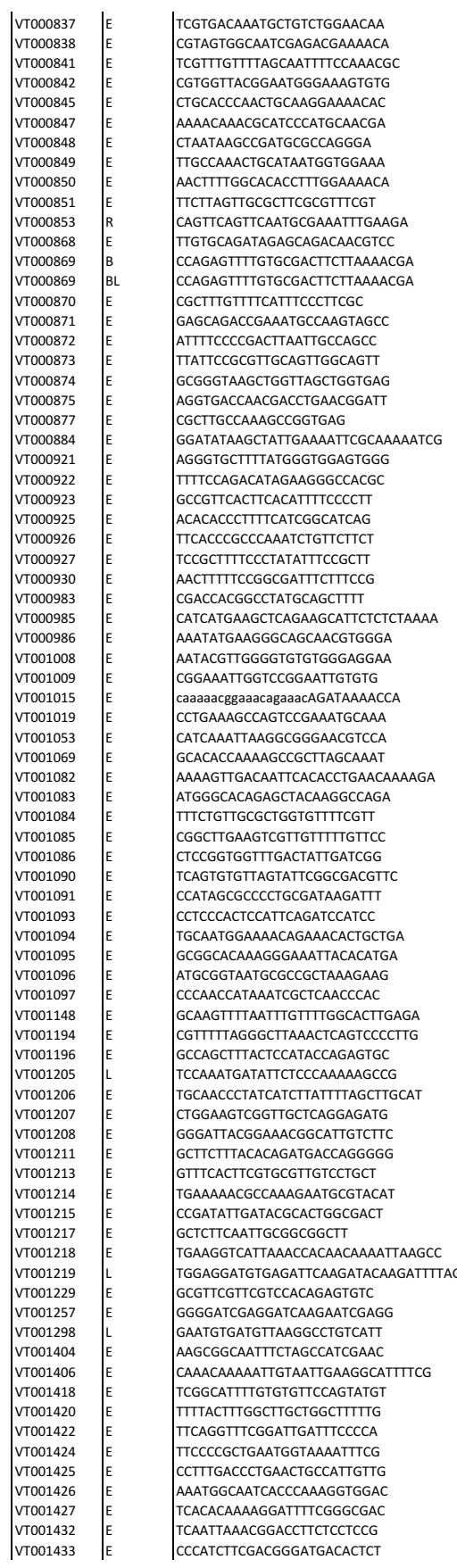

GAAagaAagccGagaAaAGGgGGAa

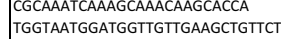
GAGCCCAGTGAGTGGTGGTTGGAAG GCTCAGGTATCTGGTGGTAACCTATCTGCC GGTCAAATCAAACGCATTGCACGA GCTGTCCTACGCTCCGCCTG GCTGTGTTCGCTTTTCGCTTTCCT ITTGGTAAGGGGTGCTAGAGCTGGA AACCAACTGACTACGCGAAGGGAAA ACCAACTGACTACGCGAAGGGAAA GTCTATCCCTATGCCCGGCCAGAAA TGTGCGGAGAATGCCTTAATTGTTG TICCAGTGCCTGCTITAACATTC ACTGCTGCTGGCATCGCTGTTC CCAGACGACTCAAAATAGCTGGCCC AGTTGCCAAAATTGGGCAAGAACAG

TGCAGCATCAGTAGGTGGAGGAATATTCA GGGGGGGGAGCTATCTTGAGCTATT GCTTTCGCAGTCAAGTCATTGTTATGGTT GCATTACATCCCTGTATCTCAGCCCC
TTGCTACCCACTGGCTITCCTTG TIATCGCTGGACACCGGCATTCAC GTTACCTGCTGCGGCCCTGCT AGGTAGCCGATCCTTCAGGGCA TCGGGTGCTITCCTCATTACTC TGCACTTGAGATTGACCTGAAACTACCA GCATTAAGCTGTGAACTITTAGGCAAG

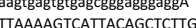
matgagtcagcTag Togcagacca ICCAATTATGTTAAGCTCCATTGCTTA ATCCCACGATCCCACACACGAAAAC AGGAACCACATGAACTCGAAACCC TGGGGGAGGTGGGAGGTGAG

CGCAATGTGTTGAAGGAGCTGGAA ITTATGGGGATCTTGGGAGTGGTTT CAGTCAAAAAGAAACTGCCGTGCTGG GTGGAGAAGGAGTCGGGGAATGGA ACGTGCAGAAAAGAGGCAGTCAAAA GCTGCTCAAATGCAGCAAATAGCAAA

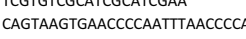
AGCACAAGCTATGTGGACGTTCTTCTII TCCTTCCTTTCGCCTTCGTCGTG IGAGAAATGTGAAATTGTTCCAGTTCC GCCGATGAAATTCCTCCTCCTCAA GACTCCGCTGGCCTCCCAGTT TGCCACCCATCATCGGTGTACACTT GTACTTGTCGTCTGGGGGGGGG TCTATGCGGGGGCCAACAACTC TTGGCTGGCACTATTTCTCCCCAA GTTGAGGGAAGGAGGCTACGAGG TITGACACCGACTCGACTTCCCC AAGCGTGAAGATACATTATAGGGACAAA GGCATCATCATGACTCGTGCAATA GGgTCACTATGCTGGTTCAACTTT ITTTACACACCCAGTTCAAAATGCAAATA TGGCTGAGAGGATAAGTGGTTTTGGTG GGTGCTGCAGCTAAaGAaAAACTAATCTC CGGGTTGGCTCTCGGGTTT GGATGGGGCTGGTGCTAAGATG
GCAGGTGATTGGATGAGGTGGC
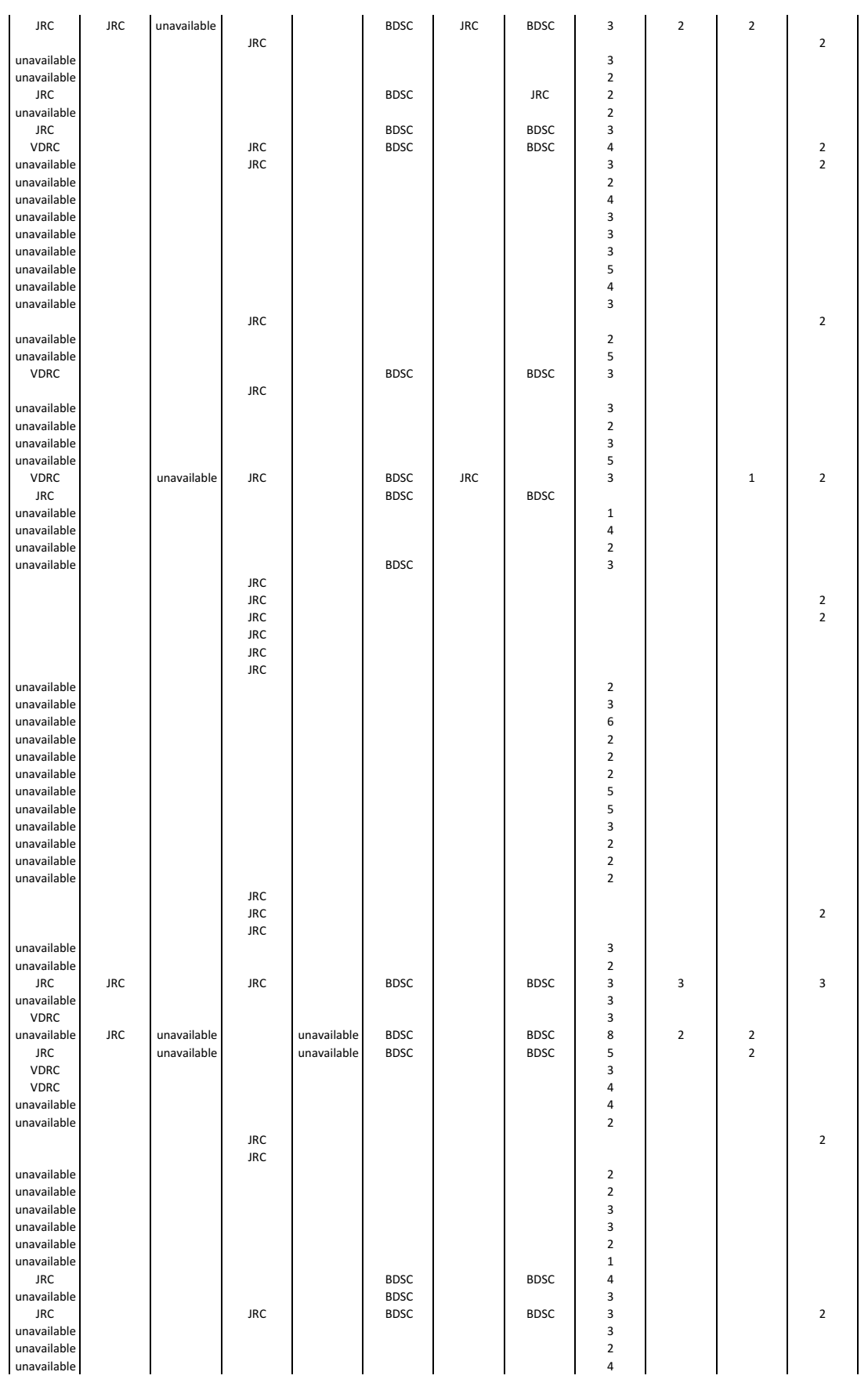

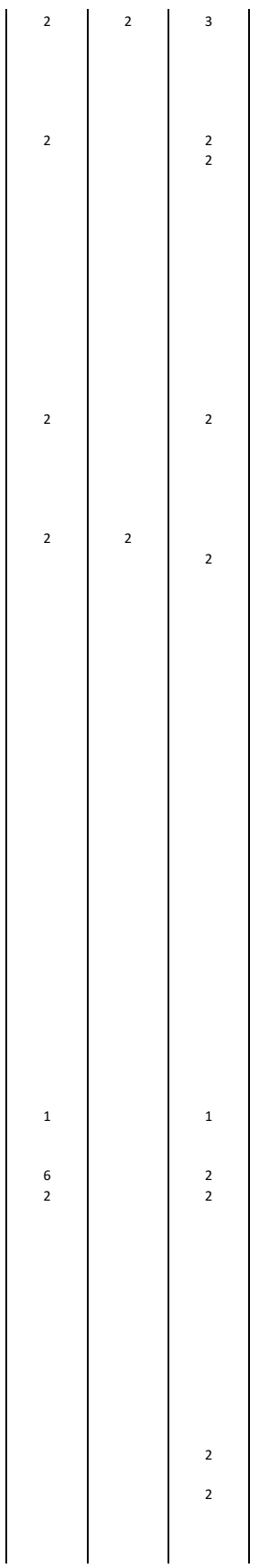




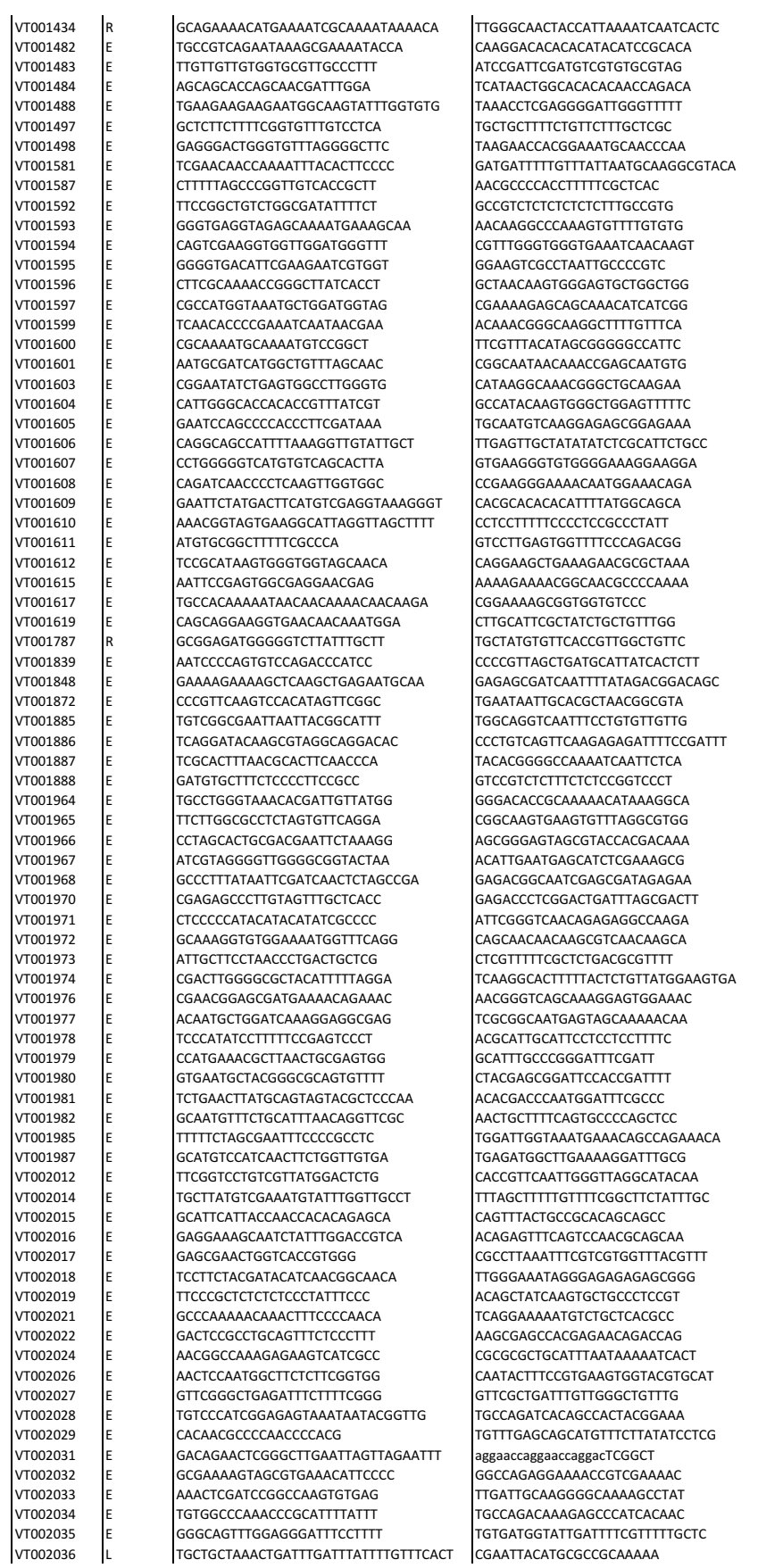

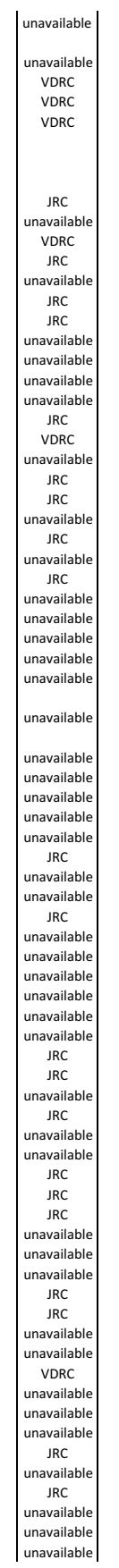
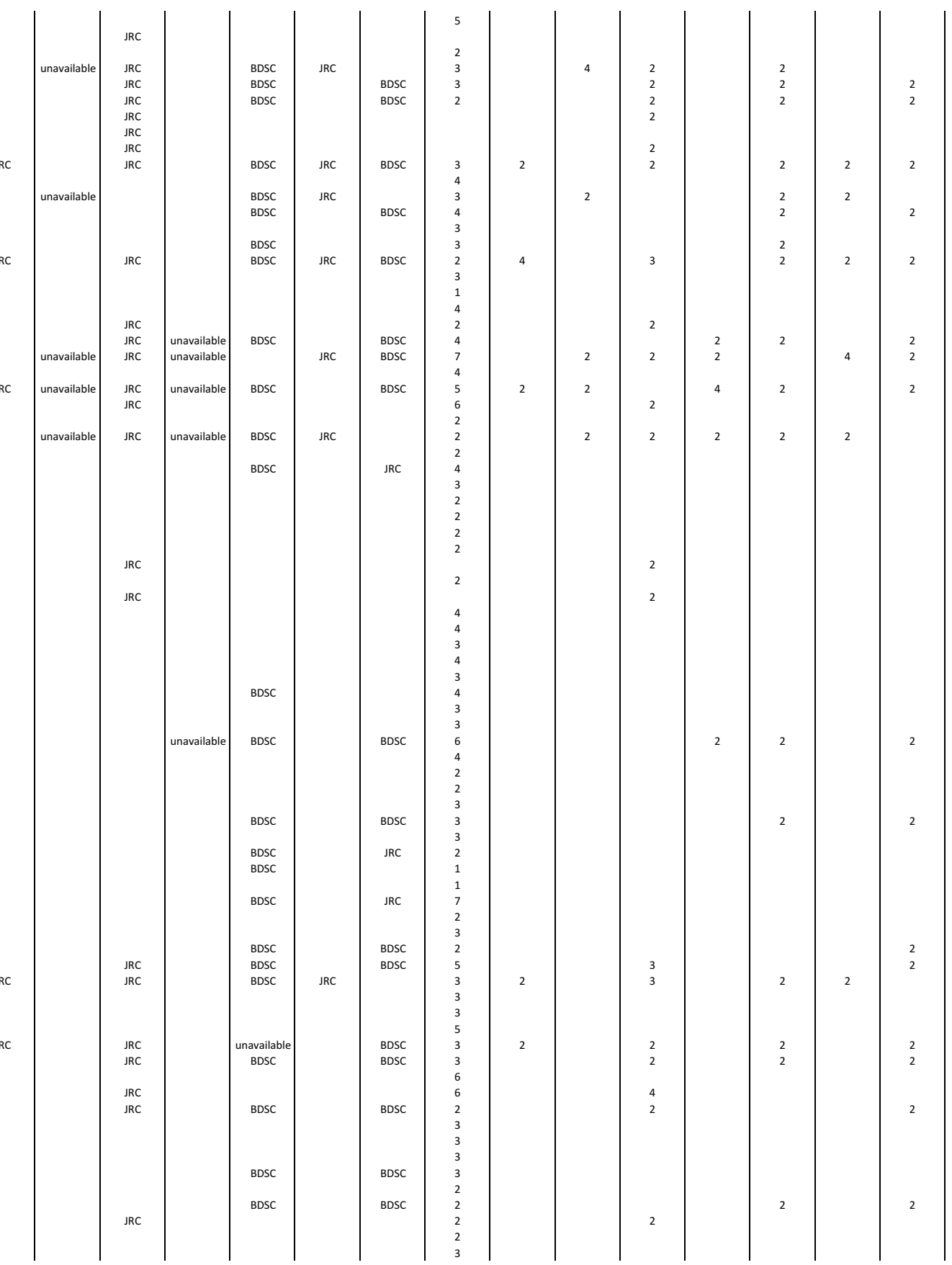


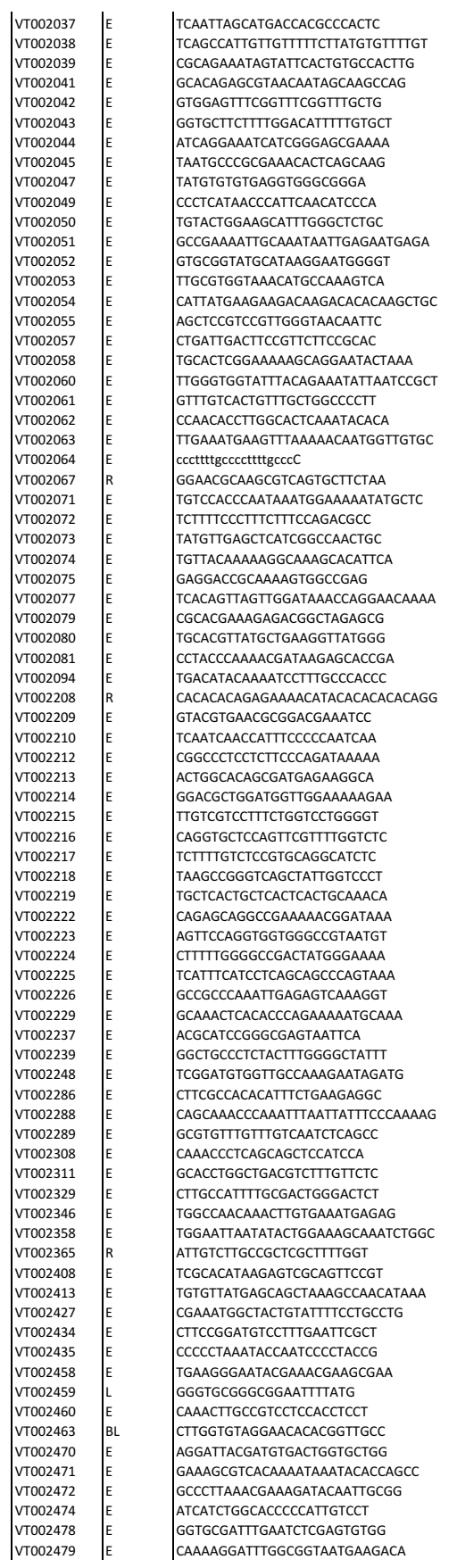

CAAGTCCAAGTTGCCCAGCTTCCA GCGGCTTTTACCCACTCCCCA GTGCAGTATTCGCAGTTGCACTT AATTGCATAGCGAATGGATTCT GACGGCAGCAGCTAAAAGAAGGG
GCGITATTGAAACGAAATACATGCCC GTGCTAAAGGGTTCTCCCCAAAA GCATACAATTAACAGCACACCGAACTCC GGGCATTGTTCGATTCACATTCACA GAAGTIAGAATCTCGGAGGGTGGG IGCTGCTGGAGAGGAAATAACCAAC ACTTITGGGCTGCCTTATTCCCCT CAAACATATGTGCACAAACGAAAGCC CAAGCTTACAAAGCCAGGAAAA IGGGACACACTTTAGTTGGCATC ICATTACCCAATTCCCAAGGCTCTC TGGCTGGGTTAAAACTAAAACTGTAGC
ATGCGCGGCTACTTCATTIACACA GCACTAATCTCTGTAGAAAGTCGGTCCA IGCGGGTAAAAATTGGGTAAGGATTTCA CTGACTCTGGCACTAACTTTCGG GGGCACAGGATTAATACTCGGATGAGG ICAGGGAAATATAAAGTTGCAAGTGAA CGCTGCACTGCGAAAATAAATCAAG MAATTCTTGCGGGCCACTTGAGA ICACGTACGCCGTGGTGGAACAA SCGGACATTGAGCATTTGATTGT TTCCATCTCGGTTGCTTCGGCT CCATCCTGCACAGGAAGAAAGAACC CGGTGAGAGAGTGGGGAACAGAATA CATCCCCGCCCCCTCCATGTATC GGTAGTTCTTATATTCGITACTGCGTGG TAAGCATGCAGAAGAGGCTGGGAAA CCACCACCCCAACCCAACCA - ATTITCAAACCTACGGGTGCGCC GTAAAACTCGCATGGCAACCAGGA GATGAGCTGACGGTGGGAATGGG GGTAGAACTTGACGATATGCGCCCG ICCAAGGGGAGGAAGATATGGGG sctttgctttgctgtggCCTCTTTC GCAGCCCGACATGAAATCAAGGGTG GGGCCTACTCTGAGGTGTAGGTT ggttetTCCAGGTCTITGGCCC AACCTGCACATGCGATAAGGCAGA ITCGCACACGGTGATTGGATCAGT
TCTGGGCATATAGCAGCATTCACTTCA
AAGAGGGTGGGGAAGGGGCTGGG GCTGATGGGATTTTAATTAGACACTCACT TCTTACTGGAGATATTTGTCATCGACTTI TGAAGAGCCCGCTTTTAACTGGC ATAAAGCACTCATTICCGGCeC CCCACCCATTTCGGGCTATAAAAC AATTGAAATTGCAGCGGGGAGTAAG GCAGCAGTTGTCCAGGTIACAAATCCA
GACTCGGATGTTAGGTCTAGTTAAAG CCGCGITTACTTCGATCTGGCACT AGCTAACGATGGGTTGCGCTCC GGGTGGAAATGCAAGATACCCTGT GGAGGGCAGCTCGHCTATGCTCC CATCGCTTGAAATCGCTTCCCTTG GTTGCTGGTAGCGGGGAAAAAGGA
GCCCTCGGACAGTTCACTCGAT

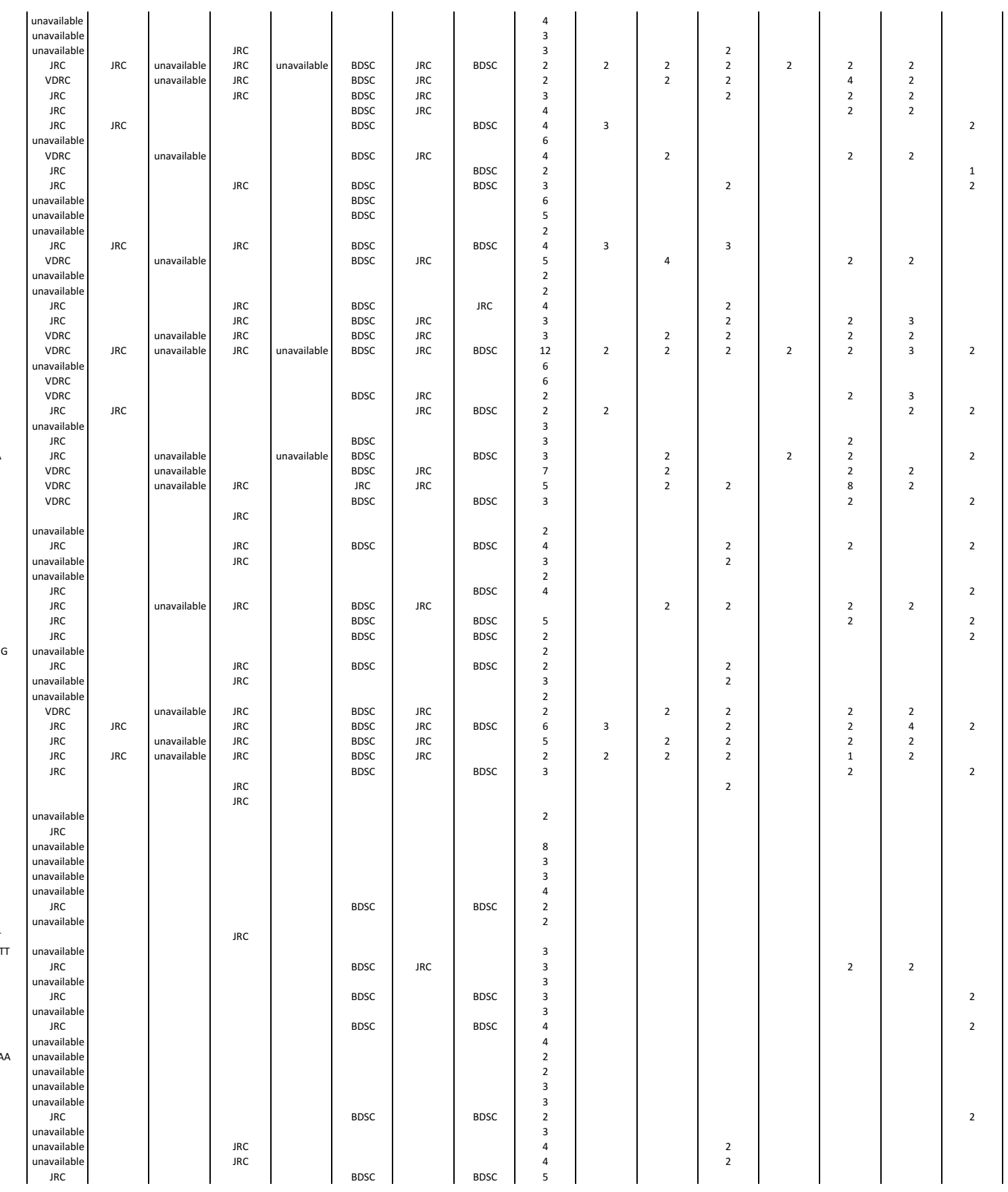
\begin{tabular}{|l|l|}
\hline JRC & AGCCTCGGACAGTTCCACTCGAT
\end{tabular} 


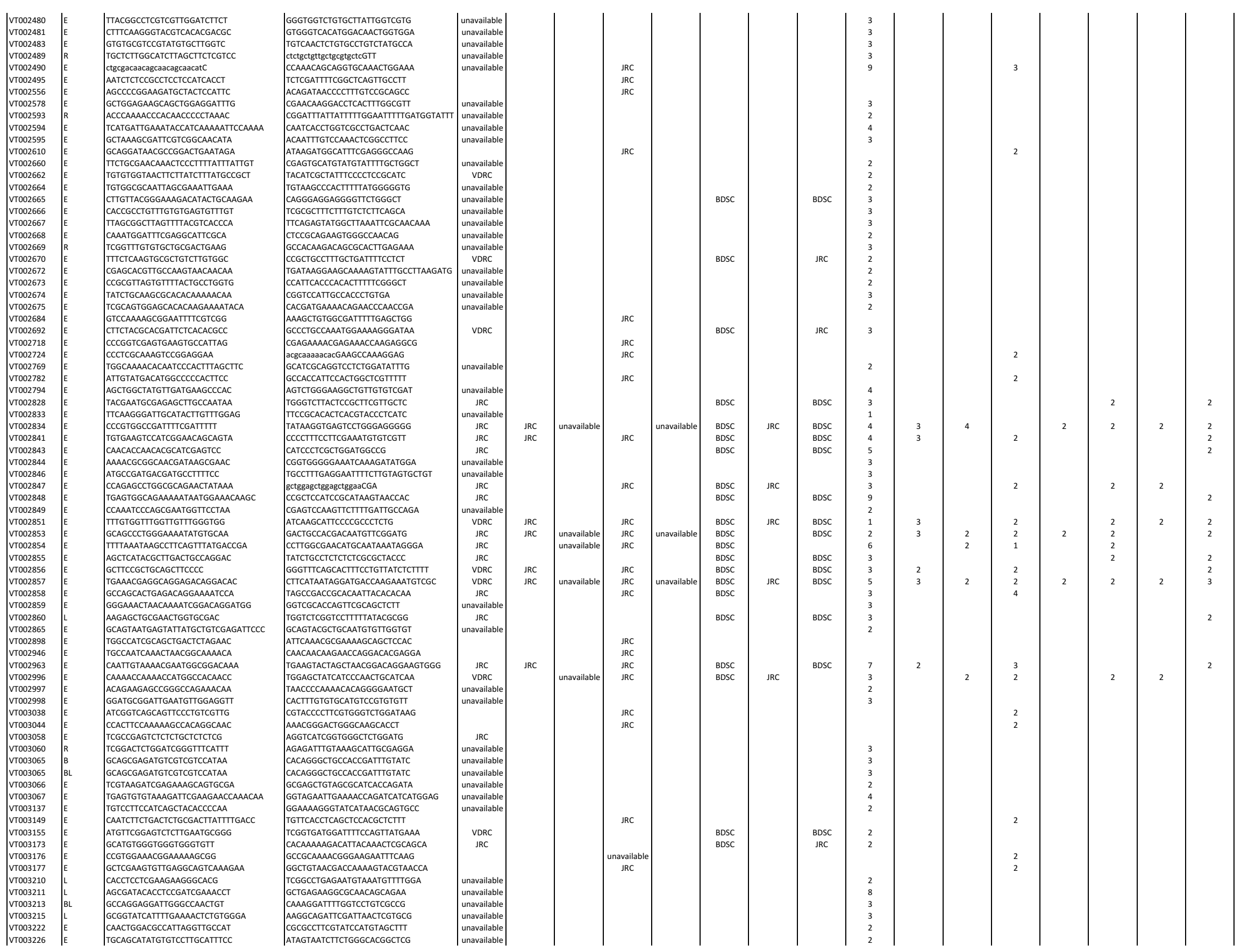




\begin{tabular}{|c|c|c|}
\hline \multicolumn{2}{|c|}{ 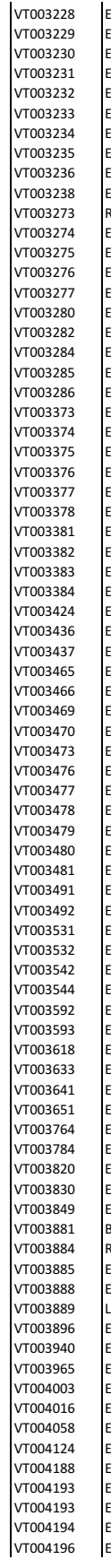 } & 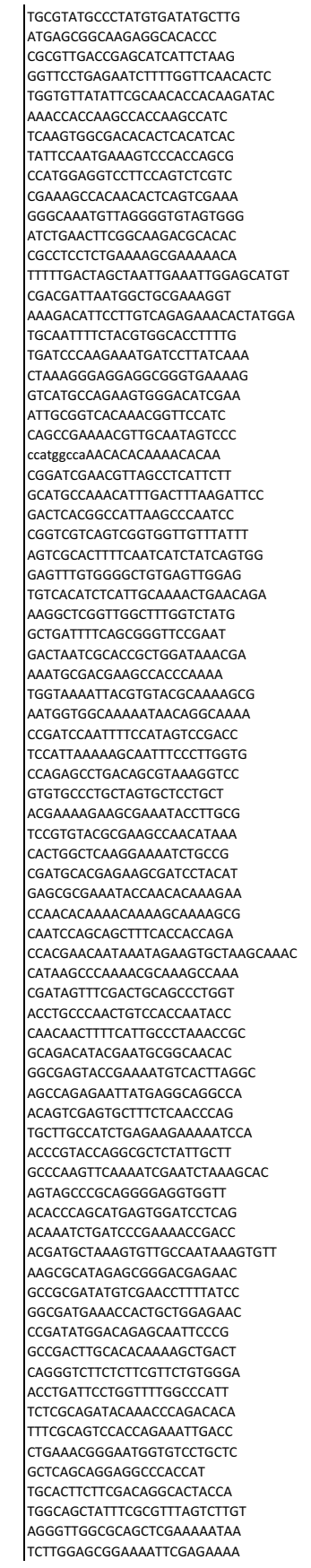 \\
\hline
\end{tabular}

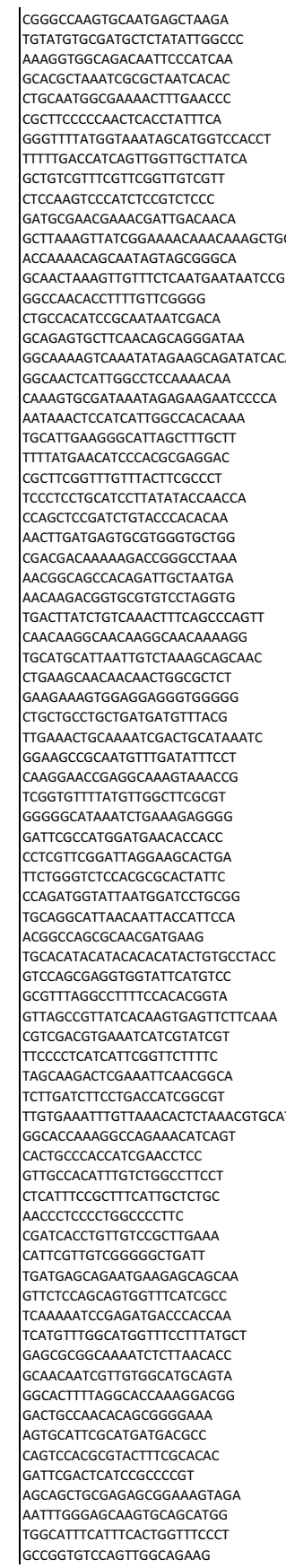
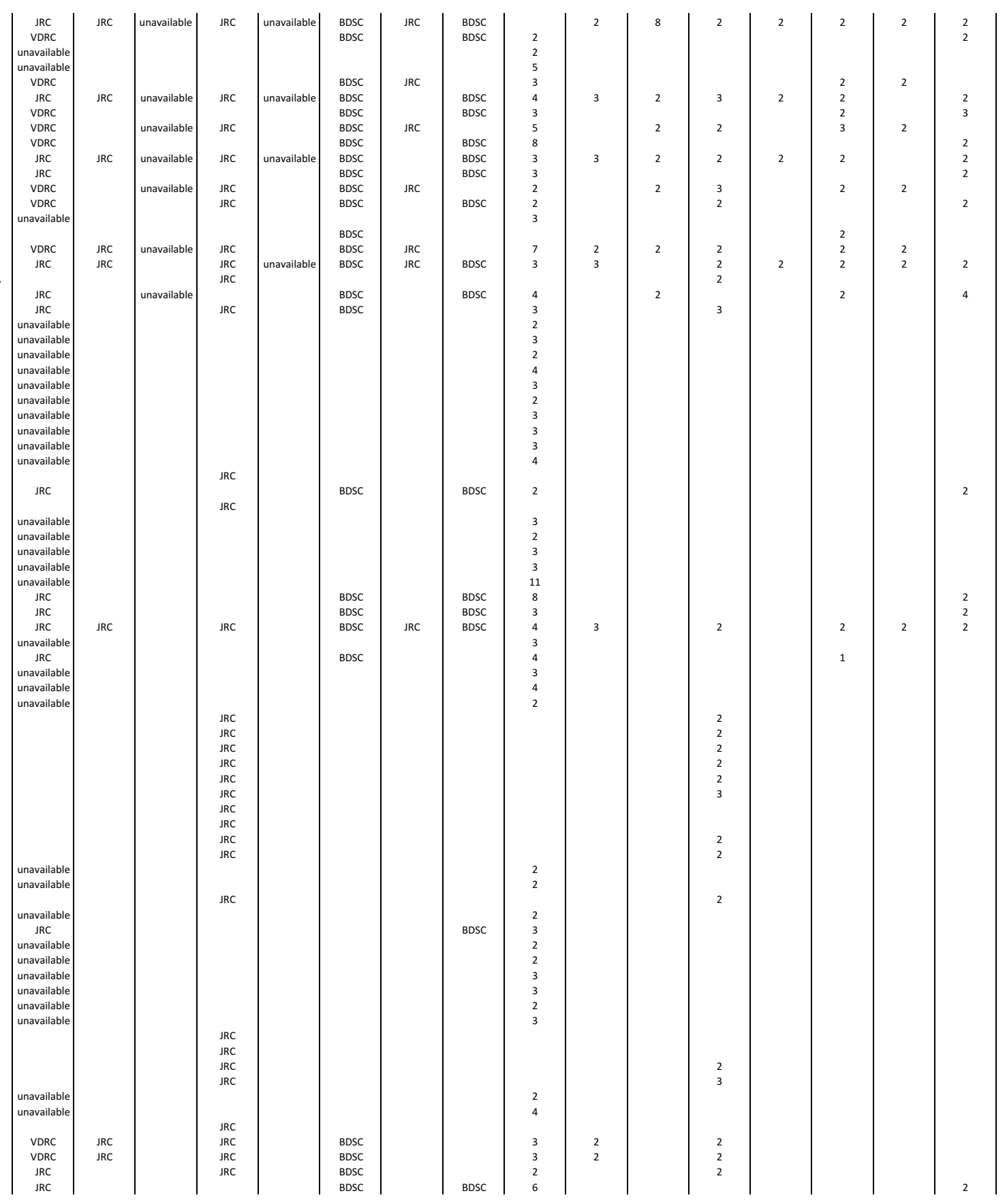


\begin{tabular}{|c|c|c|}
\hline \multicolumn{2}{|c|}{ 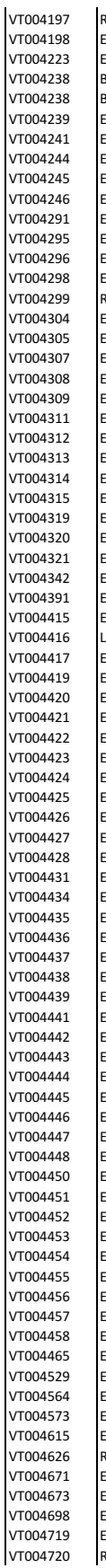 } & 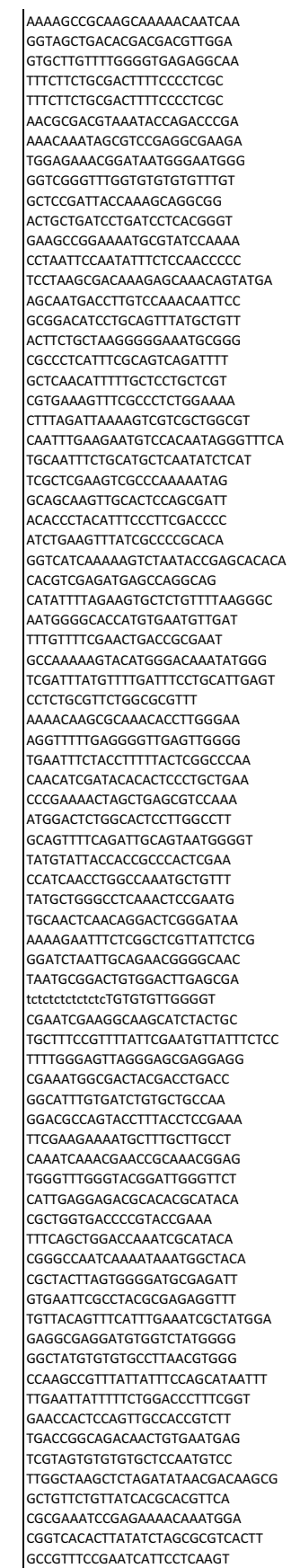 \\
\hline
\end{tabular}

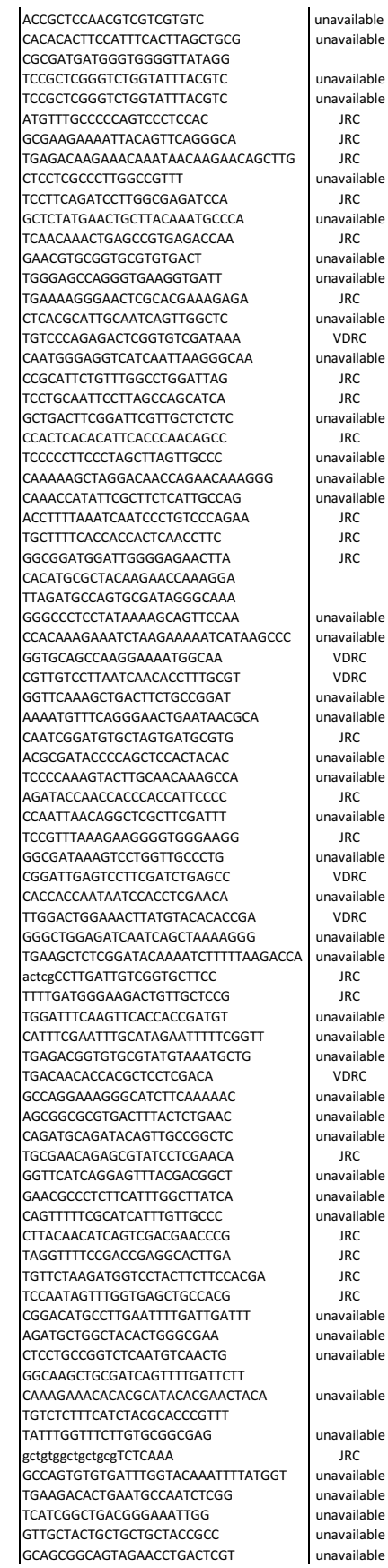
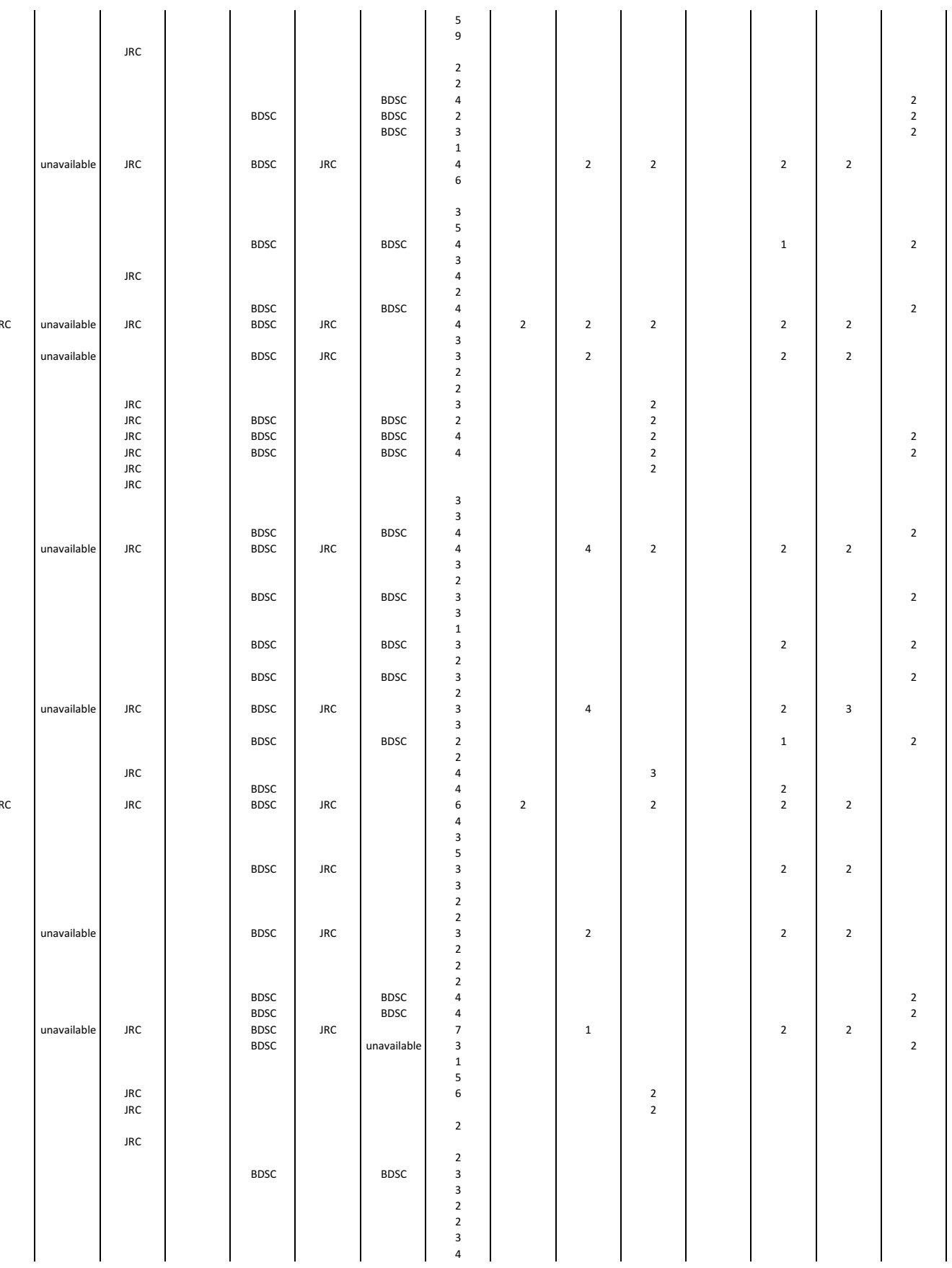


\begin{tabular}{|c|c|c|}
\hline \multicolumn{2}{|l|}{ 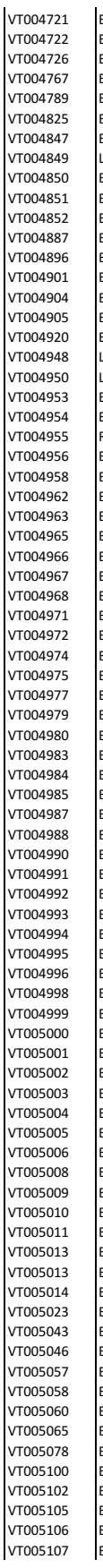 } & 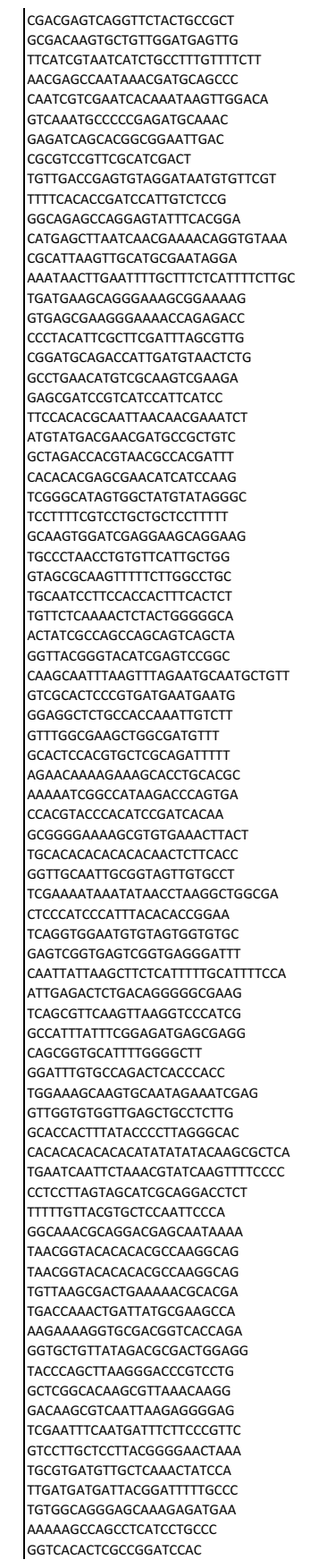 \\
\hline
\end{tabular}

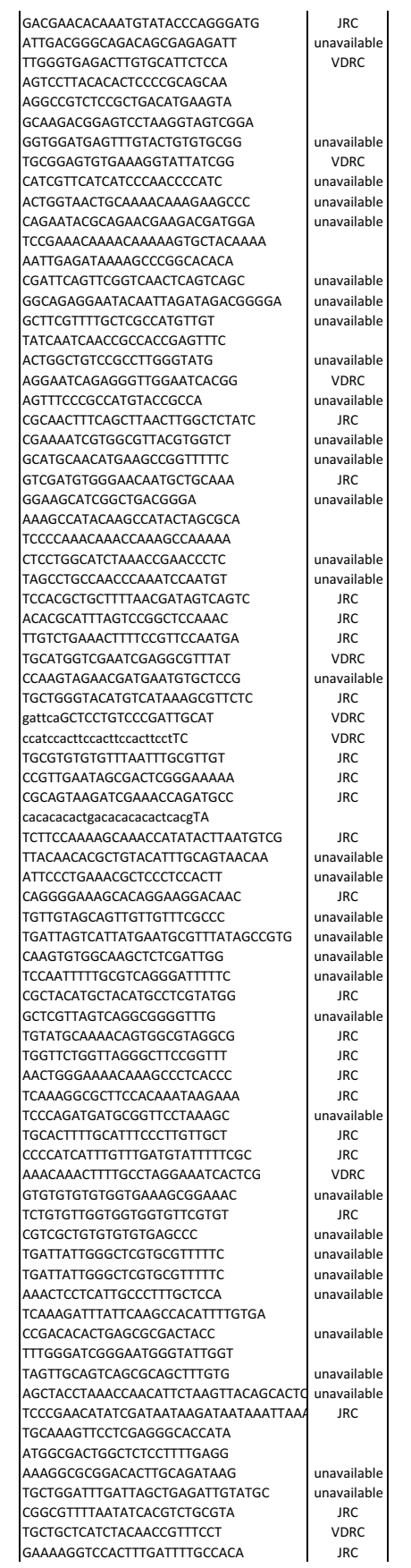
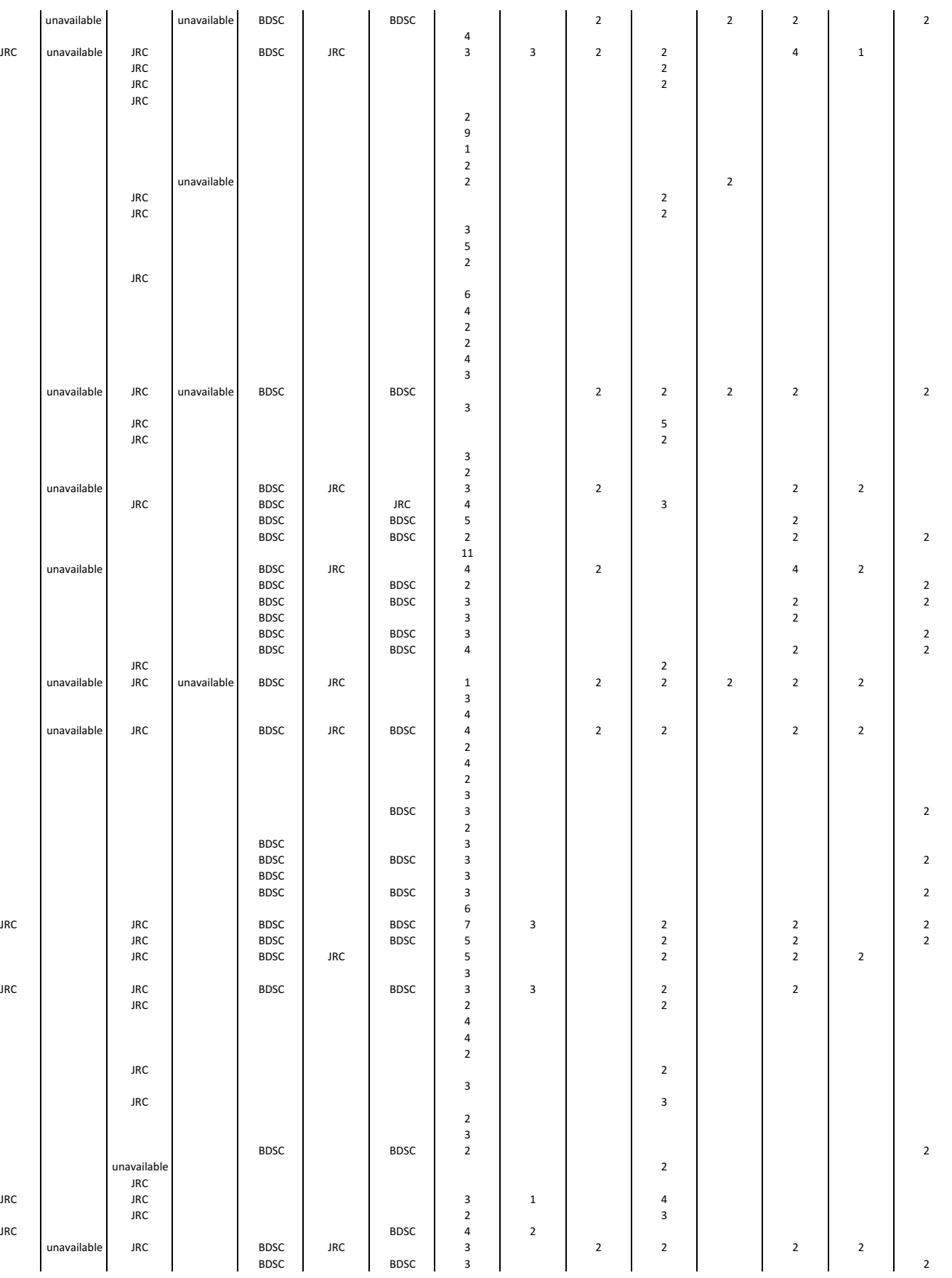


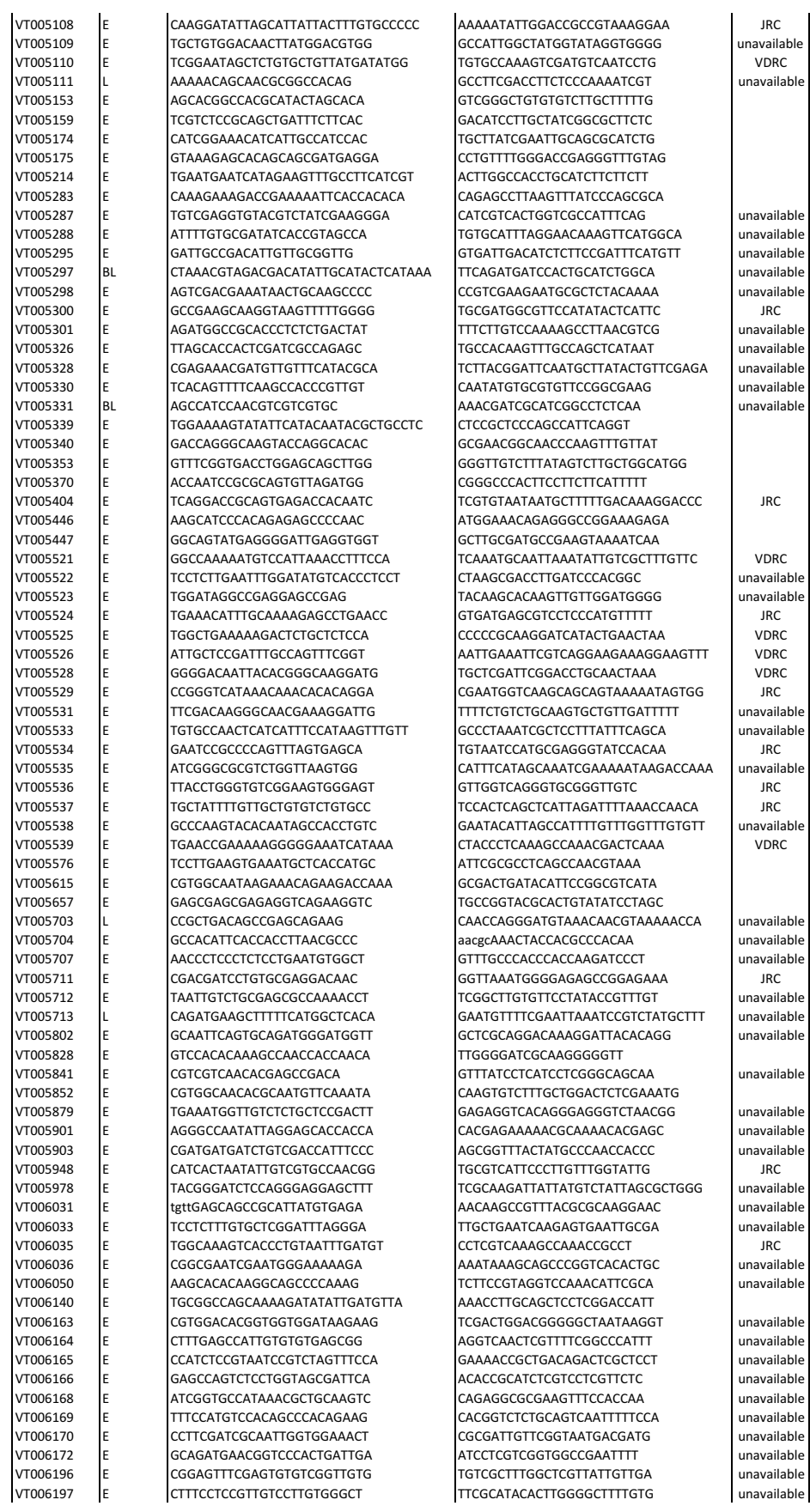
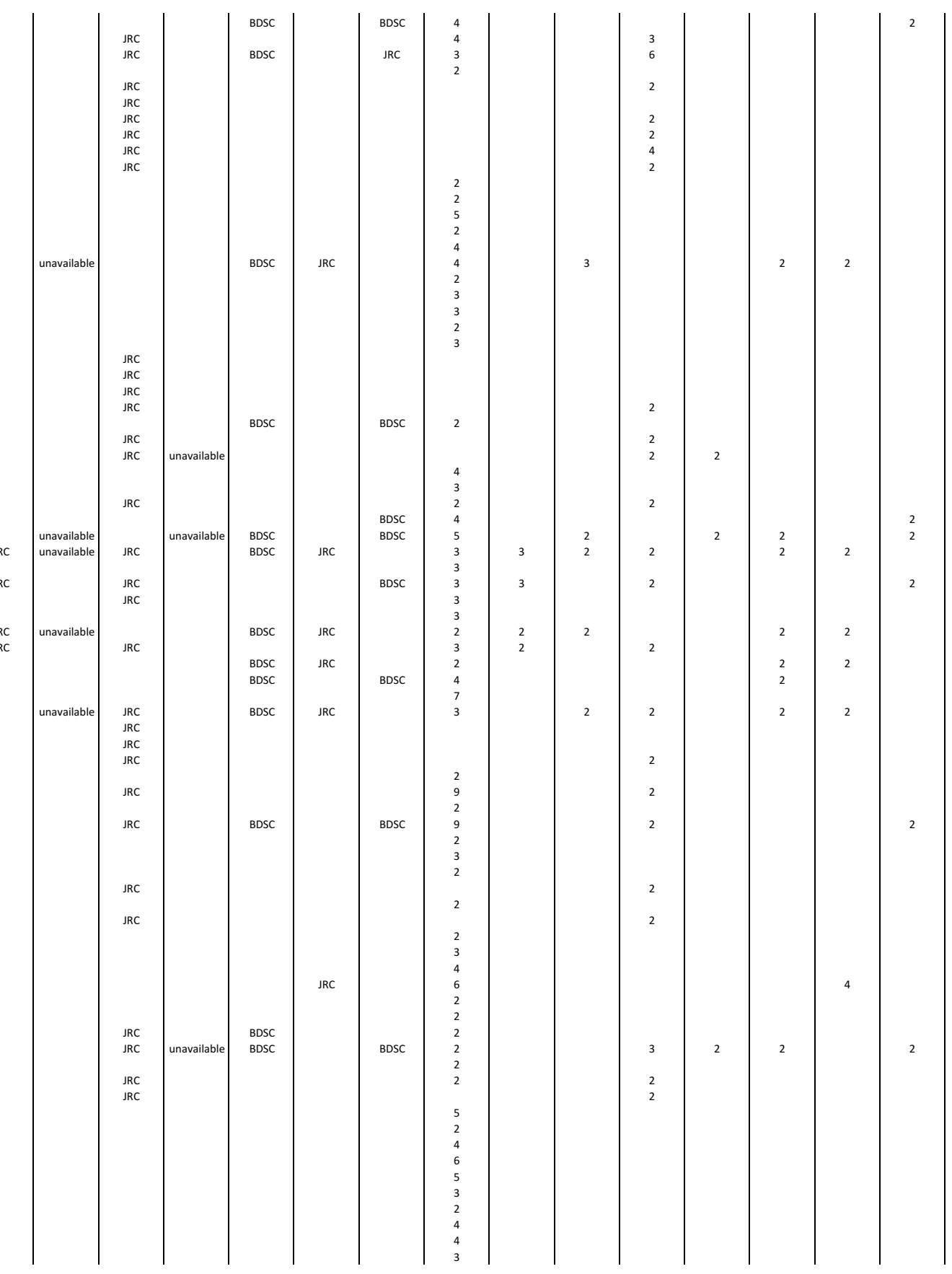


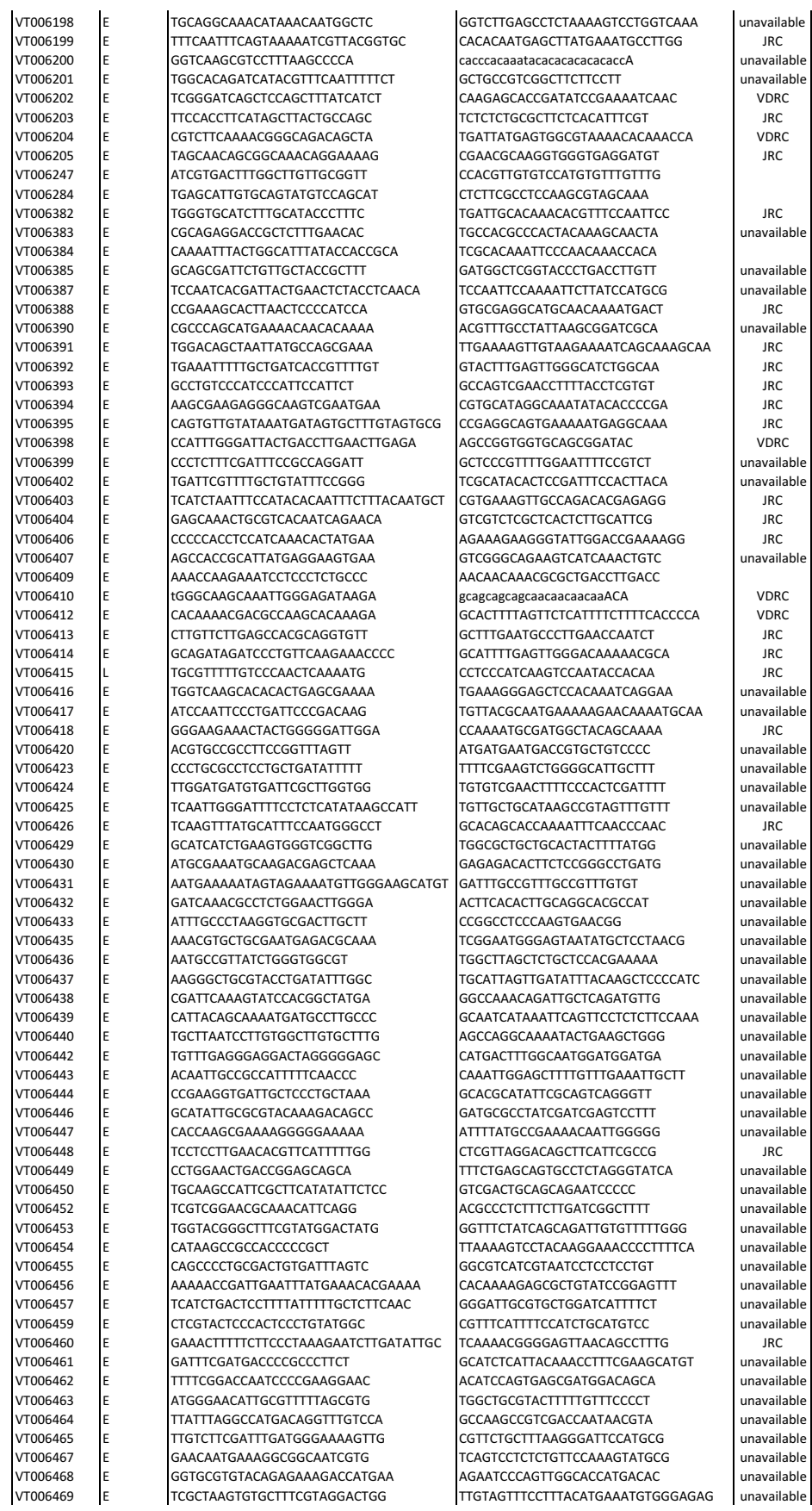
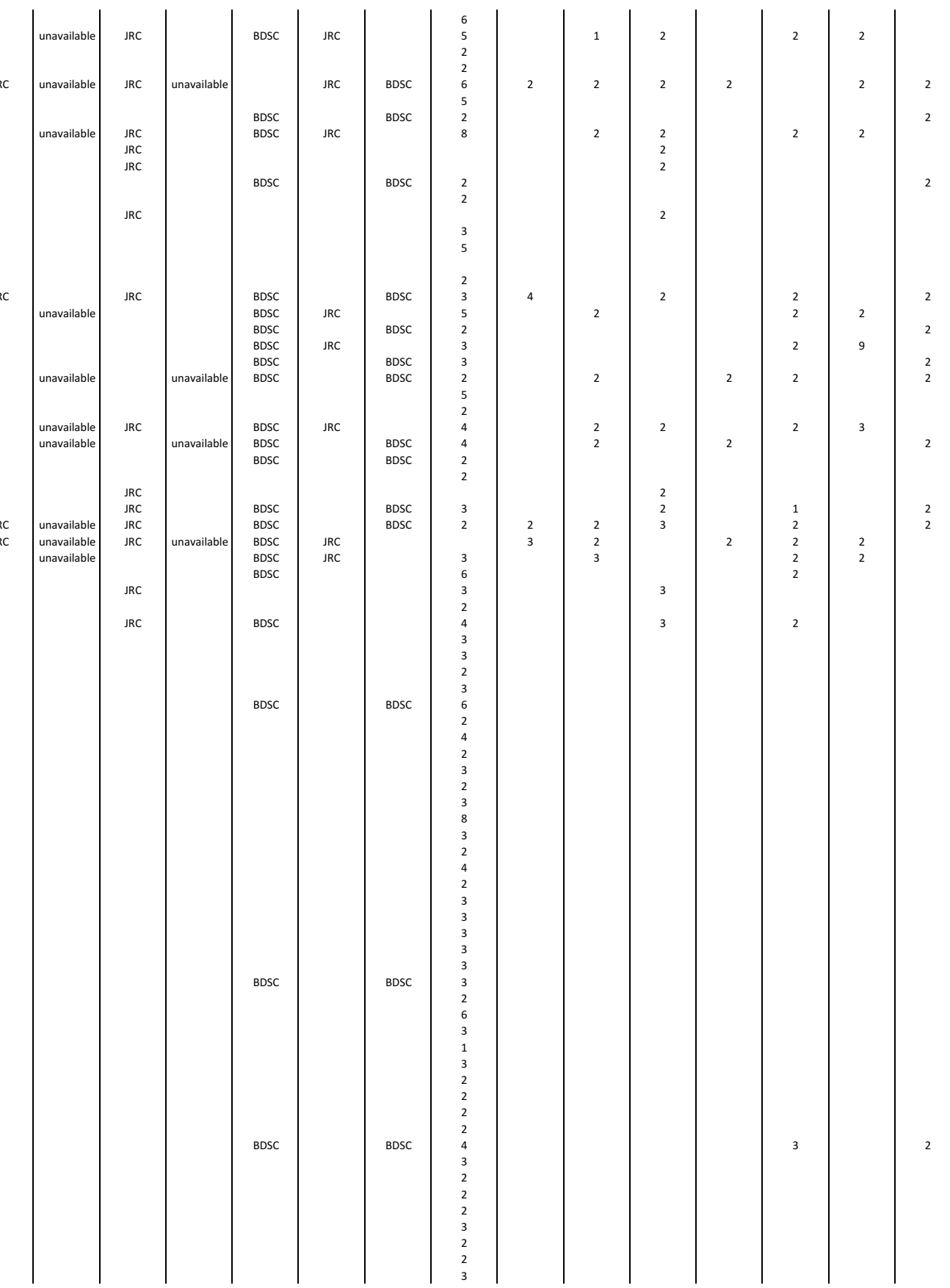


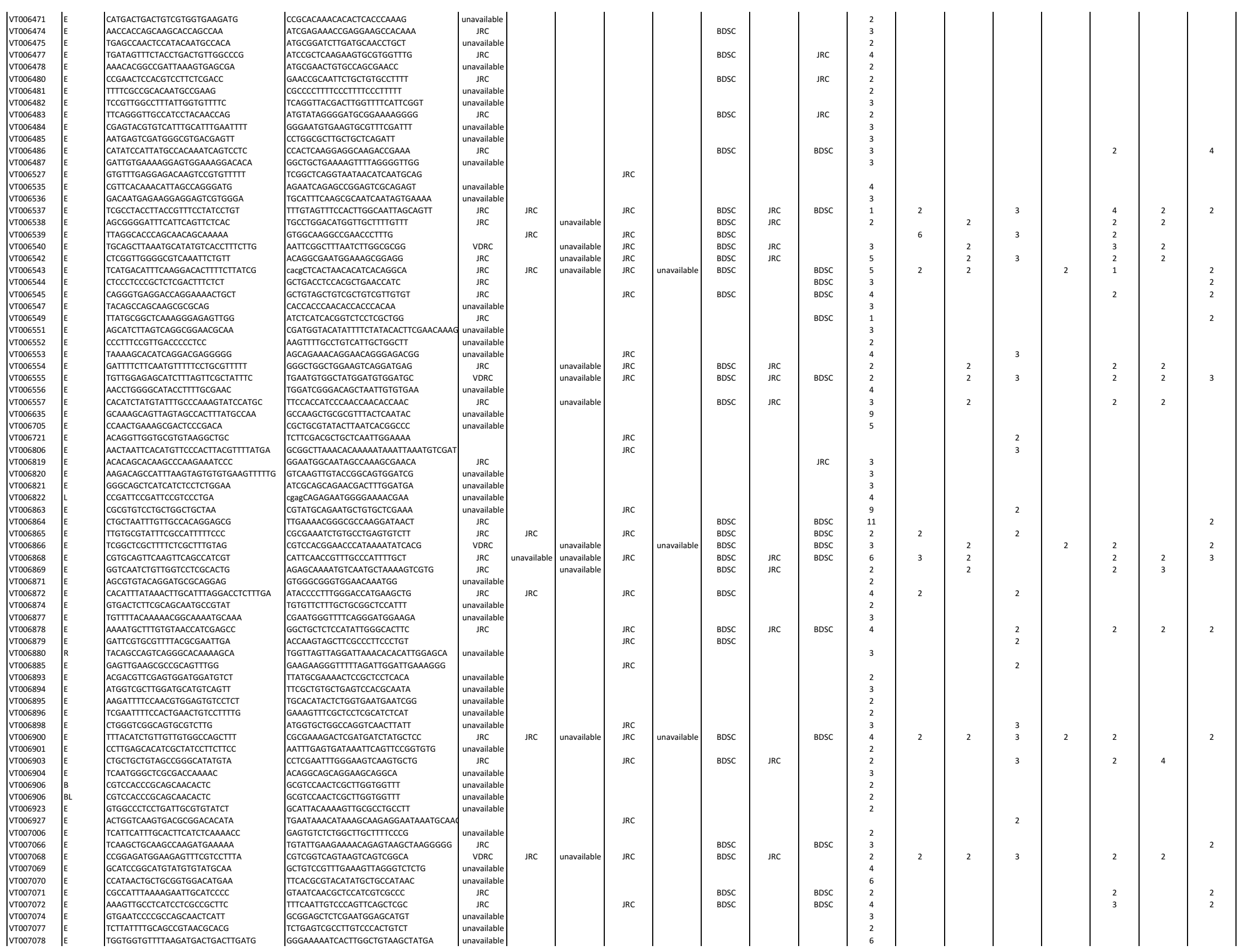




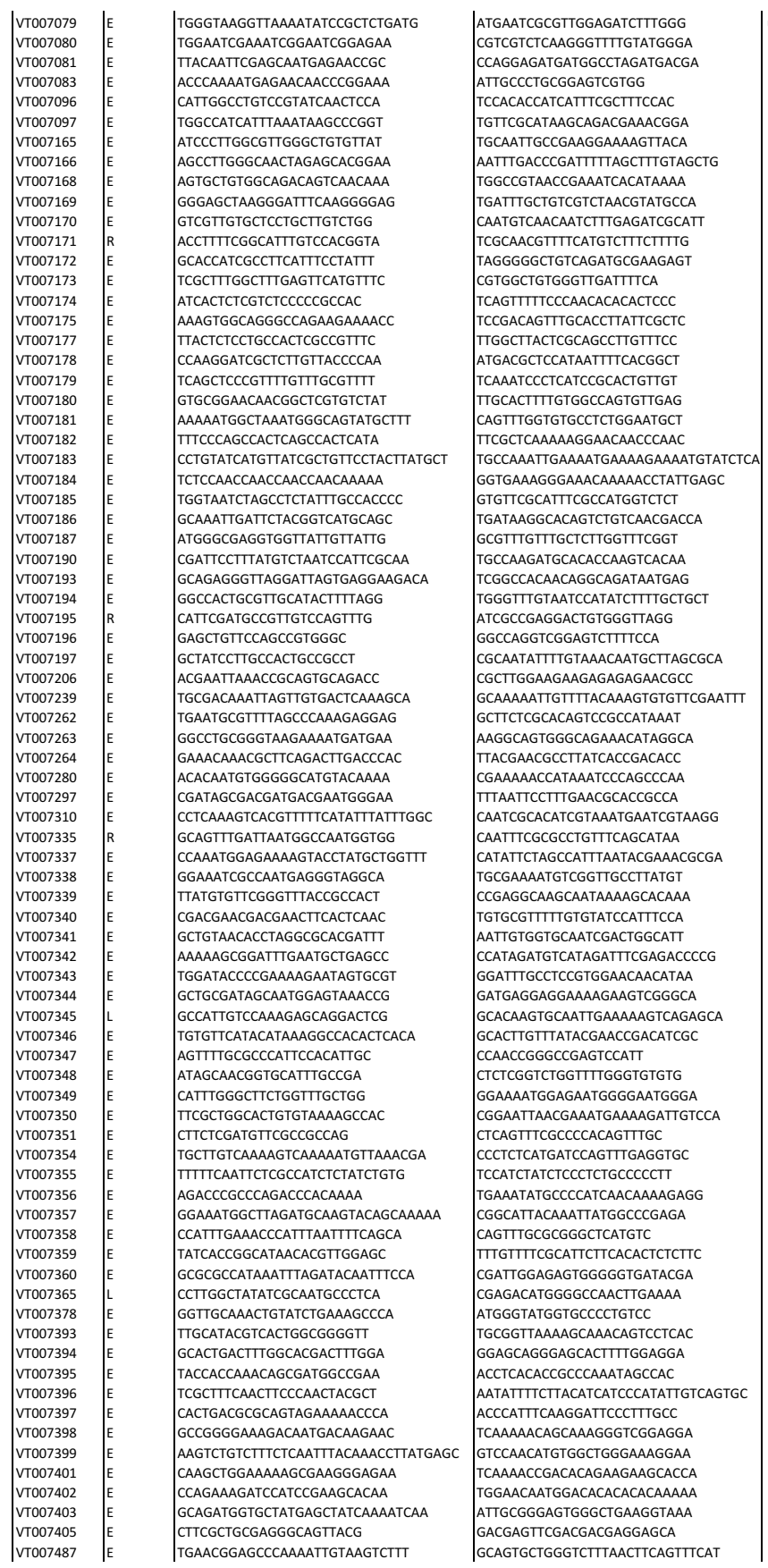

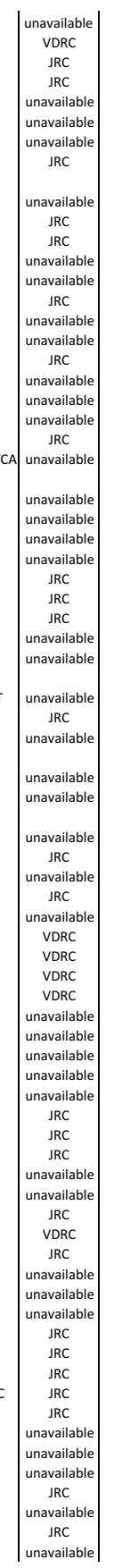
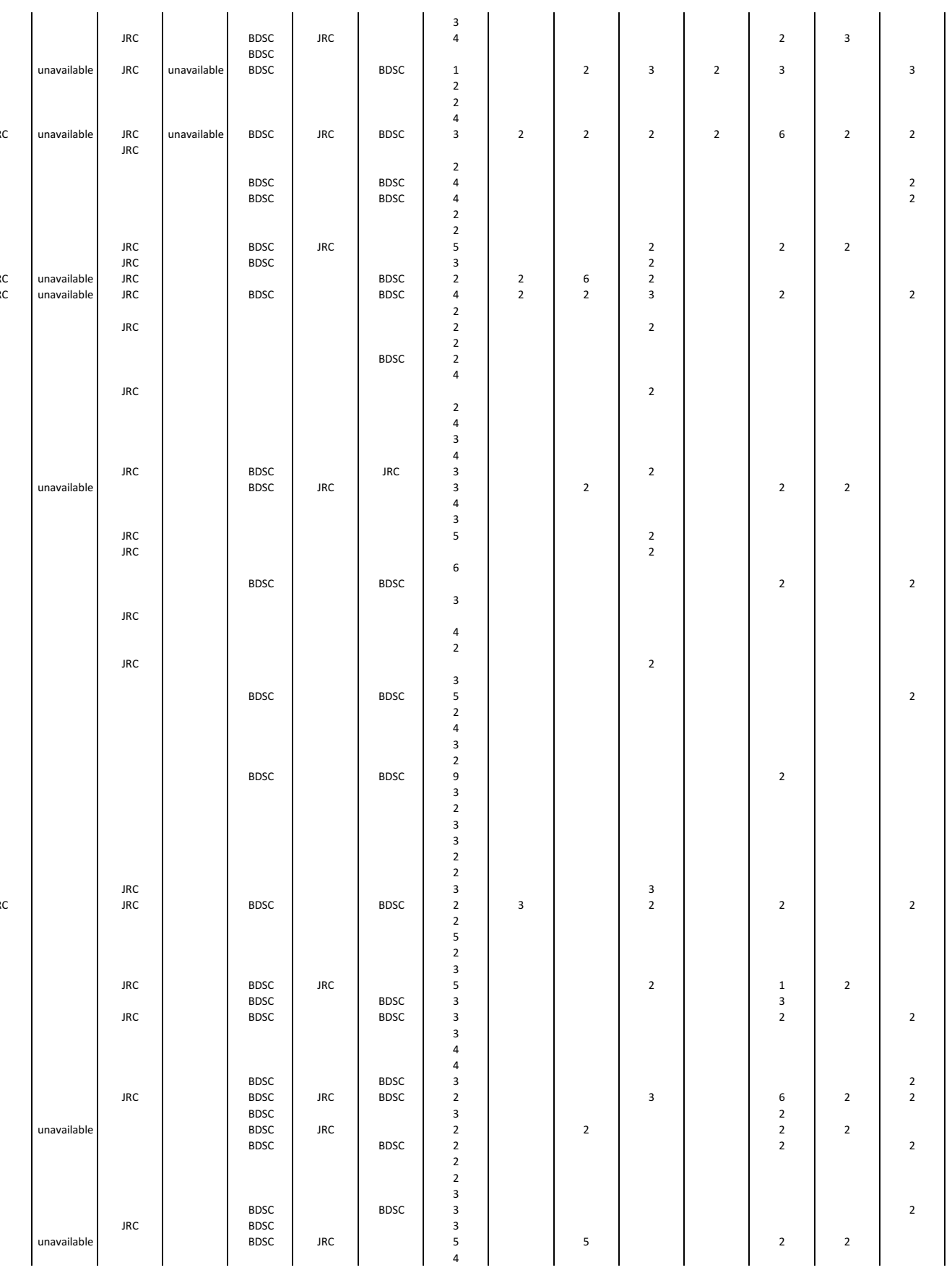


\begin{tabular}{|c|c|c|}
\hline \multicolumn{2}{|l|}{ 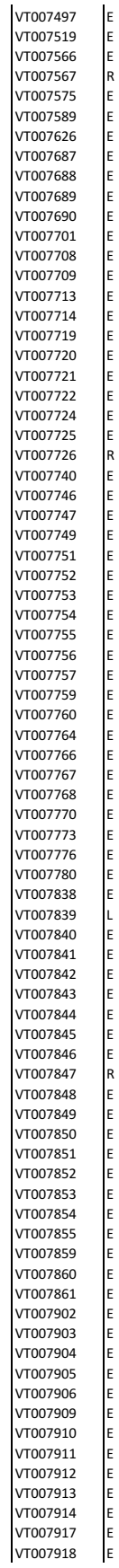 } & 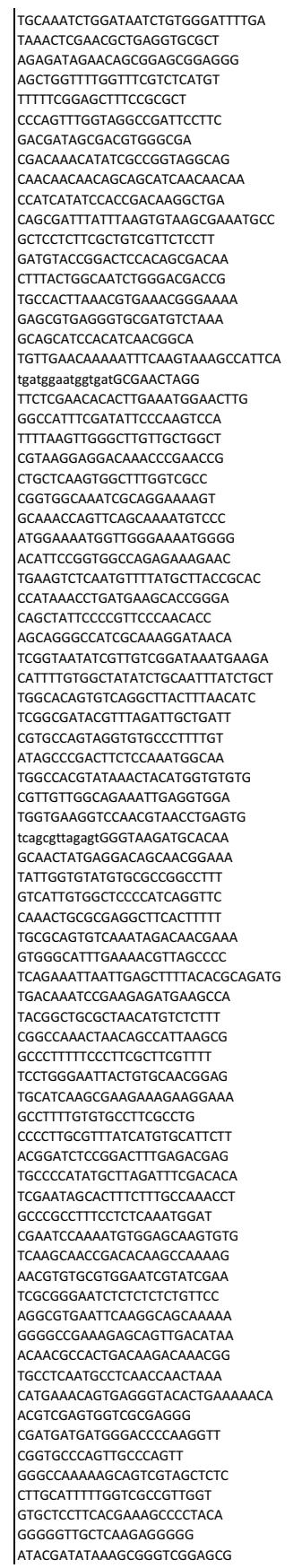 \\
\hline
\end{tabular}

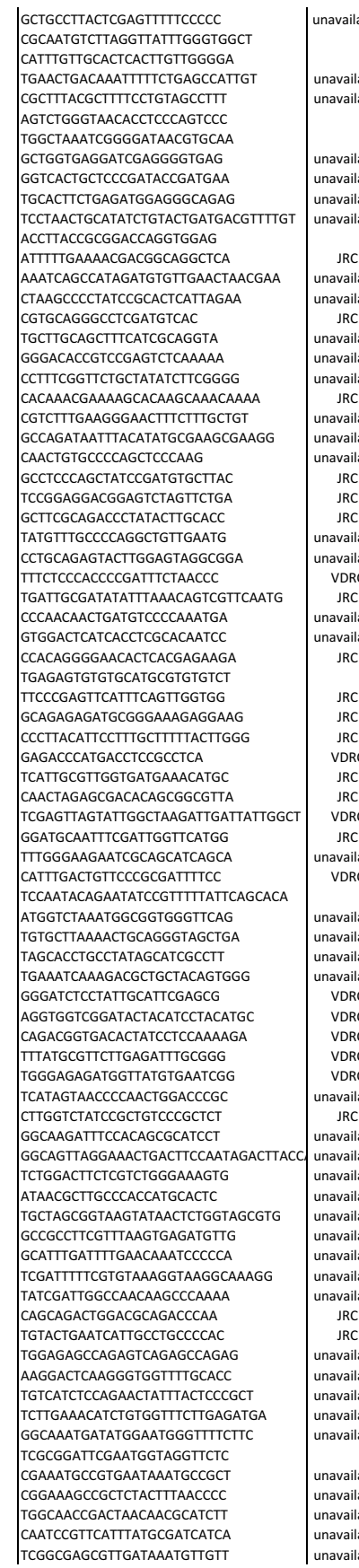
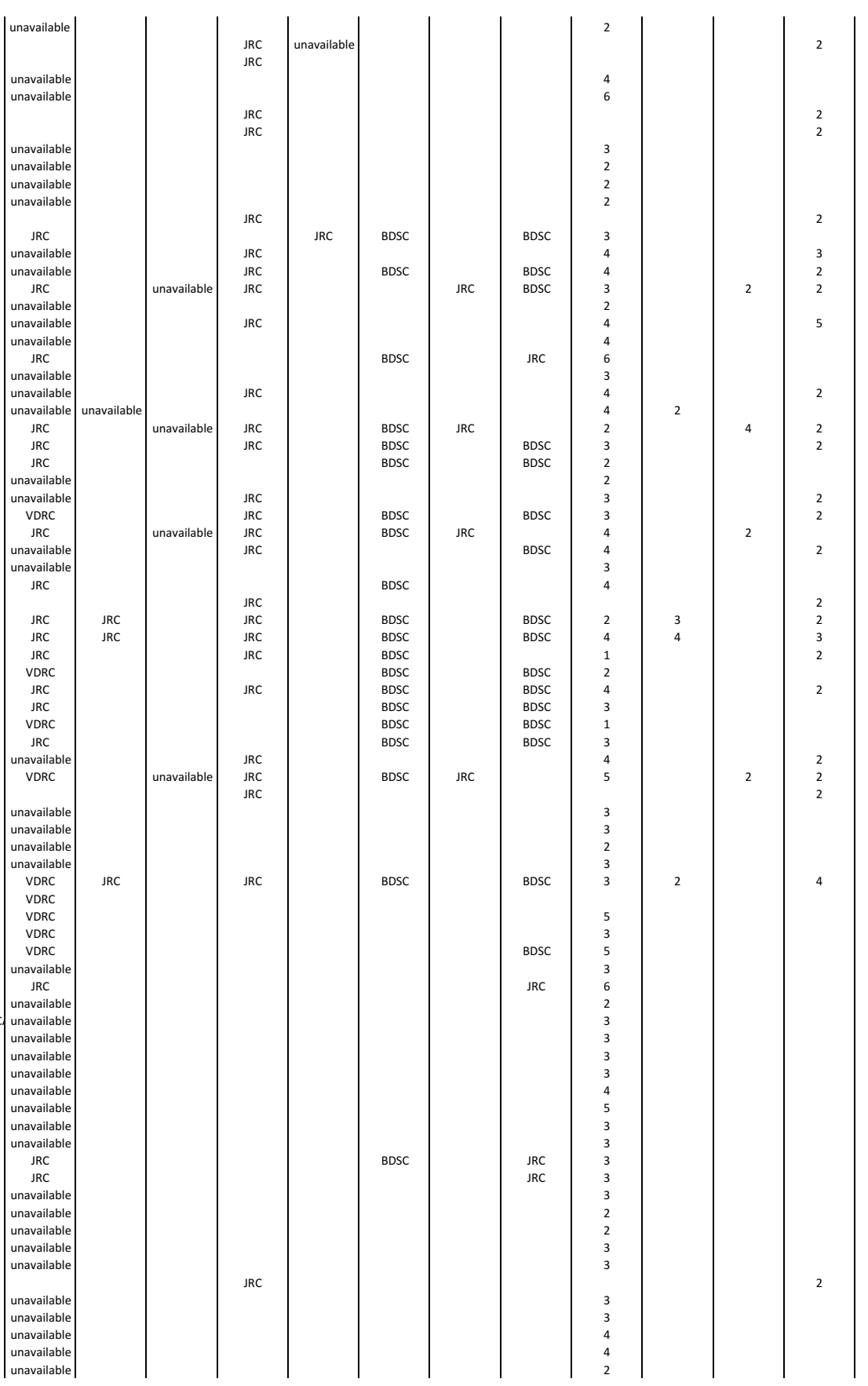


\begin{tabular}{|c|c|c|c|c|}
\hline vTo07920 & $J^{\mathrm{E}}$ & TGAATACGCTTGTGAAAGTATGCCGA & ТGТТАAGATTATTCСТAGСТTAА & vallable \\
\hline vT007922 & E & GATTCTGCAGGTGCTGGGATGAAG & AGGATGTGACTCAGGTAGGACCCCG & unavailable \\
\hline 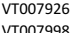 & $E_{B}$ & GGTGGTTCTTGGCCCCAACTACTIT & $\begin{array}{l}\text { CGCTCCAAAAAGGGGCAGTGAATCT } \\
\end{array}$ & unavailable \\
\hline $\begin{array}{l}\text { Vitoong998 } \\
\text { vToo8036 }\end{array}$ & ${ }_{E}^{K}$ & 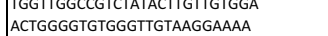 & \begin{tabular}{|l} 
AAGGGGTATTAAAGGGGGGTGGGAA \\
GGCTAACAATAATGAGTGGAATAATTATACC
\end{tabular} & unavailable \\
\hline vT008050 & E & CCTGAGGGAAACTCACACACAGCA & CAGCCCAAAAAGGACGCACAAAAA & \\
\hline vT008055 & E & ATCACTAAGCCAGCTCGGGGACGAT & TGTTTITCATTGTCGCTGTCCGATT & unavailable \\
\hline vT008056 & 每 & GTGGACGTGGAAAACGCCAACAA & TGAAGGCCCGATAACGCGATAGACA & unavailable \\
\hline & BL & GTGGACGTGGAAAACGCCAACAA & TGAAGGCCCGATAACGCGATAGACA & unavailable \\
\hline vT008069 & E & & CGAAATTGCGGAGGTAAAATCGG & unavailable \\
\hline vT008089 & E & СATTGGATTCAATTATTATGTCGGTCTAGGAA & CAGCTGTTCGTGAATATGACTCTGTTGTTAAA & \\
\hline vTo08102 & E & ACCTGAAAAACTCGGCTTGTCCTTG & CCACATGTATCGGGCTGGGGGT & \\
\hline 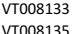 & E & $\begin{array}{l}\text { CGAGCGTATGTAGCTGTGTGGGTTCA } \\
\text { GCACGGAGCACGGCCAGGAA }\end{array}$ & $\begin{array}{l}\text { TGACAAGCGGCAAGAAAACT } \\
\text { TCAGCGTCATATCGC }\end{array}$ & VDRC \\
\hline & E & 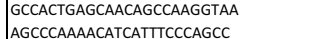 & $\begin{array}{l}\text { TCAAGCCGTCAAATATCAGC } \\
\text { CAATCCCAGCTGCCAAAA }\end{array}$ & VDRC \\
\hline $\begin{array}{l}\text { Vitoo8136 } \\
\text { vToo8142 }\end{array}$ & E & $\mid \begin{array}{l}\text { AACCCAAAACATATATTCCACGGCC } \\
\text { GCGCACTTAGGAGCAGTAGGTGG }\end{array}$ & 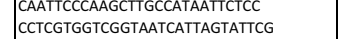 & $\left|\begin{array}{c}\text { unavaliable } \\
\text { vDRC }\end{array}\right|$ \\
\hline vToo8145 & E & ТСGATCTATTTTCGTCTITTAATTGCGTT & AAAGTGTACTTGGGAAGCCGCCTTTA & VDRC \\
\hline vTo08147 & $\mathrm{F}_{\mathrm{F}}$ & TGTCCITIGCCCACAAATATGATTGTTT & CGCATCACCGAAGGACATTGTAGGA & unavailable \\
\hline vT008148 & E & GGCAATGAGAGATAAGAATGTGGAGAAGGTG & GCAAGTTTGTAGGATAAGTGGCTGCGG & unavailable \\
\hline vTo08149 & E & TTAACGTATTCCTTTCCGTCCTTCA & TCAGCACACACAGGCAGCACTTTTC & \\
\hline vTo08150 & E & TCCAAGCTGAAATGAATTGGGCTGA & GCAAATGAGCGAGAATGCGGCTA & VDRC \\
\hline vTo08152 & E & GGGCCAGCGCACTAATGAACTATGAA & CGAAGAAGTGGTAGCGAAAGGAACAC & JRC \\
\hline vT008153 & E & GGAGACATTCCTTCAAGTTTTCGGG & TGGGTAGGTCCATACACTCTTGGGTT & JRC \\
\hline $\begin{array}{l}\text { vTT008154 } \\
\text { vTrosist }\end{array}$ & ${ }_{\mathrm{E}}^{\mathrm{E}}$ & $\mid \begin{array}{l}\mid \text { GCCAATGCACTGGACTGAGCTTTGG } \\
\text { GTGACTGGGCCGTTGTIGTCC }\end{array}$ & $\begin{array}{l}\text { ITGGATGGATCACACATGTAACGAGGAA } \\
\text { GAAAAGCGGAAGAATGGTCC }\end{array}$ & JRC \\
\hline $\begin{array}{l}\text { Vitoosist } \\
\text { vToo8159 }\end{array}$ & ${ }_{\mathrm{E}}^{\mathrm{E}}$ & 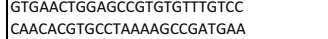 & 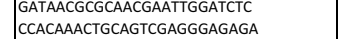 & $\left|\begin{array}{c}\text { unavailable } \\
\text { vDRC }\end{array}\right|$ \\
\hline vT008167 & & GTGAATCCAAAGTCGAGCAGACCCC & TAACGCTCTACGCCTTTTCTCCGGT & JRC \\
\hline vT008168 & & CCCATCTTAATGCCAAATGCCGAAA & TGCAGAACTITTIGATTTACTATCCGAGCG & IC \\
\hline vT008169 & E & GGCAGCAATTTAATGTGAAACAAGGCA & GGCCTCGTACTGCTGCGAAATCAA & unavailable \\
\hline vTo08170 & E & AAATTTTGCAGATTGCGGACCAAC & TCCTTTTCATTTTCACCCGACCACA & VDRC \\
\hline vT008171 & E & CAAGTGCCGTGGTCTGTCTGGAA & ACCGAATCGTCATACTCTTGCACAAAC & unavailable \\
\hline vTo08172 & E & ATGTGGTGGCTCCTTTCCGTTCC & AGCGTATGTGTGGGTGGTCAGCGT & JRC \\
\hline VTo08174 & E & AATCCAGCCCTAGGA & TGCATTCAGCTGTCTACAAAGGGAAA & JRC \\
\hline 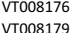 & E & $\mid$ ACCTCGGCACACCACGCATAAAAG & TCAAACAACCCACACATAAAGAGACCGA & JRC \\
\hline & $L^{2}$ & $\mid \begin{array}{l}\text { TAACAGCATCGGCAACGGGTCCT } \\
\text { AGGGTACACCGGACCACAGGA }\end{array}$ & $\begin{array}{l}\text { TGCAATAAATTCACGTGTAGGCCAG } \\
\text { CCAGCACACACATG IAGGAA }\end{array}$ & un \\
\hline 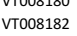 & E & $\begin{array}{l}\text { AAGGGCATCAAACACAGACCACAGGGA } \\
\text { AGGGACACAGGGGGATTGGT }\end{array}$ & $\begin{array}{l}\text { CCAGCCACAACACATGGTAGGAAA } \\
\text { TGAAAATAACGTGCACTIAAGACCGC }\end{array}$ & 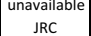 \\
\hline vT008183 & & ACAAAAATTTCTGCCGGGATTCCAA & TGAATGCAATGACAAGGATAACGAAAACA & JRC \\
\hline vT008 & & GAGAAGAAGTTTTGGGACATAAACAAG & TGAAATTGGGGCAGATCCAGCA & JRC \\
\hline vToos187 & & CGGTTGGAAAAGCTGGCTAAATGGA & ATTTTTACCTTCTGCTGCTCGACGG & JRC \\
\hline vT008188 & & GAAAGGTTGTTTTGTCTTTAATTTTGGGG & TGGAATTTTAATTTGCGTTCTCCACTTCCT & VDRC \\
\hline VTo08189 & E & TGGAAGTATGGGCCTTGCCAAAAAG & AAGTGAGGAGACACAA & unavailable \\
\hline VTo08191 & E & CAATGTTCGGATTGTGTGTG & CAGTCCC & VDRC \\
\hline 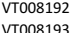 & E & GACTCGCACAGTGGGTCAGC & $\begin{array}{l}\text { GGCTGGTGGTTGGGTTGA } \\
\text {-GCTGGAGCAAGGACC }\end{array}$ & unavailable \\
\hline $\begin{array}{l}\text { vitoo81933 } \\
\text { vToos } 194\end{array}$ & ${ }_{\mathrm{E}}^{\mathrm{E}}$ & $\begin{array}{l}\text { CTGGTITCCTGGGITTCCCCA } \\
\text { GCAAAAGGAAATCAACAGCCCCA }\end{array}$ & $\begin{array}{l}\text { TTCCAAGAGGCAAACGGACGITICA } \\
\text { TCAACATTGGTCAAAATACCCCA }\end{array}$ & $\begin{array}{c}\text { unavalable } \\
\text { JRC }\end{array}$ \\
\hline vTo08197 & E & CCGACTTTAGCAATAGACAAAAGCTG & GACGAAAGCAAGGGGAAAAACTTCAAC & unavailable \\
\hline vTo08200 & & GTCCTCCTGCATCCCCCATCCTT & GCTCCACCGCCCCCACA & JRC \\
\hline vT008208 & E & TGCAAATCCATTGTGTTATTGGCATAG & TGCCTTCAAGACTGTGAGCATAGCATTTT & JRC \\
\hline vTo08217 & & СGCTTCA & GAGCCATTCAACGCCGA & $\mathrm{IRC}$ \\
\hline vTo08218 & & GGAACTTGTTGGATTTCTTATGGCGG & AGGTAA & unavailable \\
\hline vTo08223 & & GAAGCGAA & AG & unavailable \\
\hline vTo008253 & E & TCGACAA & СTCTCGCTCATCAGGTGGTCGTT & unavailable \\
\hline vT008256 & & TAGACGITATTTTGGAGGGGG & CGGGGTGTGGAGTGTTGGC & \\
\hline 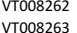 & & $\begin{array}{l}\text { CACTCACCATGGACAGCGAAAC } \\
\text { GCGGCAACTITTCTIIGC }\end{array}$ & $\mid \begin{array}{l}\text { GGGGCAGTTGGGGGAAC } \\
\text { AAGACTCCATCTGT }\end{array}$ & unavailable \\
\hline $\begin{array}{l}\text { VTo82683 } \\
\text { vToos267 }\end{array}$ & E & $\mid \begin{array}{l}\text { GGGGTAAACTICTCCTITGCACT } \\
\text { GATTGCAGCGGGATGGGACCT }\end{array}$ & $\mid \begin{array}{l}\text { AAAGACACCCAGACCTCGTGGGGAA } \\
\text { TCTGACTGGGACGTGCTGAGGG }\end{array}$ & $\begin{array}{l}\text { unavalable } \\
\text { vDRC }\end{array}$ \\
\hline vT008268 & & TGTTCC & CATCACGAAGAAGGCACCCA & unave \\
\hline vT008270 & & CAATCA. & GCCACGCCCATACATTCCTA & unavailable \\
\hline vTo08271 & & & CAAAAC & unavailable \\
\hline vT008273 & E & ICACGGTAACGCTGGCT & CGAATA & \\
\hline vT008277 & $E_{F}$ & CTTGGCCTCCC & CATGGCAGGGGTTTGCAGAA & JRC \\
\hline VTo08278 & & CTCT & AAAA & \\
\hline 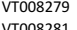 & E & TGGGTCTCCAACTTCTTCATTCGCT & AACGGGGTGTGGC & \\
\hline 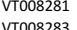 & $\mathrm{E}_{\mathrm{E}}^{\mathrm{E}}$ & ССAAACAAACGAATCAGACGAACCAA & AACACTCGTTGTCGGGCTGTAAGGG & t \\
\hline 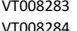 & E & CGACTITGGTGGTIGGCAGGTTC & GCCGITCCGTTCCGCTCACTT & unavi \\
\hline & & $\begin{array}{l}\text { TAACTTCAGGGAAA } \\
\text { TGACAATCCGCAG }\end{array}$ & $\begin{array}{l}\text { AGGGAGGGGGGAAAGGAGAAAAATCG } \\
\text { GGAAGCGGCAGGGAAA }\end{array}$ & $\begin{array}{l}\text { unavailable } \\
\text { unavaiablable }\end{array}$ \\
\hline & E & $\begin{array}{ll}\text { CAGGCAG } \\
\text { CATCACA }\end{array}$ & GAGGGGAAAA & $\begin{array}{c}\text { unavailable } \\
\text { vDRC }\end{array}$ \\
\hline 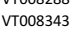 & E & CACATCGGGAATTAAGTIGTGTAATCA & ACTGGTATTCAGCGAGAAA & unavailable \\
\hline vT008344 & E & стССGGGCTTCTCCAGGATGTTC & СТAAACGCTTTCTGC & unave \\
\hline vTo08345 & E & GGAGATAACCCCAGAAGAGCTCAGGAA & ATCGGGCGGAGGG & unavailable \\
\hline vT0083347 & $E_{-}$ & TAG & сGCT & unavailable \\
\hline vT008350 & E & СTTTGTTGCTGCCTCCGCTGCTT & TATTTGTCCGCCTCGCCGTCATAC & \\
\hline To00356 & $\mathrm{L}$ & ACCACCAACATCCTAACCCAAGCCC & ACTTCATTCGATTCCGCTGAACTGC & \\
\hline 8359 & & $\mid \begin{array}{l}\text { GGGGGCGTIGCCCTIATAACACT } \\
\text { GTACACGACAGGGCAGGGCA }\end{array}$ & $\mid \begin{array}{l}\text { AAGGGCCCTTGGGACCCCACTGGTTT } \\
\text { AGACAAAACCTTCGCGTTCTGG }\end{array}$ & $\begin{array}{l}\text { unavailable } \\
\text { unavailable }\end{array}$ \\
\hline & BR & |GTACACGGACAGGGCAGGGCA & AGACAAAAACCTTTCGCGTTGCTGG & | unavailable \\
\hline
\end{tabular}
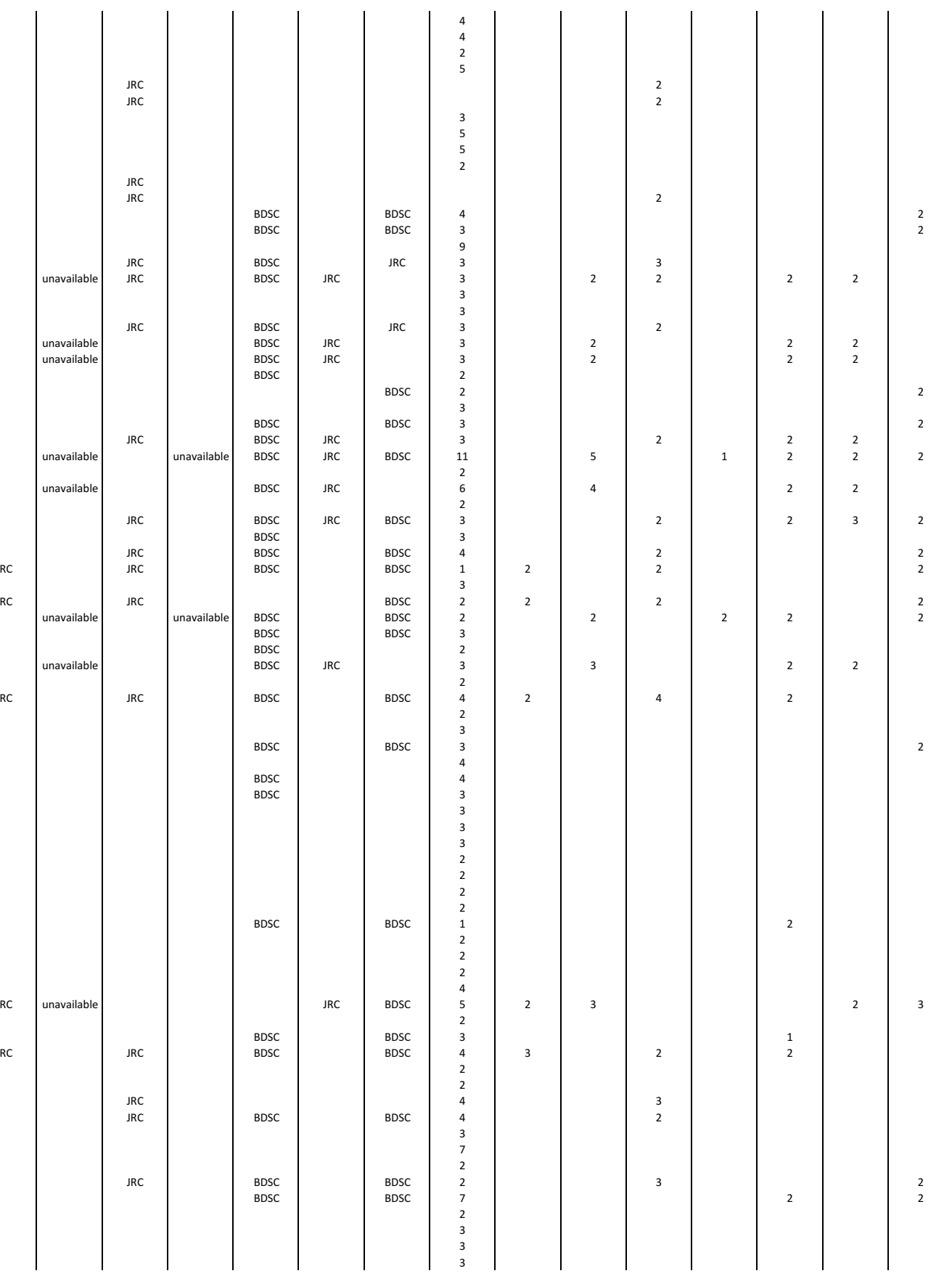


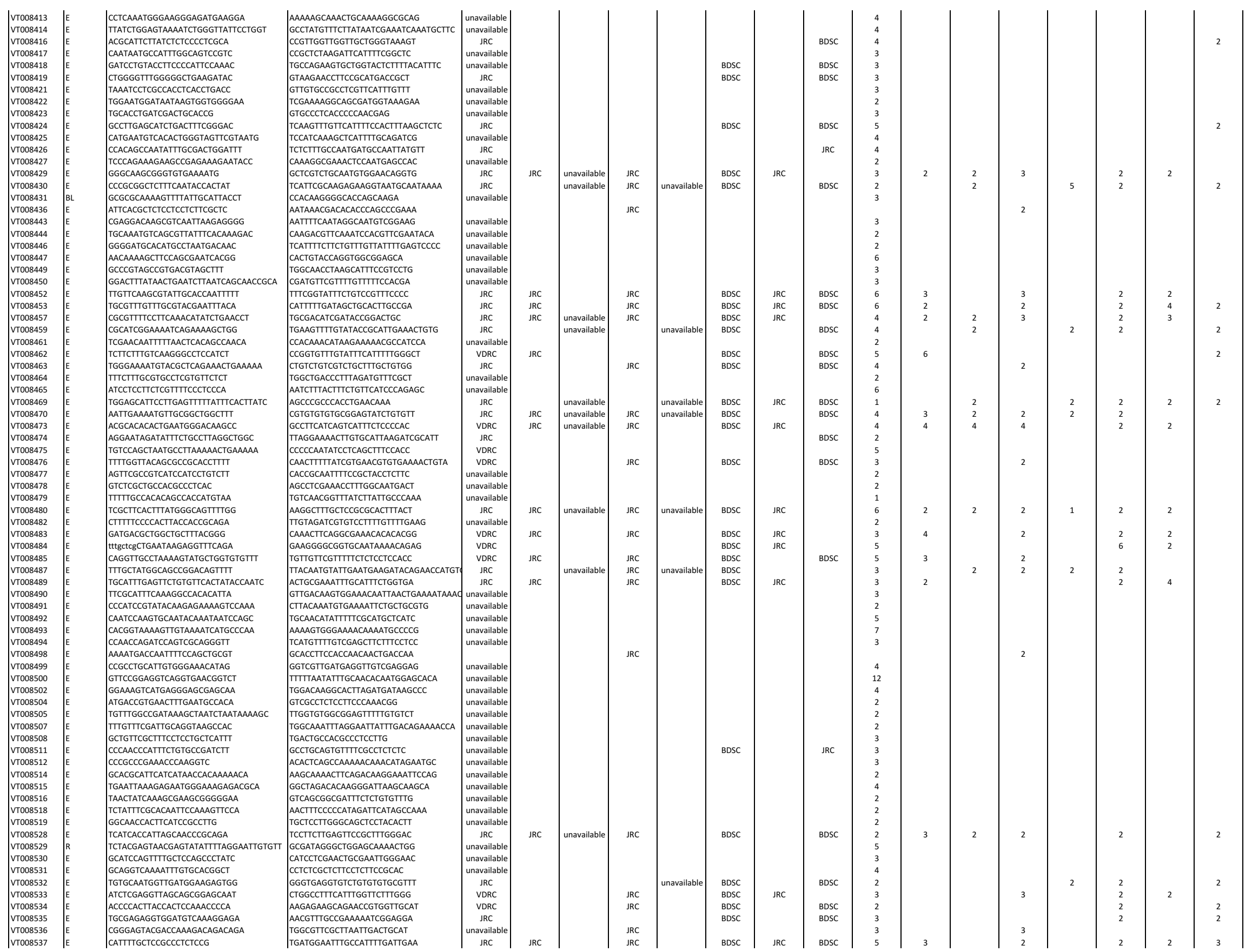




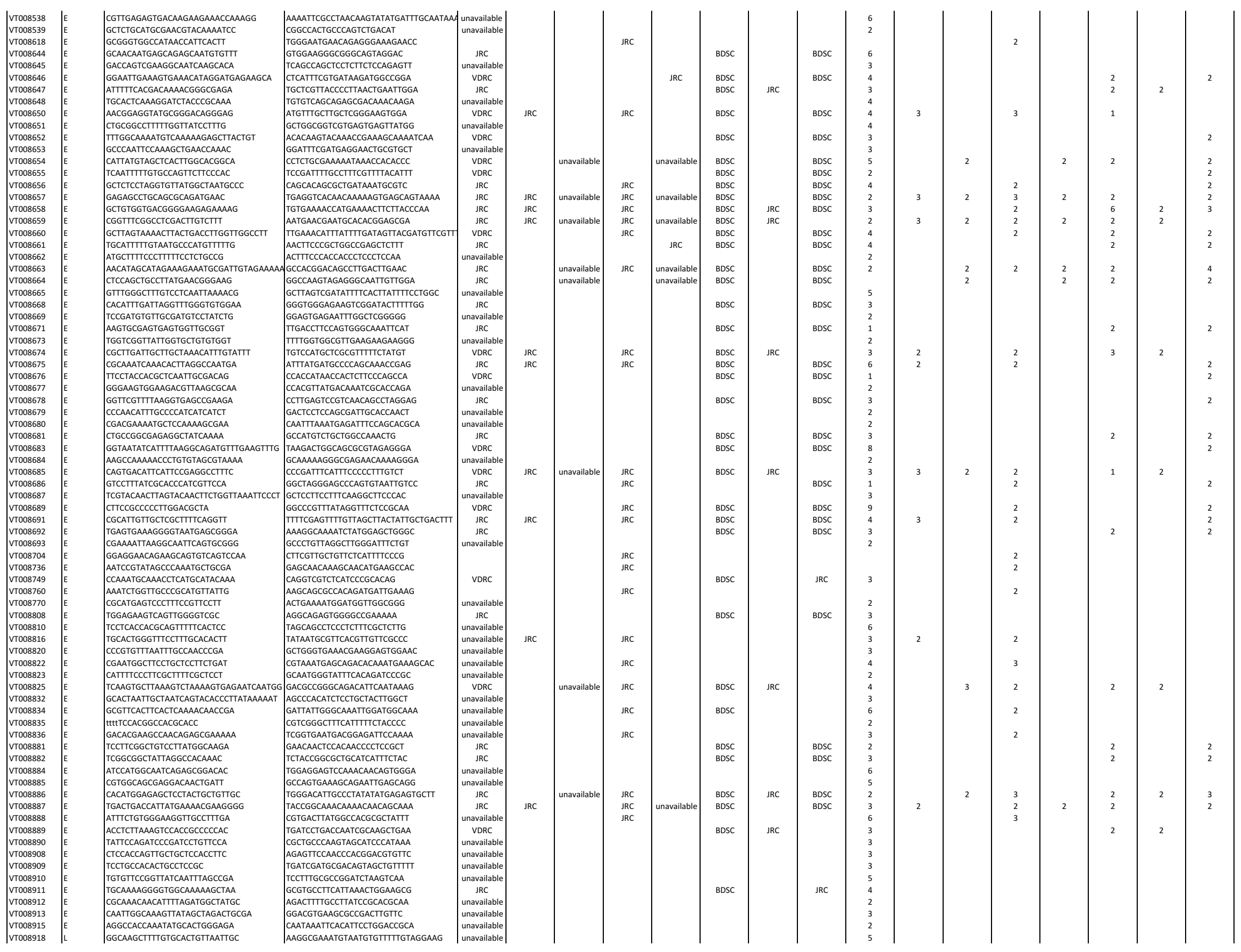




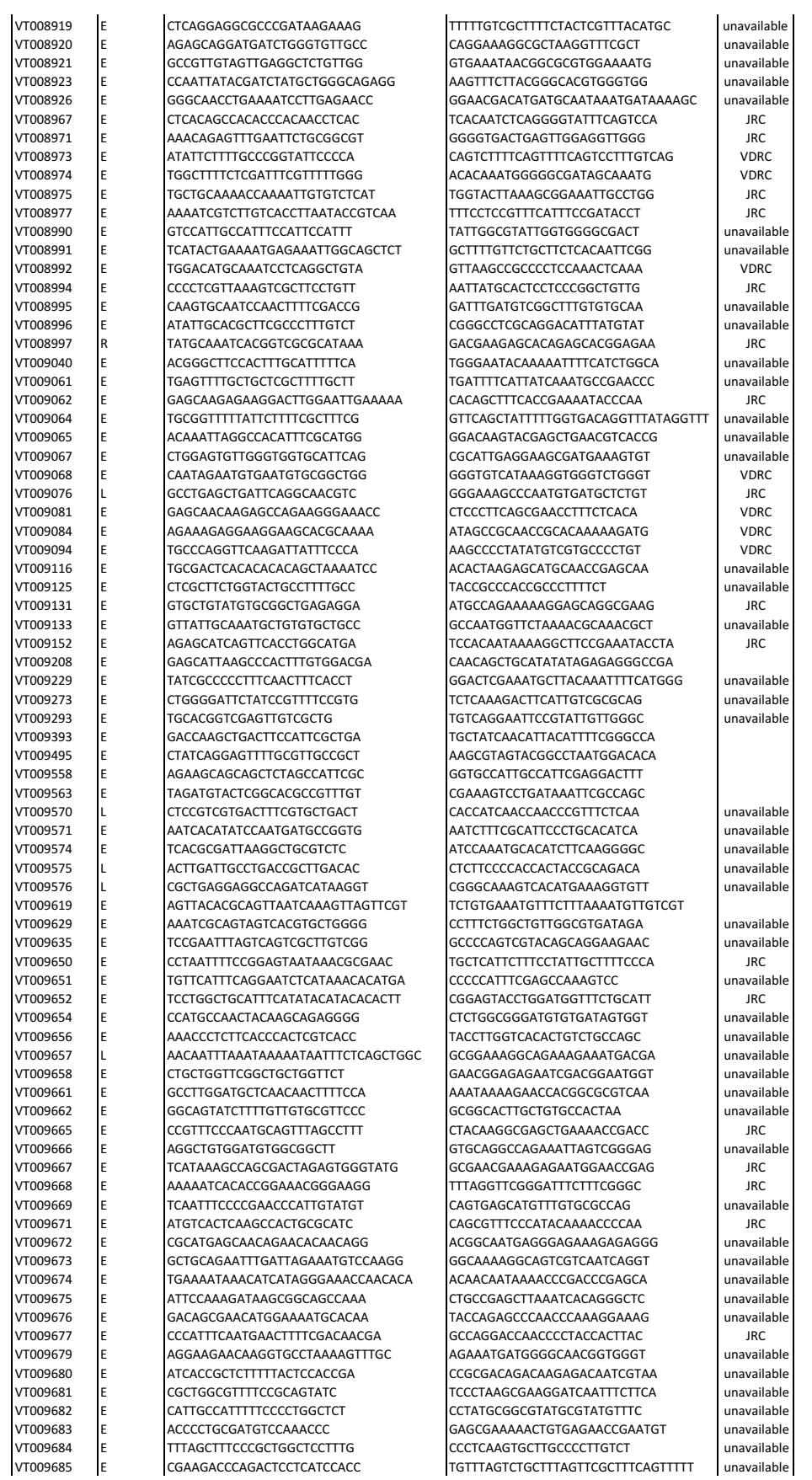
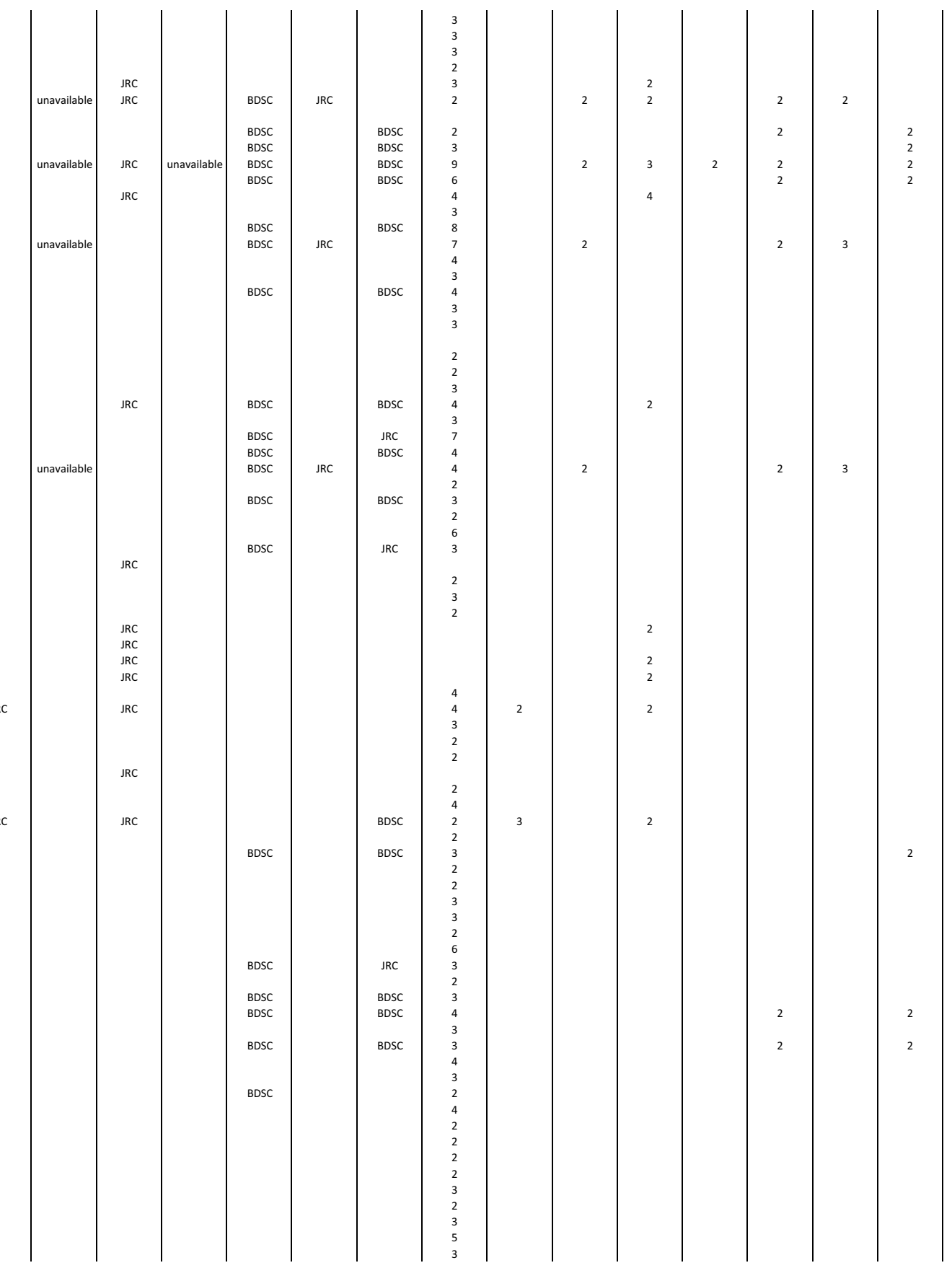
AaAgcctGCAAAACACAATCGGTCC G CAAGCTCGTCTCCTTCTGGTITCCG AGCAAGCCAAGACGACGGGAAG

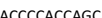
政 CCAATGGACCACCTGA GGGAAGTAGGCATGAAACTTGG ATTGCTGTGCGCTTGGCGTTCTC GCAAGGTGAACTCCCGACC GACGGACGAATTTGTTGTTTTGCCC GCCACACACAGTCCACAGTCCACAC GTAGTGAAATCTCTGGCGTGGGT CCTTICCAACCACCTCTTCGCTTCC GATCTCTTGGTGTGGAGGGTGAA
TCGAGCAAAAGGCTACAAATCACAC ICGATTTCTTCCTTCGAGCCACC CCACCGGAAGTGTGAGGAACCTGA
CCCTAAGTTACCGCCTCCTTGACGC GGAAAAATGGGCGGAAAATGGAA
CGGAAGGAGCAGCAGACAGAACA ICCCAGAGCCAAAAACACACACACA
CGGCTGAAACTCCTTGGACACATC TGGAGGACTTAGGGATGAGAGCGTGA TTTGGCAGTGCCCTTTGCCCTTTT CGGACCCGAGACTCAAGCTCAAGAT AAGACATGAAAAATGTCGCCGCAAG CGGGGTGGACAGGAAGAGAATAGGA AGATGGGACAGGAGGTCGAAGAGGA ATTAGGTGTGAGCCGAGTGGGGATG GGCTCGATGAGTGGCTITITCTCT AACCCTGAGGGTAAATGCAATAAGCTGT TAAGACCCAAATCGTACACAGGGCA ATATGAAGGAAGGCGAATGGCCACA CAACAGCCCTTCCATAATTTGTAGCC GGCTCCAAAGCAGGAAACTGTATCTATT ICCAGATGAACGACCTCCCATGCTA TCTGAAGGACCTGGGCTTGGAACAC CCACTCCCCTCAGCCCATTGAAC TGGGAGTCAAGTTTACCTTCATAAATACAG ICTGACGAATAGTGGGTGGTGCCC
TGCCTAACCACCGGATACCCACTT TAACCCGATGAACCGATGGCTGG CTTCCGTCGATCTGGCACACGCT ACGGATATGGGGATCTGTGCCATTT TCTCTITTCTCGCTTCGCCATCTC CCCCCACCTTCCATTCTCACTGTC AAGTGCCATTTGGCGAGTGAGTGAG AATGGTTTTCAGGCCGTATCTCCG
GCTCTAAAATGACTCCAGACTCTITCG GACTAAAAATGAACTCCAGACTTC
ACCACAATTCTTGCACCACACGCA CCAGCAGAGGGGTAGGGGTAGAGAA GGGGGAGACCGCAAAGCATTTTAA TGATTTGAGGAATGACGCACTTTGG CAATAATATGGTGCTGCGGTGCGG GGCCAATTCAATATCAGGGATTATGCAG GCTTCGAACGCAACATCGTTCATAA AAAATGGGAGTTGGGGCCTTGTC CCAGAATACTGGGGCTTGGGGAAAATAA GGAATTGGCTTTACGGCTGGCT СCCTGGTTATTTACCACCCTGCTCC AAATGTGTCGCAAGTGGCAAGCAAG ITAGGCGGCTGTCGAGCGCATTAG GGCGACTTACACACTGCCAAATGAC
GTGCCGATCCCTTGGGTCAT
GgCAAACAGACGCACACTCGAAAAC

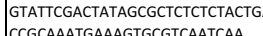
GGGATCCTATCAAACTCCTACCCACA GGCCTAACCTATTATCGCTCACGCAA AGGCATCACCCACCAGCTACCACTC

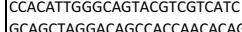
ATCTGGACTGCGTTGCTGGTGC CGCACAGCAATCAGCACCGAAATA TCCAGTAATGGAAAGAAACACAGACCGTAA ACTACGACGAGCGTGTCCGGCCCT GCTCACTGTGCTATGGTTACACACTGTATCT AGTGGGGATCGGGCGAAAAATGAA CCACATGTGTTIGCCATCGAAGAA GGTGAGATAATATTATGGGGGGGCTT GCCTTCATCAAAGGCGTGATAGGGA CATACGAGCCCAGAAAGCCCTGAA CCCGATCCGGGATATGITCAGAATG TAAGCAACTGGGGAACATGGGCAAC ATTGGATTTGACTCATCGACGCGG

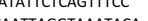
GTGGGTGATTGTCAGGGTTCCAAG CGTAATTGATGCCTCTTGCATTGG GGAAAATCGAGACCAAAATGTGGAAA AAACTTGTTGCCGGCTATCTTGGGG GGCCAAAAGACCCATCACTTAAAACAC ATGATGACTGGCCCGGAGTCAGATT GCAATCACGGATCTTGCTGTTAGG GCAACCGAGCGAATCTAATGACACC CCTACGGGTAAGCAGCTCAAAGGAGA AAAATCTCAAATCTCGTCGCAAAAGC
TCGGTGMTGATTIGGTAAGGGT CCAGTTGGTCTTGCCAACGAAATG AAGGCCACTGAGCGGTGAAGAGGGA
TGGTCCCTTAAAGTAATAATCAGCG GCATGGGTATGCTGGATTCGAGTG CAGCAACTGAACTAAGGACCCCCA GCTGGAAAATGGAGCTTCCCGTTG ATGCCACGCCCACTCCACACC
ACCCAATGGAAGGTCTGCTTGG GCCACCCACATTCTCTCTCTAACTCGG TGTAAAGTTGAAGAAGACGGTGGATTI ACACTCACTGCTGTTCCCGTCC CAACAACGATGGTTACTAATAACTTTAAAT CGAGGGGCTTAGTTIAGGAAGGGA TGGGACACTTGAAAGCGAAGAGCA cCCCTCGAAGGGAAGGAAACACAA cGGGAGCAGGATGAAAAAGGACGAG AGAGATGGCTGGCTGATCGCTTTIG TCTGGGGATCAAGGTTTCGGTTTG CGATGTTGCGTTGAAGCCTGTTA TAGCAACCCATCTGGGGTTTGGGT ACGGAACCGGAGAATAAAAACAGAAAA
GCAGTGCAGCATGGAGATAAGT AaCGGCACAGAGAACGCAACGAA ccGcCAgctctgCtgGagagaAa CCCCTTTIGCCCACTCCTCCATTI TCCTACAAACAGCCCATCAATCAACG
CGTGGCAAGTCATTAGTGGAAG
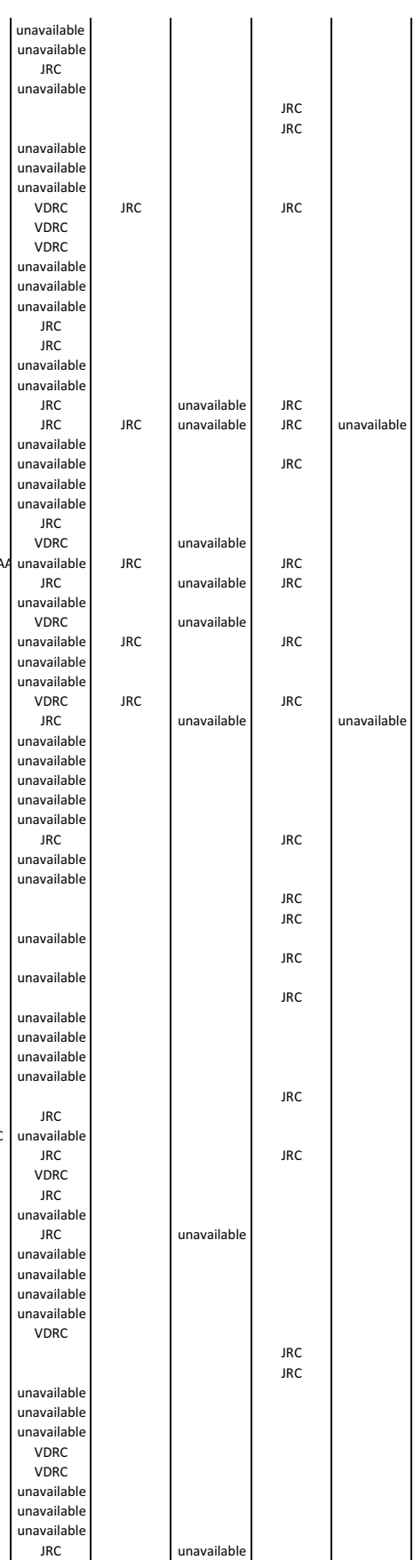

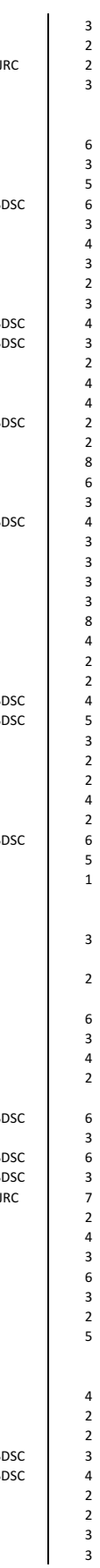

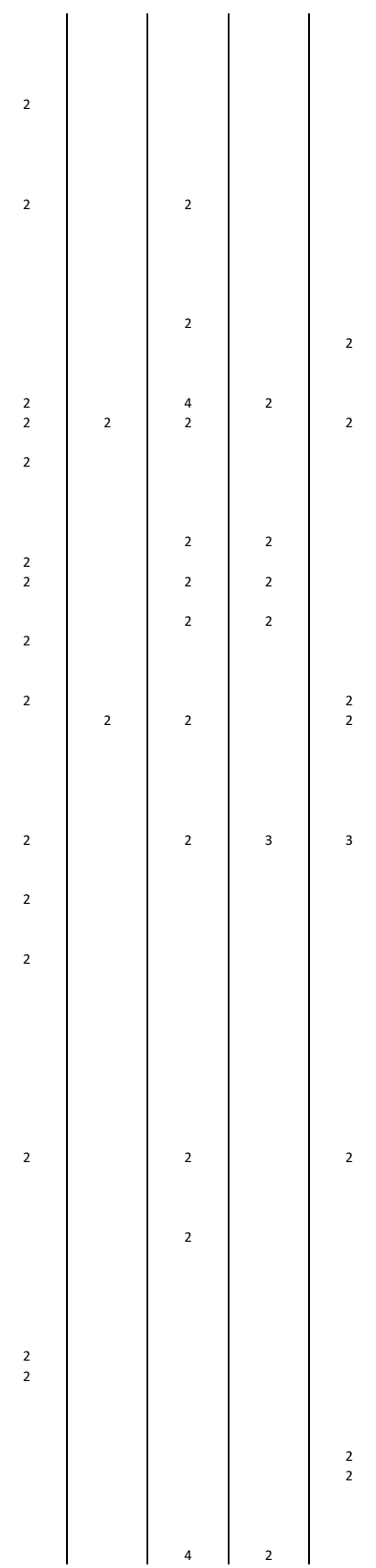




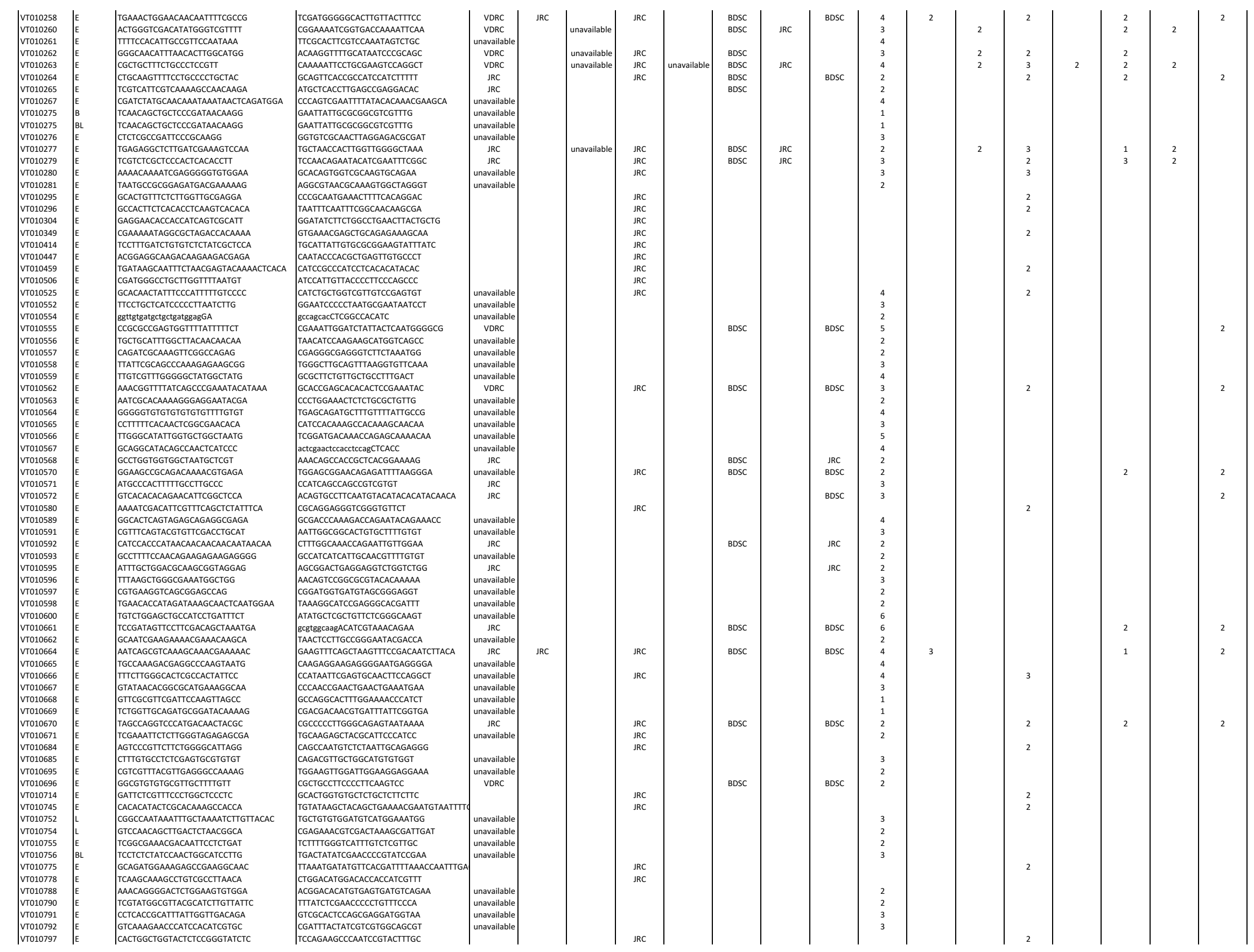




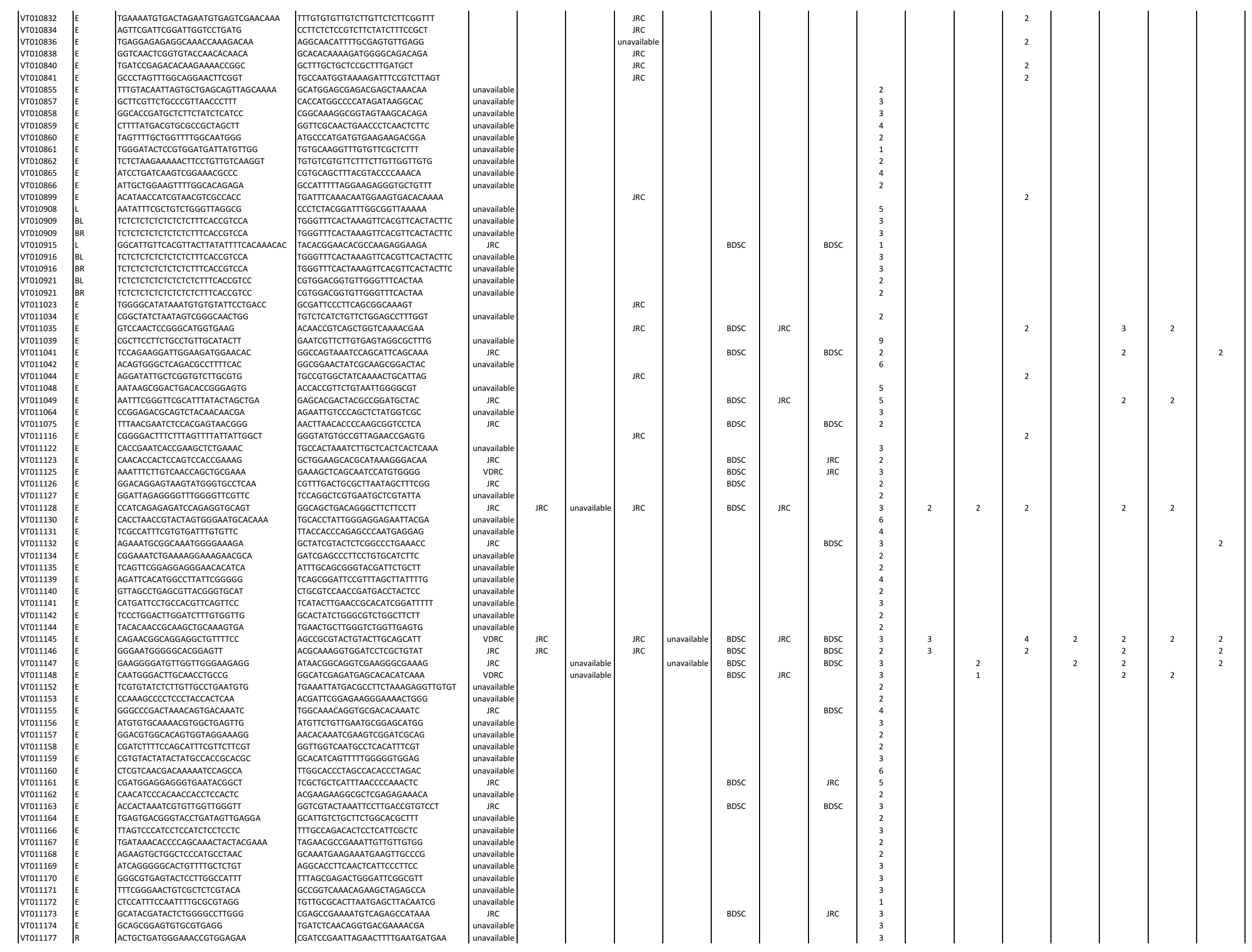




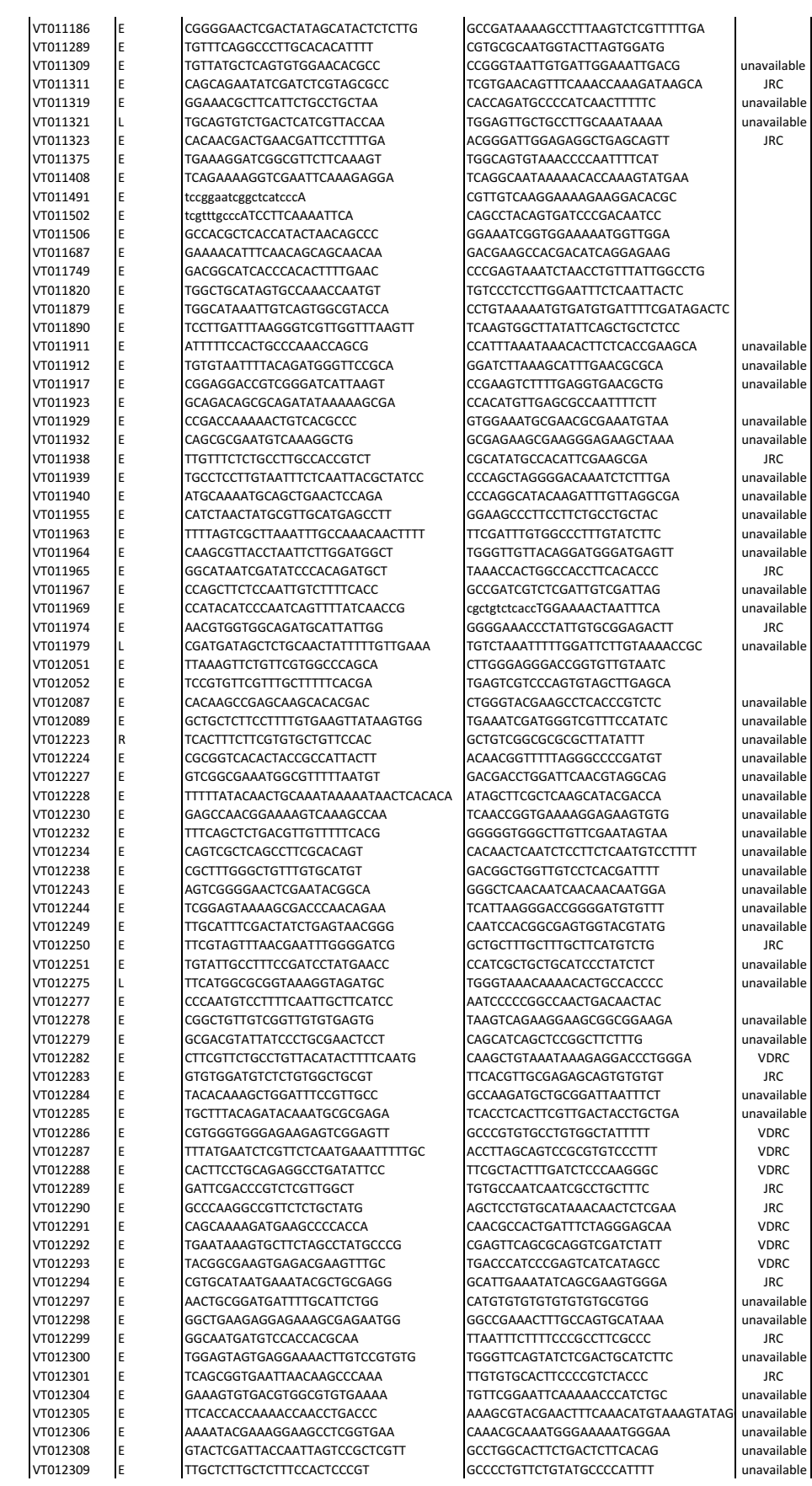
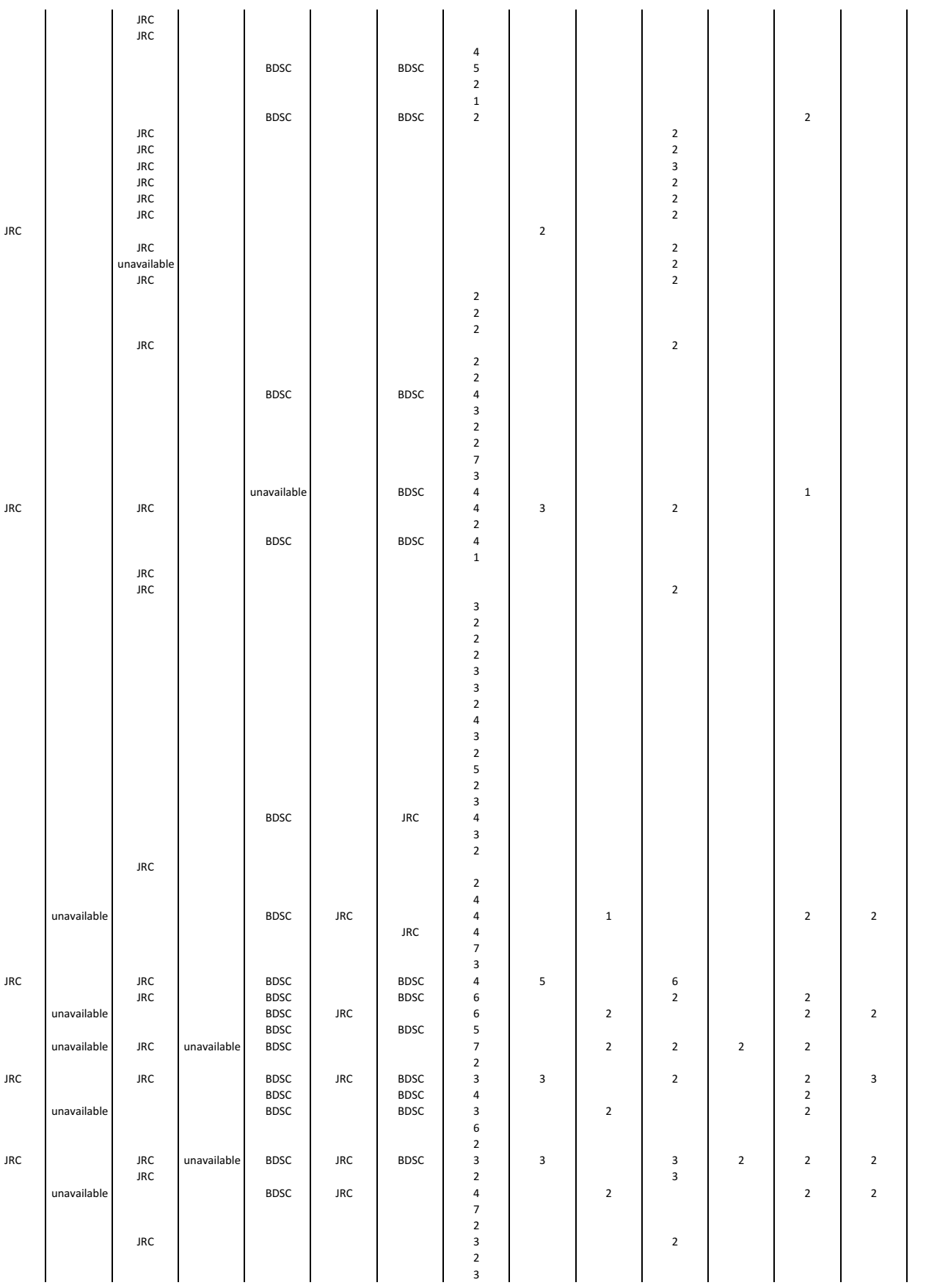


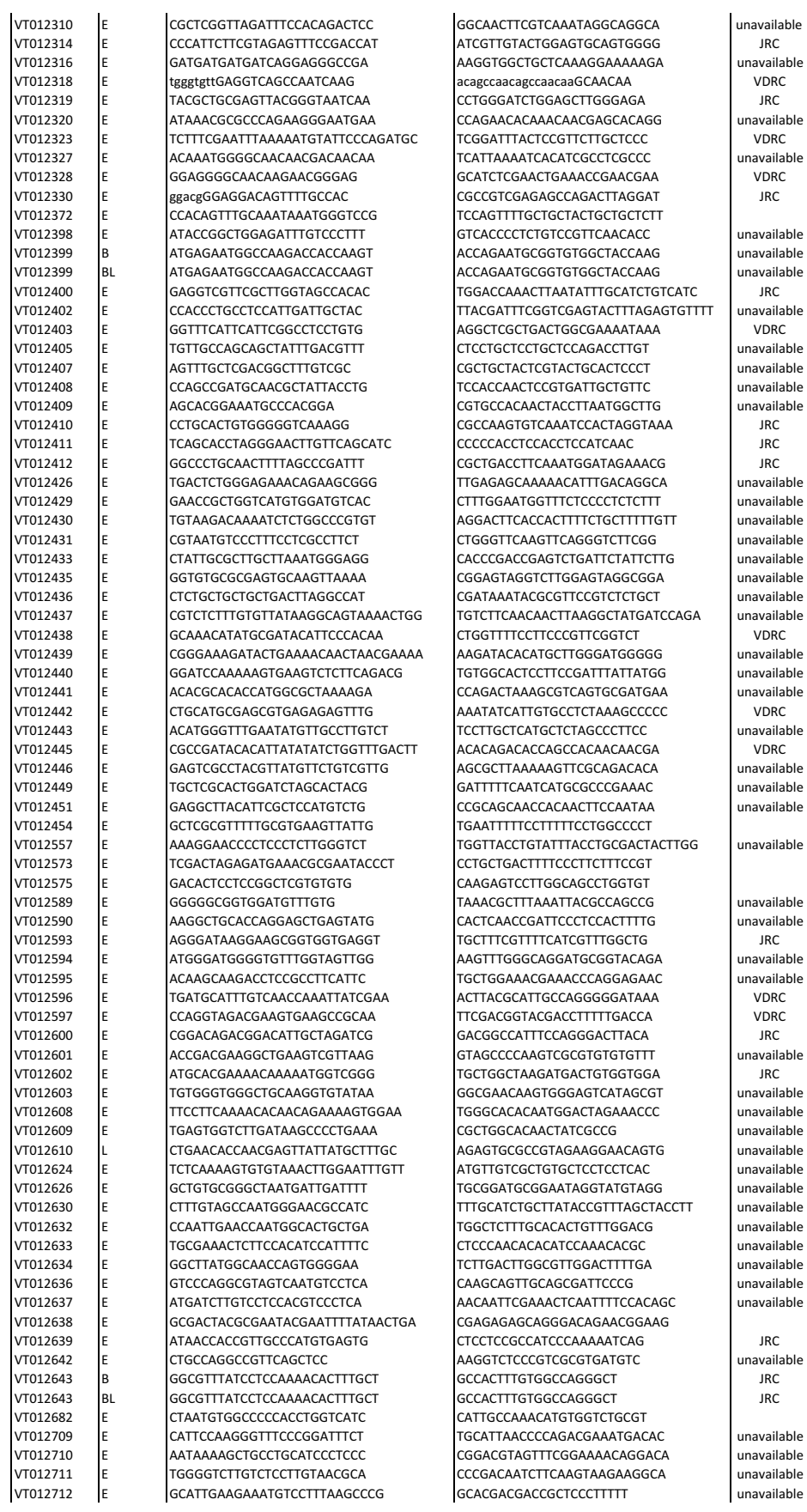

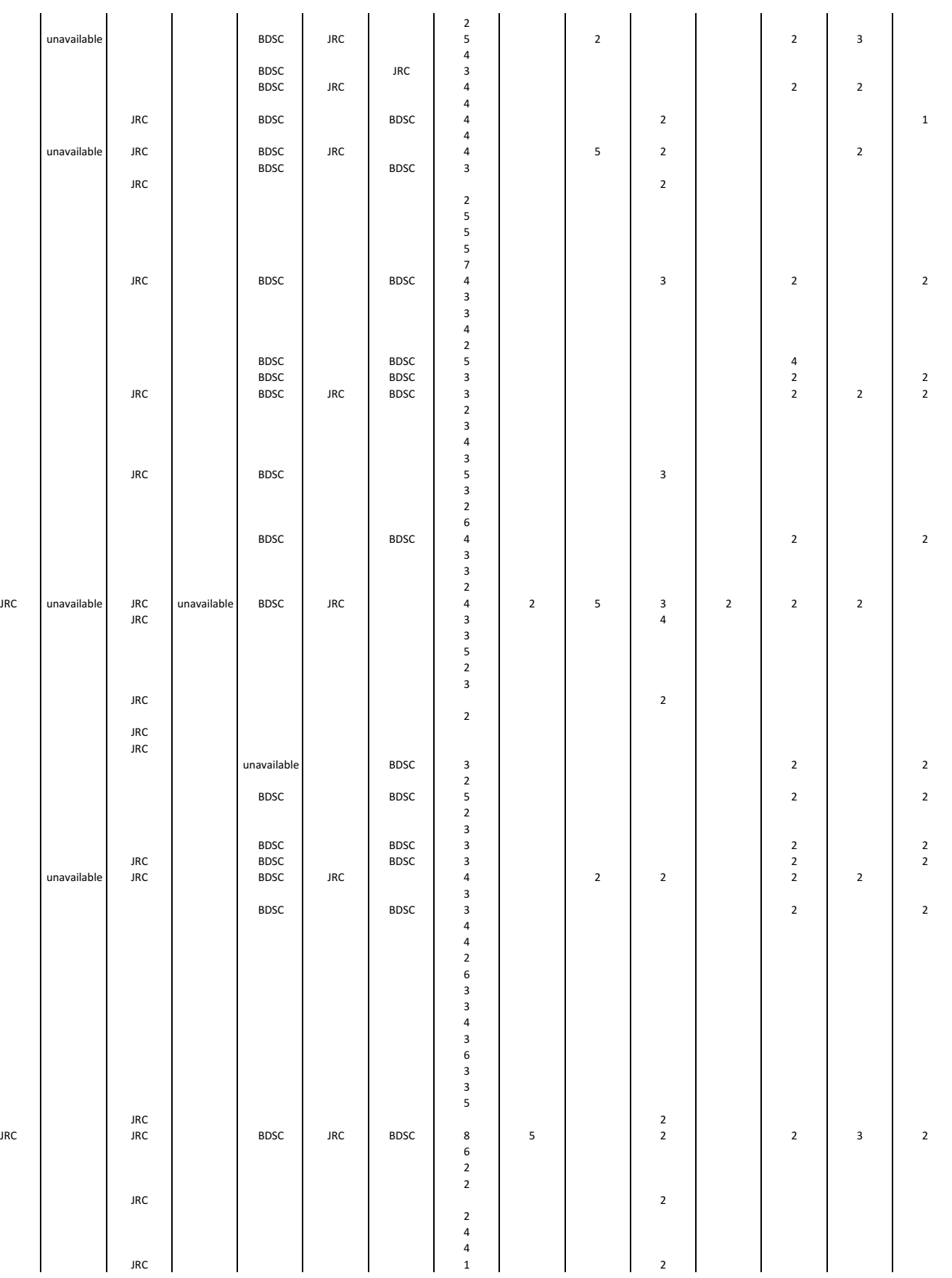




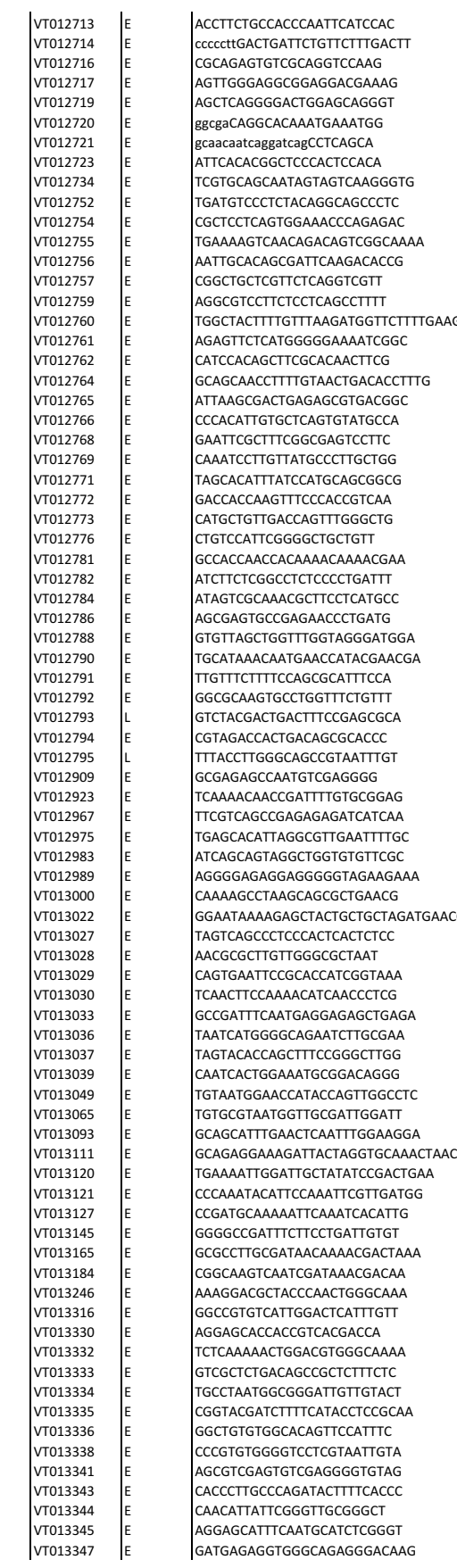

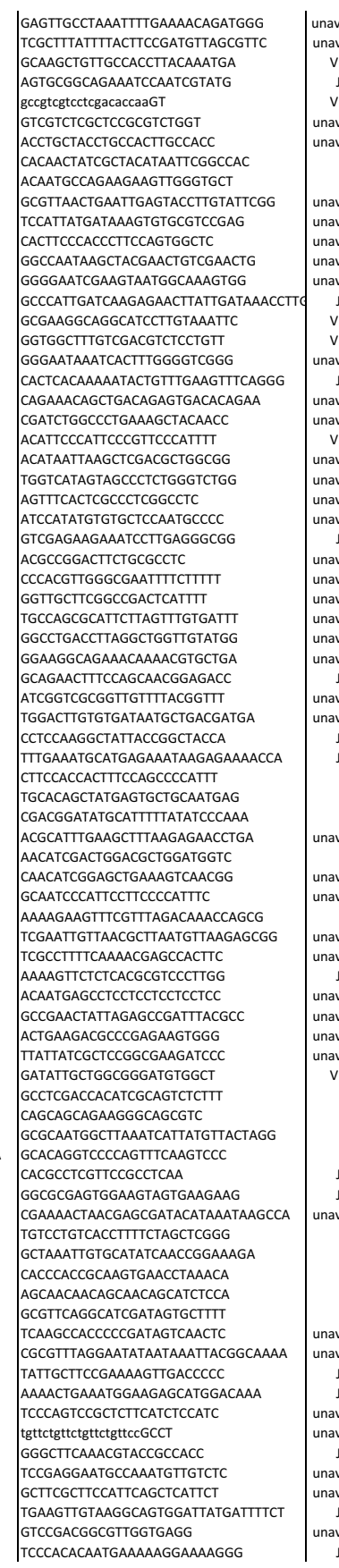
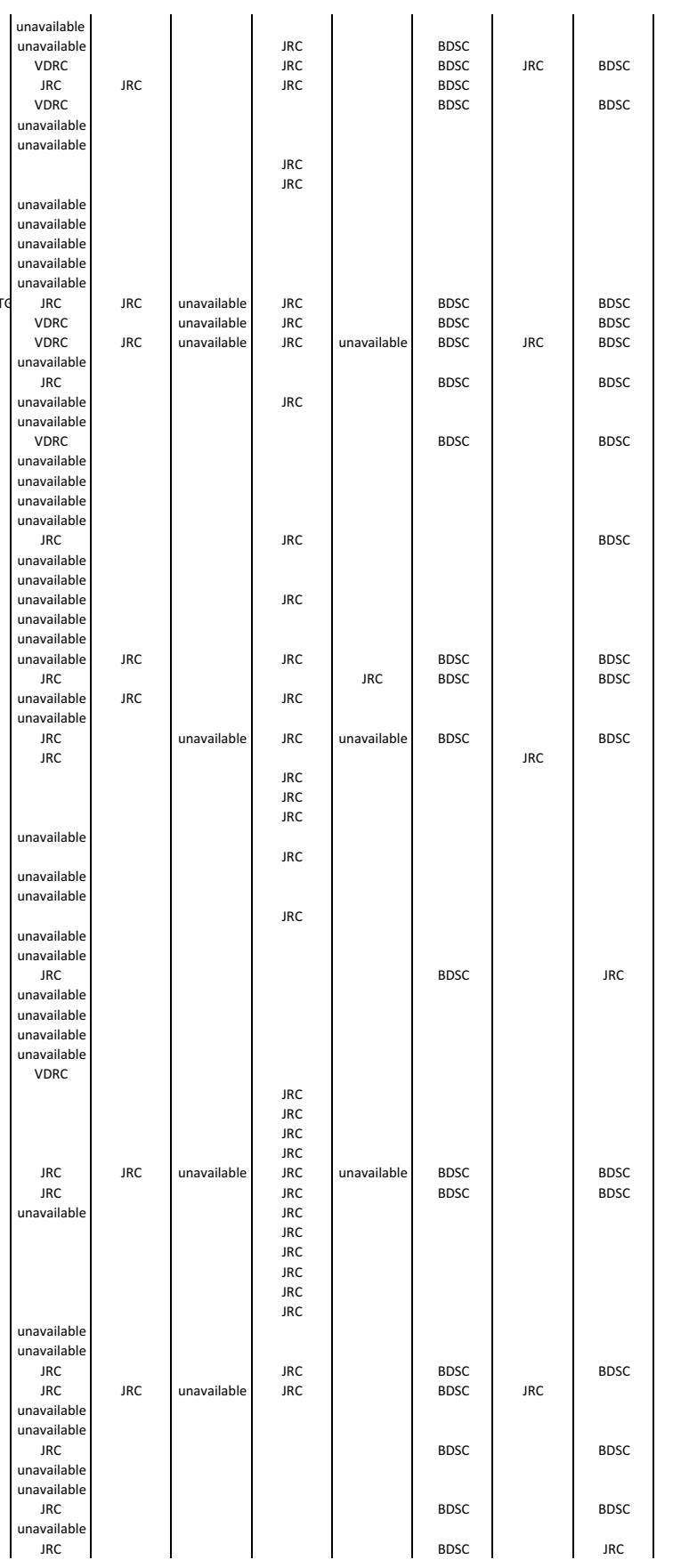


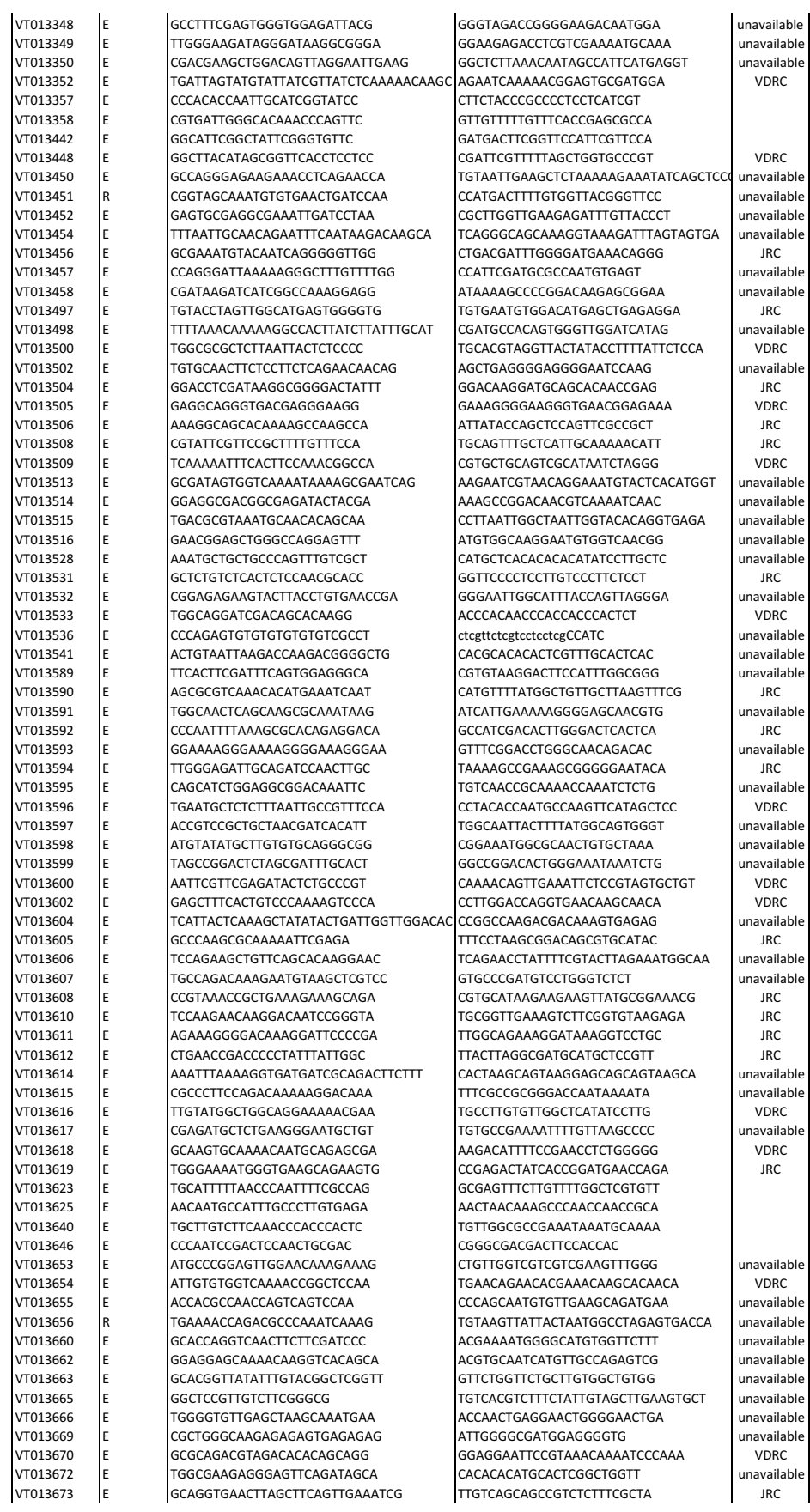
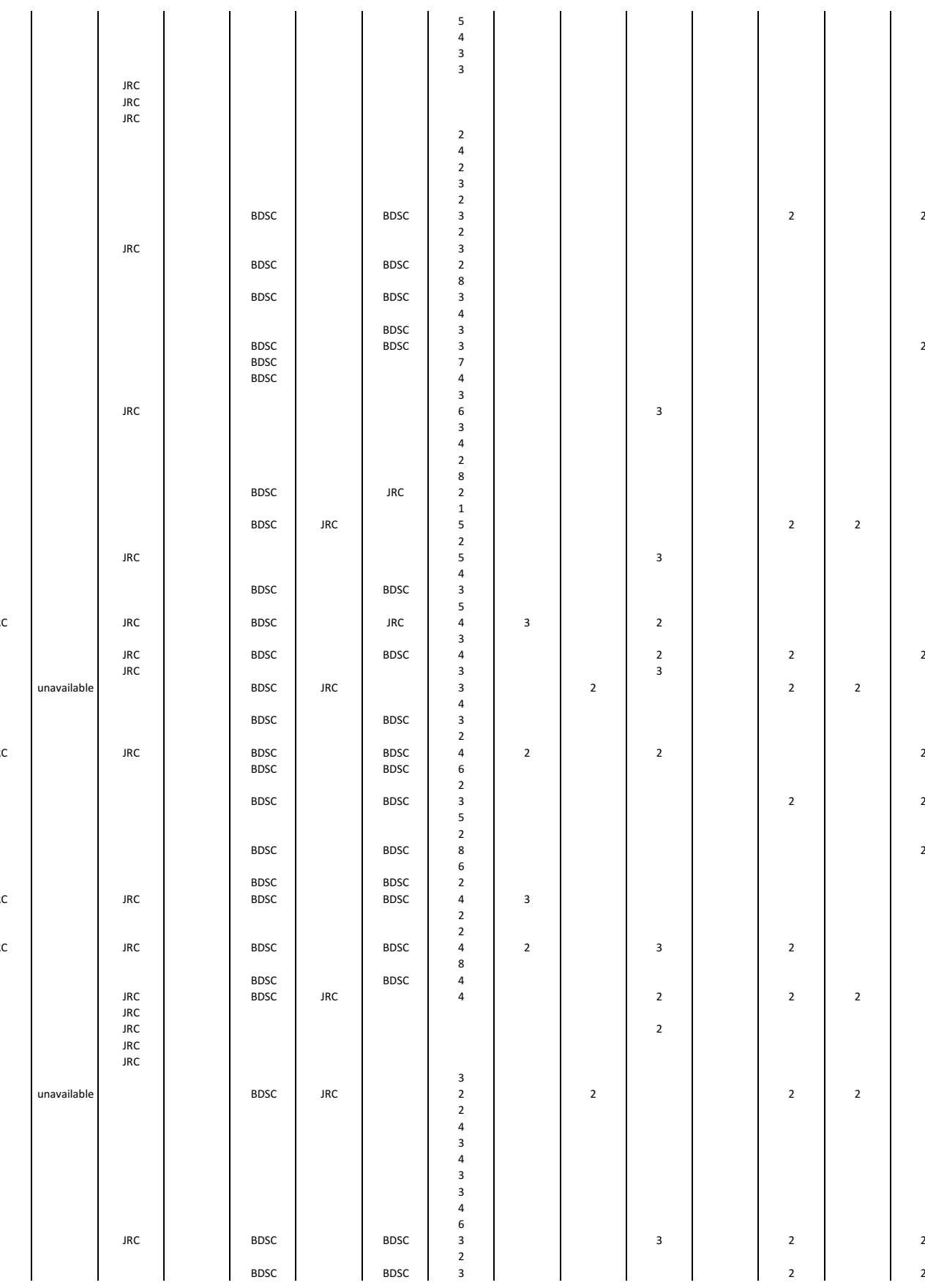


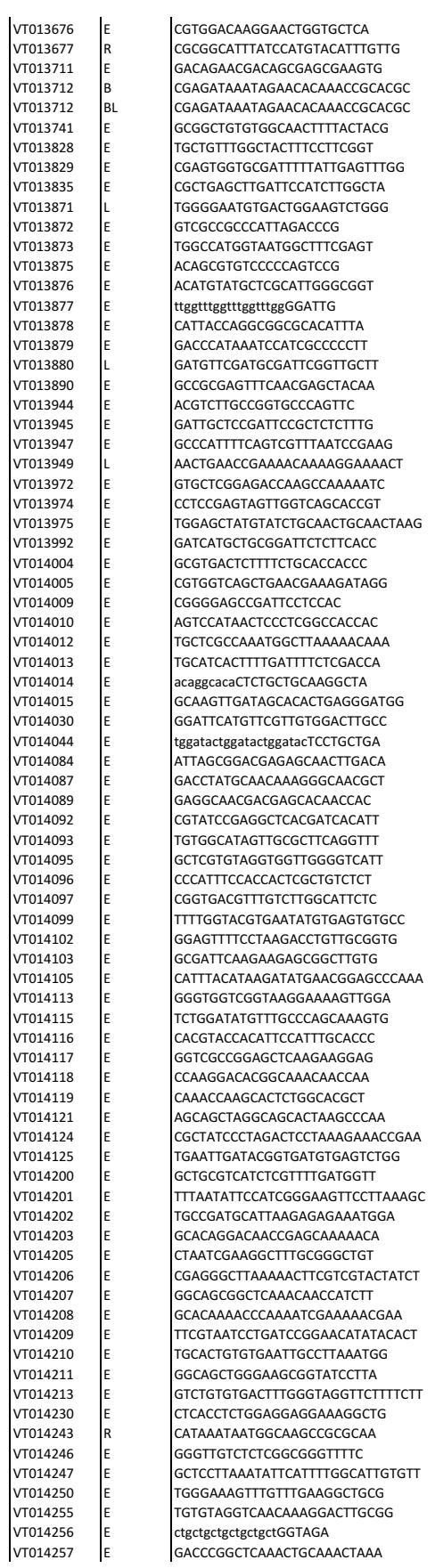

\begin{tabular}{l|l|l} 
VT014257 & E & ctgctgctgctgctgctGGTAGA \\
GACCCGGCTCAACTGCAAACTAAA
\end{tabular}

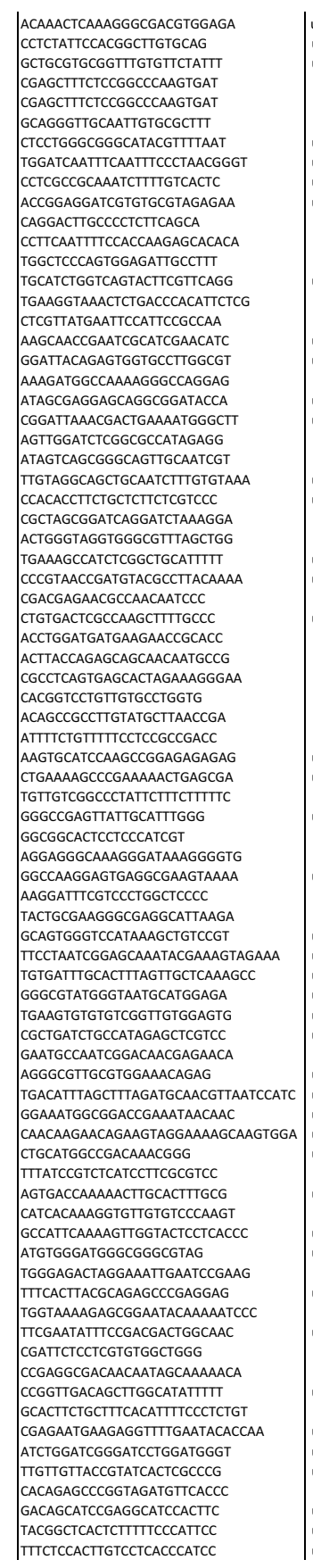

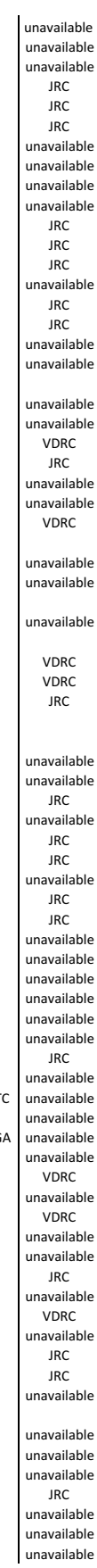

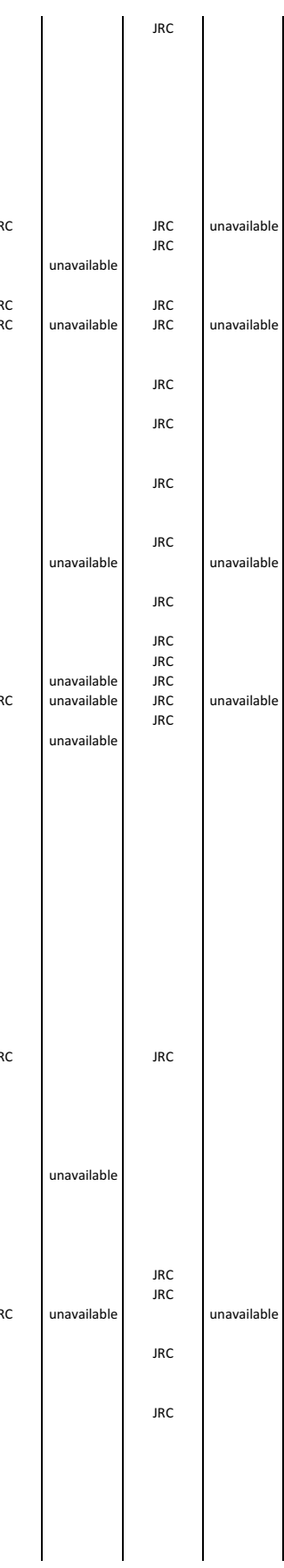

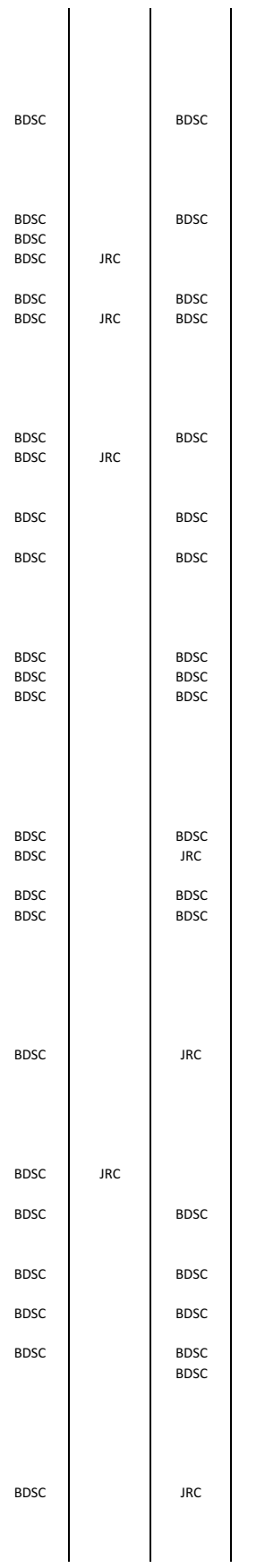

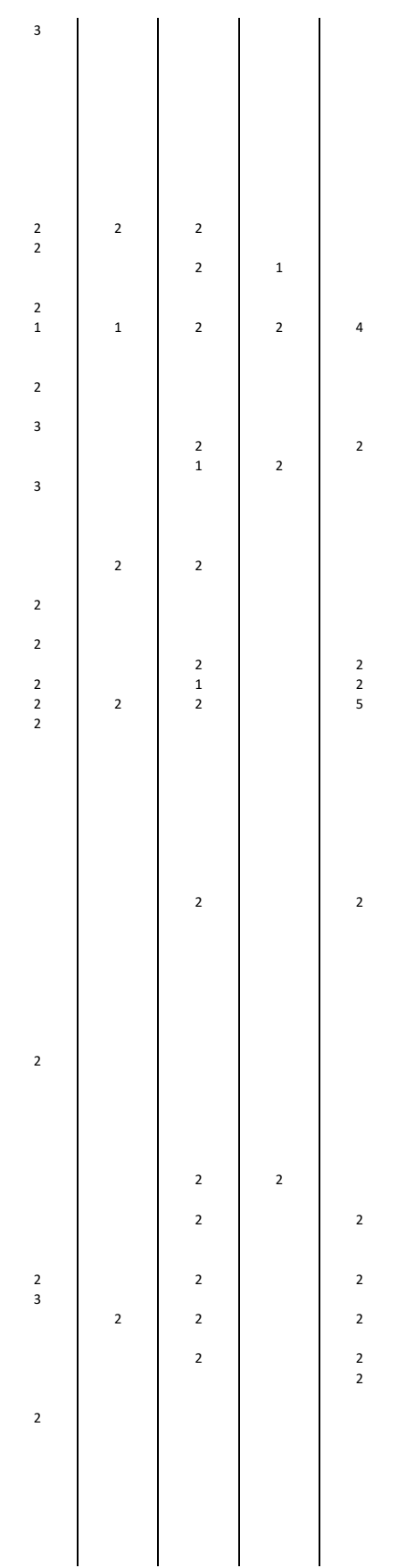




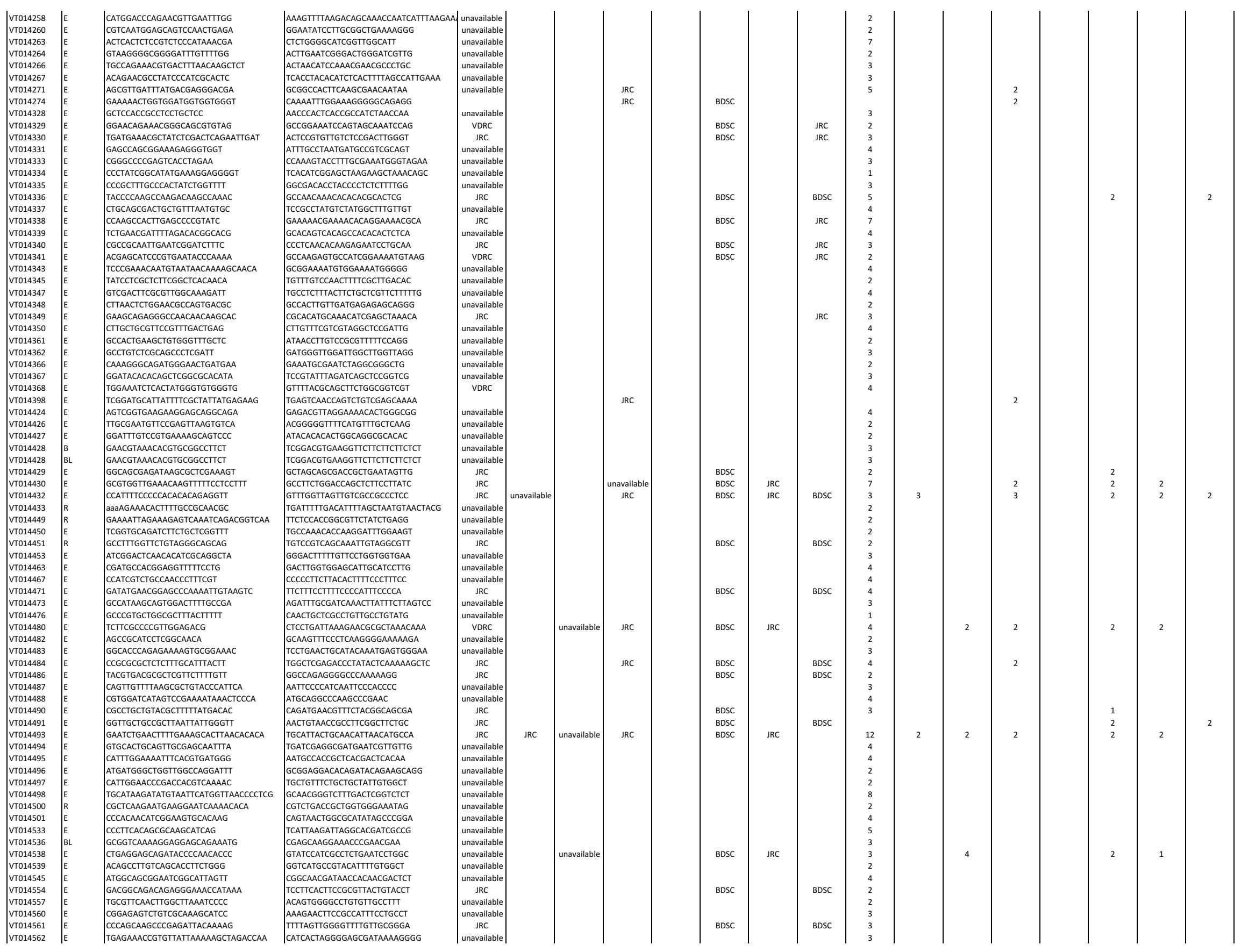




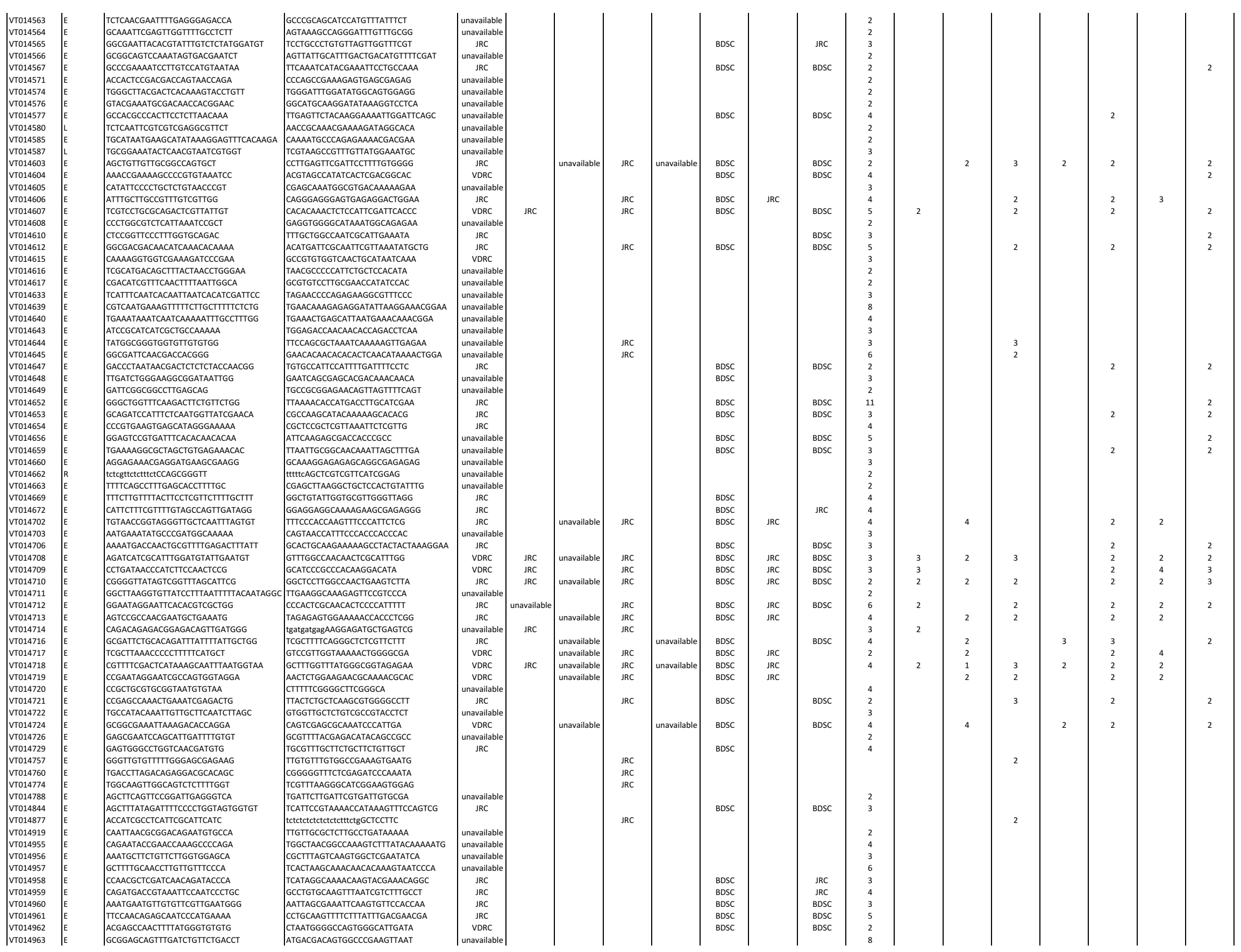




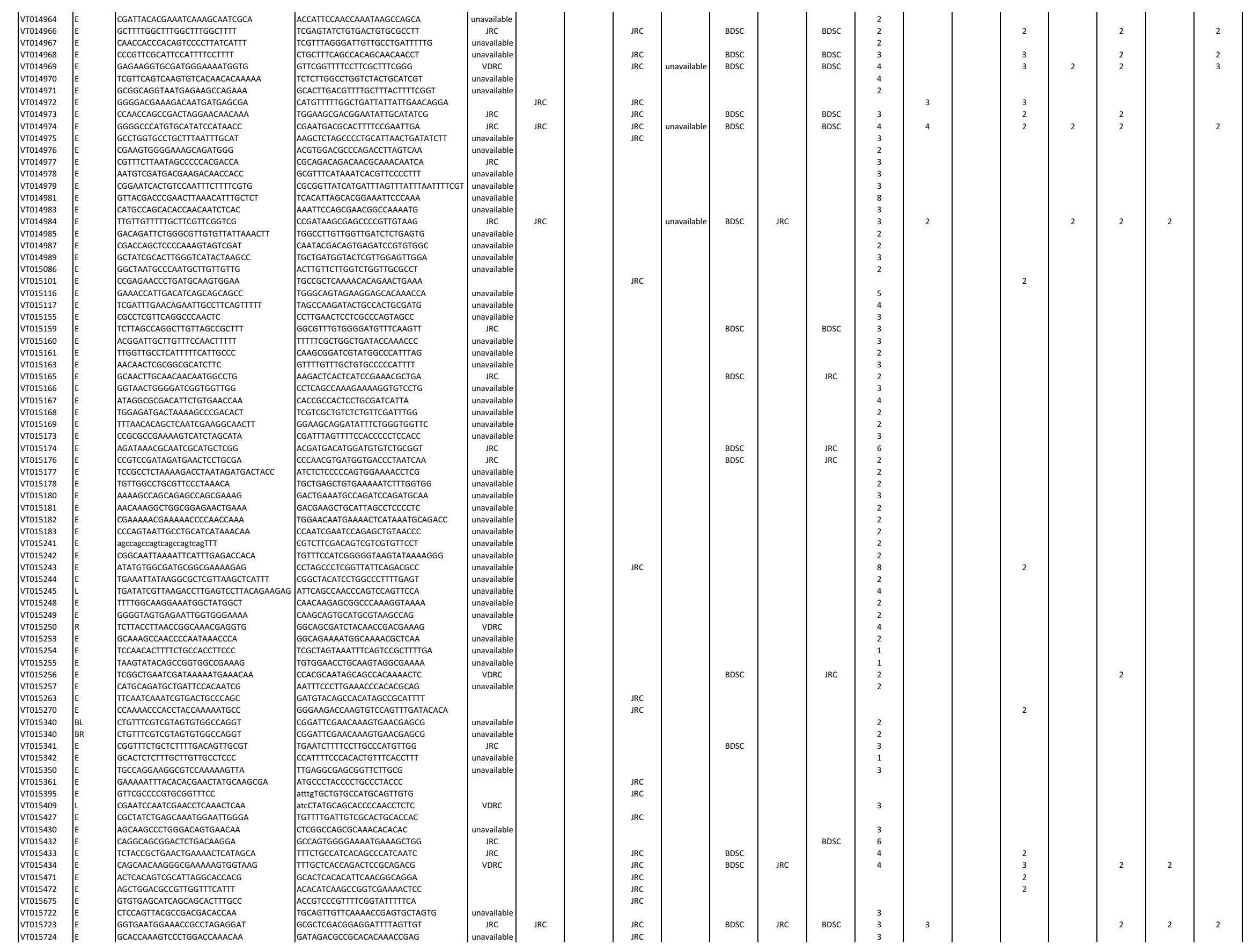




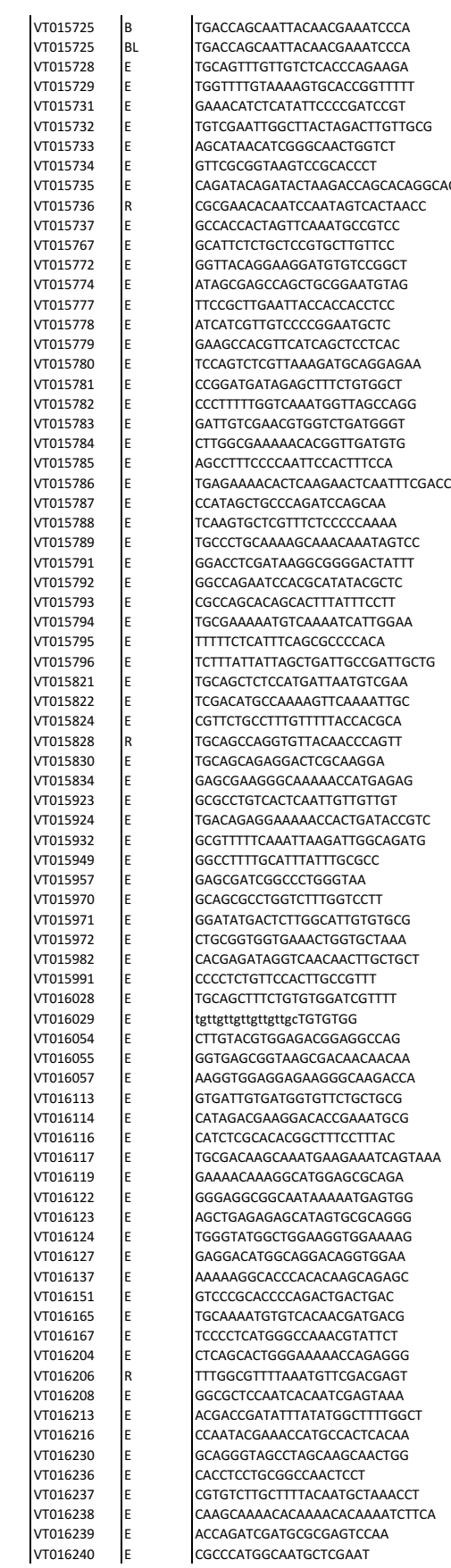
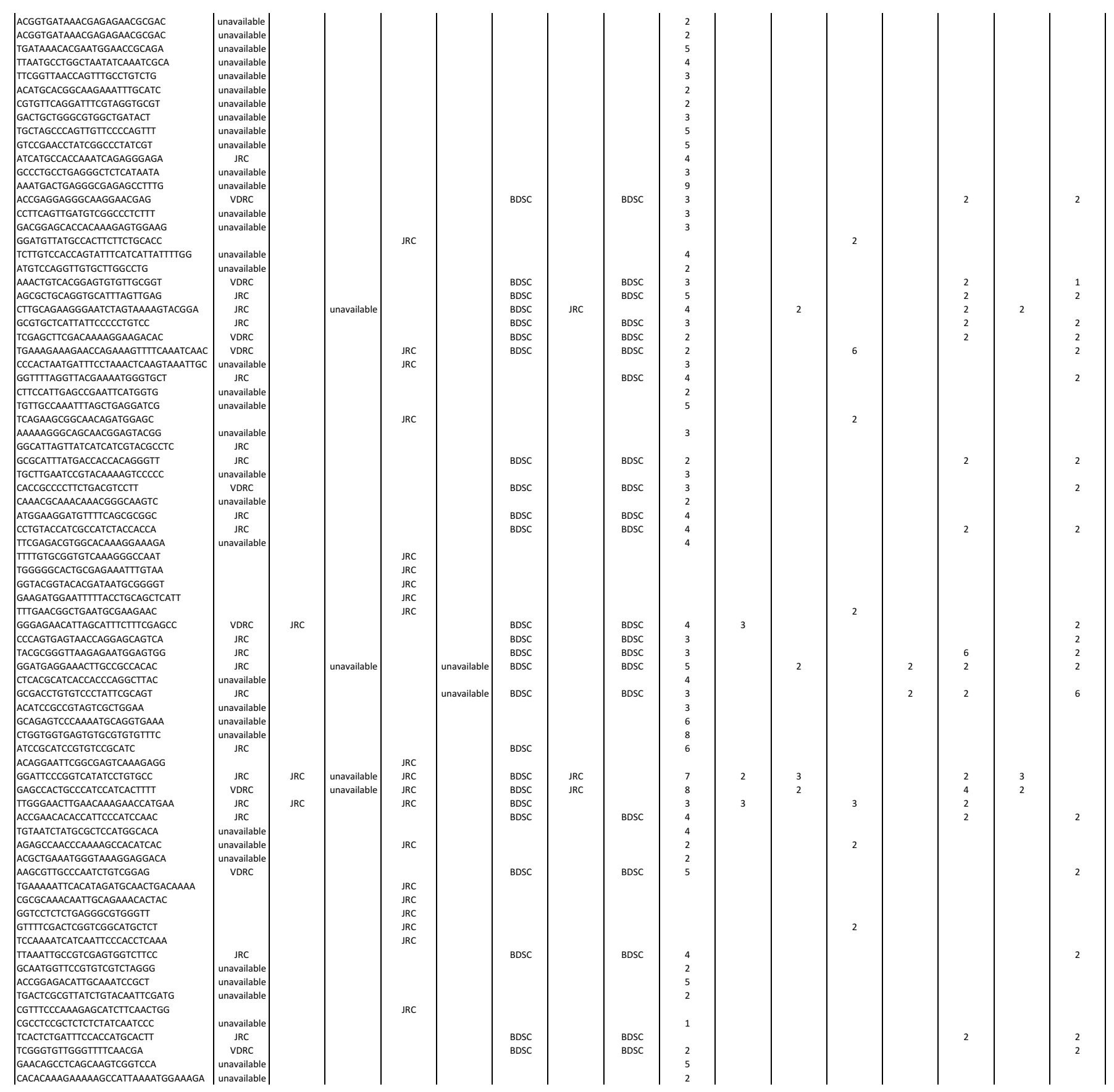


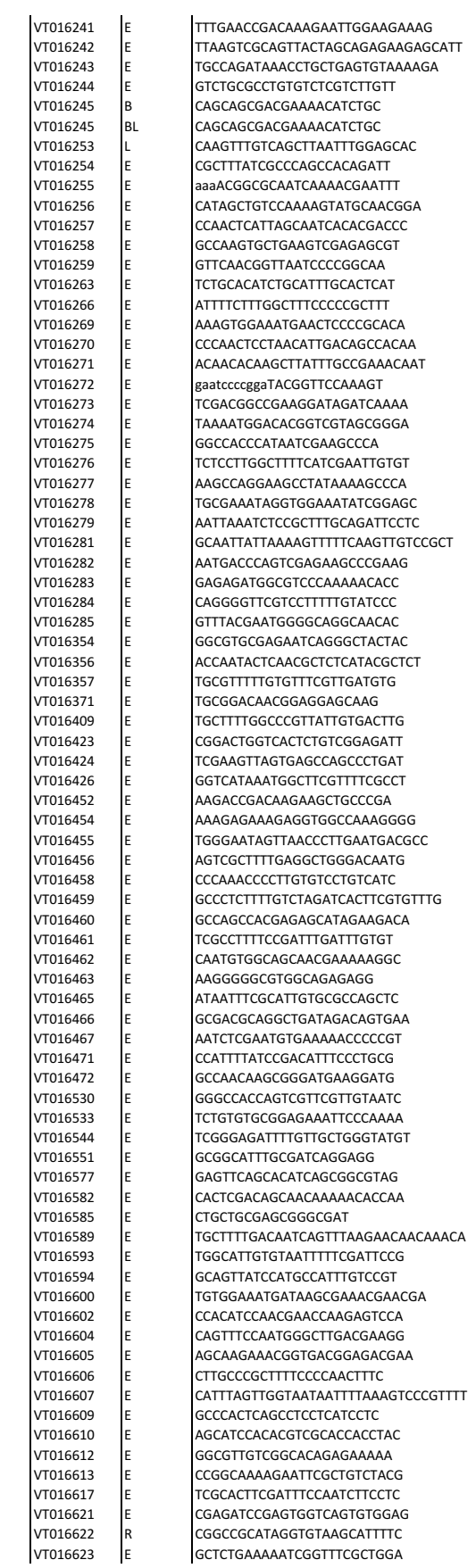

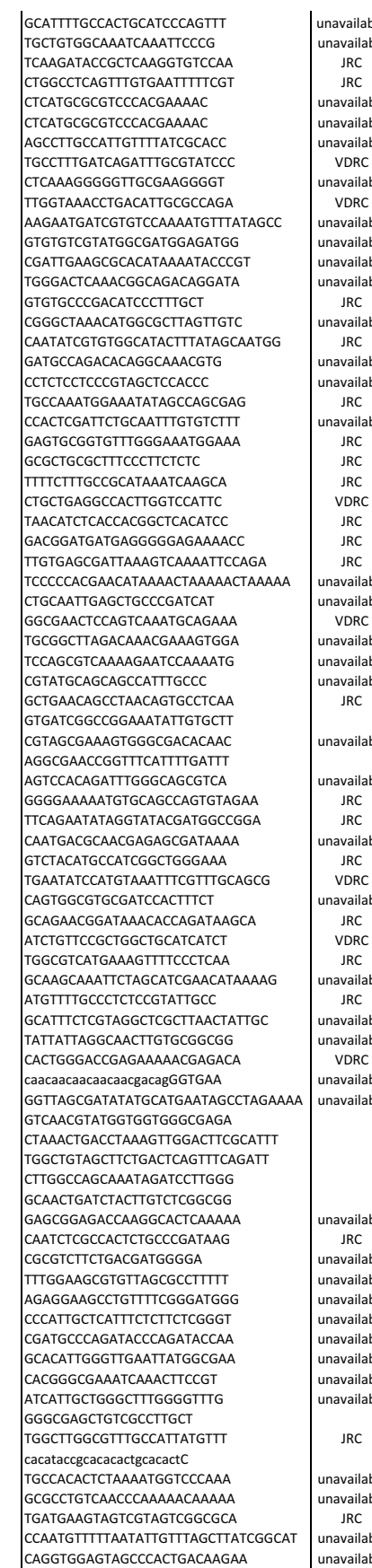

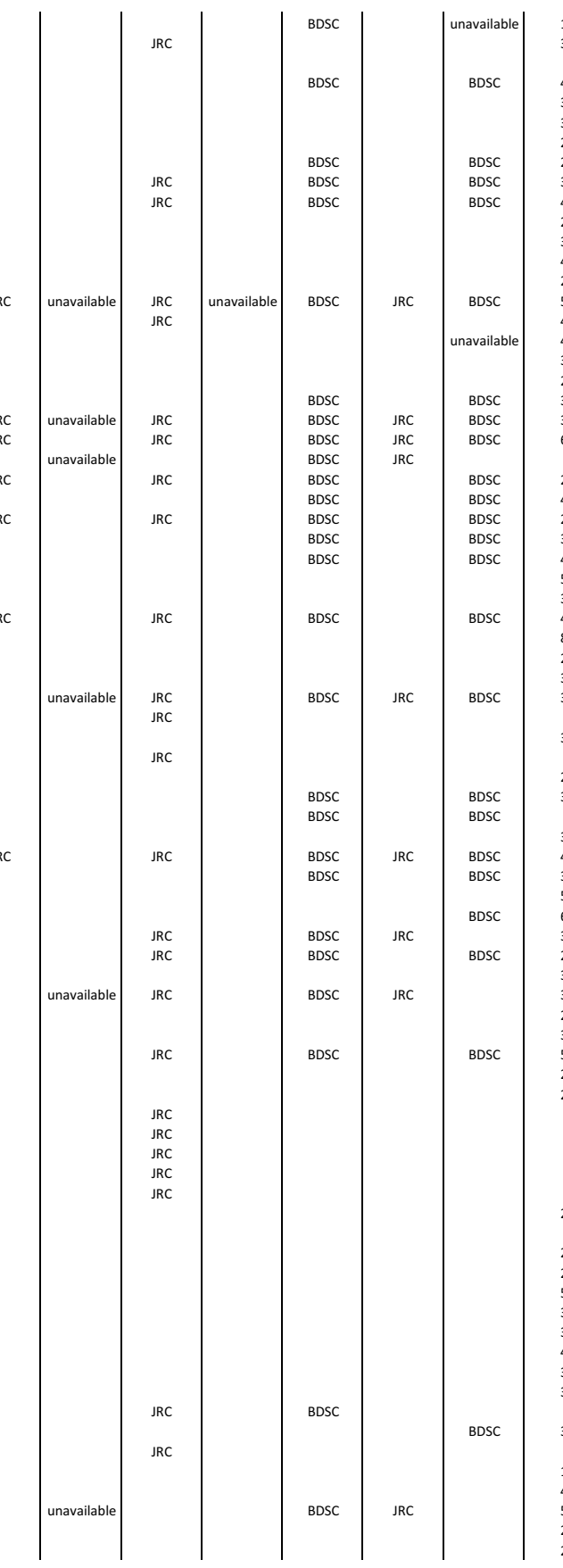




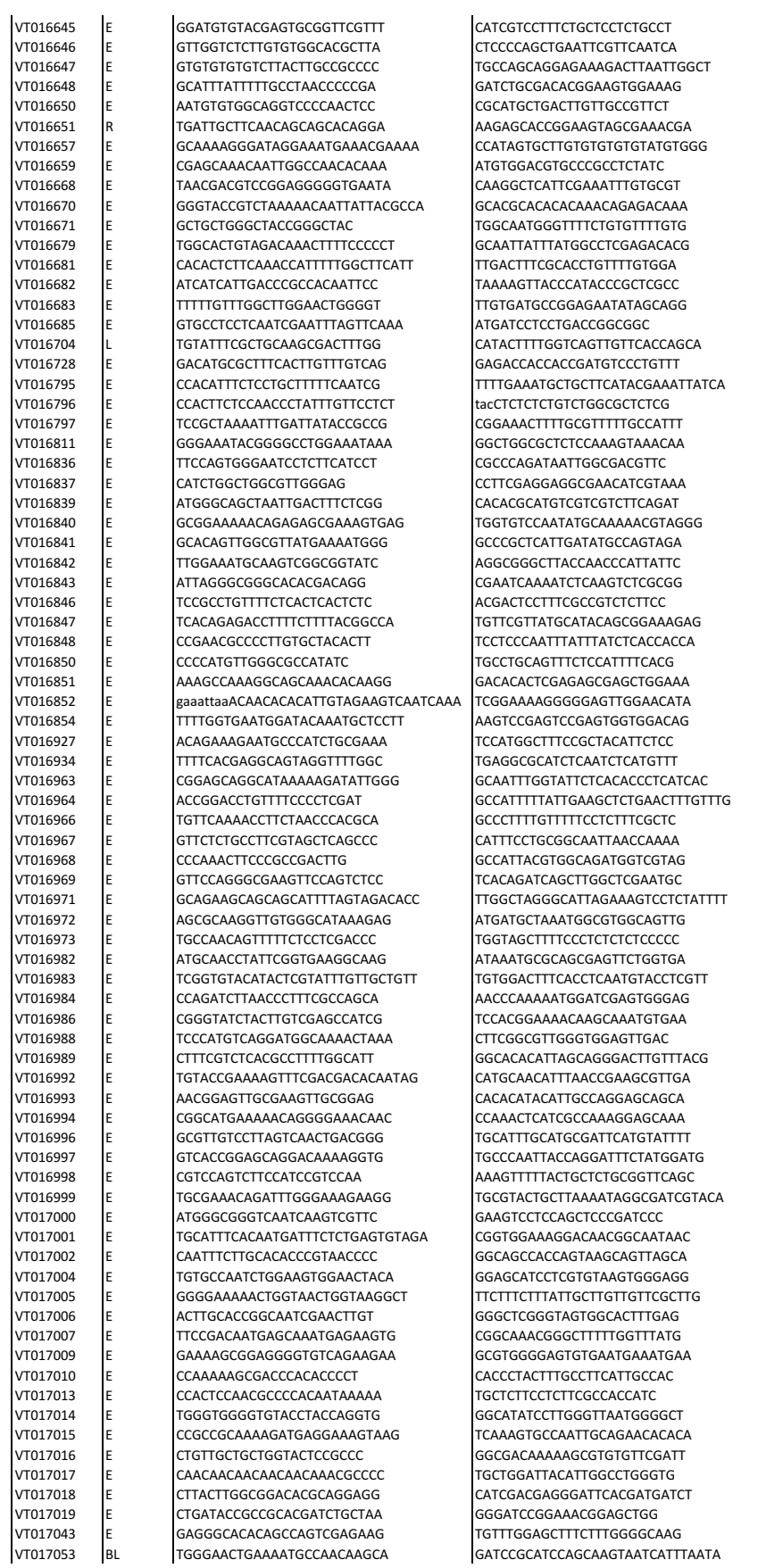
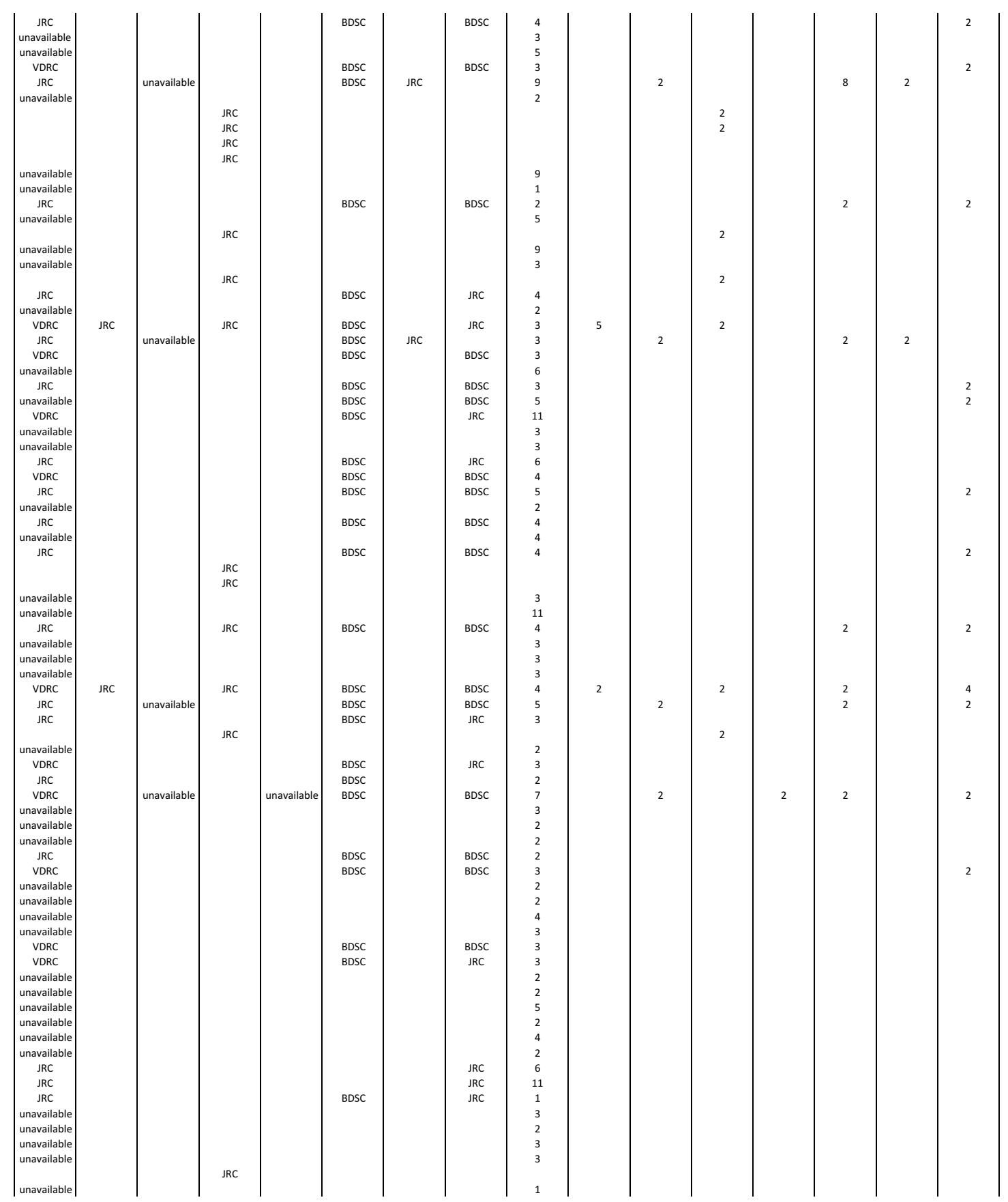


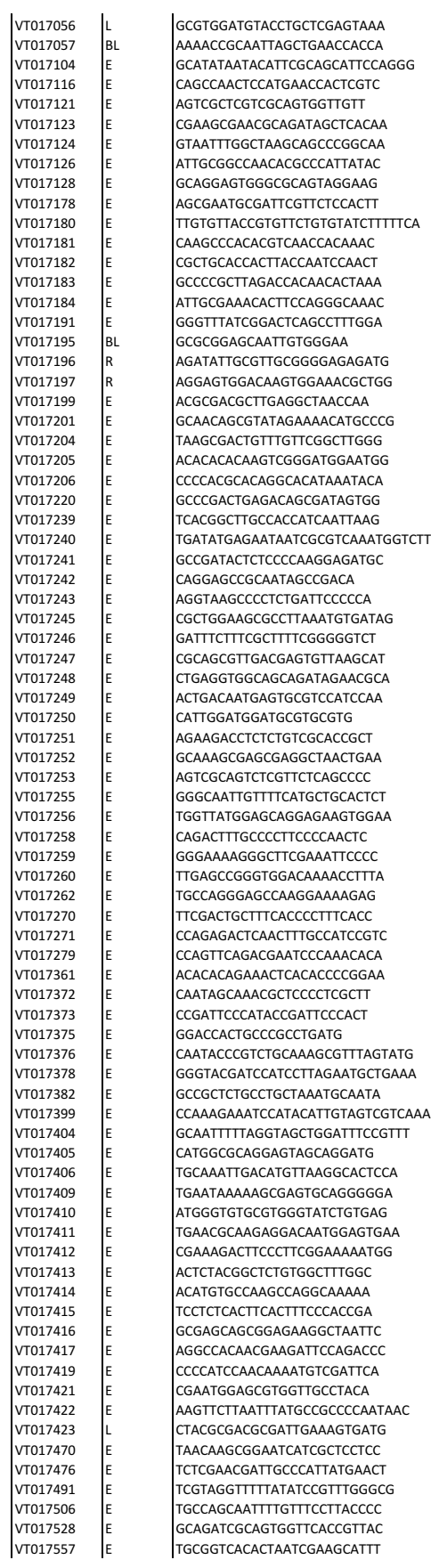

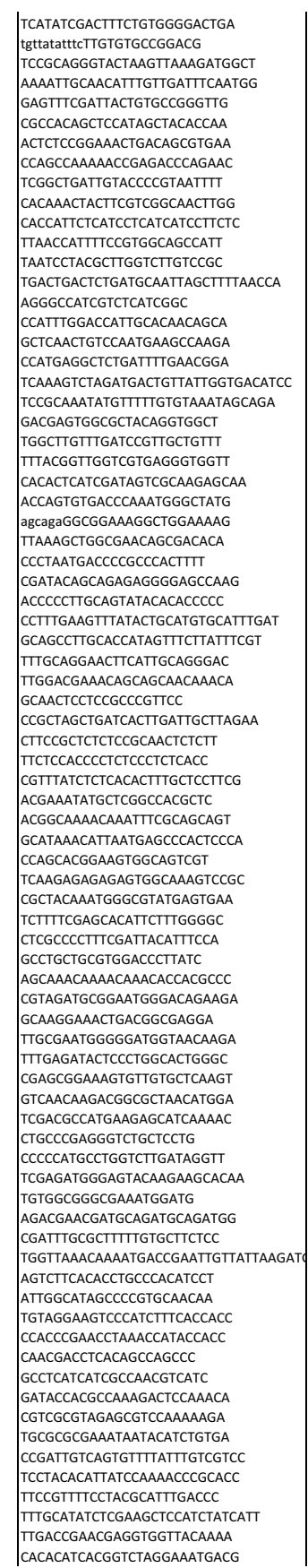
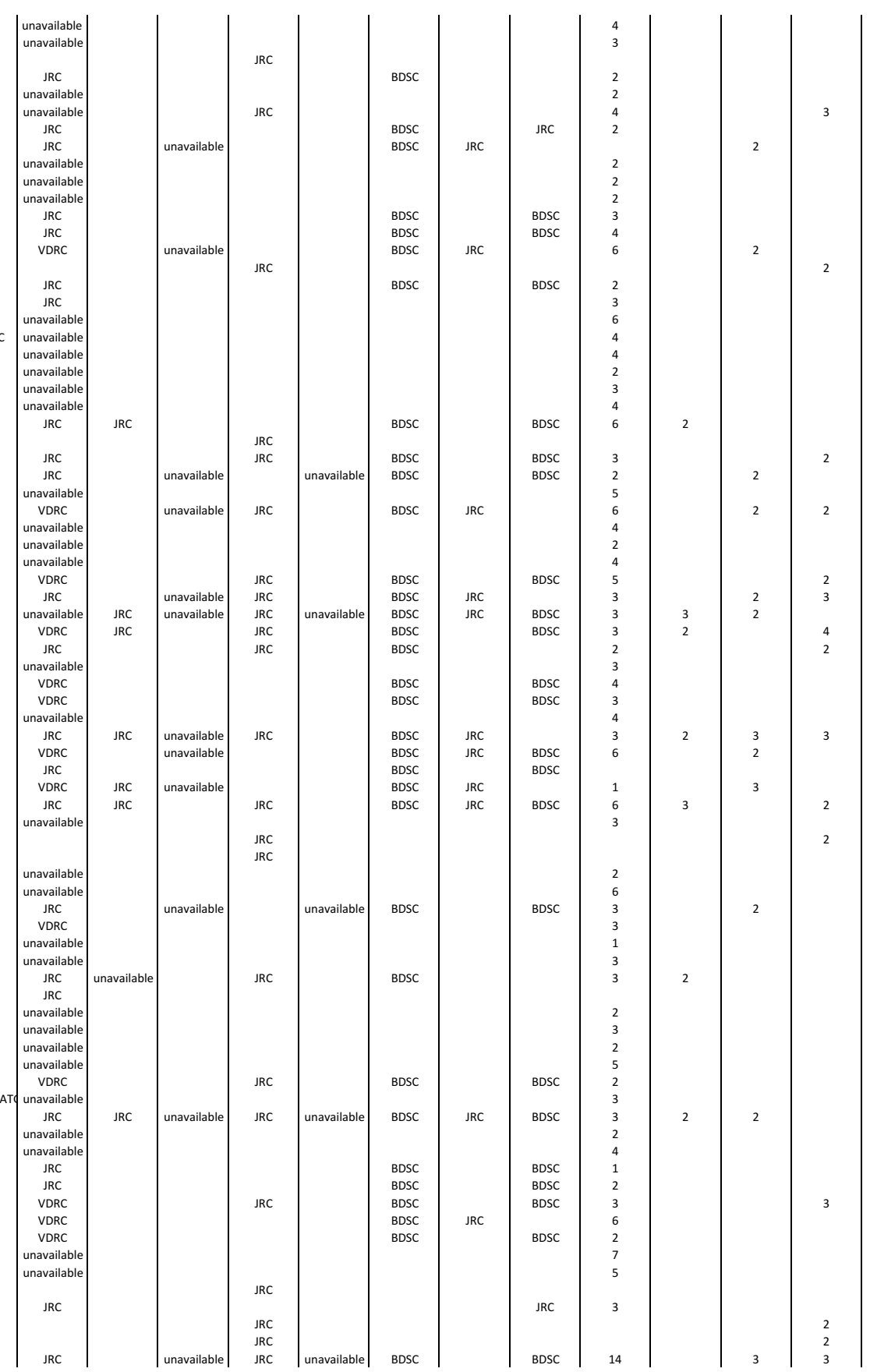


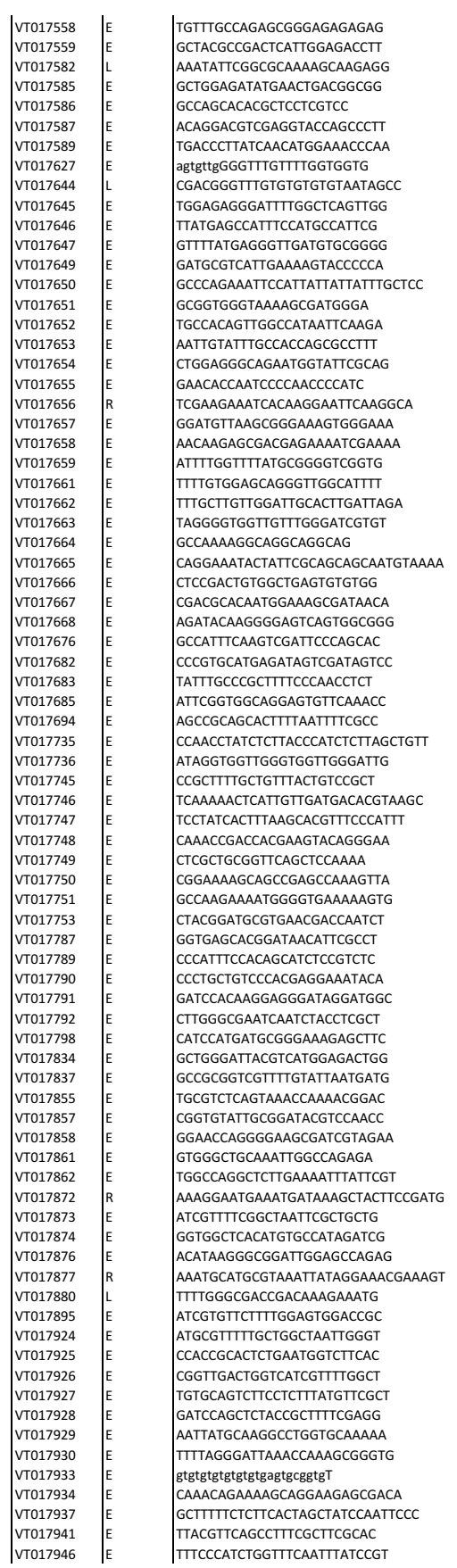

\begin{tabular}{l|l|l} 
VU017941 & E & TTACGTCAGCCTTCGCTTCGCAC \\
VTT017946 & E & ITTCCCACTGGTTTCAATTATCCG
\end{tabular}

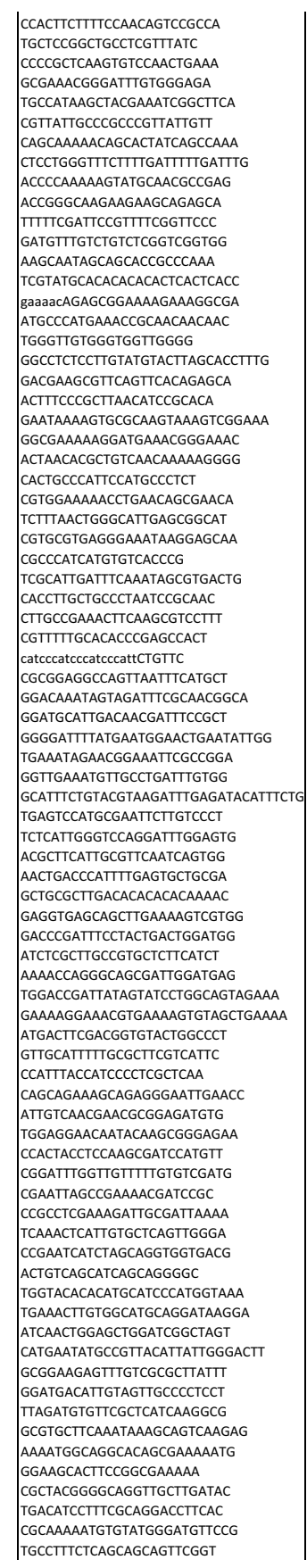

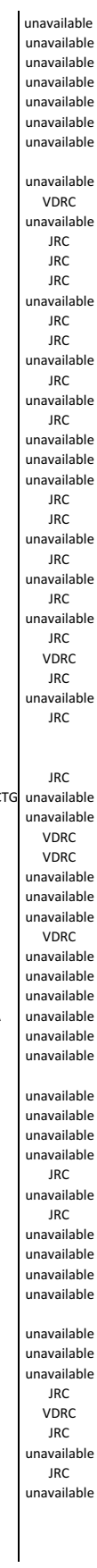

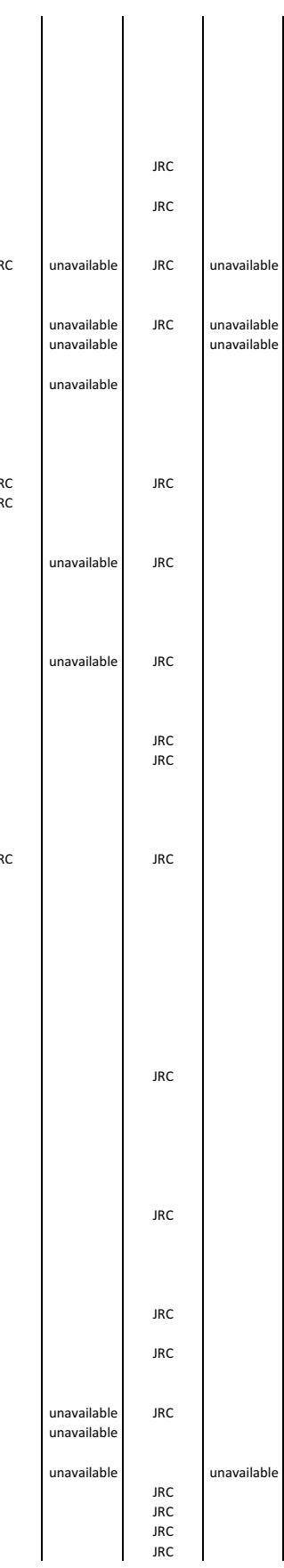

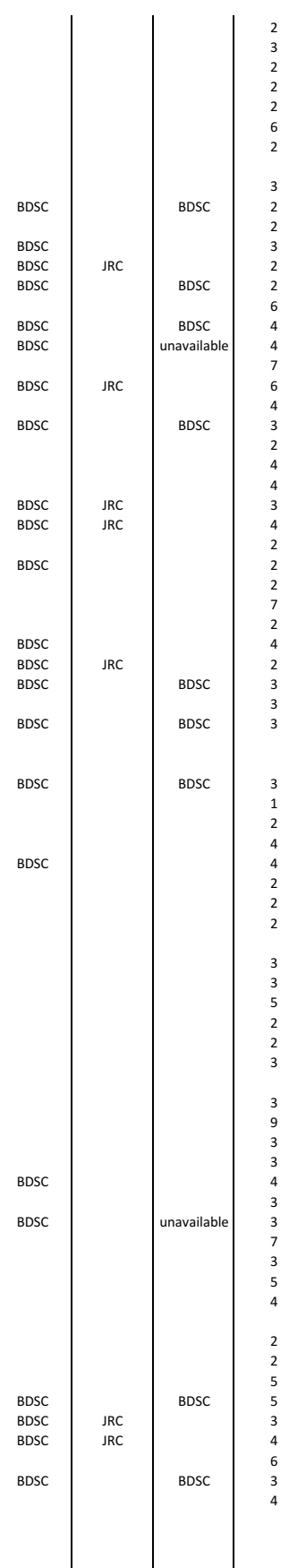

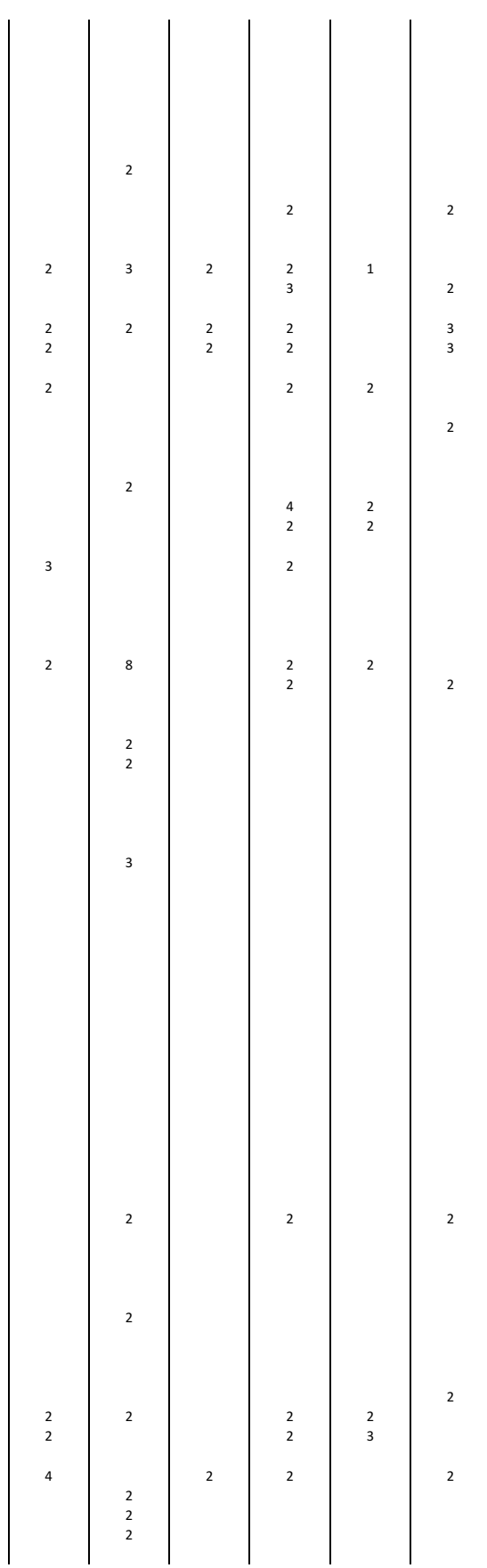




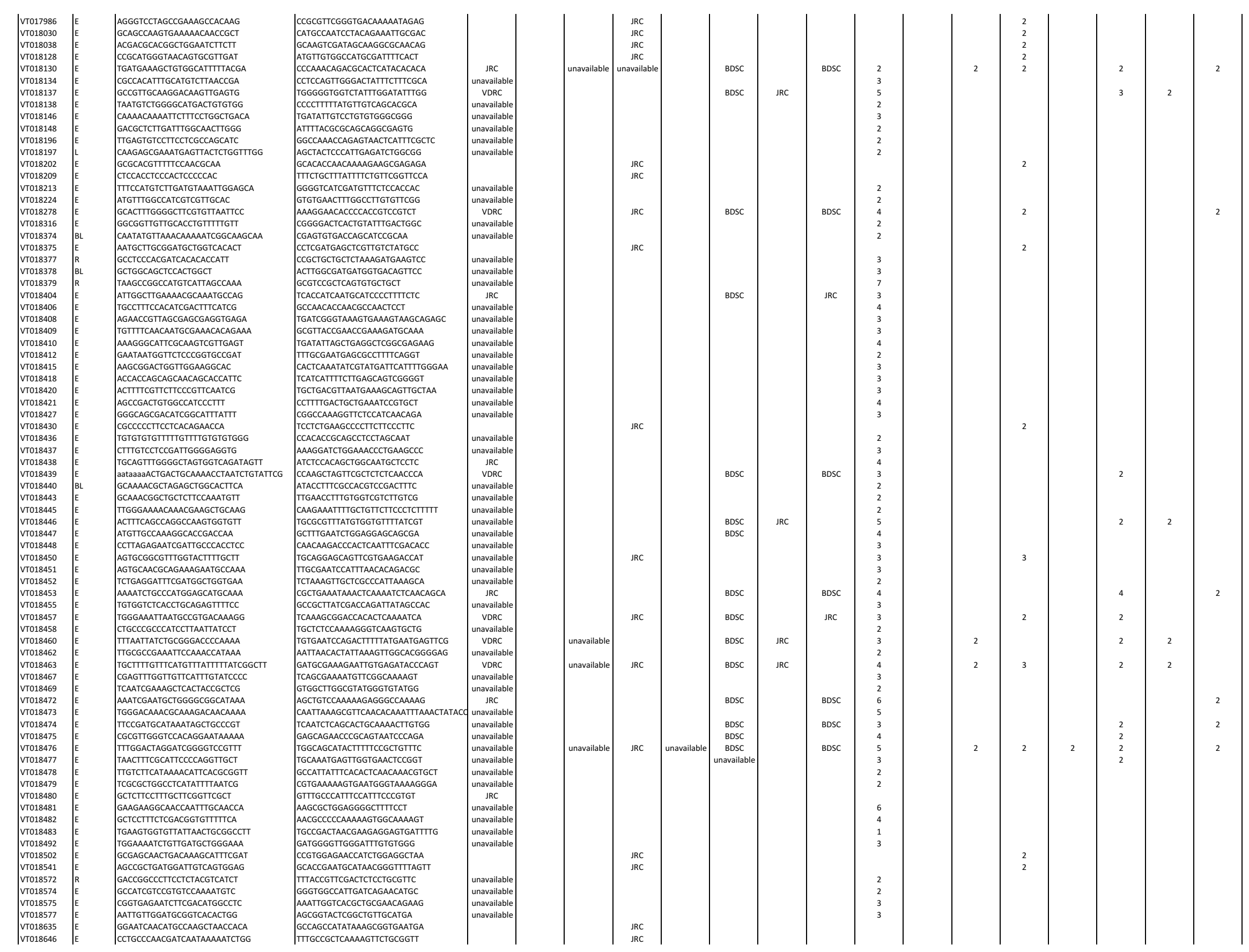




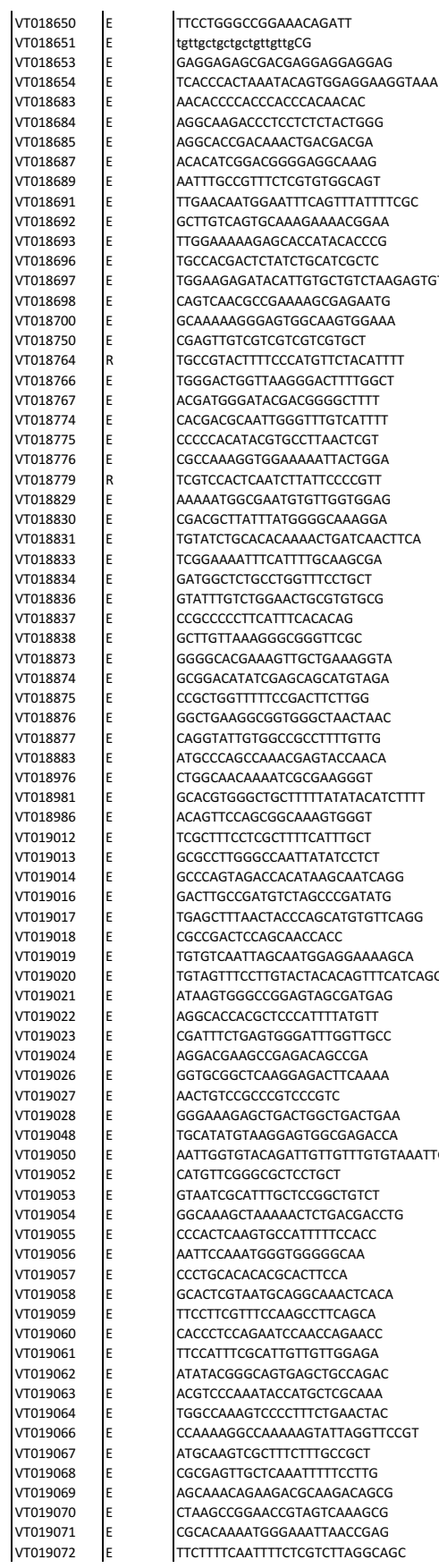
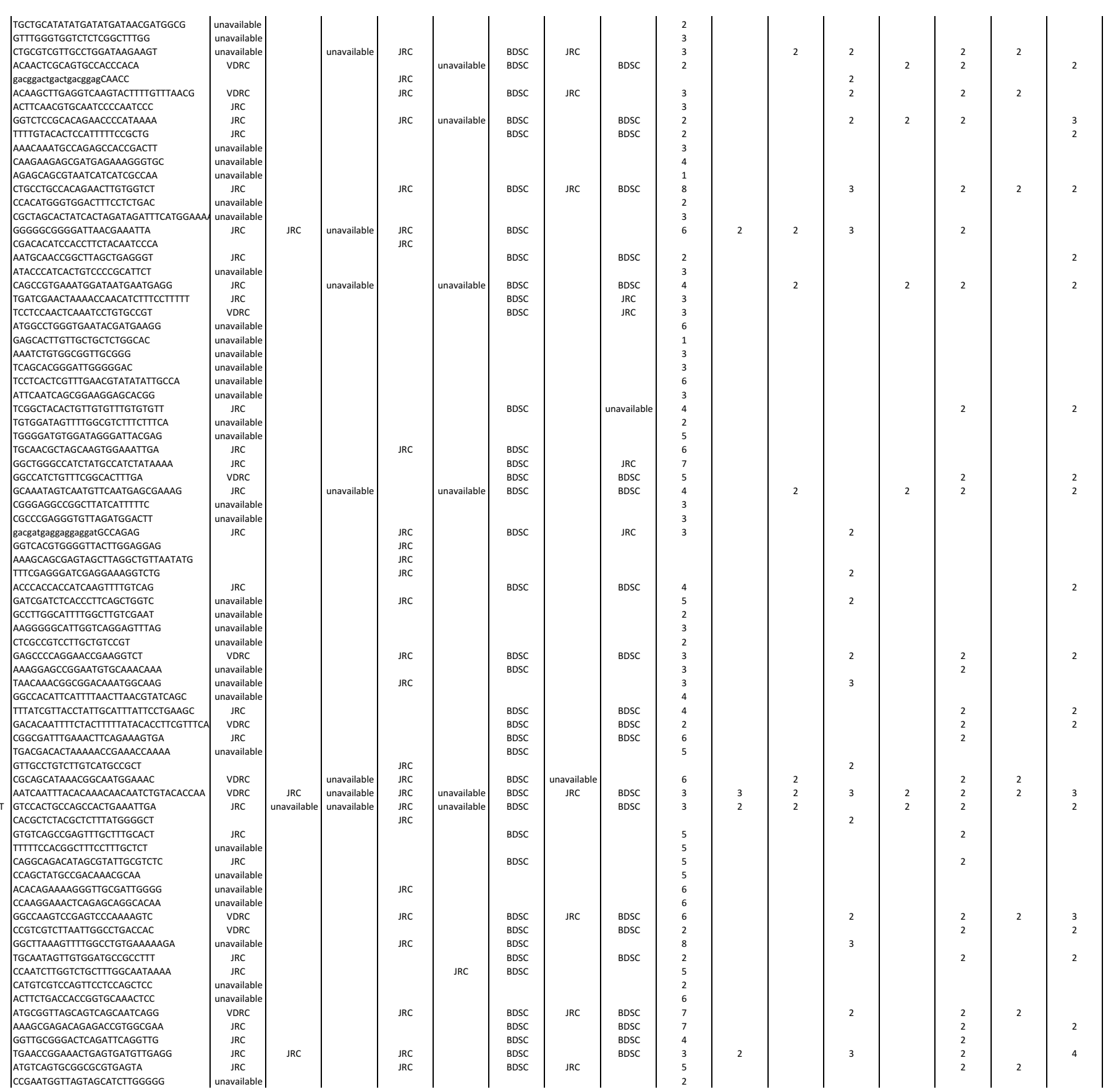


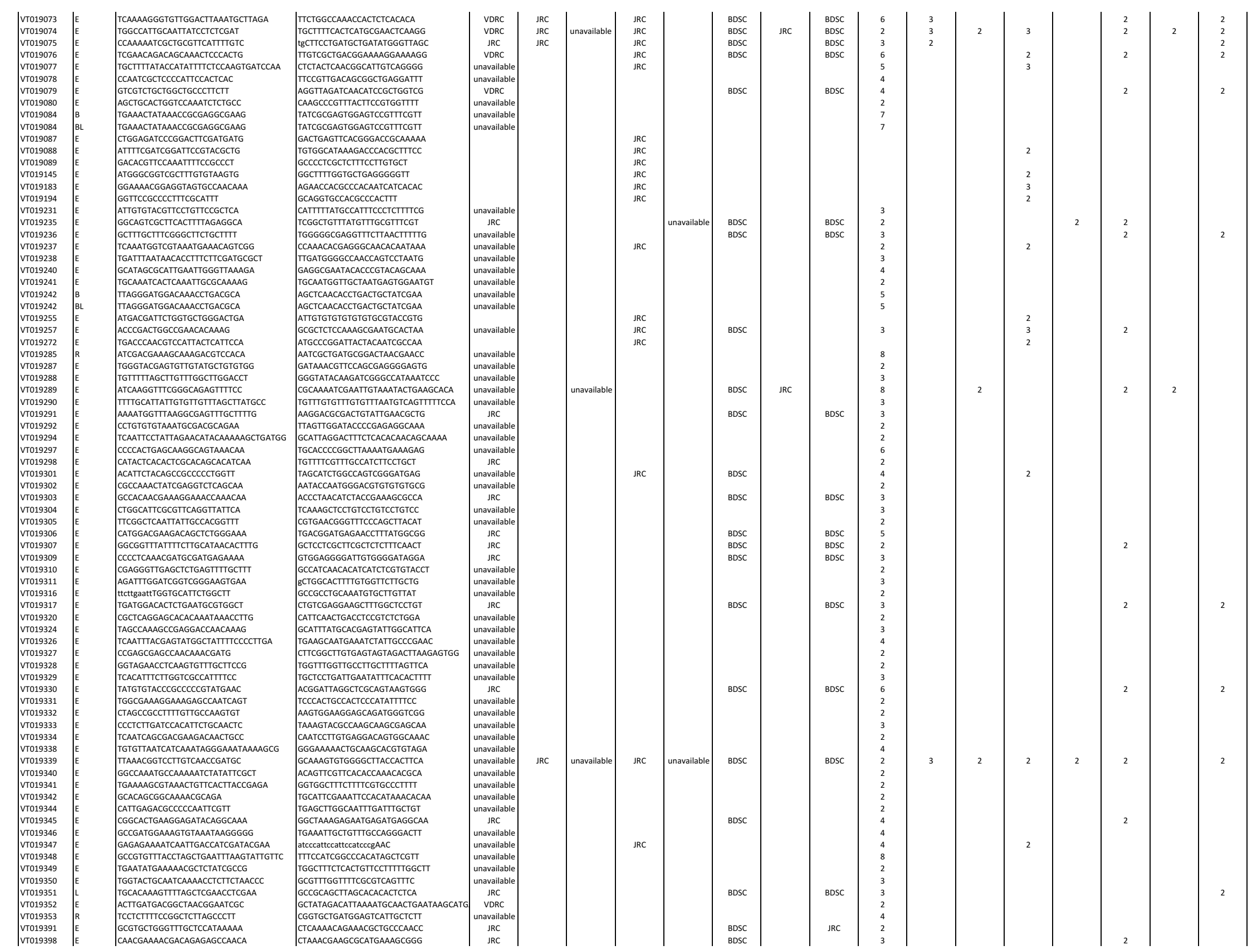




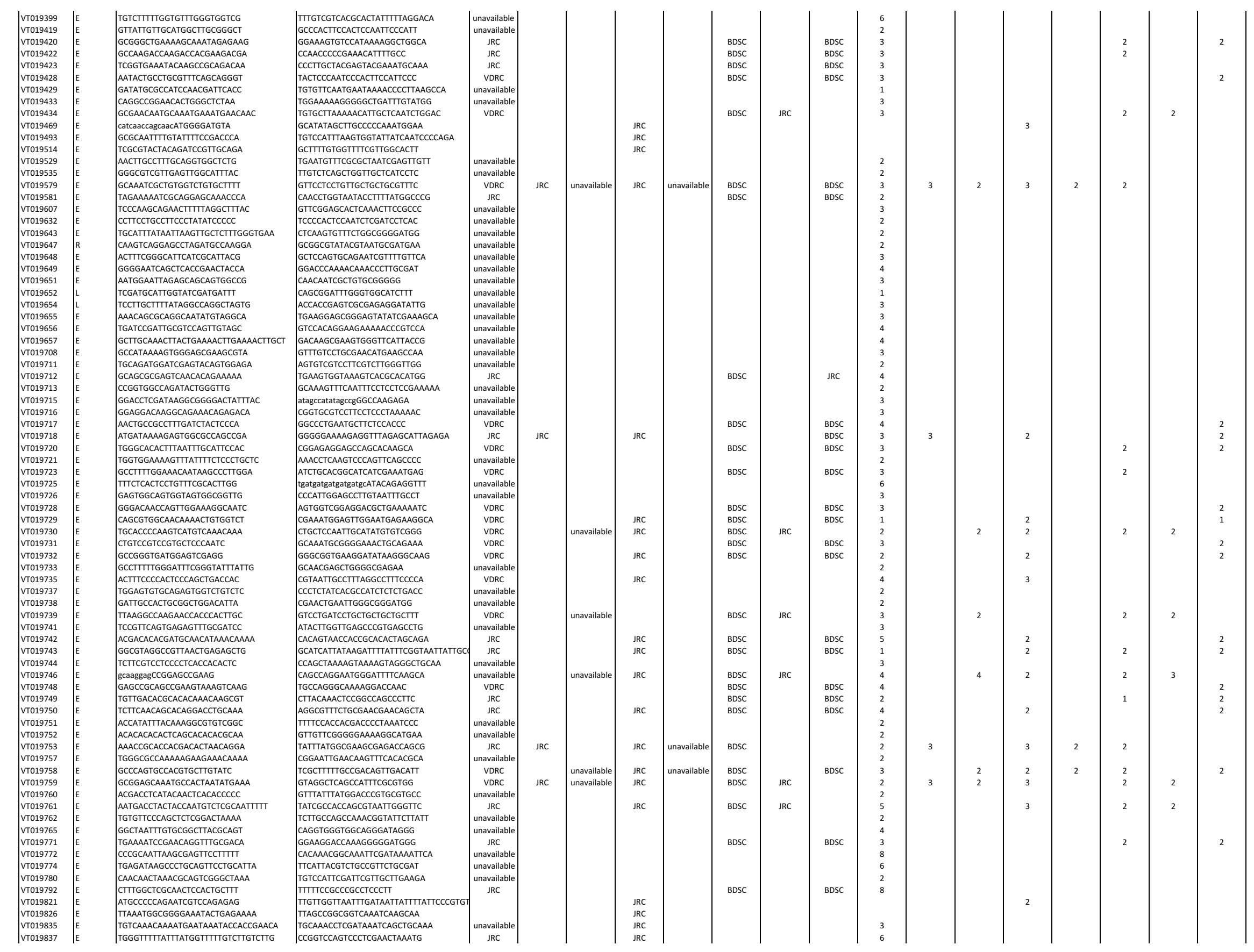




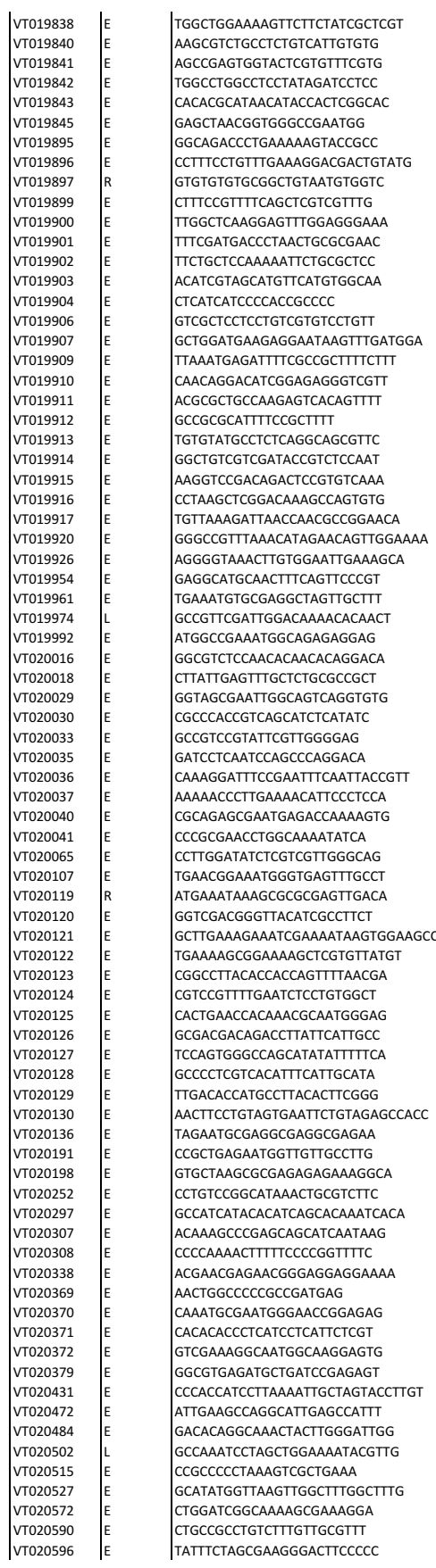

\begin{tabular}{|c|c|c|c|c|}
\hline 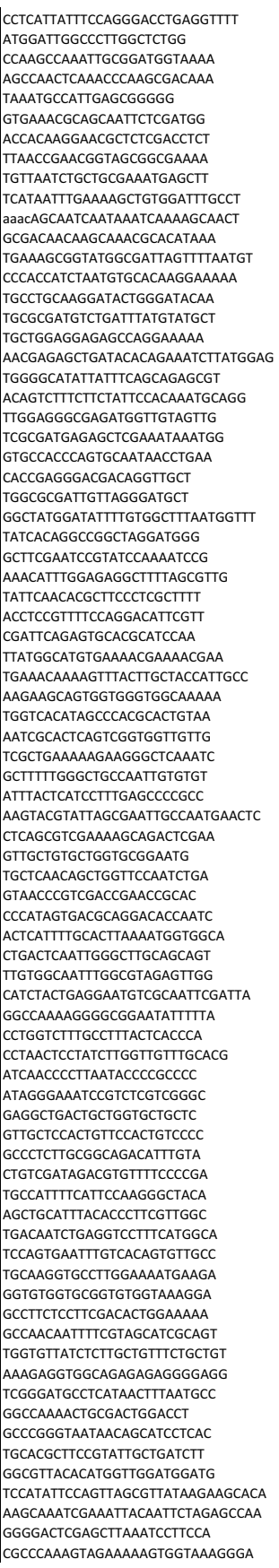 & 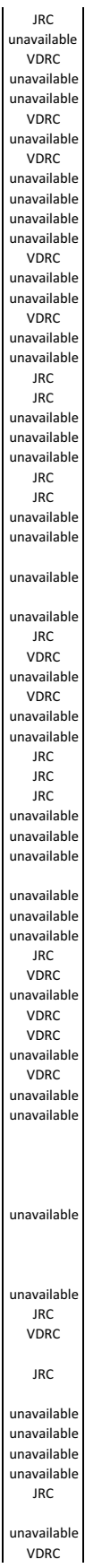 & $\begin{array}{l}\text { JRC } \\
\text { JRC }\end{array}$ & $\begin{array}{c}\text { unavaliable } \\
\text { unavaliable } \\
\text { unavailable }\end{array}$ & $\begin{array}{l}\mathrm{JRC} \\
\mathrm{JRC} \\
\mathrm{J} \mathrm{RC} \\
\mathrm{JRC} \\
\mathrm{J} \mathrm{RC} \\
\mathrm{J} \mathrm{RC} \\
\mathrm{JRC} \\
\mathrm{JRC}\end{array}$ \\
\hline
\end{tabular}
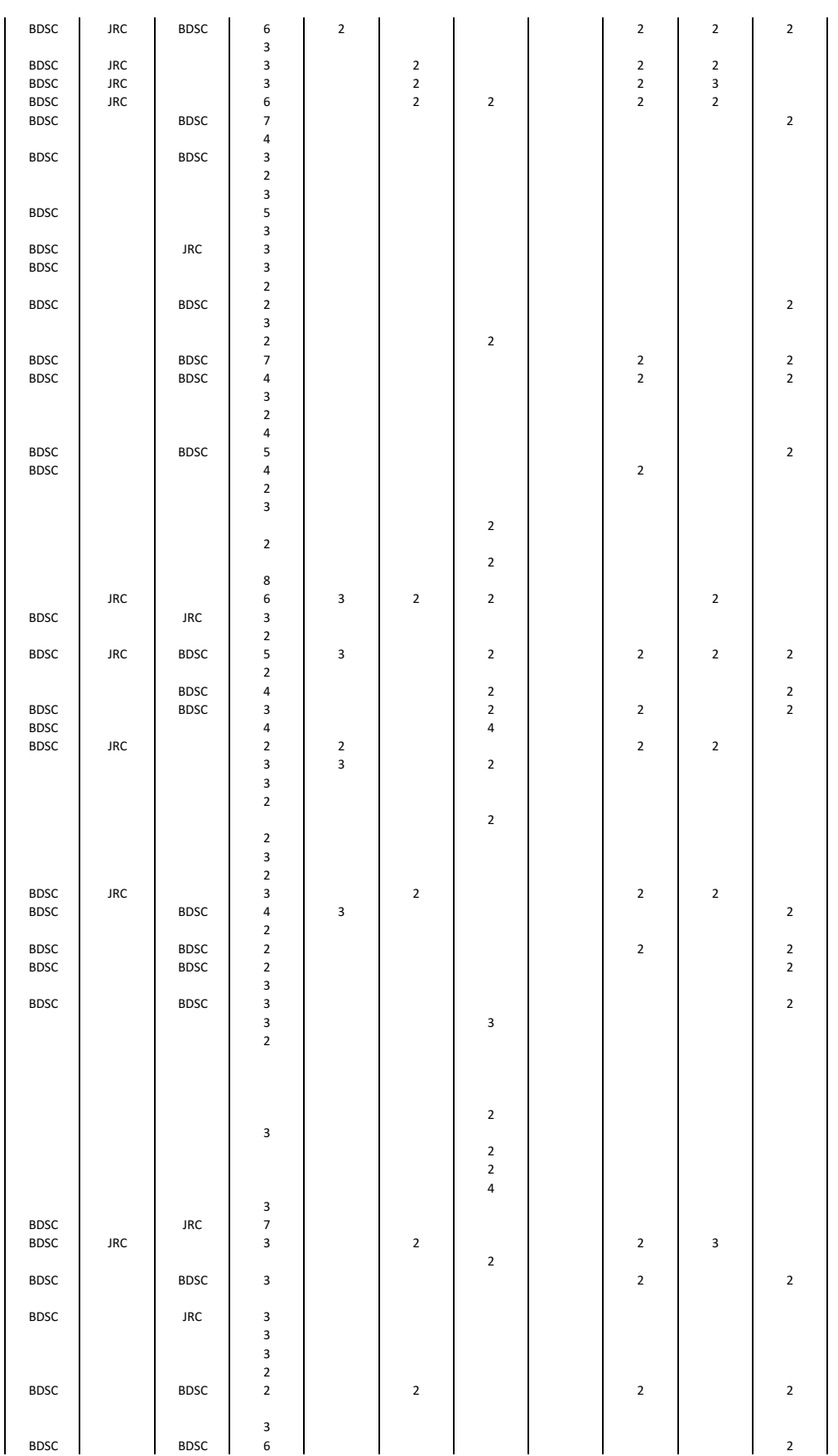


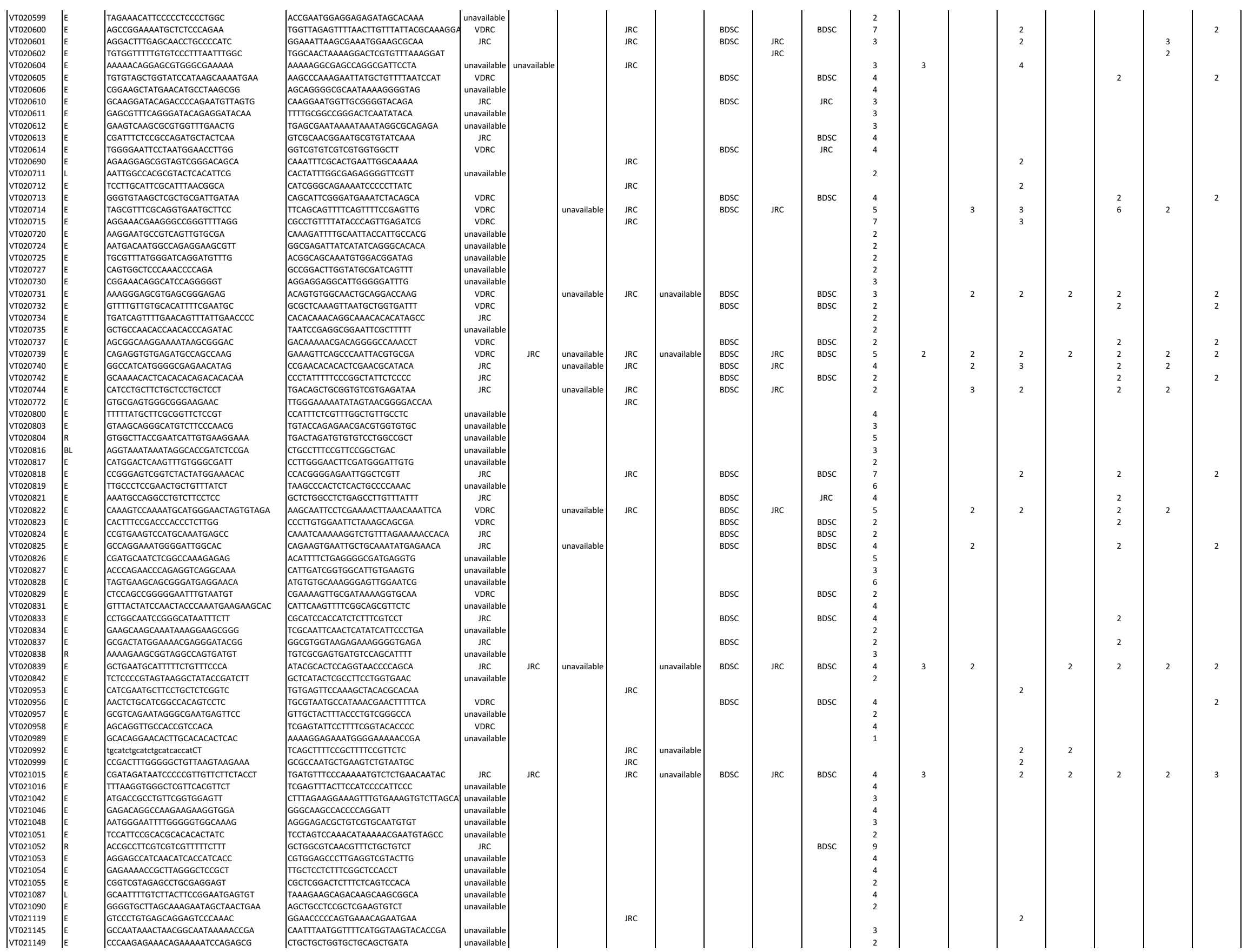




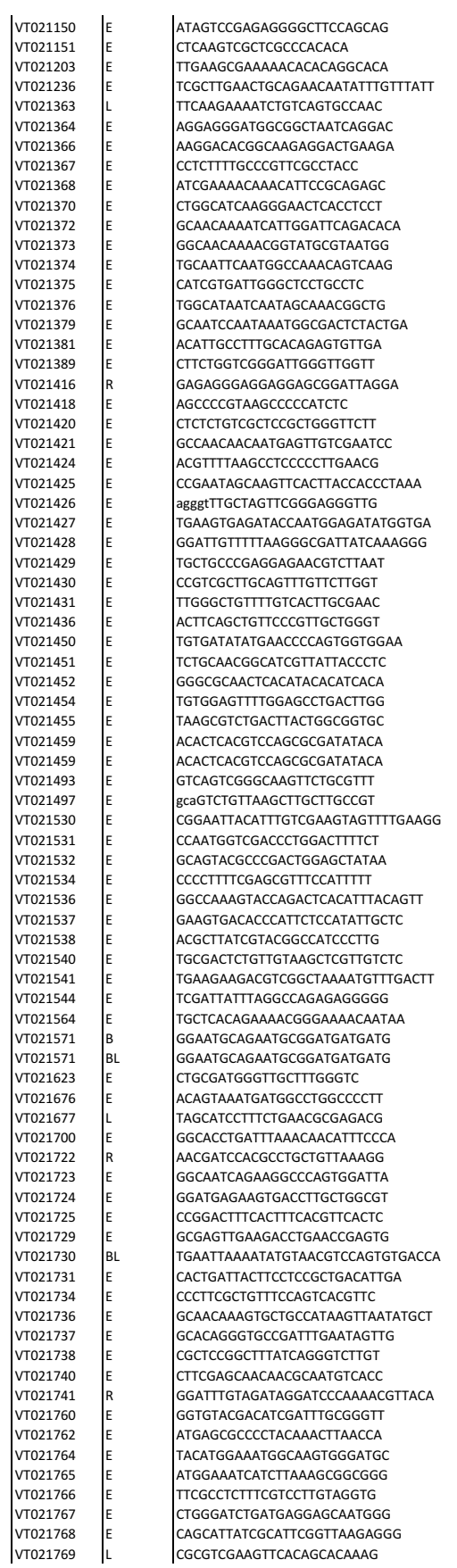

GGACCTGAGGATCCAAGTGGAGAA CAGGCAATTACTTCGAGTCGCATACC

TGGGGATGATCTACAATAAAGTGGAA GCAGAGAACAGGCAACCGGAAACTC

AACTCCACCATGGACAGCACGGA
GCCTGCTAACAATTGCAGCTCATC

TCTGTCCAAGGAGATTTCCAGTCCA

CGACTTTAAGACAAGAACAGCCATCGT

GATGCAGAGCACGTGCAACAAACAC

ATGAAAAGGGTGCACTAAGCGGTG

CGGCCAATAAATCTGCCACACA

ATTGGCCGCAACTATGGAAATGGA

GTGTCCTGGCGGCAGCTTGTACT

CTTCAAGGTGAAAGTGGACCCT

СCCTCCCCCTCCCTCATCACATAAA

GCCTACTGGATAACACCCCTI

CCTTCCATCCTGCTGGCTCCTITTG

TCCTCGGATAAAACAACAAATATCACAAATC

GGCACTTGGAATTAGGTAGTCAGTAGCG

ITCGGGCGGGAGAATATAAGCAG

gtatctgtatctgtgcacgtaaGTATGCGAG

GCTCTCGATCTGACTCTGACTCTGGCT

GAGAaCTGACTGAaGAATTGCTGCG

TCTGGTTACACTGCGCCTGGAAA
CTAGACACCGGCACAGTTGGCAG

GGCCACTAATGATTCGATGTGTGTTT

GGCGGAAATGGGAAGGAATAAATAAA
TITCATGGGGTITGGCCGTIC

ПTTCATGGGG TITGTGCCGTTC

TGGCATACATAATTAGTTTGCTATCAAGGG

IGCACCTCGAGATCTGACGATCGAA

IGCCGAATTGCTGTTAGGGTGTIC

TGTCTICGATTCAAAGCGGTGGTG

ACGGCCTTGACCTCCAGGAGATTG

ITCGATCCGCCATATTCACCCAA

TACATGGTGGCAAAGTGGGTGA

TCGGITGGCGTTGATTGCTT

GCGAGTGTCTGTGCCCCGATTCT

gtaCAAagtaTAaGCCAAAAAGAAAATGg

TGAAATTCTGACGCACTTACGGATTG

GAAAATTCTGAAGCACTTACGGATTG
ATTACTTGCATACGGGGAGGGG

G GGTCGTTAATTT

ATATAGGGCTGGACAAAACGCCGAA

ATCCACTGGGCCTTCTGATTGCCA

CCCAAGTCACGCATCCCATACAAAC

SСTTCCTCCATGCCTTGACCC

ACATGGGACCAACGTITTGGGTT

CGTAATCATCGTGGAAGGGCTTTGG

GATCGGCCGCCTTTCGGG

CAGGTGGCCGTGGTTCTGATG

AATCATGCCAGGACGATCGGG

GITGTCAGCACAGCCGGATTCTT

СCTCCAGATGACTCACTTGGGGT

GGACCTTCGAGTGGAGTTGCGTG

AGGAAATGAAGCACGAATGGGACG

СТAAGCTGCGGGCTCCTCTCTGTCT

ATTCCGGTCTCTGGTCGTCGTG

AGCGCAAGGGAGTCGCCACA
GCCGCCTCAATAGTACCAGCTACCA
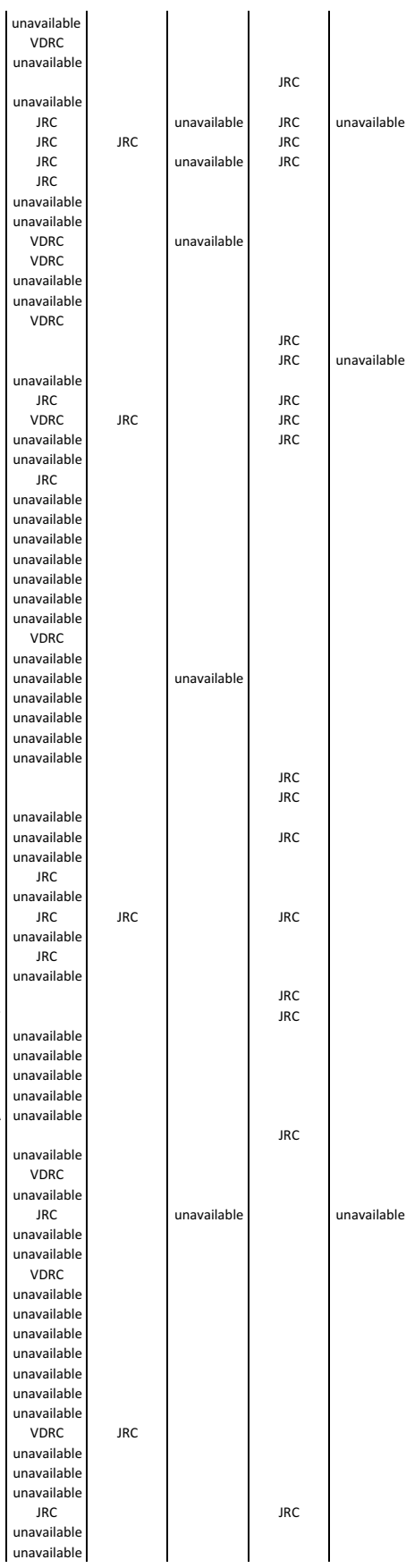

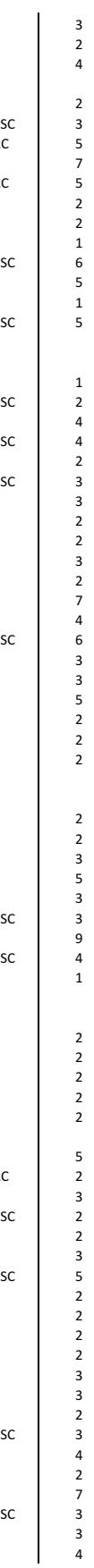
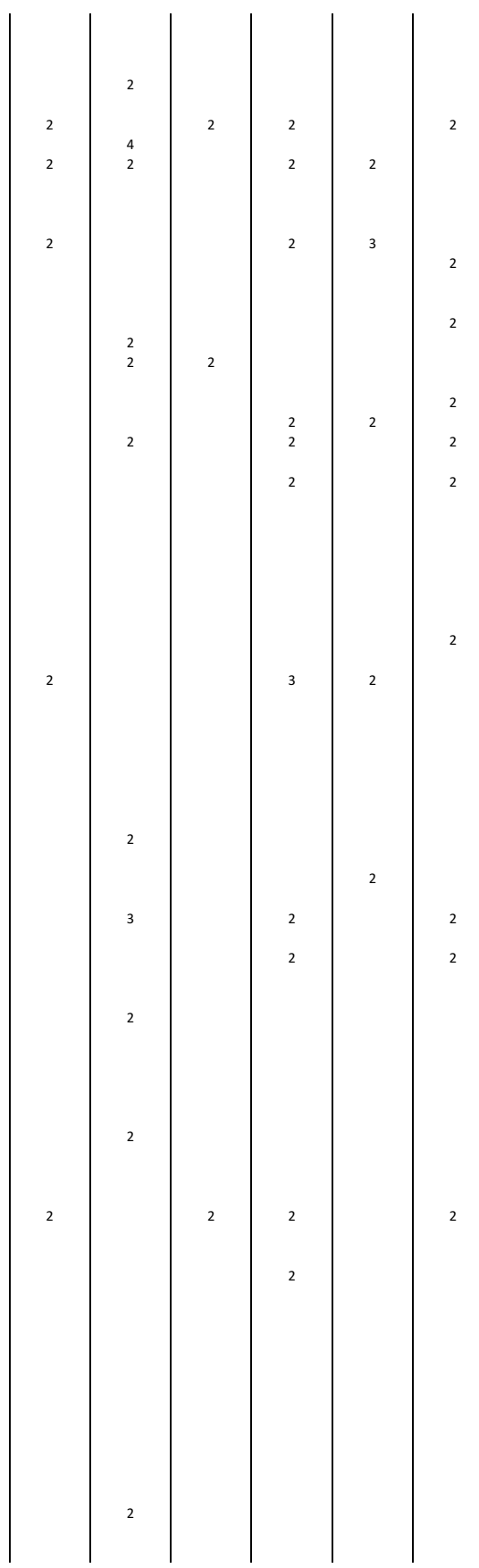


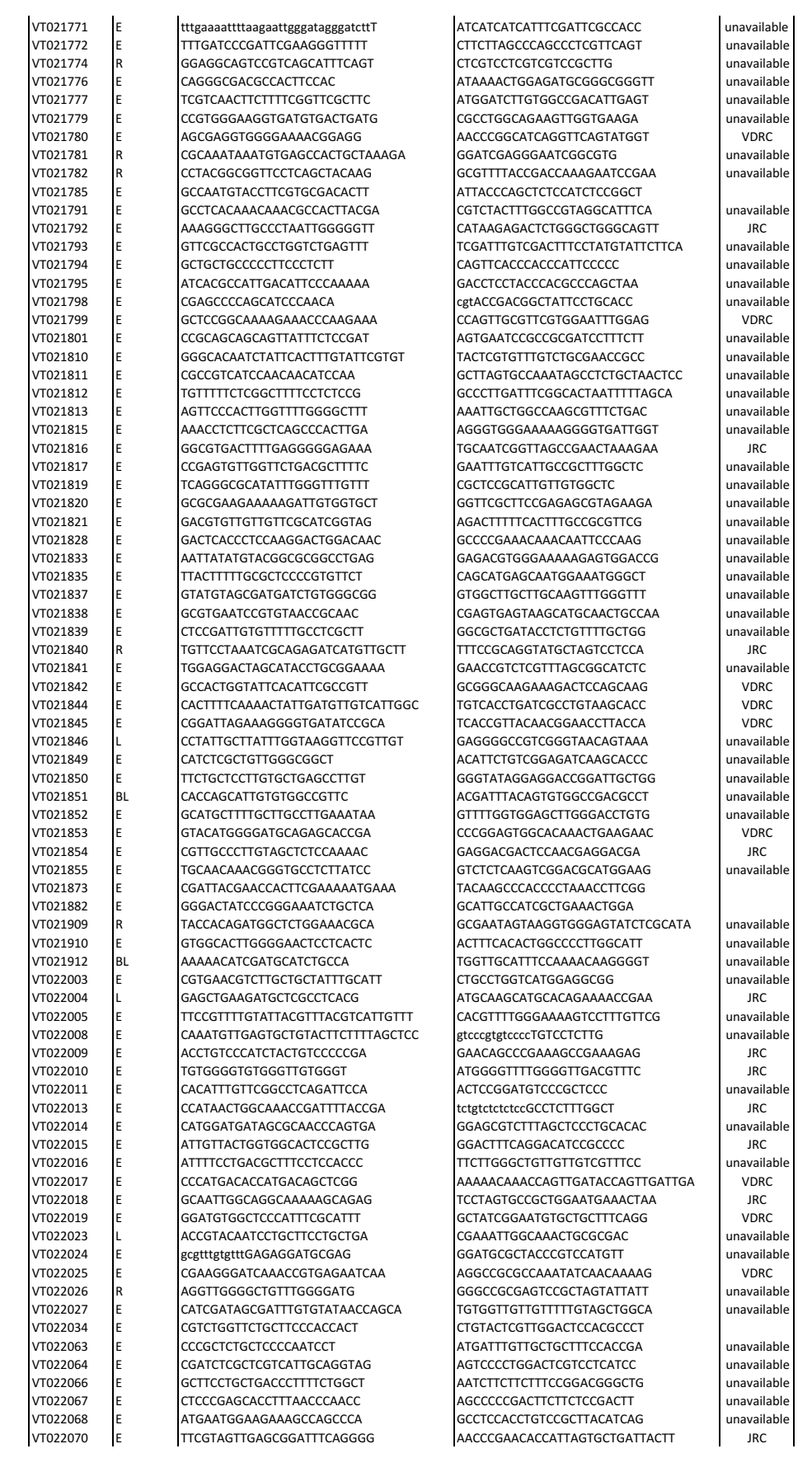

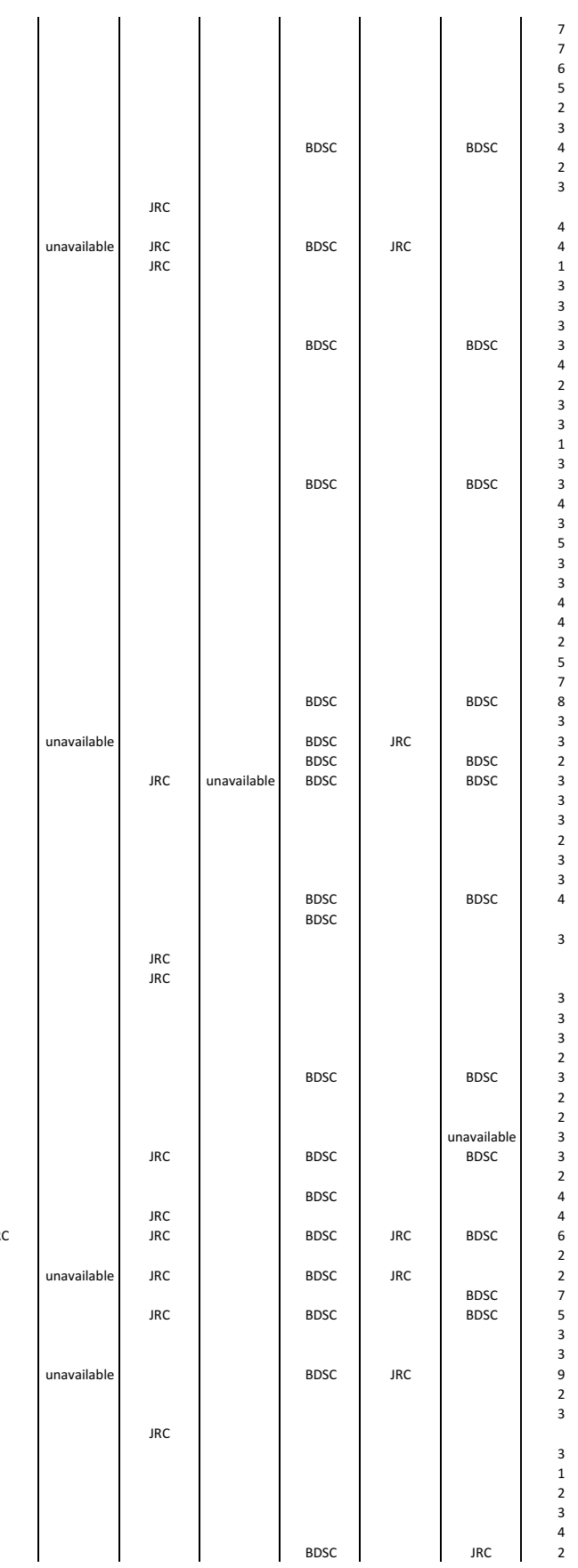




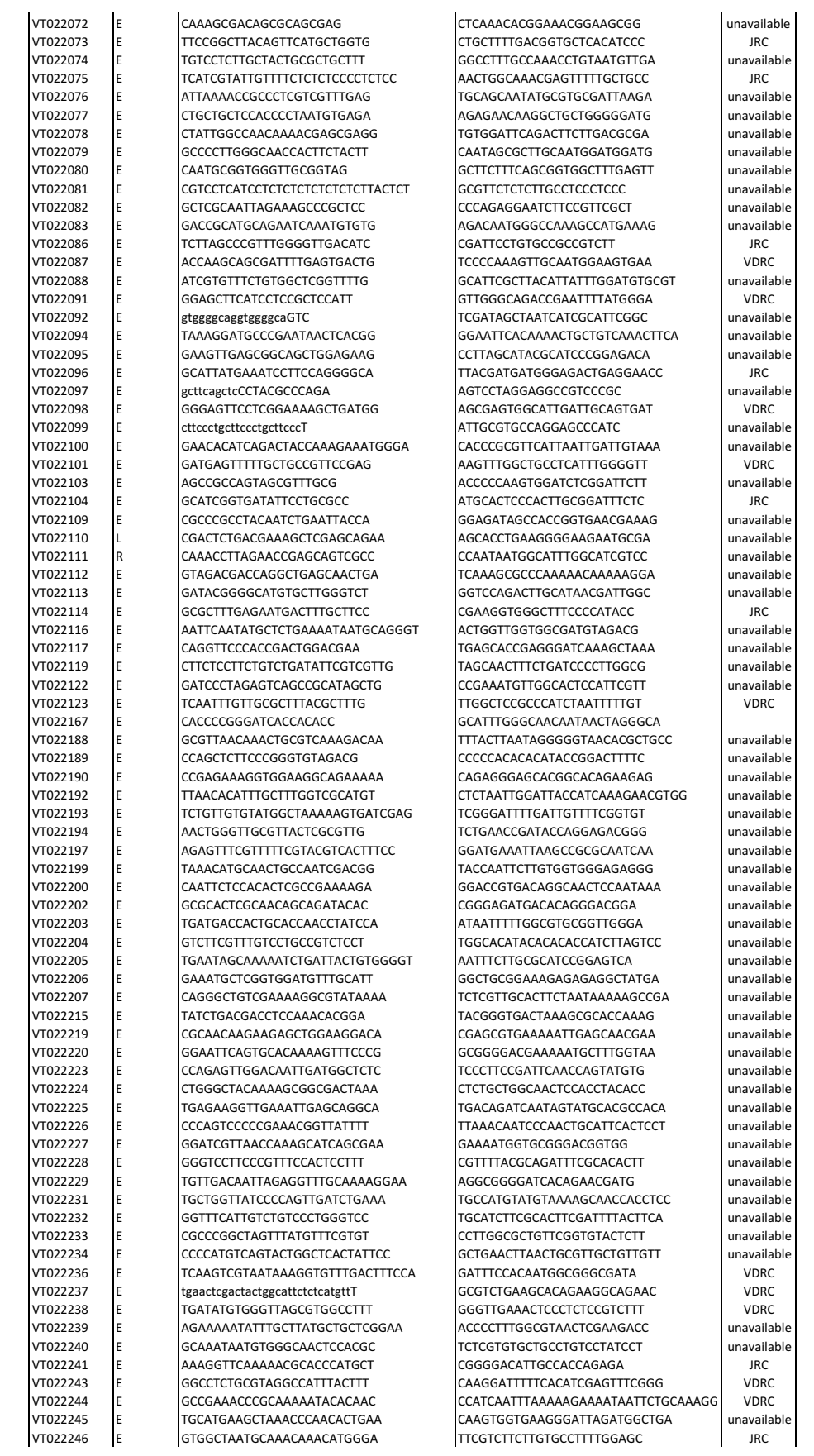
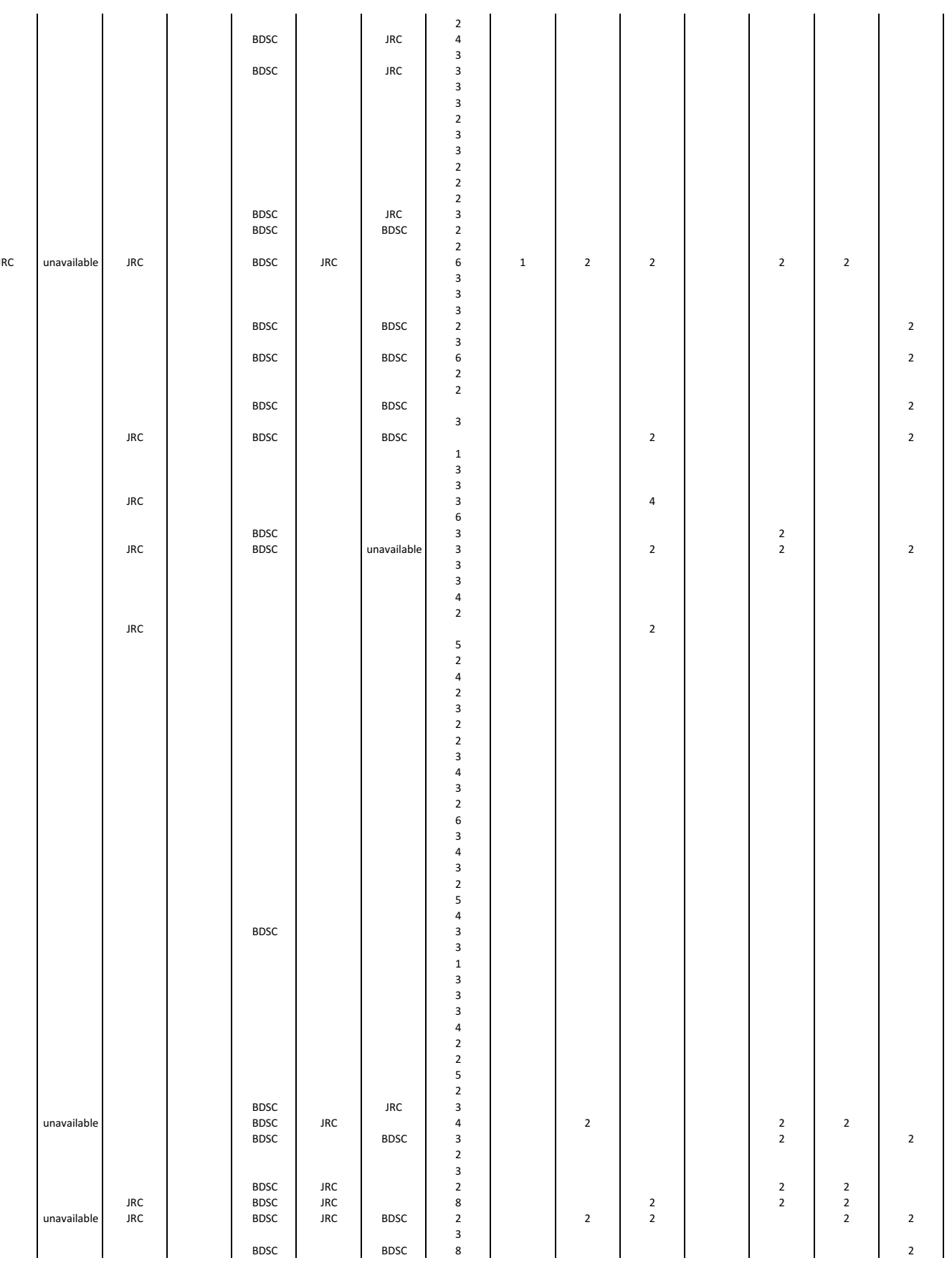


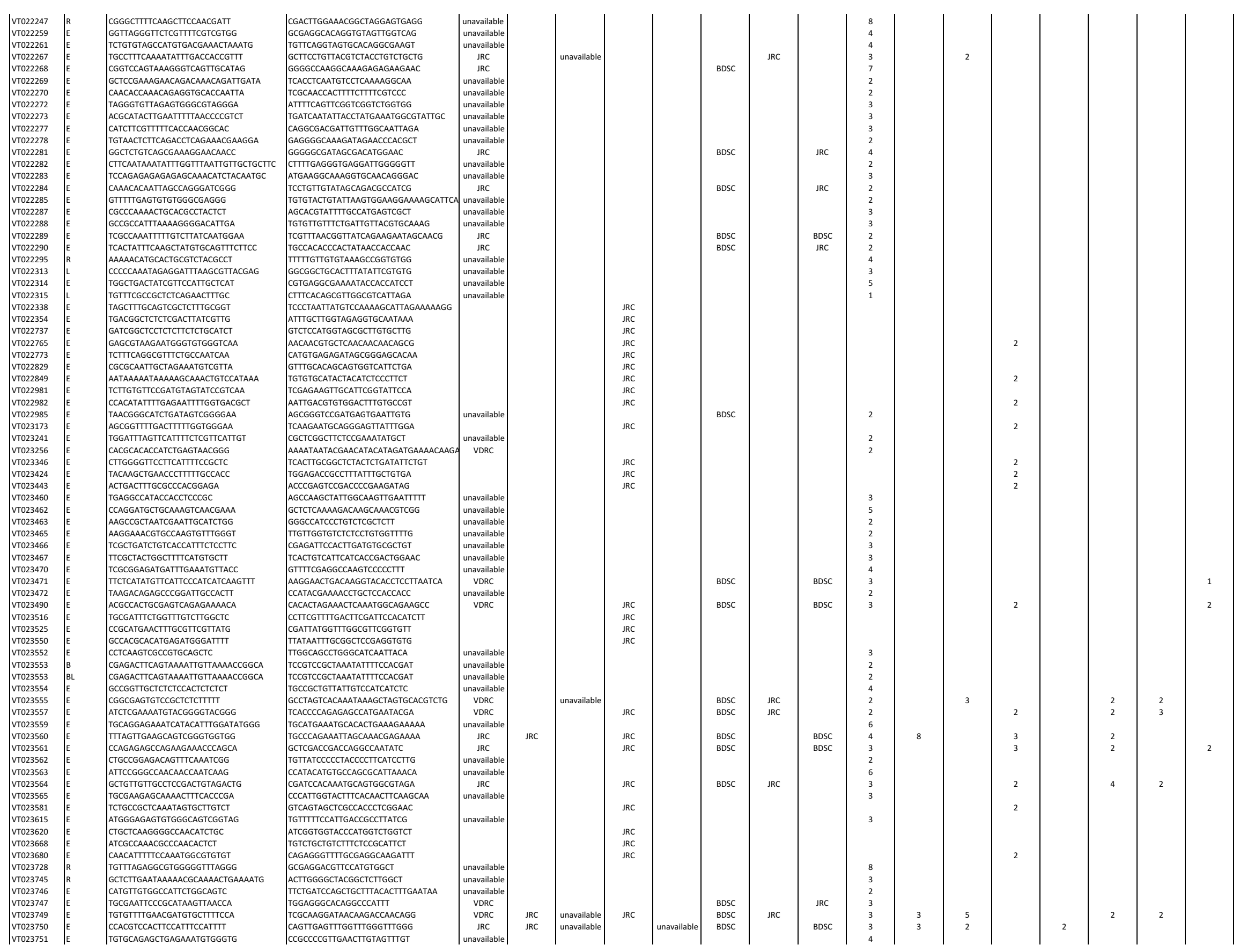




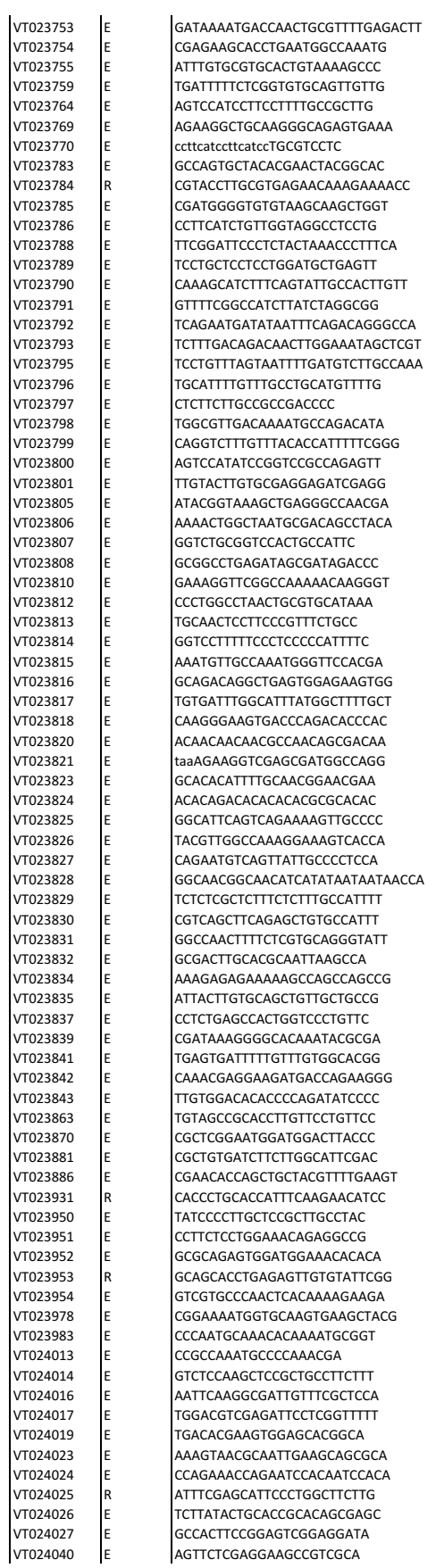
GTCTCGGGGTGGTGGTCCAAAG GTGGGCTGTGACGATTCGAAGG CAAATAGTCAATTATGGTGGGAGCGA
IAGCGAACATTCGACACATGGG GGGGGGATGGCCTGATCACGTAC ACCAGCTTGCTACACACCCCATC GACGTGCTCGGCACTCTGGTACTTC GTGCCCCGTACTTCCTACTTCCCT

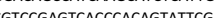
CTGGGTGGTAGCGGACTACATTGCC GCGTCTTGAAGGTCTGTIGTCAA AAATCGCAGGCAACGA CCTGGTTCCGGCAGTCCAGTGT GCTAGCCAGCAGGCAGGATTAAAACA GGATTTCCATCGCCTGCTGCCT GCCAGATCATGGGATGGCTCCACG GATGATGATGGCGACCCGAGATTG AGCGTCCAAGTCACGTCCAAAAC GGACACCAAGGACATCAAGGAGAG GGGATAATCGGGGAGTTGAAAAGGA GCCAGCAGAAGCAACAATAGCGAGA
GCCTTGAGCGGGACAGGATGT AAAACTCGCACTTGGTGAAGCAGCC GAGAGAAACACTTTTACTTGCTGCCG GTCGGCTTCCTTGCGTTCGT

GGGCAGCAGCAGACGGATTIT GCATTTGGCCATAATTTTTGACGG CACTTGTGAGGGGAGAAGACGCACT GCATTTTACACCAACCCCTCCGAA

TCCCTACTATTACGAGCAAATACAGTTGCAA CGCTAAGATTCTAAACAGCTGGCTCGC ACTACCACAATTAACTIGGGCCAATTIT CGATTGGGGCTAAAGACAGGAGGCA CCACTGTGCGCAGCCTTAATTCATTT TGTTAGCTAGCAGATGGACTCCGTATCC CCAAACAACCCGAAAGCCAAAAGAA AAGACTTTTGGCCCTGTTTGTTGGC GTCCTCGTCCTGGTGCTTATCCTT GGAGCATGGATGTTTAGGCAGGCA
GCGATCGGGAAAGCGAAAAG cagcaacatcagcGTGCCCAAC
CCCCGCAAATATGGGGAT CGAGCAGGCCATAAACCCACATAGA GGTGGCCTCTGTGAACTACACTTCCG TGATCTTCATCTGCTCATTTGTTCTG AAGAAATCGTCCAACTGAAAGCCCA TCATTCCGAGCAACCCAATGTTTTC CTTAGGAGCTTGTCCTGGACGCCC ICAGTAGTAACGTTAGCTGAAATCTTCTTT agGGTGATCATCGGGAGCAA GAGGGGTCTATTTTGGGGGGTC GCCTACCTCCAACGAGCTTCACACA ATGTCAACTCGGTGCGATGATGATG GGCTGGGAAGGACTTGGAAGGGG TGCACAGATACAAAATGGTAACTGACTCAAA TACGCGGCAAAAGTGGACCTCATC CGGGTGGCACGCAACTGACTTT GCCCAAATCACCTTGGTTITGTTT GACGATATTGAAGCCCAGGTGGAA
GCATTCGGCATCTGGTCACAC

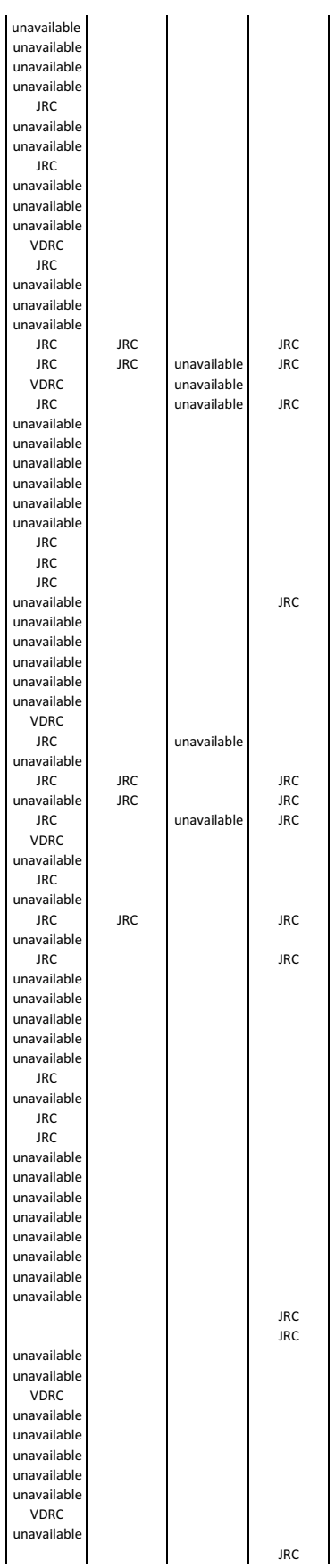

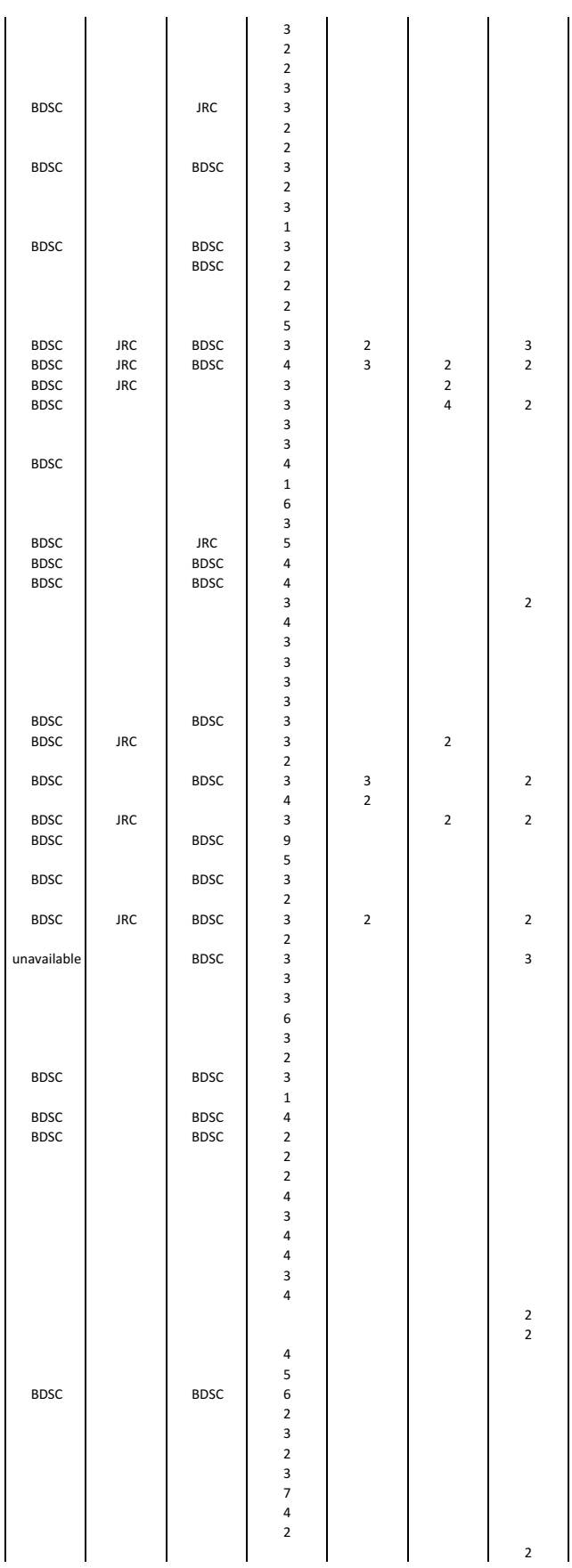

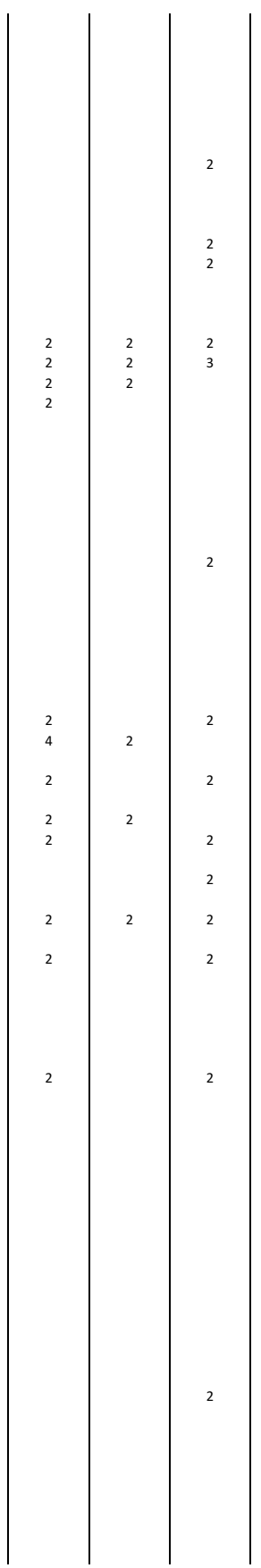




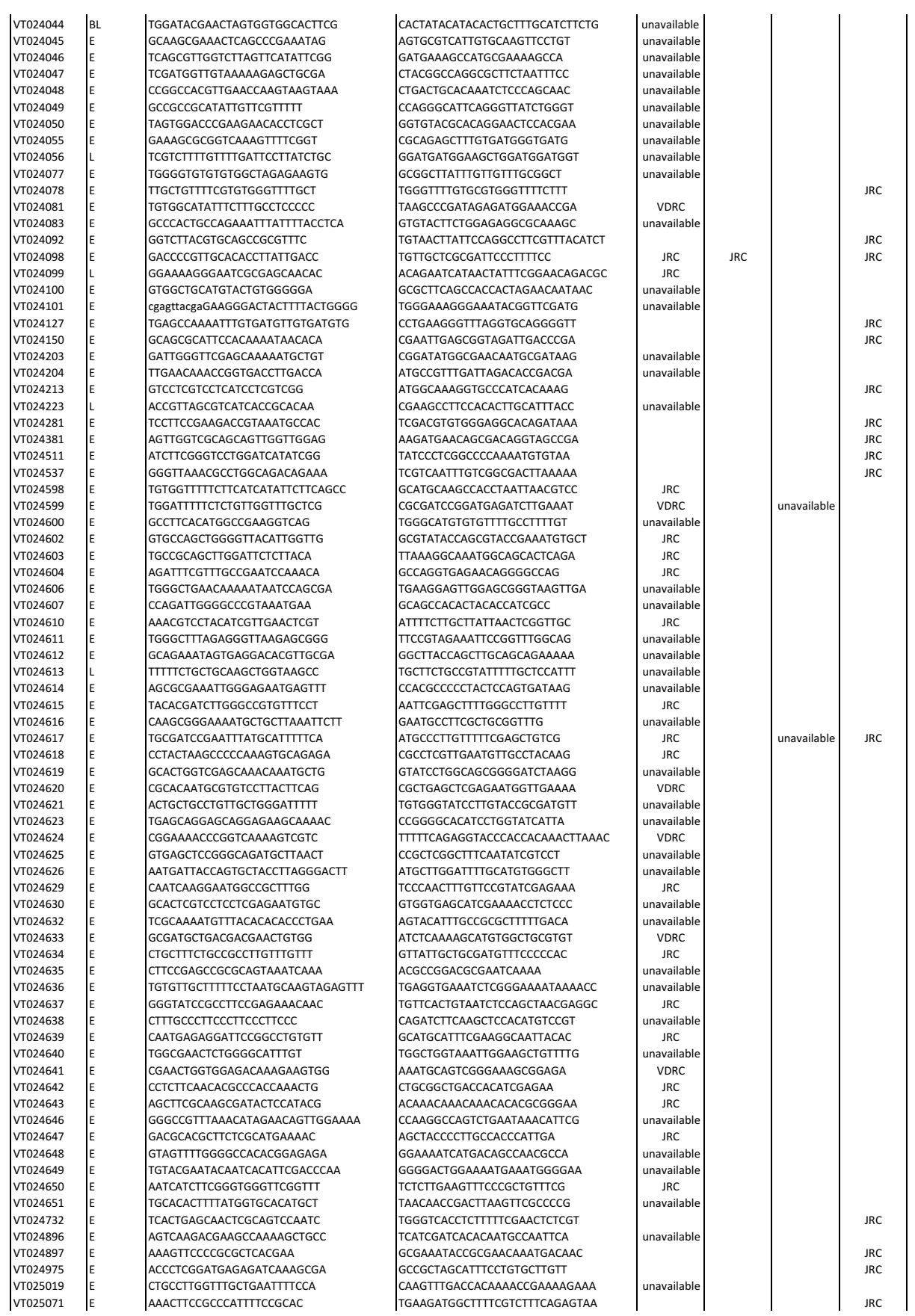
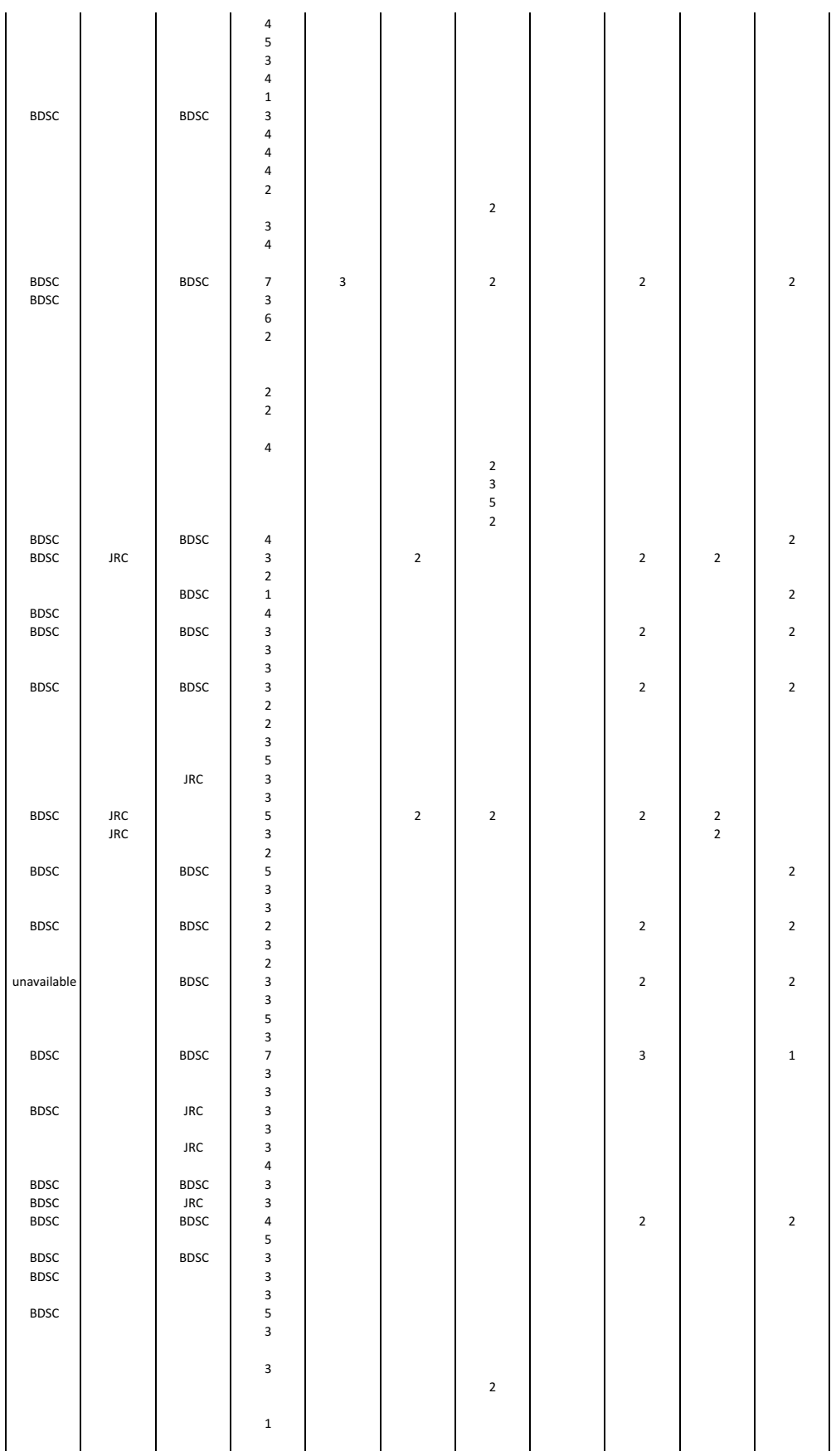


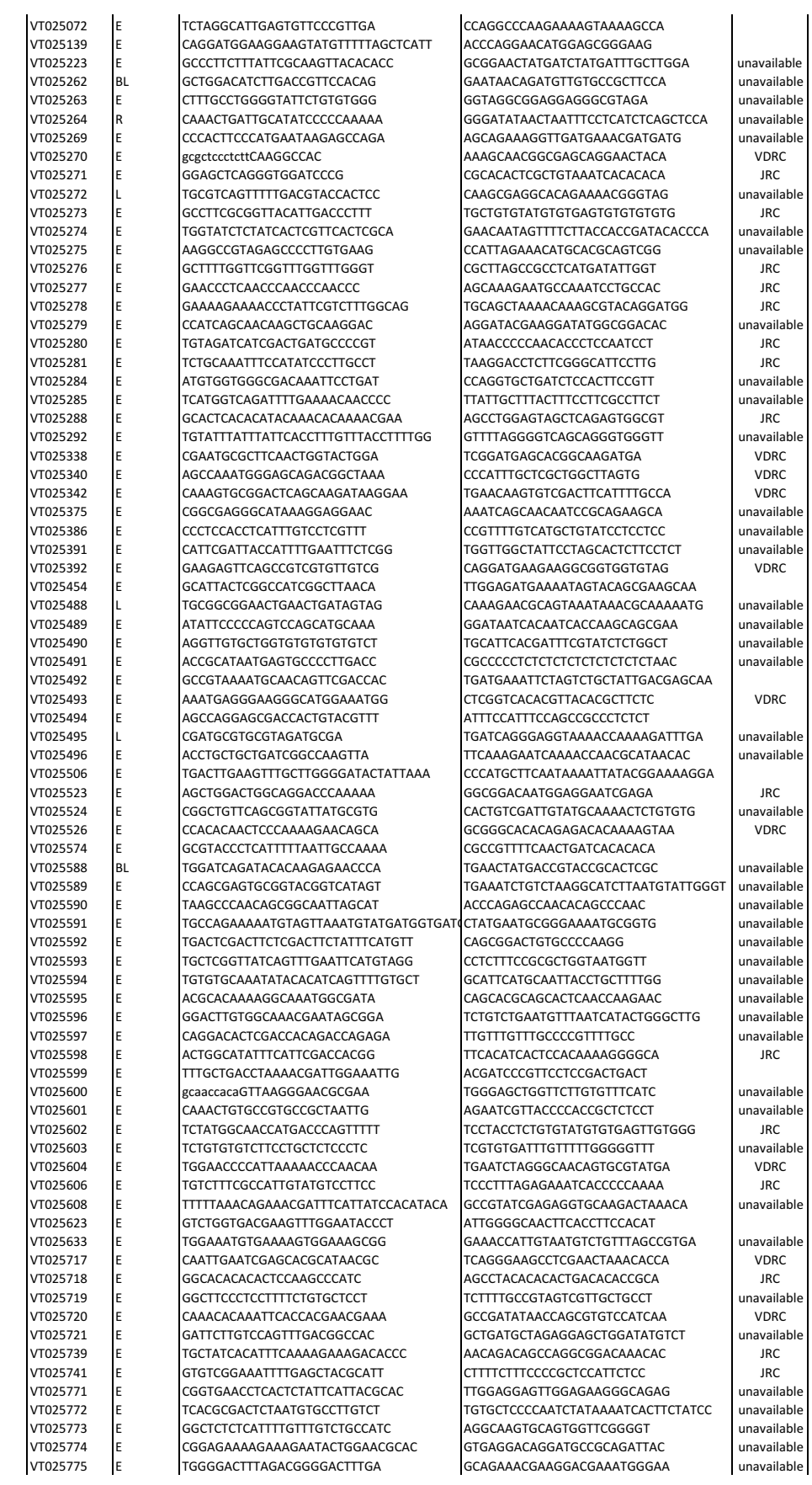
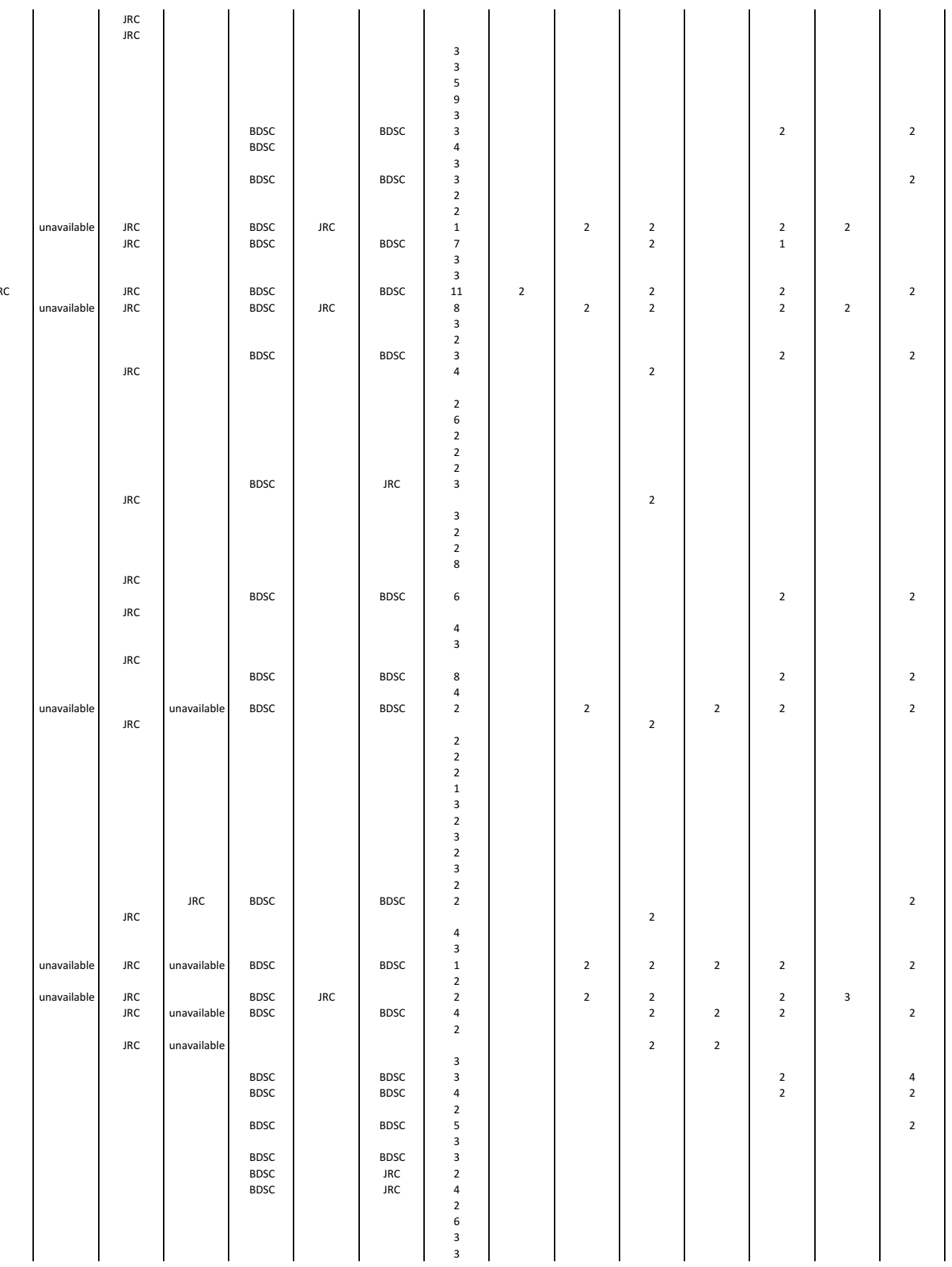


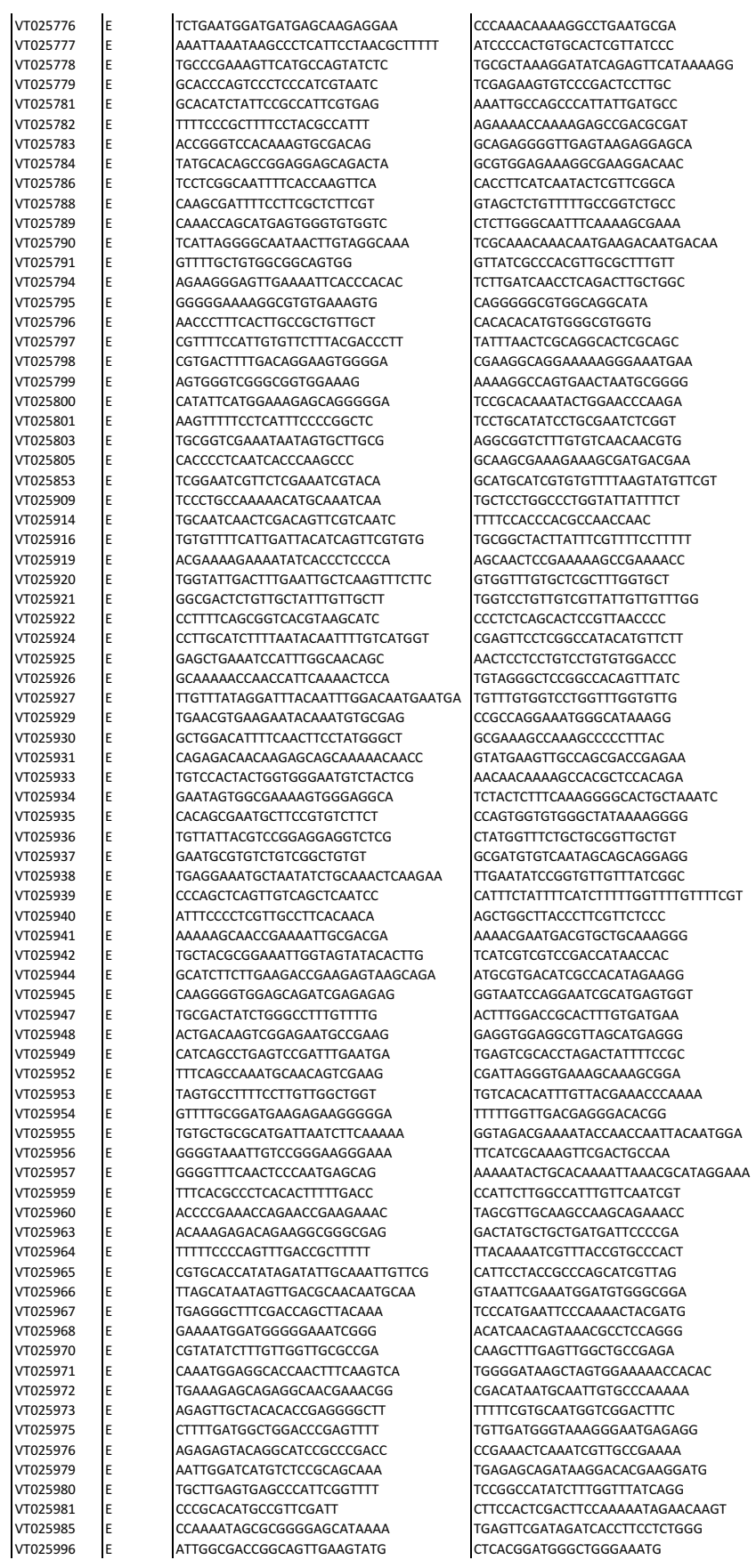
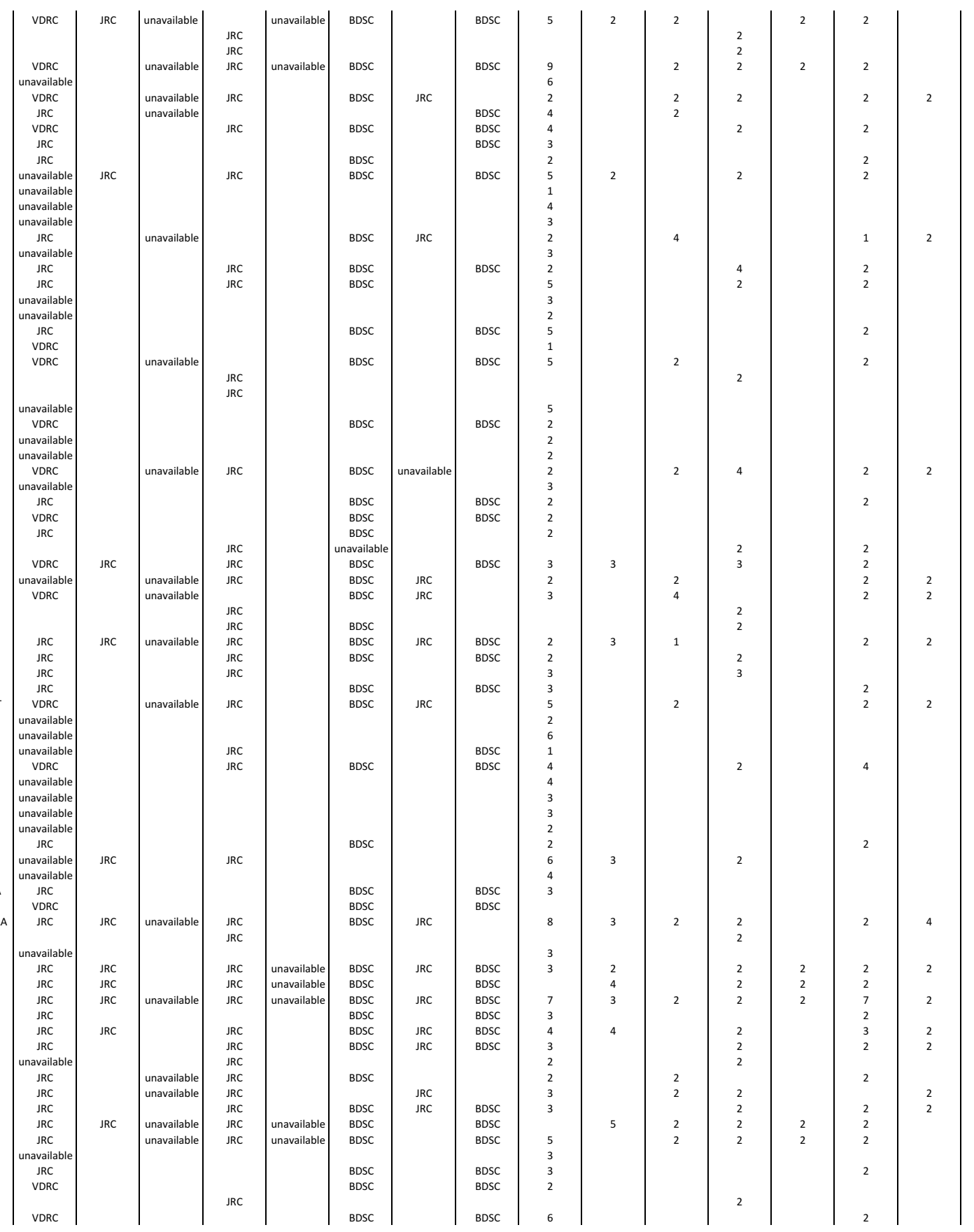


\begin{tabular}{|c|c|c|c|}
\hline 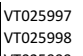 & $\begin{array}{l}E \\
E\end{array}$ & \begin{tabular}{|l} 
GAGTGCTAGGTGAACTCCACAACGCC \\
TCAGTTGCCGACAGTTTIACCCC
\end{tabular} & \begin{tabular}{|l} 
GCAACAAATGGAACAAGACAGCAATGGA \\
CTITICCCAGCTCGCCCATTGACTT
\end{tabular} \\
\hline $\begin{array}{l}\text { VTO252599 } \\
\text { vTro2600 }\end{array}$ & E & GCCAAAGGCATATTAAAATCCATTTAGTTCCA & сCTIGTGGGATCATTTTAGGGCCA \\
\hline $\begin{array}{l}\text { VTT226000 } \\
\text { vTO26001 }\end{array}$ & ${ }_{E}^{E}$ & $\begin{array}{l}\text { TTTGGGAAAAAGACGGGGATAATTTCTCG } \\
\text { TGCAGGTTCACTTTCCACGCT }\end{array}$ & $\begin{array}{l}\text { GCGGCCCTTAGGCATTCTITTCCT } \\
\text { CCATAAAACTCGAGCGACTGGTGC }\end{array}$ \\
\hline VTo26002 & $\mathrm{E}_{\mathrm{E}}^{\mathrm{E}}$ & GTGCTCGGCCAATGTCCGTCTC & GACCCAACCGACCGGGCAAC \\
\hline vT026004 & E & TGATGACTGCGITTGAGATCGATGAA & ACACCACCGCGTTACTTGGTTTT \\
\hline $\begin{array}{l}\text { vTo26005 } \\
\text { vTro26006 }\end{array}$ & ${ }_{\mathrm{E}}^{\mathrm{E}}$ & |AAAGTTGGTAAAGCTGTTTTGCATTTTAG & TGGACAGGTGTAGTGCCAAGCACAA \\
\hline $\begin{array}{l}\text { Vit0260606 } \\
\text { VTO26007 }\end{array}$ & E & $\begin{array}{l}\text { CCCCAAAAACCAAACAACGATACGA } \\
\text { ATACGGGGTATGCTGCTGTGGTTT }\end{array}$ & $\begin{array}{l}\text { GCCCAGGCAAAGACAAAGAGAAA } \\
\text { CGTTCATTTGCCAATCTTTTGCCT }\end{array}$ \\
\hline VTT26008 & E & АСGGCTTACCCTCTTTACTTTTGTATCGAA & AAGATGGAAAGCAGCAAGGGAAGG \\
\hline VTo26009 & E & |TCTGATCGCTAATTGGGGITITC & TCAACATCATTCACGCCTCACACAA \\
\hline $\begin{array}{l}\text { vTT226010 } \\
\text { vTO26011 }\end{array}$ & ${ }_{E}^{E}$ & $\begin{array}{l}\text { GATTGCATGGGCCACAGATTTGGT } \\
\text { TITCTTTAGGCACACACATCCGG }\end{array}$ & $\begin{array}{l}\text { GCTCGACGAAGACCDTITCATT } \\
\text { TGGGGTIAAAGAAAGGTTACTCA }\end{array}$ \\
\hline vTo26012 & E & AATTGGAGTGGAATCGAAAGCCCAG & 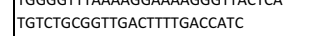 \\
\hline $\begin{array}{l}\text { VTT226013 } \\
\text { vTo26015 }\end{array}$ & E & CGGCACTTAAAGACTGATAGTTTTTCCCGA & $\begin{array}{l}\text { GCTCGCTCAATCAACTATTCCAAAGCC } \\
\text { GCCCATAAGCAAGGCCCAGDTGTA }\end{array}$ \\
\hline $\begin{array}{l}\text { Vit0220615 } \\
\text { VTO26016 }\end{array}$ & $E_{E}^{E}$ & $\begin{array}{l}\text { GGGAGAGACACCGAAAAGCTCGTTG } \\
\text { AACGAGCTACGGAAAGGTAGCAGTTAAAA }\end{array}$ & $\mid \begin{array}{l}\text { GGCCCTAAGTCAGGGCCCAGGTTTGA } \\
\text { GAAGCAGGCAGATCTACTTACTACAGC }\end{array}$ \\
\hline VTo26017 & E & TTTGTCCCTCACACATCCAAGCACA & CCCGAGTGTGTCCCACTTATGCAGAC \\
\hline VTo26018 & E & TCACGTTATACCGCCTGAAGGTGATT & GCAAAAGCAAATAAACCAATCTACTAAGCGA \\
\hline $\begin{array}{l}\text { VTo26019 } \\
\text { VTT202020 }\end{array}$ & $E_{E}$ & |'GGGGAAGTGATGGTTGGAAGTGATT & $\begin{array}{l}\text { GCTTTTCAGAACAGGGCTCGAAGG } \\
\text { TitCAGAAAGTGGCATGGGCA }\end{array}$ \\
\hline $\begin{array}{l}\text { VIT12206020 } \\
\text { vTO26022 }\end{array}$ & $E_{E}^{E}$ & $\mid \begin{array}{l}\text { AAGTTGCCACTTTAAGGACCCCGA } \\
\text { GCAAGCCATGATAATAAGGGAAAGGA }\end{array}$ & $\mid \begin{array}{l}\text { TTATCAAAAGTTGGGCACTAGGCA } \\
\text { TITAATTCAGACTGTICAAA }\end{array}$ \\
\hline & E & TCCAATGCCAATGAATGCCAACAG & $\begin{array}{l}\text { TGICAATITCAGACACTCIICAGA } \\
\text { CGTTGTTCCAAGCGAGAAAAAT }\end{array}$ \\
\hline & E & GTGCATCAGACAGGAGTTGAGGGGA & GAGACTTCCATTCCCACAGACATTGC \\
\hline & E & CAGATCGGGGGAAGTGGAAGTAGGG & AАTTTATTGTCGCCTGCTGTTGCGA \\
\hline & E & CGAAGTCCAAAGCGAGTGAATACGA & TCCGTCTCTTTCTGCCCATTCAGG \\
\hline & E & TCAATGCATTTTCAGCTGCACTCTATG & GGAGAAAAATTCCCTTGGAAGGTTGTG \\
\hline vT026032 & ${ }_{E}^{E}$ & TTGTTAACCTAACGAGTTTCAGCCCC & AAGGGCGAACGATATTGCATTGCTC \\
\hline $\begin{array}{l}\text { VITT220633 } \\
\text { vTO26034 }\end{array}$ & $\mathrm{E}_{\mathrm{E}}^{\mathrm{E}}$ & $\begin{array}{l}\text { TCGGATGGAGATGAATGTGGGCAT } \\
\text { TCGCCAACACTGAGCTAGAAAG }\end{array}$ & $\mid \begin{array}{l}\text { GCTGGAGAAAGGGGAAAAACCCCC } \\
\text { CAGGTAGATAGATCAGGCGA }\end{array}$ \\
\hline 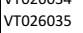 & E & $\begin{array}{l}\text { TTGCCAACCACTGAAGCTAGGAAAG } \\
\text { ttagtttataaaTGCGCCAAAGCAAGCC }\end{array}$ & $\begin{array}{l}\text { CAGGGGCAGATAGATCCAGAGCCGA } \\
\text { TGCCAACCTCTICATAGGAAACACATCC }\end{array}$ \\
\hline VTT226046 & E & CAGCATCCTTACGTGCTCCCATCCT & GGTCTGTCAAGTTTCGCCCACCTCT \\
\hline VTo26076 & E & сCTTGGATGATGTCAACAGGAGGGA & GTGGAATGAAAATCG TTGCAGCAC \\
\hline 6 & $\mathrm{E}_{\mathrm{E}}^{\mathrm{E}}$ & $\begin{array}{l}\text { AAACACACCAATCCGGTCACATACGCTG } \\
\text { CAGTTAGCGCAGCAAGACACAAGT }\end{array}$ & $\mid \begin{array}{l}\text { TCCGGTCACATTGTCAAAACAAAA } \\
\text { CAAGCAAGGAGCGGAAA }\end{array}$ \\
\hline 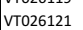 & E & 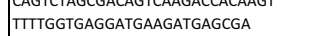 & $\begin{array}{l}\text { ACAAGCAAAGGCACGCCACGAA } \\
\text { CGACAACGAGGACAGCAACGAAG }\end{array}$ \\
\hline VTT26123 & E & CGGGGAGAGGAACCACATAGAGAGG & GCTGCAAGGATCGGGAAACGAGTAG \\
\hline vTo26124 & E & TCAAGGCCAGTGAATGAAATGCAAAGA & CAAAAACAAAATGCCCAAAAACCGA \\
\hline 5126 & E & GCAAACTGCGATCCGTGGAAATTGT & TGGATTGGTTCTGGAATTGGAGGTATG \\
\hline 5128 & E & TTACCCACTGGGAAGGCCAAAAA & TGAAGCATTCAAAGTGGCTGGAAAAA \\
\hline 1 & E & ATCGCACAAACAACAAAATGGGGGT & GAGTTGGCTGATACTITTCGCAGAGTG \\
\hline & & AAAGAGAACGCCGACGCACAGG & GCGCGACTTGAAGAAGCTGATGTTG \\
\hline & ${ }_{E}^{E}$ & $\mid \begin{array}{l}\text { GGGCGAGTTCAGTTAATGCCAAG } \\
\text { GGCCCTGGGCAAAGACTAGA }\end{array}$ & $\begin{array}{l}\text { TCGTATATTCGCACAGGGCAAAGTGA } \\
\text { GGAGTGCTIGGTTGCGGA }\end{array}$ \\
\hline $\begin{array}{l}\text { VIT1222136 } \\
\text { vTO26137 }\end{array}$ & $E_{E}^{2}$ & $\mid \begin{array}{l}\text { GGCCTIGGGCAAAAGACTCAAGA } \\
\text { CAATTTGCATCGGTGCGCATT }\end{array}$ & $\mid \begin{array}{l}\text { GGAAGGTGCATTGGTTTGGCCCTGA } \\
\text { GCAGTGGTGTGGTGGAGTCC }\end{array}$ \\
\hline VTT26138 & E & & $\begin{array}{l}\text { GACAGAGGGGTGGGGTGATGGTCC } \\
\text { CACGCCACCAGGTCATACGGATA }\end{array}$ \\
\hline & E & TTCTAAAAAGAAAAGCAAAACGCAGA & AGGATCGGTTCAGTTTGGTTCGAGG \\
\hline & $\mathrm{E}$ & GTTTTCCACGCTCACTCACCGACAC & TGCACTGCTGCTGCTATTGTTGCTT \\
\hline 60140 & & GTCCGCAAATTGCTTCCTCCTCTTC & СССССtCTCCGACACTCCTCTTTCT \\
\hline VTo26148 & $R$ & CAGGAAAACGCAGAGCAAAAATAAAGGA & CTGGTGTGCCGATGGTTGTGGTAG \\
\hline VTo26149 & E & TGACCCTATGAGCACTGTTTAAATGGAGAA & TGGGAAATATTAATTCGTGATTGGCATAAA \\
\hline 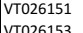 & E & СACGCATACAGTGACATGTACCTGAAAAGAA & СGCTTAGCGCAGTTGGTAACTAATAACTTGA \\
\hline & ${ }_{E}^{E}$ & $\mid \begin{array}{l}\text { ATGGGACACCCAACTCGGGGTC } \\
\text { CTGCCGCCACTCATAGTCTA }\end{array}$ & $\begin{array}{l}\text { GGTGAGGTCAAGAAAGGAGGTICGTA } \\
\text { cagcagcGTIGCAAGGAAAA }\end{array}$ \\
\hline $\begin{array}{l}\text { VIVL2150 } \\
\text { vTo26157 }\end{array}$ & E & $\begin{array}{l}\text { TCGCGCCCAATICACACAGICTAC } \\
\text { САCACAGCATTGTAACATATGGGA }\end{array}$ & $\begin{array}{l}\text { cagcagGGGGTGCAAAAGGACAAA } \\
\text { CAGGTATCTGAGGGGGAGGGG }\end{array}$ \\
\hline & E & AAAATAAAGAGGGGCAATGGGGTGG & cacacacaaacgcaaacacatatacaa CA \\
\hline & $\mathrm{E}$ & TGCAAGGCTTTACACTGCGTGTGT & CGACGTTCCCGTTCTGACTGACTTT \\
\hline & $\mathrm{E}$ & AGCCGAGAGCGAAAAACACAACCA & GCGTTIAGCACAAGGAGACACCCAC \\
\hline 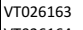 & & TCAACAAGATGCCCACAAATACGAATG & TGTCGAAAATGCAAAAAGGATTTACTGAA \\
\hline VTo26164 & E & GAAGAAAAGCTCCGAGAATTGGGCCT & GGTGGCAACTGAGGTCTCTGCTCAA \\
\hline VTo26165 & E & СCCATGCAGCCGATCCACAGA & сcсаagacacaagacttcaTCTTCGT \\
\hline $\begin{array}{l}\text { VTo26166 } \\
\text { vTT20265 }\end{array}$ & E & TCTCACCCTTGCAGA & ТССТССТССТАСТСАТАСТСТTССТTCСC \\
\hline $\begin{array}{l}\text { VTT226167 } \\
\text { vTo26168 }\end{array}$ & ${ }_{E}^{E}$ & $\begin{array}{l}\text { CGGCTCAGGTGGGGCCTCCTTGGTT } \\
\text { CGAAGAACTGCACTGGGGTAAA }\end{array}$ & $\mid \begin{array}{l}\text { GTCGACTTTGGGCATACCCTGGCT } \\
\text { AGCTCACCTGGGAGGAACAGA }\end{array}$ \\
\hline VTO26170 & ]$^{2}$ & AAGGAAAGCGGAAAAGGGAAGGGAG & GCACATCAGGTGCAAAAAGGTGGA \\
\hline VTO26171 & E & TGGCAGCACTTCGCTTGGTTACTT & TGTTCTCCTGCTCCTGCTCCTCCAC \\
\hline VTTO26172 & $\mathrm{E}$ & $\operatorname{tgtgtgtg}$ & сCTTGACGATGAGTTTTGGGGTTC \\
\hline VTTo26174 & E & CACACACTCATATCGATTGCAGCCAC & САСТTACCACCCAGCACCCGCTT \\
\hline VTo26176 & E & TCGCAGGACAAGTGAAAGGAGCTGT & TGGAAAAAGGCAGAAAATTCGATTGG \\
\hline VTo26177 & $F$ & CAGCAAAGATTTCCTCTCAATTATTTGGACA & GCCTGGATGATGAACTCAAAGGCAA \\
\hline $\begin{array}{l}\text { VTO26178 } \\
\text { vTT226179 }\end{array}$ & $F_{F}$ & ATGATTGCCTGCGAAACTGGGCTAA & TATTGTGGCCAAATGCTGGCAAAG \\
\hline & $\mathrm{E}$ & $\begin{array}{l}\text { CGGCGAATTGGCAATGAAGTAGTAA } \\
\text { CAAGGCAGGCGATATAAGAGA }\end{array}$ & $\begin{array}{l}\text { GGCTCCTGGGGACCACTAACAACTTT } \\
\text { TITGATGAGACGACGGATGG }\end{array}$ \\
\hline 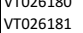 & E & 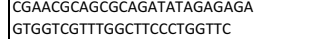 & $\begin{array}{l}\text { TTGGAAGGGGCGACCGACTAACATG } \\
\text { GCCACCAGGATGGTGCACAAA }\end{array}$ \\
\hline & $\mathrm{E}$ & ATTACTTCGGCTTTGAGGCAGGGT & 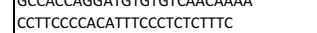 \\
\hline & & Te & ACATAGACCTTCTCGG \\
\hline T026187 & & GCTCCGTCTC & GAATAGGCTACCA \\
\hline
\end{tabular}
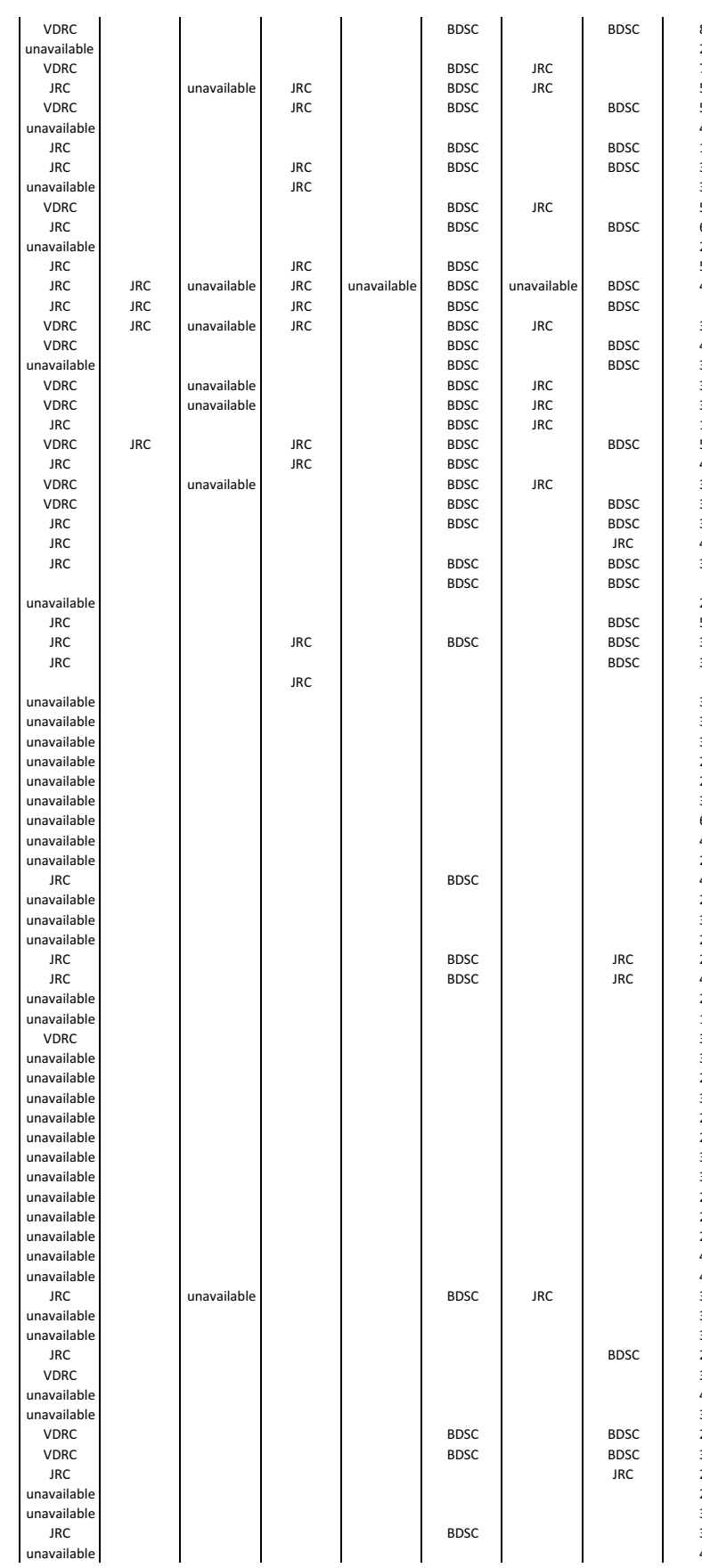


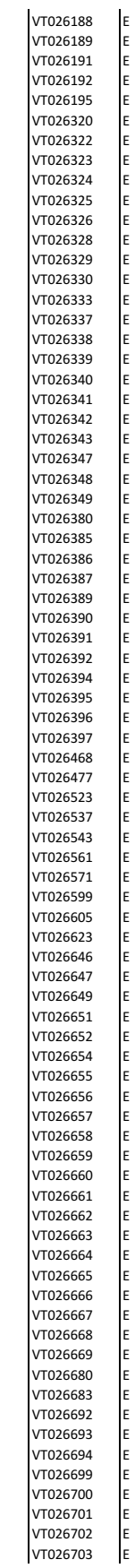

ITCCAACACAAGGGATtGaAatcaAaA GGACAAAAAGGACAACTAGCCGAAAACAA GTCGGACAGCACCTCGTACGCCTC ATCCTACAATTTCGGCACTCAGCA СCTGTTTGTATCTTGGACCGGCG

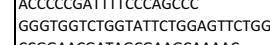
CCGGAACGATAGCGAAGCAAAAC GCCCATTGTCGTTGTGITTCAAGG TCGGACCACATTAAAATCACATCAAATGAAC GCTGAAAGGGGATGGGATGGGATAG CAACTGCAAAGGTTGCCCCAAAAAG
TAAAACACGATGATAGTACTCGGATAATCA GGAGCACAGAGTICCTAGTGGCC TGATGCATTTGGTTGCGAAAATCAG CGTGTTGGCATTCTTTTCAGTCTCT GGGGGGGAGAATAAACAGGACCAAC AATGTAaGCAAAACAGACACAAACGGAA gGaACCACCGAaCCCACAGCAGATA ACCGGTACCCGAACCGGTACCC ATGGGTTCCGTTTCCTTCGTTGG GAAATCCCCAAGGAGCCCGC
GGCTCCAGCACTTCATGGCTACA TGAAAATGTGCAAGAGAAACTTACAGGAA AACGCGCGACACAGTGGGGAATAG
TIGTCTAGCAATTGTCTACAATAGTCCTGCT GCTAAGGGTTGGAGGCAGGAGGA AGAGCGAAACCGAAACTGAAACCGA AAGAGTTGCGAGTTGCCGAGAAAG CGTCGAATGCGTAGCAAATTCAGCA
CGGCTCCTTAAACTGTCACTCGC GGCGCCATCTAGGGCCTTATACTTC CAGGAAGTAAGCGACCTACAAGCG GCTCGATAAGTTTTGTTGAAAATTGCTCA GGGCAGAGCCTTAGATGCGACTCC

TСACACACTCGTTCATAAGTGGCTTC TTCATAAGCTCATTACACACATTCCTC
CTACGACGGTATGGGITGGCTAGG GCCGCATTGCTCAGGCTATCAAACTC GCGGATTACAAGGGGTGTCCGTIT GGCTGAATGATTATGAATAAGTGTATGA TATAGCAGCCAGTICATGAGGGCCA
GGGATACTGCAAGGATTGGCCGAT GAGGACTTGACCATGCAAACGGATGT TTTTGATAAGTTITTGGGGGCACT CAGGGGCAACTITGTTGTGCATTT GTAATGCAATTCAGGCCAACTAACGA CAAAAATATGGGCCAAGGCTGCG GaAGagGcatgattGgataAcaAath CGCATTGCATACTTCTGGACGTGGT GGGGGAGAGGTAACCCACAC TGATTGGTGTTGTCGTTTTGGTGGT CCCCATCCAACCTTCGGTGAAATTA AGCTCATTAACCAGGATGGGGGAG GCAGATGAAAGGGCAACGGGAAC TCTTGACTGAATATTAGACATTCCAAGTGCGT TGGAGAAATCCACACCCTCCTCCA CCAAGTTGATTGACTACGGTAGGGGG GAGCTTGGAGAACTCAGGGATTGCG CTCCAAGCGCAAGTCCAAGCAGA GACAGGGTGGCTGCTGCTTGG CTGCTGGGGACTTCGGAGAATACG GTGGGGCAATTAAGCGGAAAGGG GGGGGATAAGCAAATGCCGAACA
GGGCCACATGTACTCCAATTCC
|TGGTGTCCTCTATCTGTCGCTGTCC GCTTTGGCATAGAAAAAGTTGTACGGAA CGCAGTCAAACGTTTICGAGATACAAC GGCTCAAAACCAGCCACTTGGTACATC GCCCCGTGGATTTCATCCCTCT

TCGGTGGGTITCTCCACTACATGA

CCCATTGTGACTCGTCGTTTTTGG

AGGTCACTCTTCATTCGCCGCCC

(CCATAAAC

GGTGCCTGATGCTGACACCGA

CTACTITCCCGCCAAACCACACATC

CAACATTAAAAGCAGCCAGGTGGGG GAAGCGGATTCCCGCGGAGC

CAAGAGCCCAGGACAAGCTCG

AatAAGGCCGCCATGAAAGTGCAGG

ACAGCAAAAATGAATGGGAGTGCGA GGaGGaGGITICGGAATACAAagata TGCAGTGTGTCCAACGGATTCTCT CAATAAATAAAGCCTATTGCCACTCGATCA GAAGTGCATTGAGGACAGGCAGCA AAGTGGTGTCCGCAATTGAATTTG GAGGTGGTTGGGTGGATTTTGGG GAGGCATTGAATCGGACCATTTGA CCACGGGGGTAATCTATGACGCTTG CCACCTGCACCGCCCACTCC TCAGCATGGGAACTGGTCTGGT GGAACCCAACCCAAATCACACTCACA AATCCGAAAGCCATAGAAATGCCGA CGTGAGCTAGGGCACCGAGTIAAAAG IGGTGTAAACTAACCTTTACACTCGCCT GAGACAGAAACAAATGCAAATCGGT GGCTACGAAATCCTGGGAAAACAA ( CGGCTCACACAGATACTACACACCCA

TSCTTAGCGAGAGCTCGTTGGGTT AATGCAGCTCTCTCCCAAGTCTTTTGT CTGAGATAAGTGGGAAAAGCGTGAAAAGC TGTGATITTGCTTAGTCTTGATTTTTC GTGGAGGGAAAGAGAGCGGTGAAA GITTCGGTACGGTTTGCATTCCCC GGACGAAAAAAAATGCGGGAaAa IAATAAGTCCCGGCAATCCAAGCGG CAGCATATGTTITCCCAGTGCAGGA

gcggcaggaaACAAAGGCAAC TIGACCATGTTAAAAATTGCAAAGAAAGA TATGGGCAGAAATCCGGAGAAAACA GAGCAAAAGCATAACGACTCCCAC GCAGCCAAACGGTTAGCCACTTCAC CATTCCCATAAGATGGGCGTGTTCA CAAAATACGAAATGCGAACGAGGCG AAACCAATCGGCACCAAAAGTGTC
AGCTGGTGAAGGCGGTTATTIGT CAAAAATGAACATAAAAATCCAAAGGAACCCA
TCATGCTCAGAAGAAAACATACAAATCGT

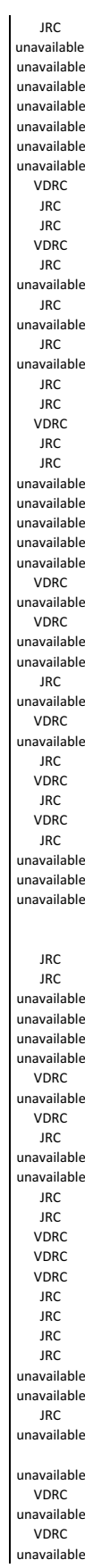

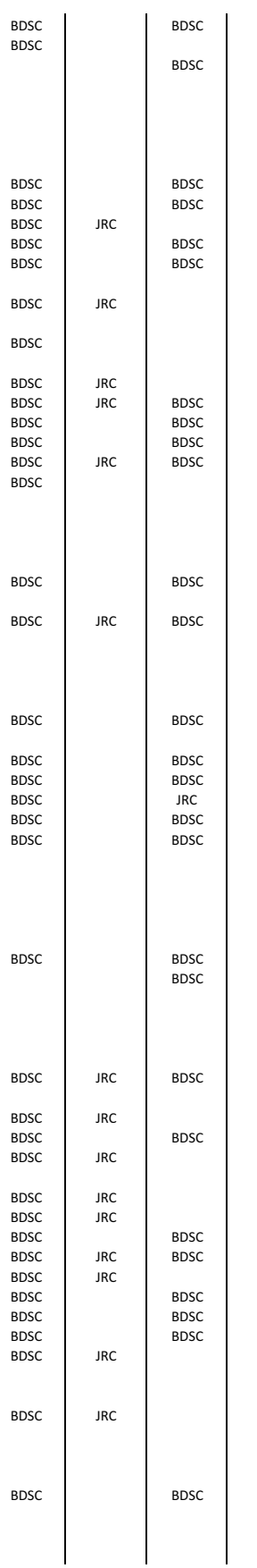




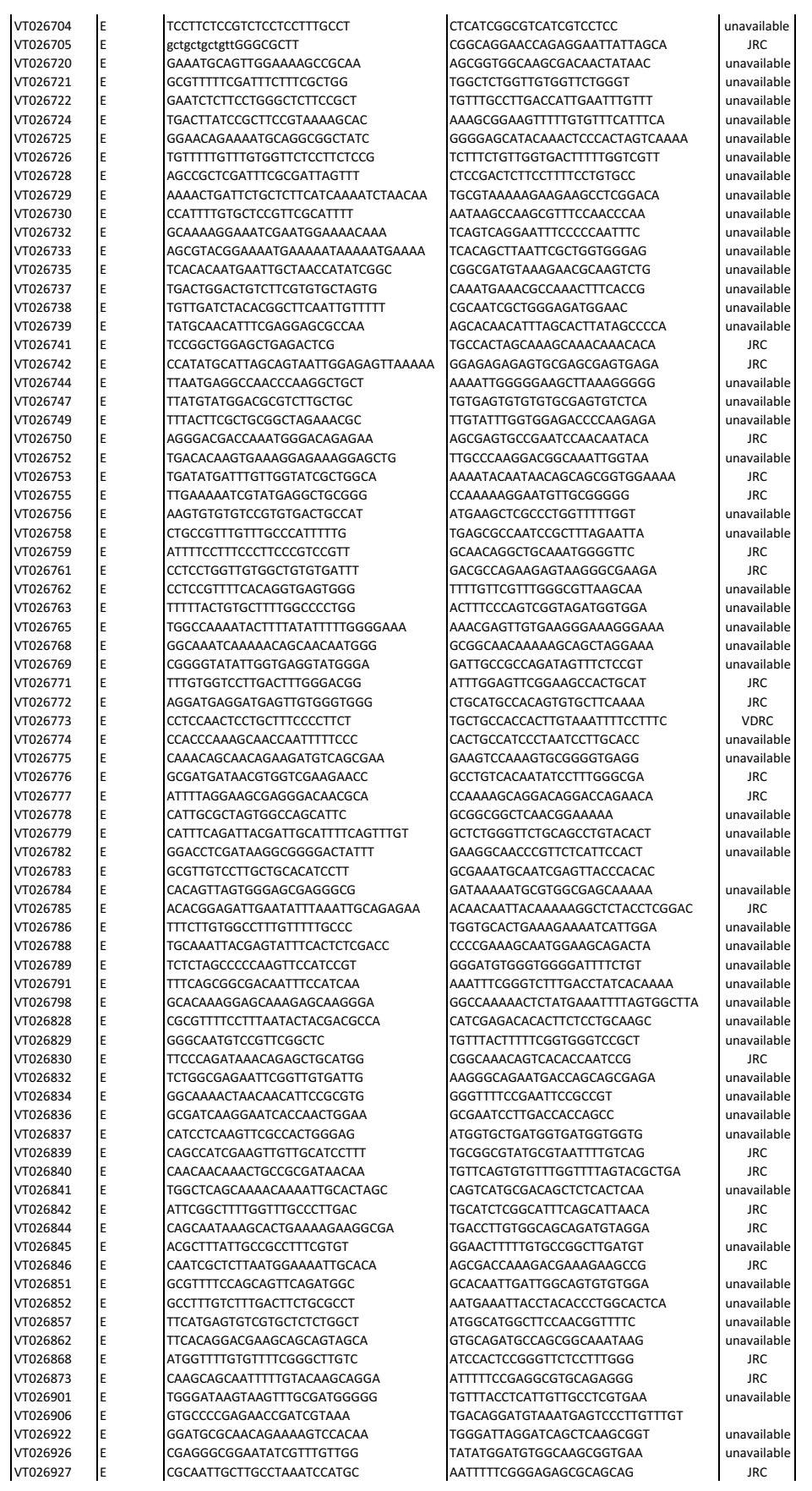
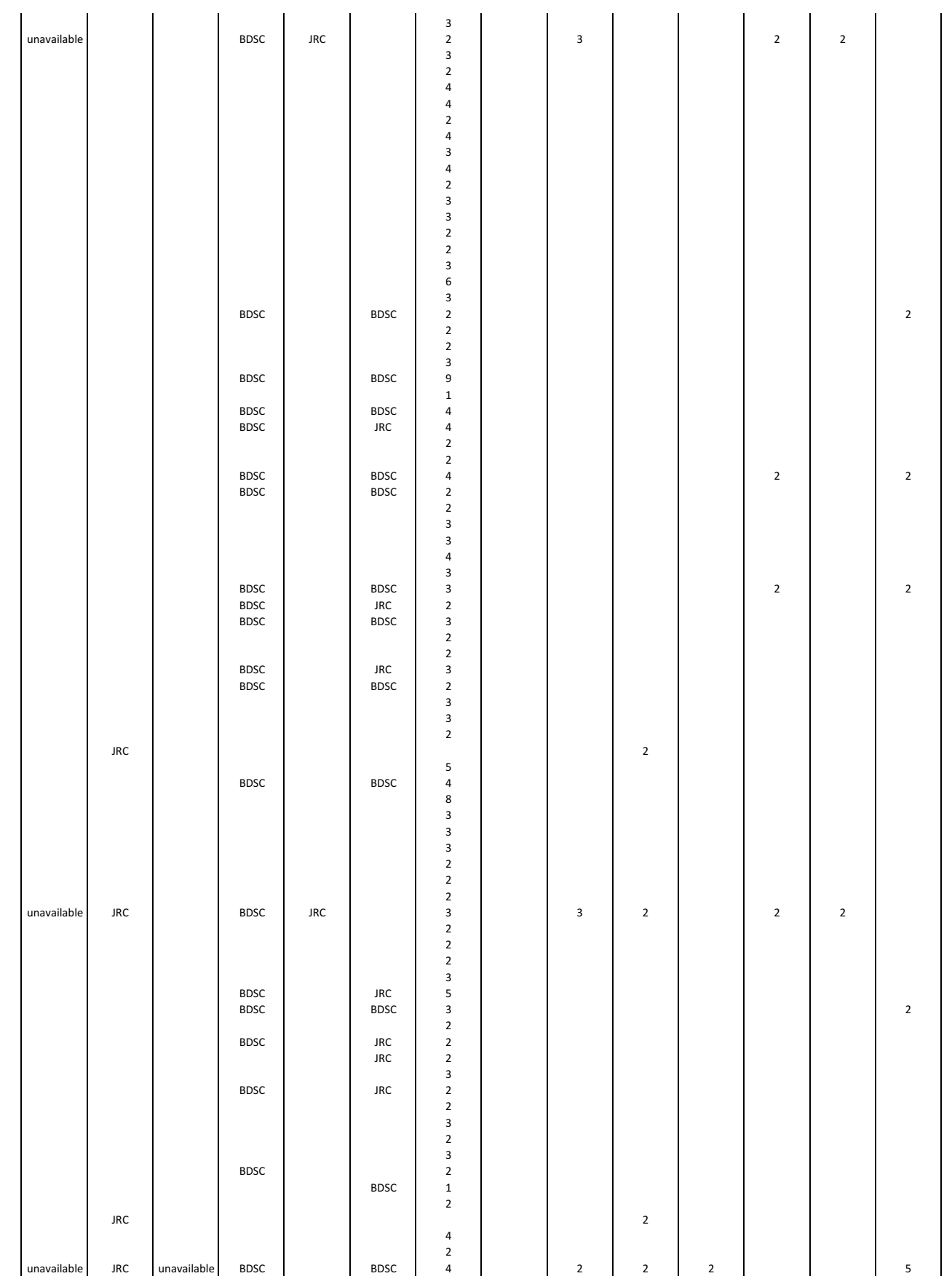


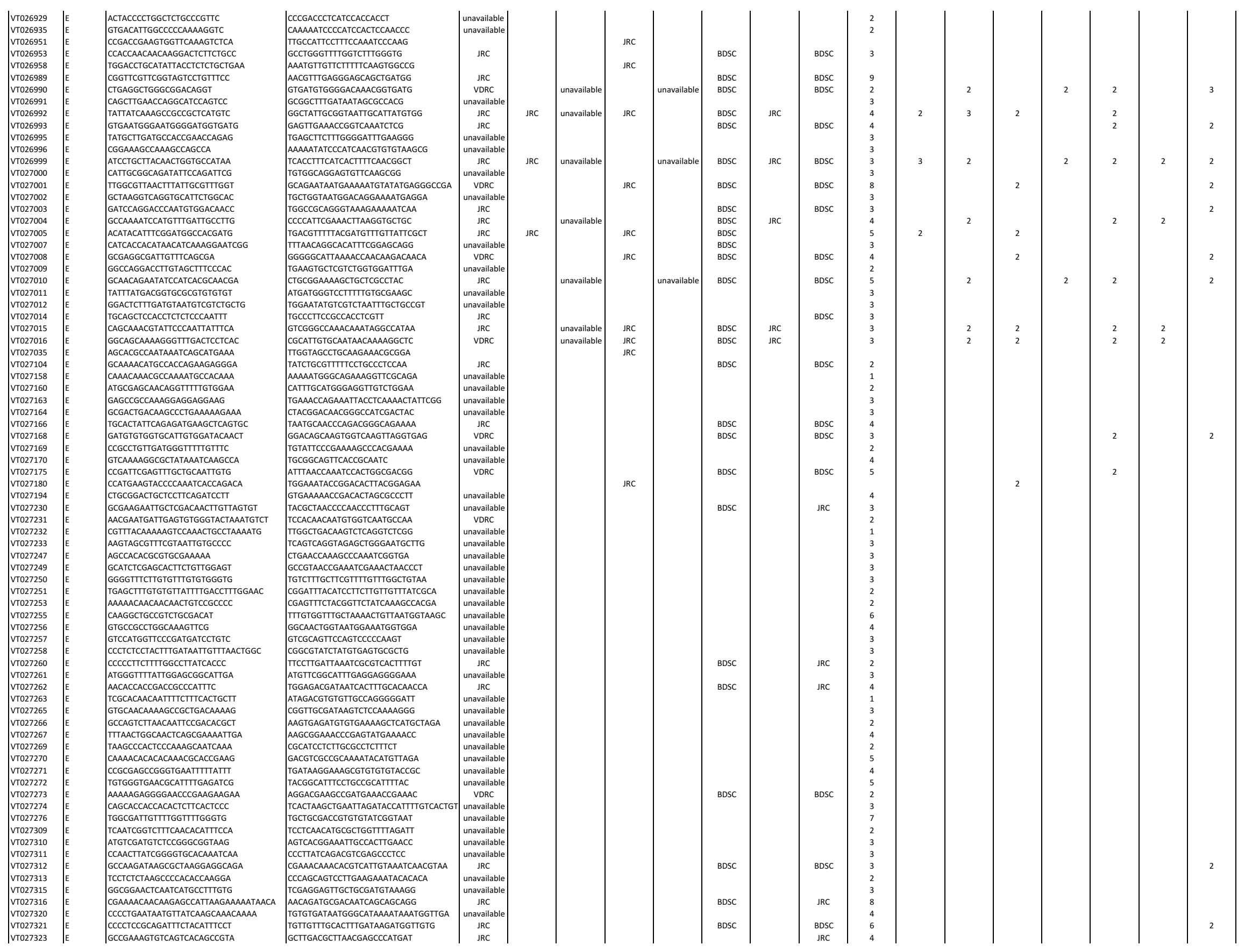




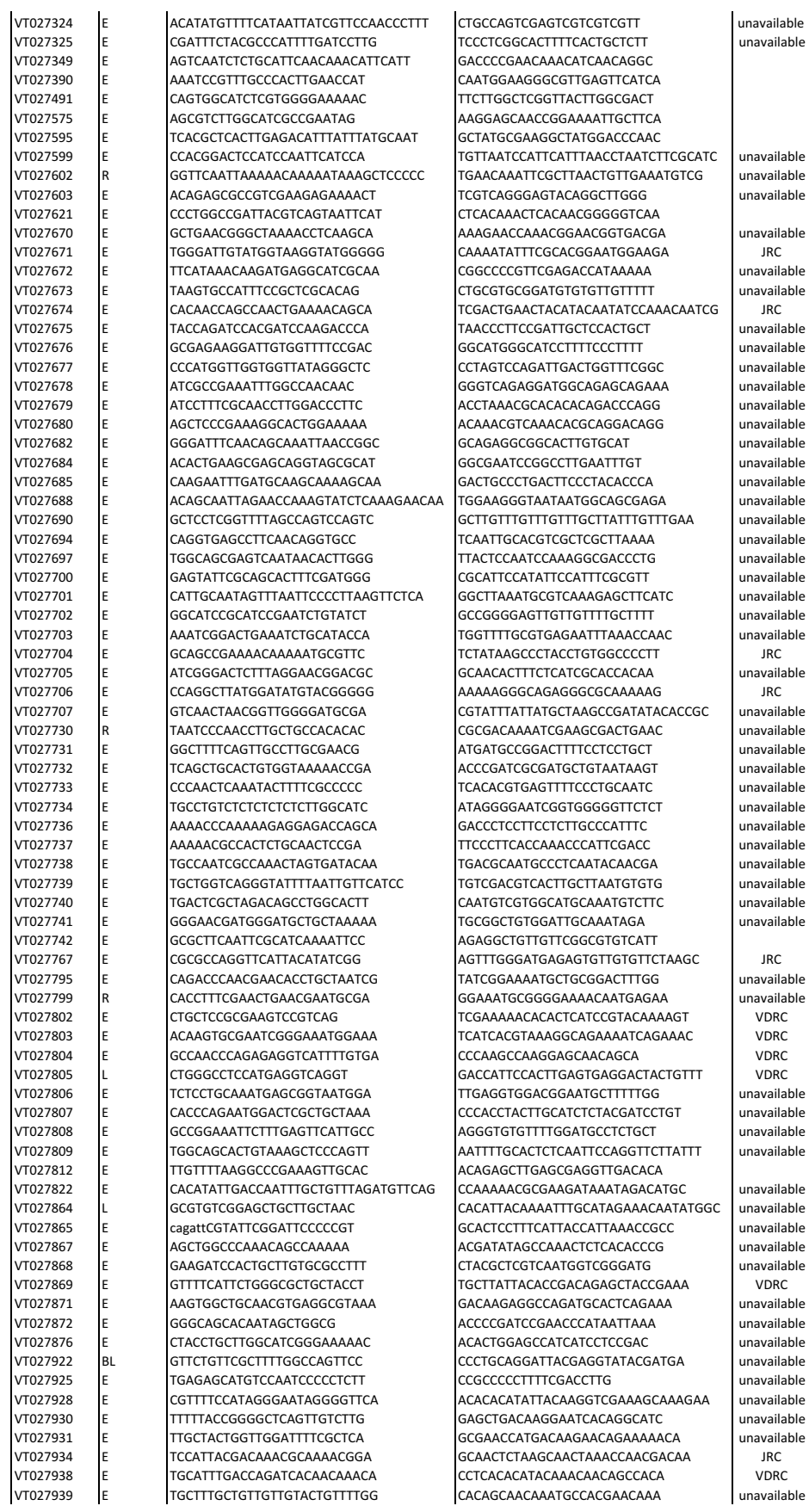
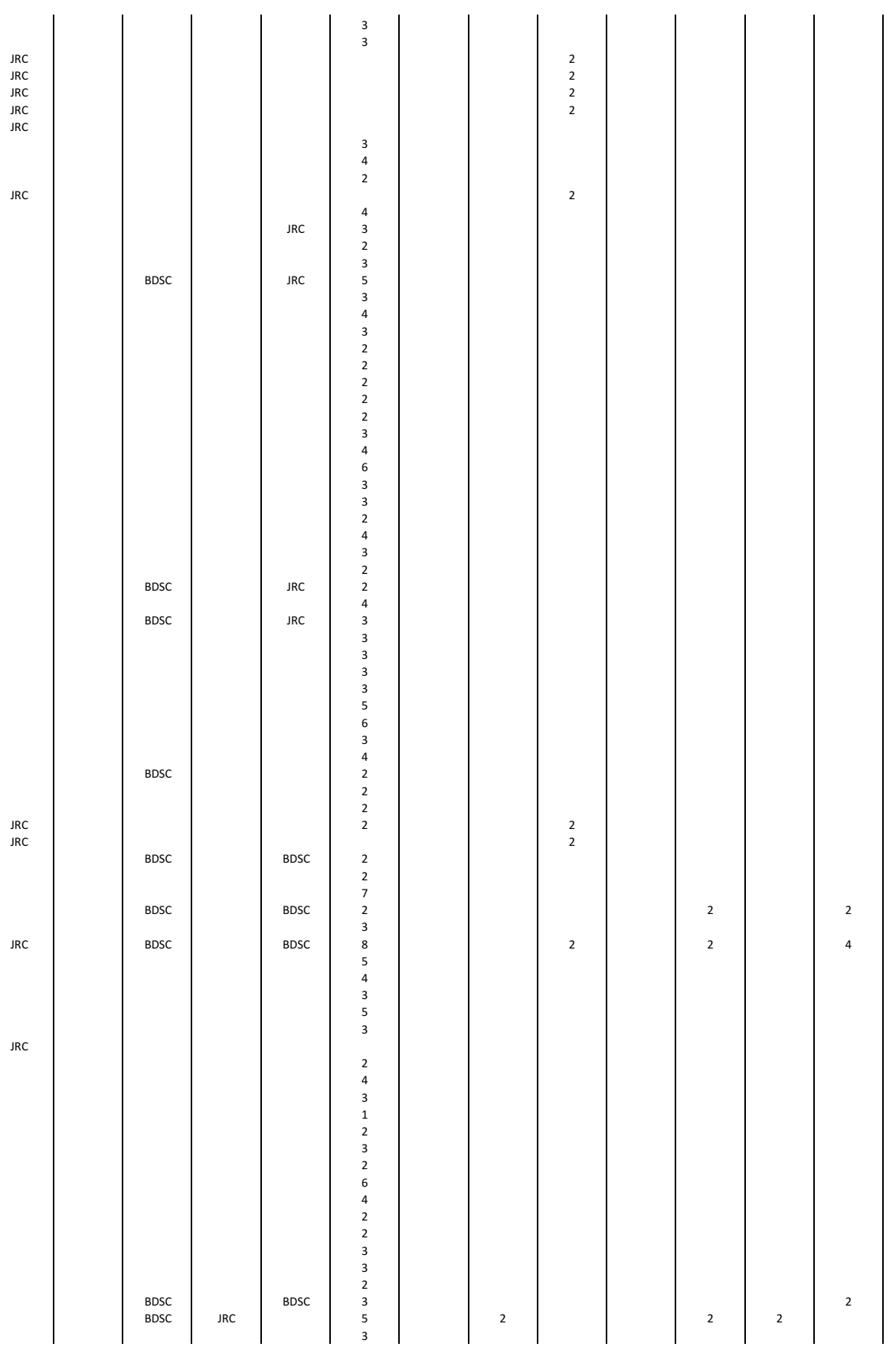


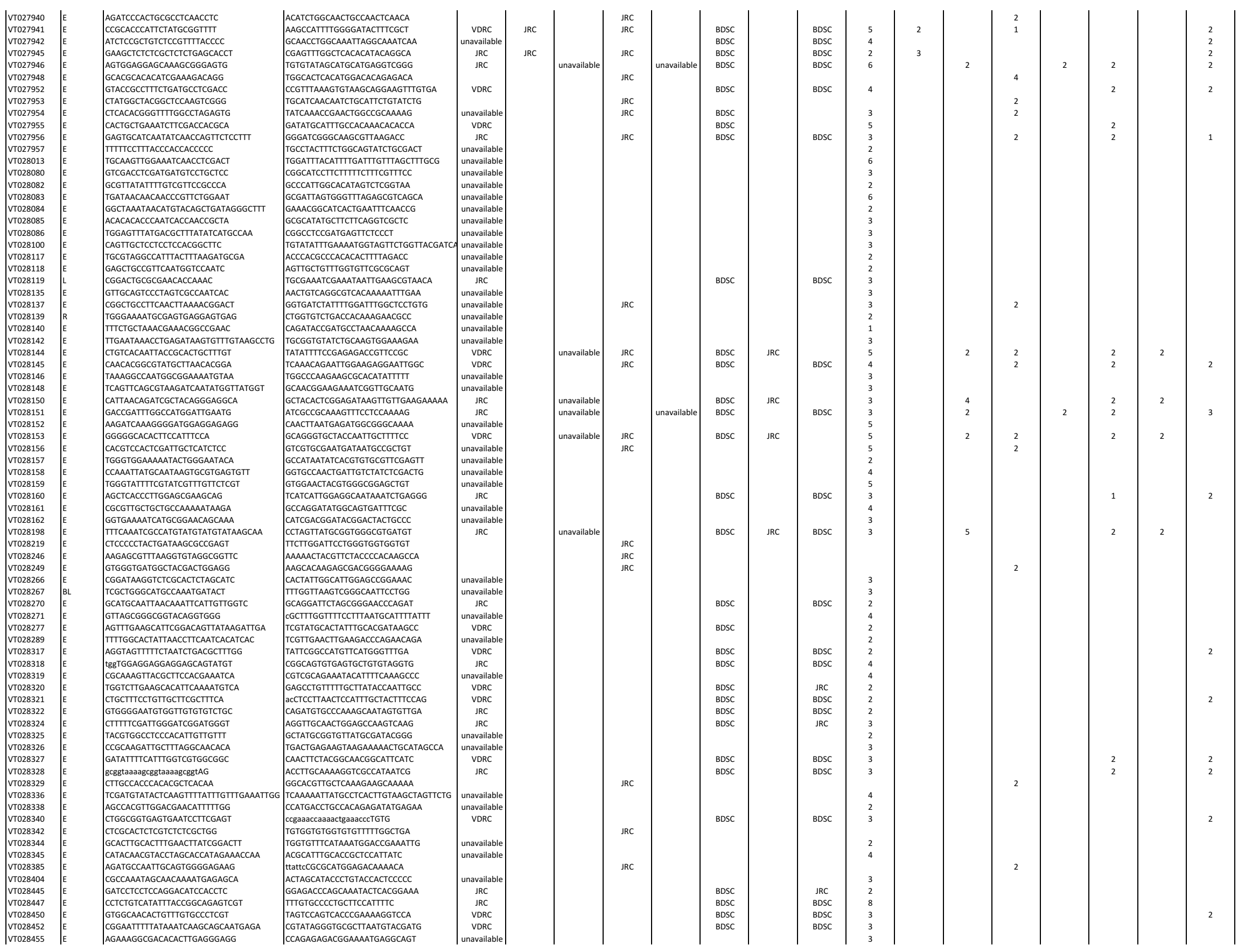




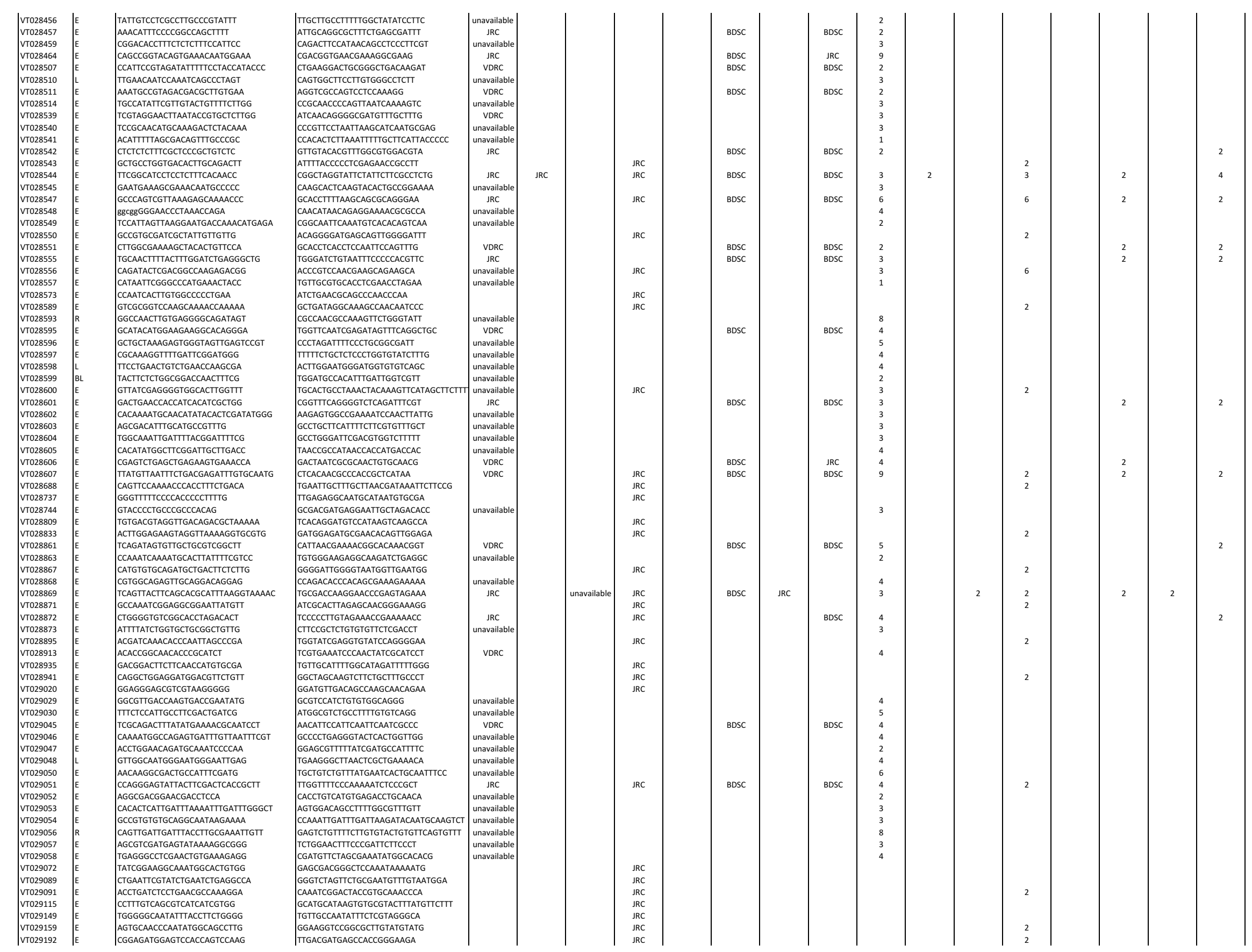




\begin{tabular}{|c|c|c|c|}
\hline & 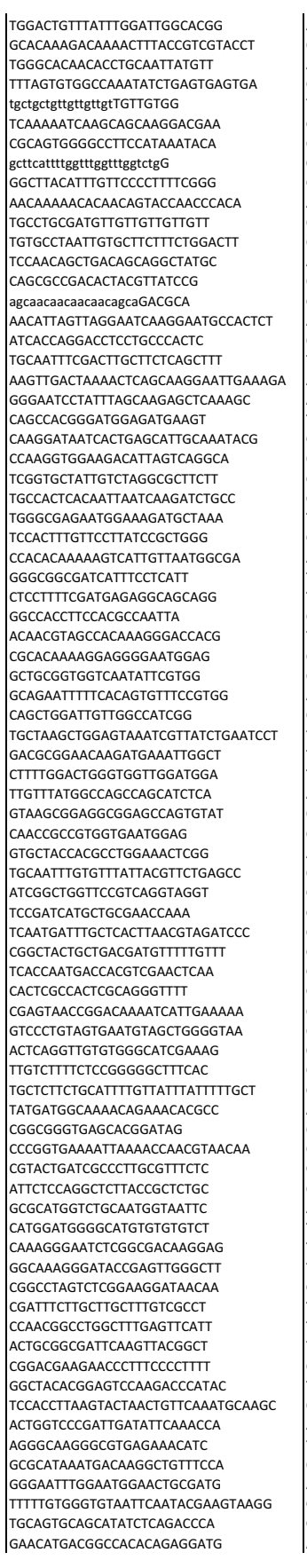 & 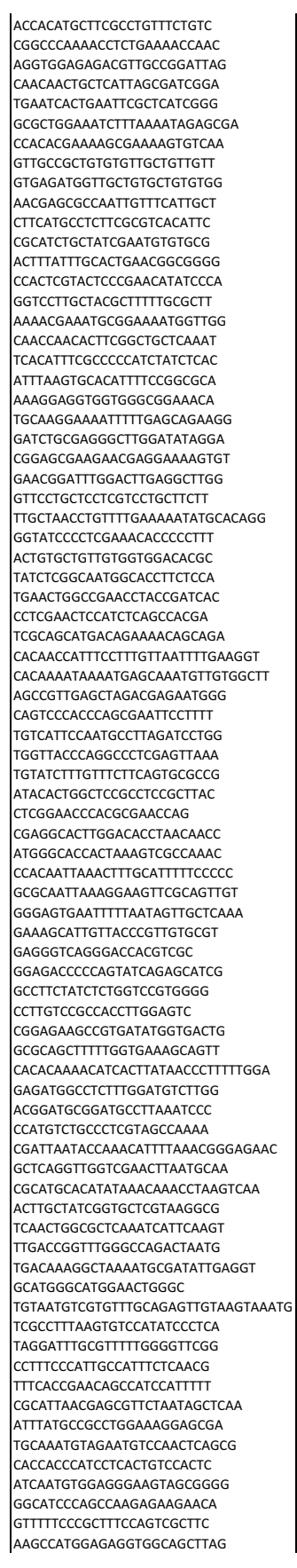 \\
\hline
\end{tabular}
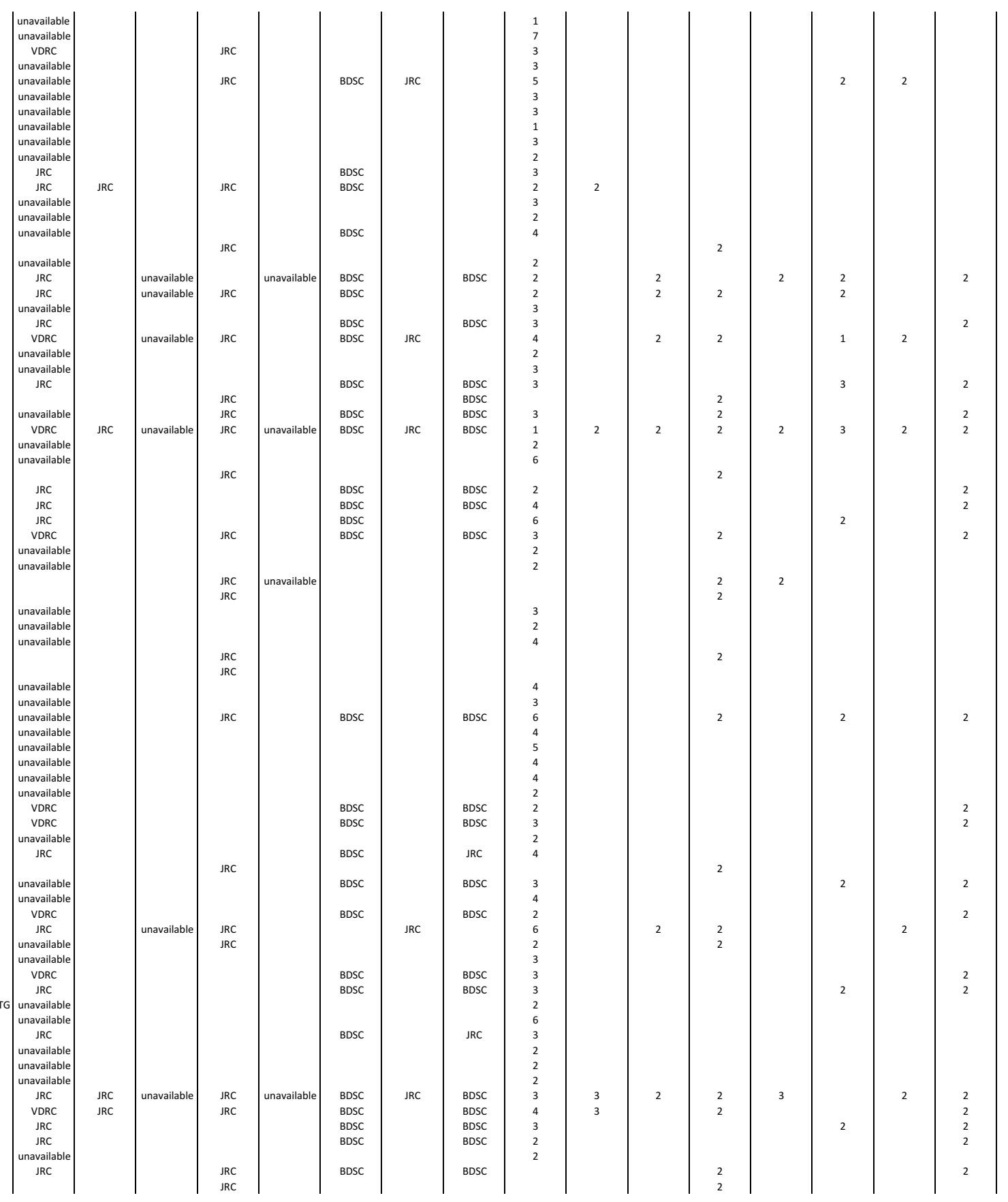


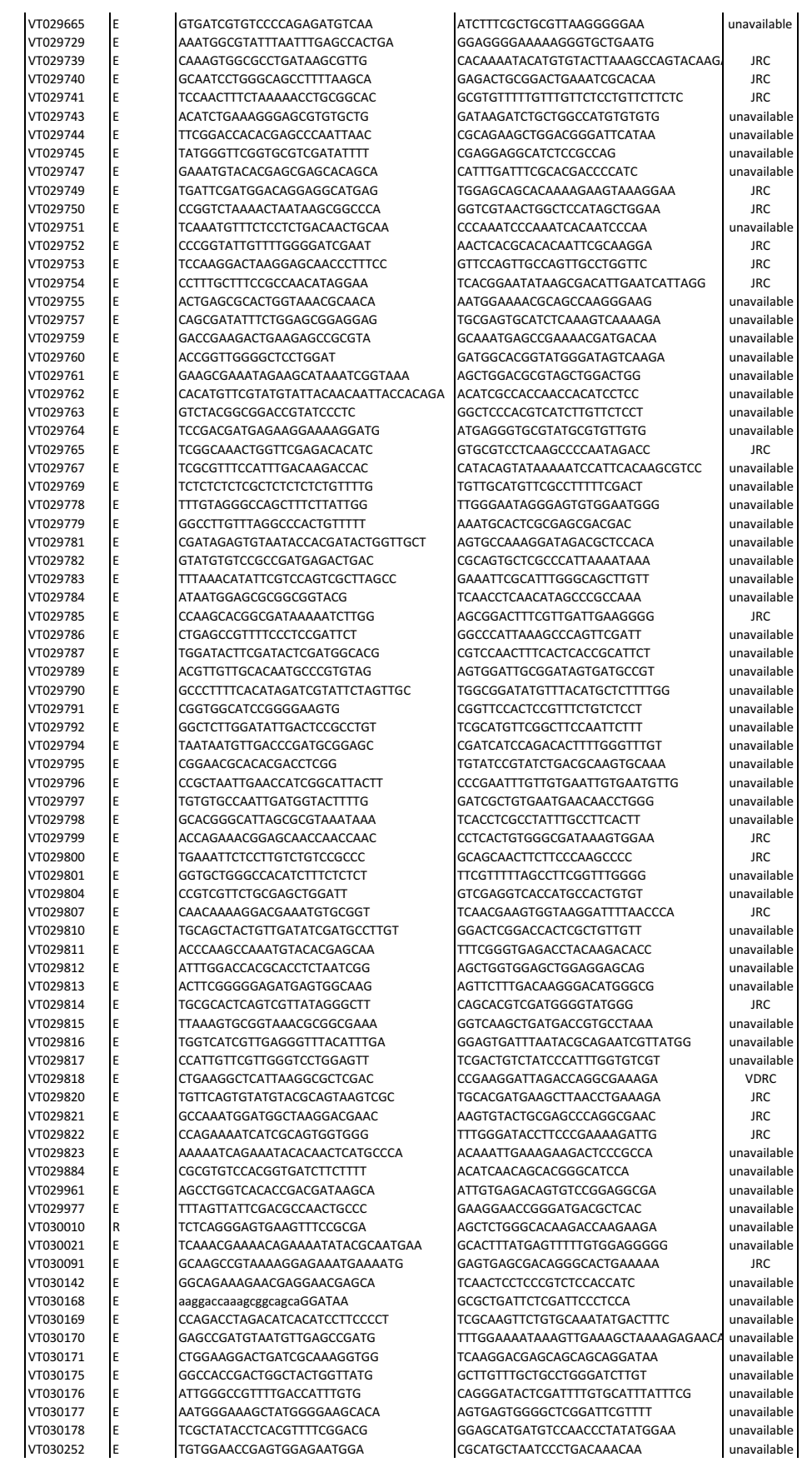

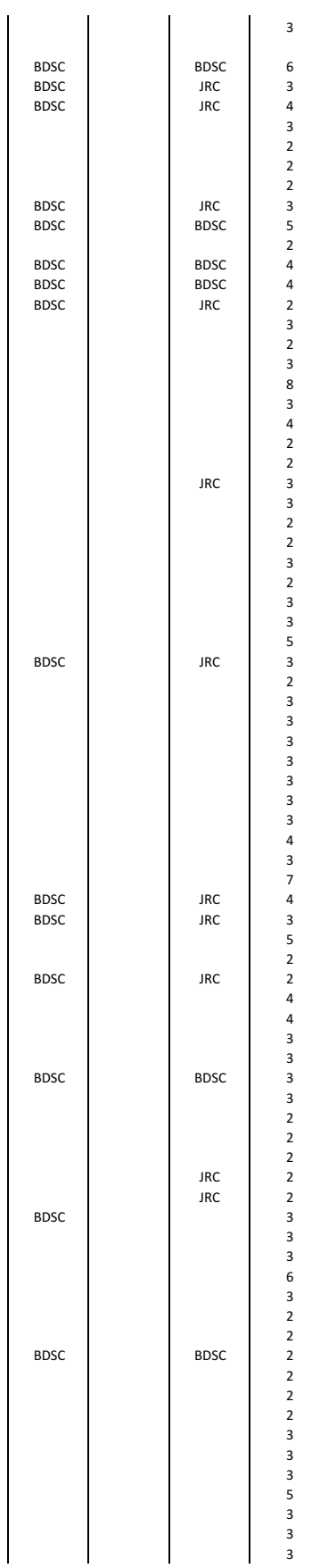




\begin{tabular}{|c|c|c|c|c|}
\hline 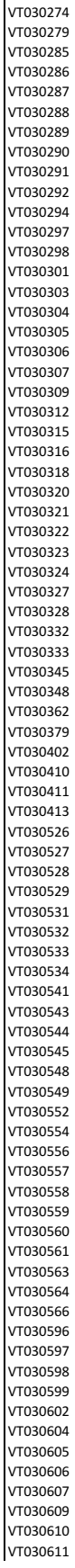 & & 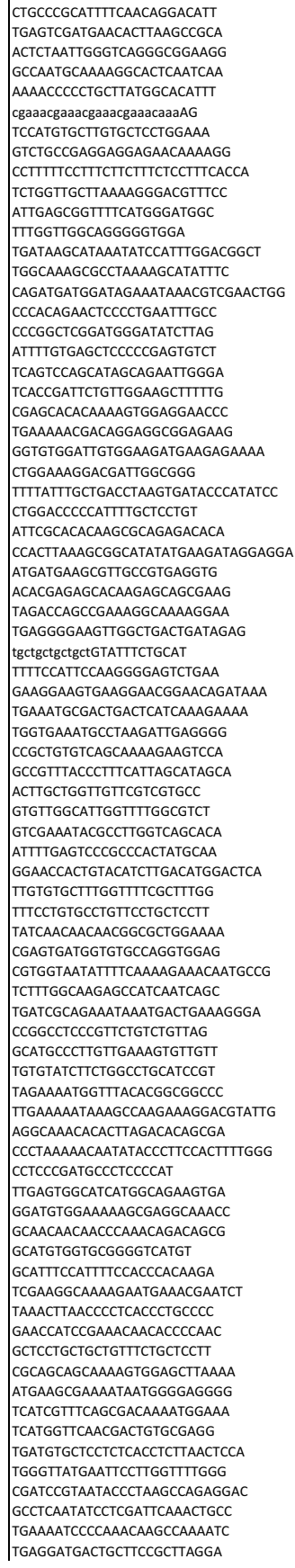 & 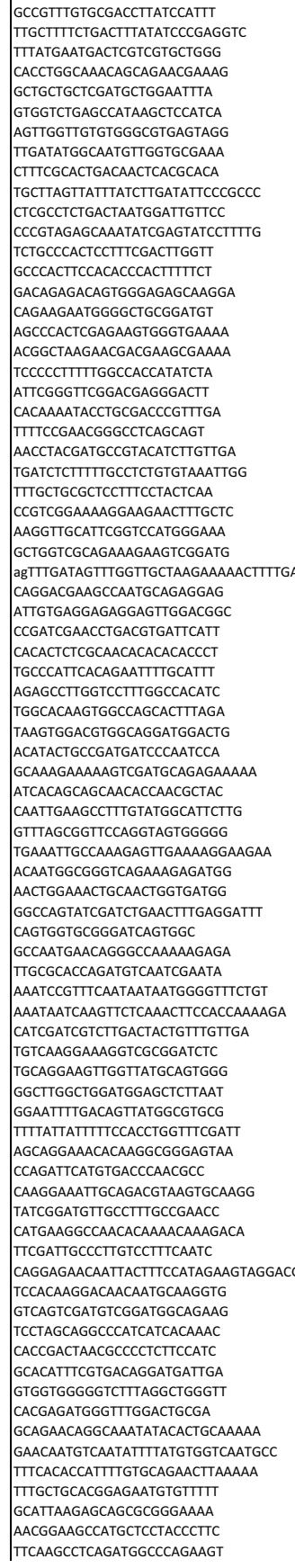 & 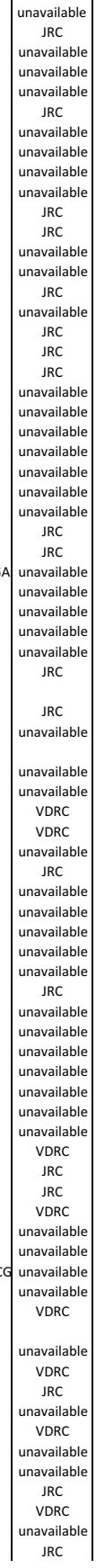 \\
\hline
\end{tabular}
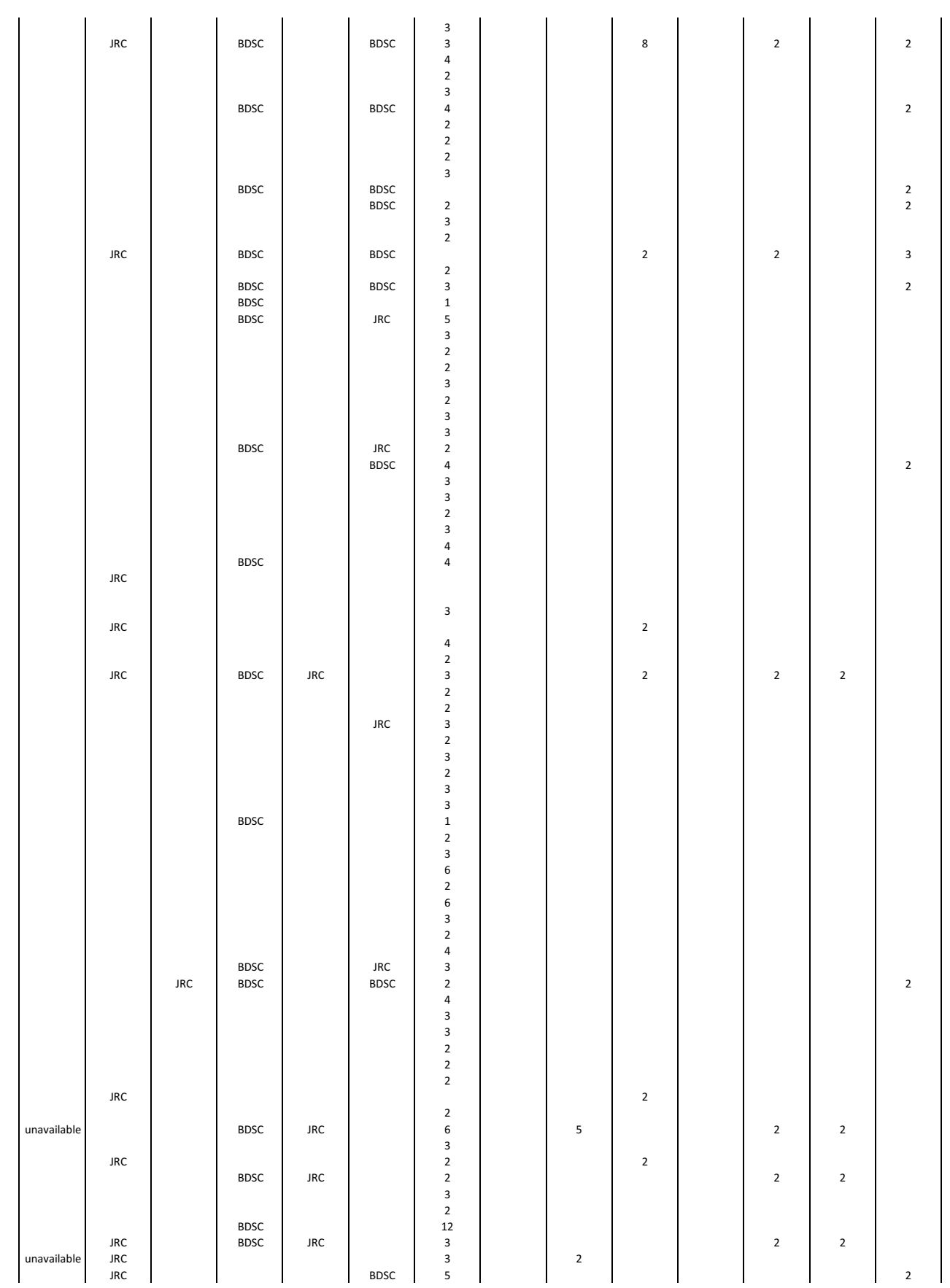


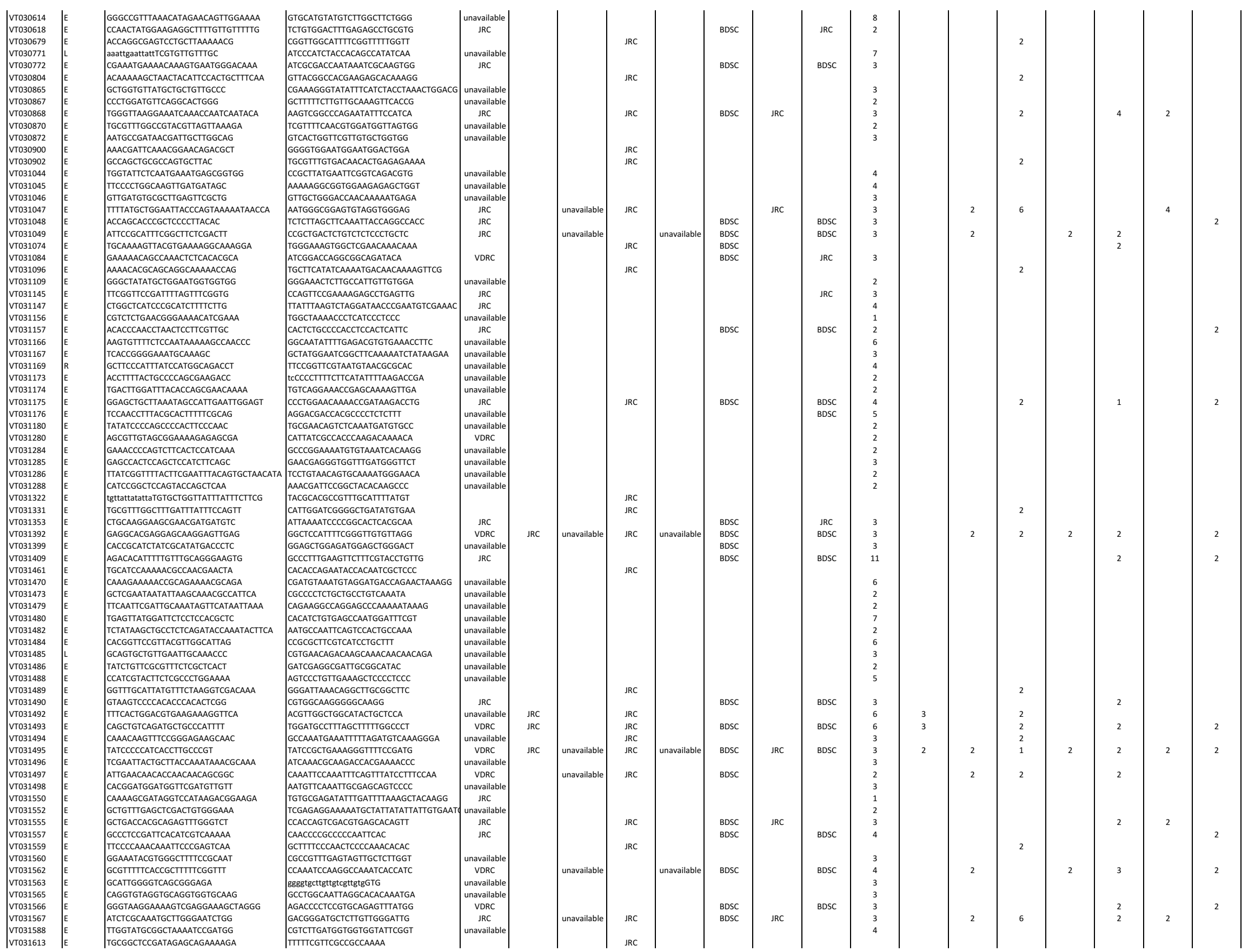




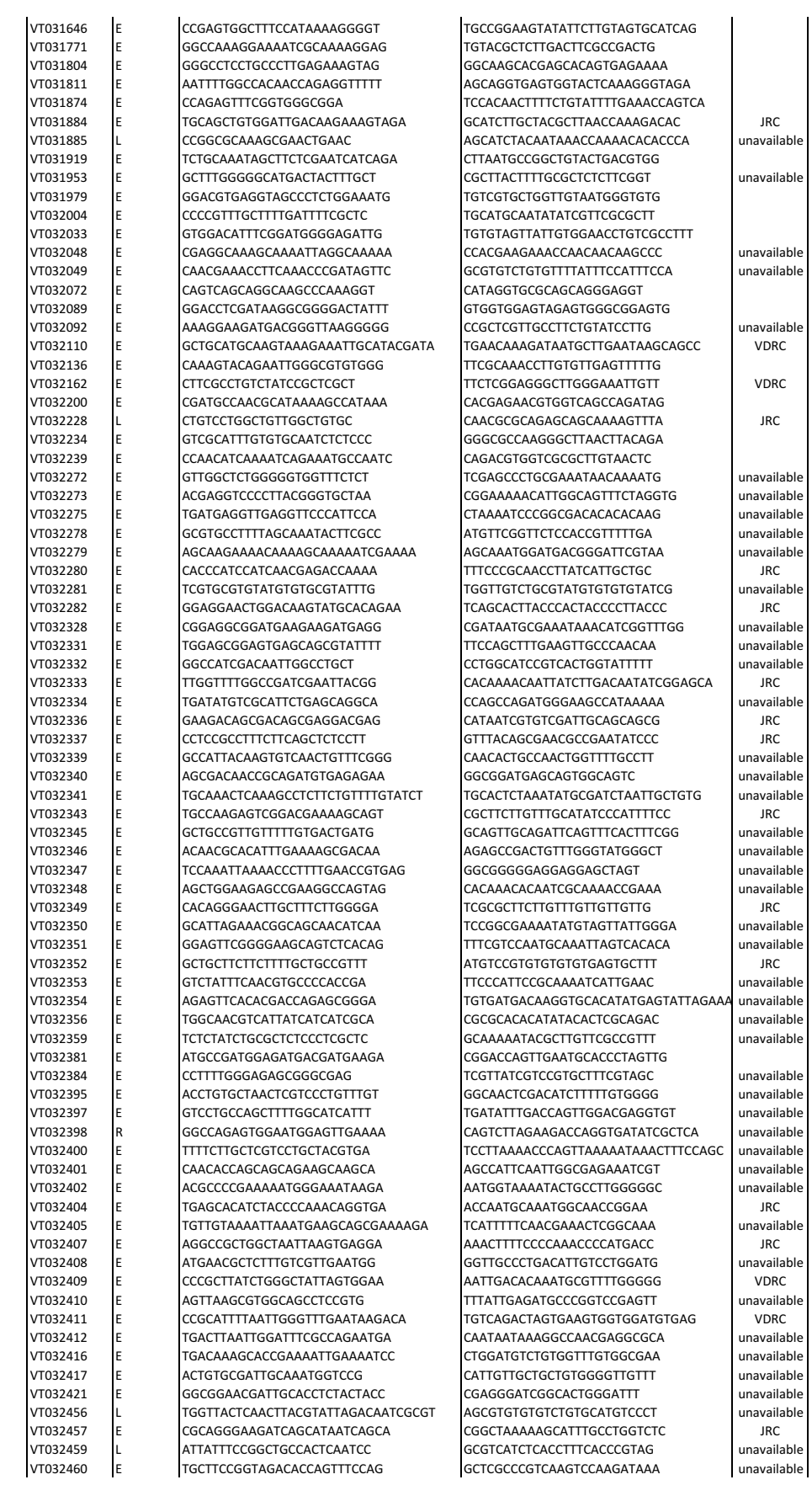

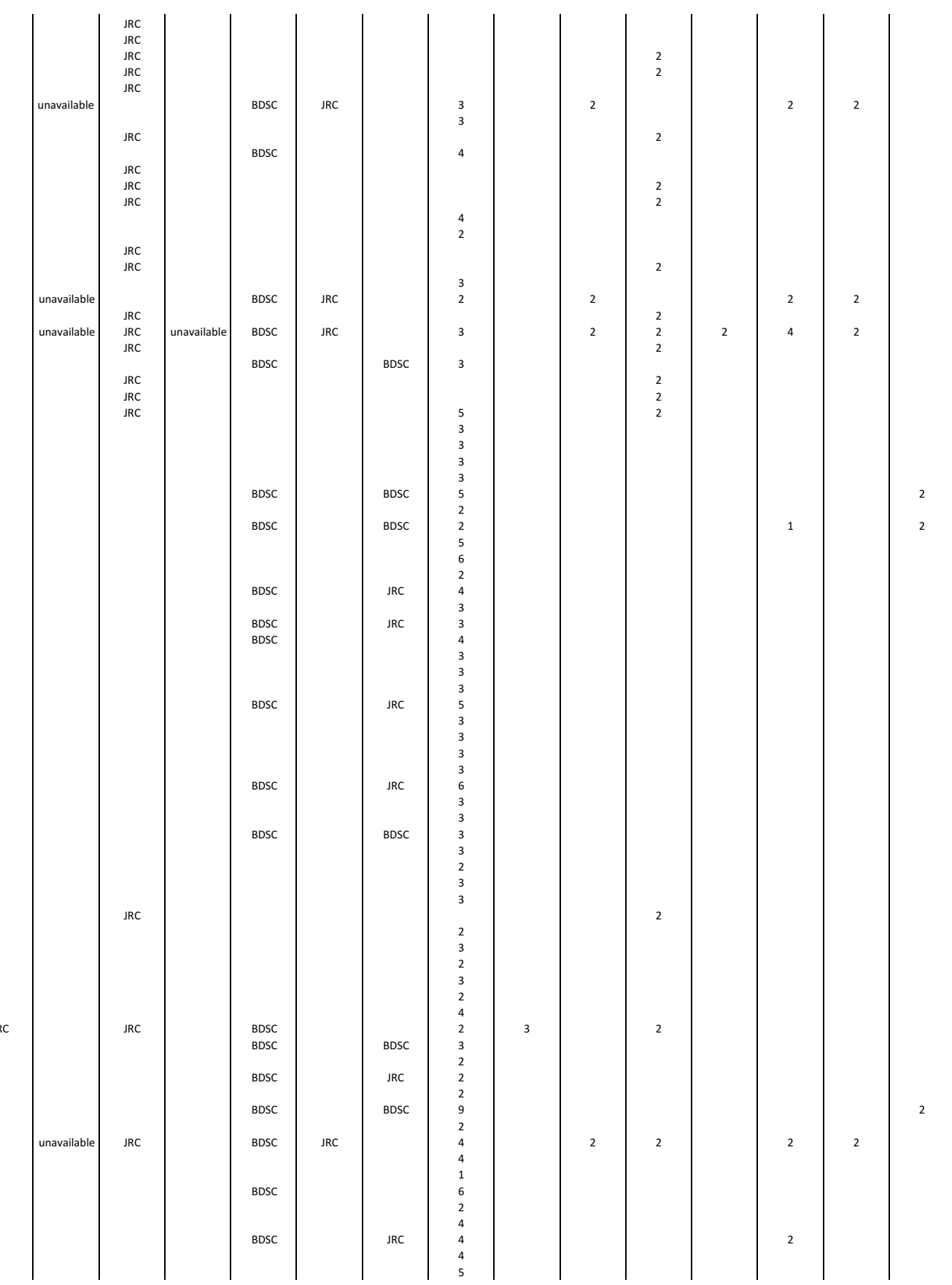




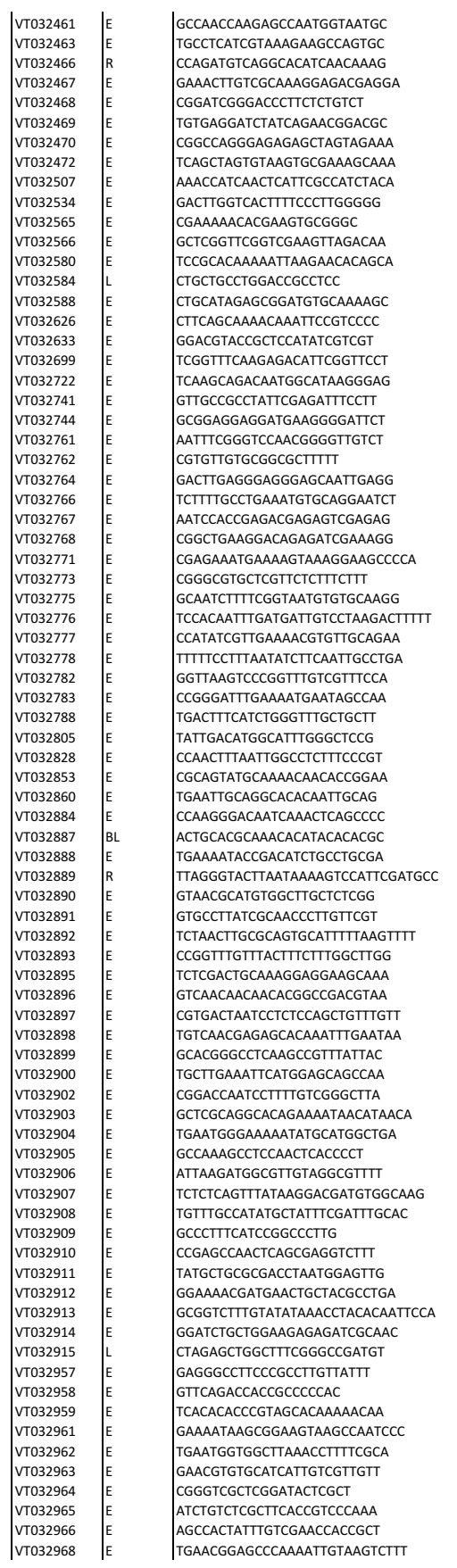

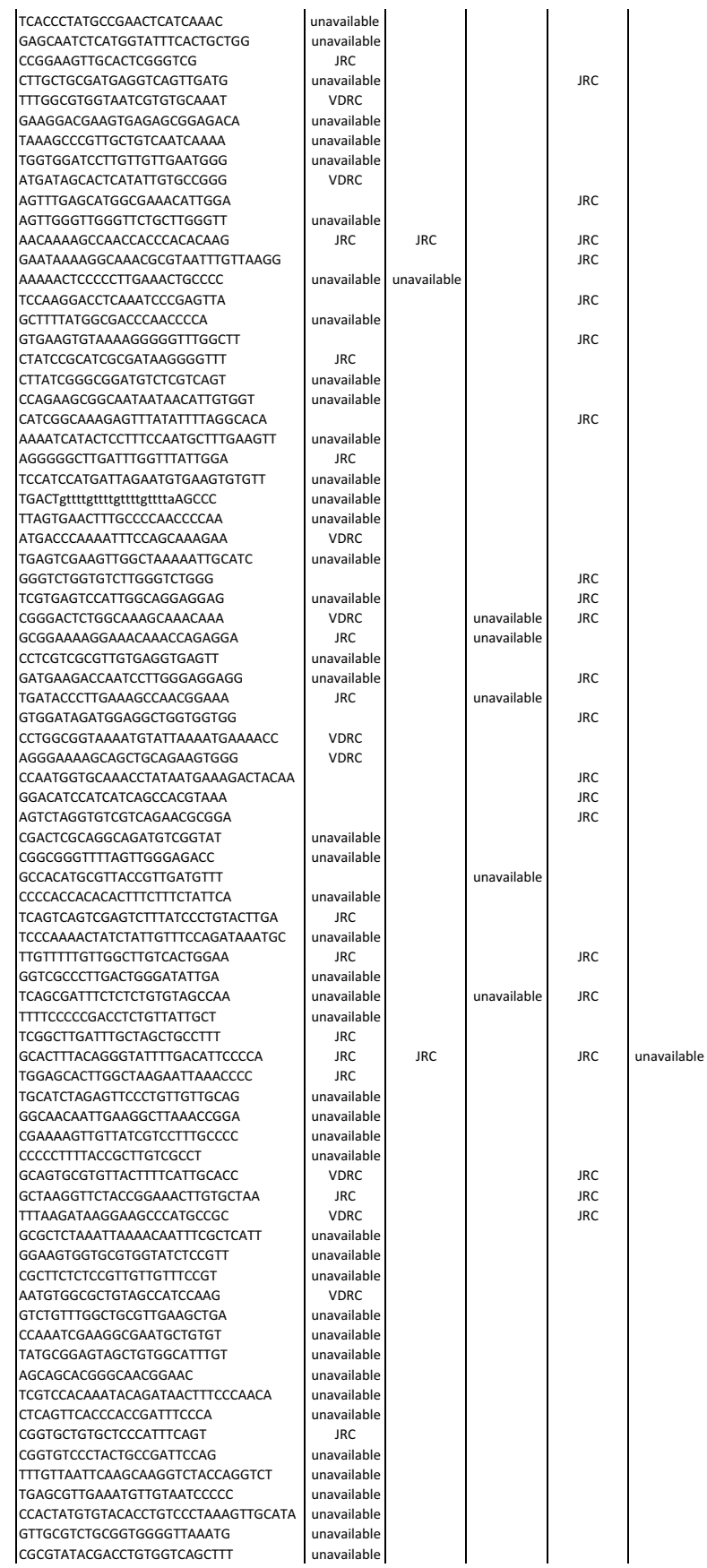
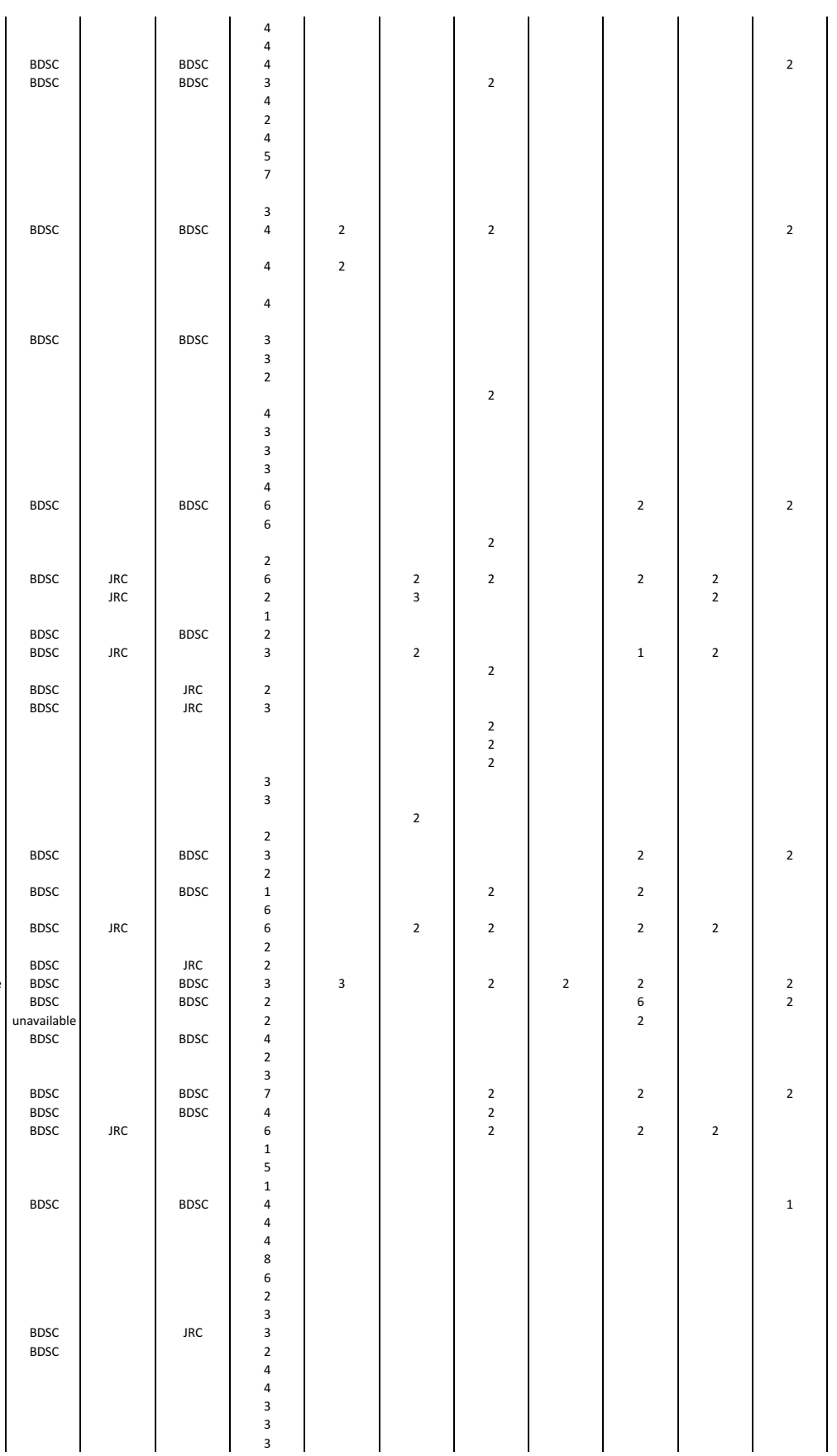


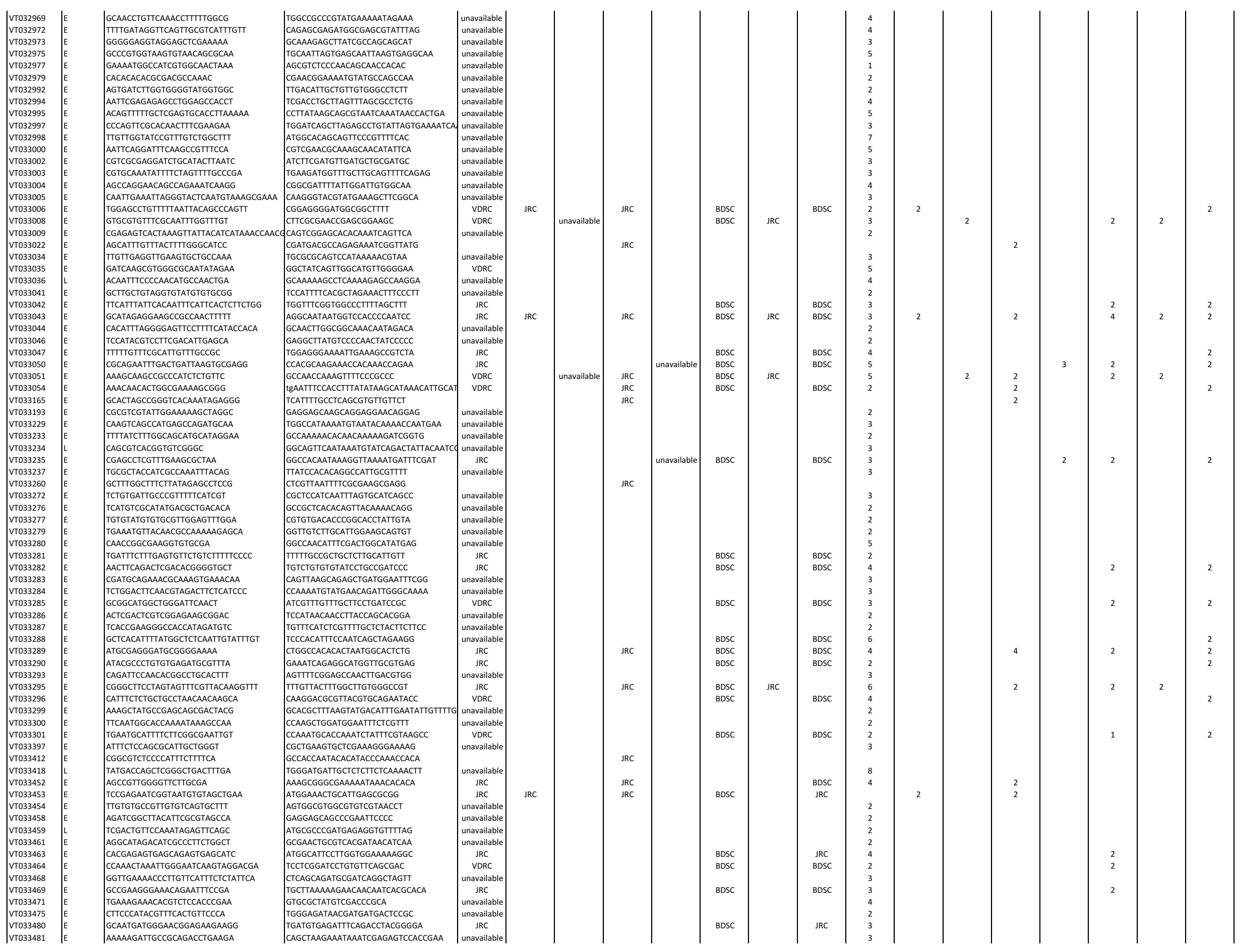




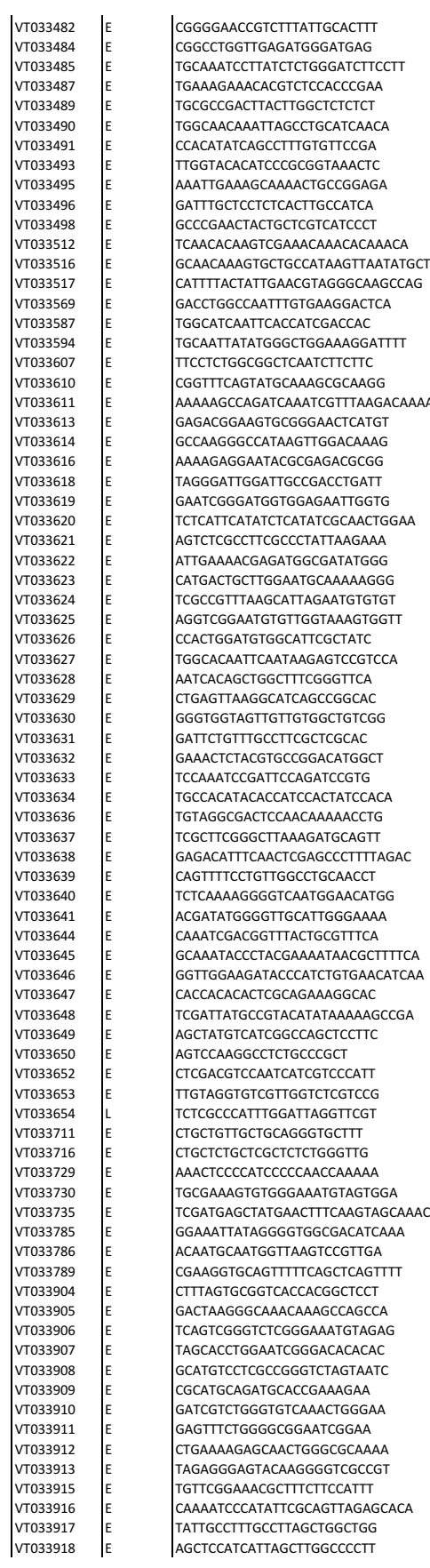

\begin{tabular}{|c|c|}
\hline 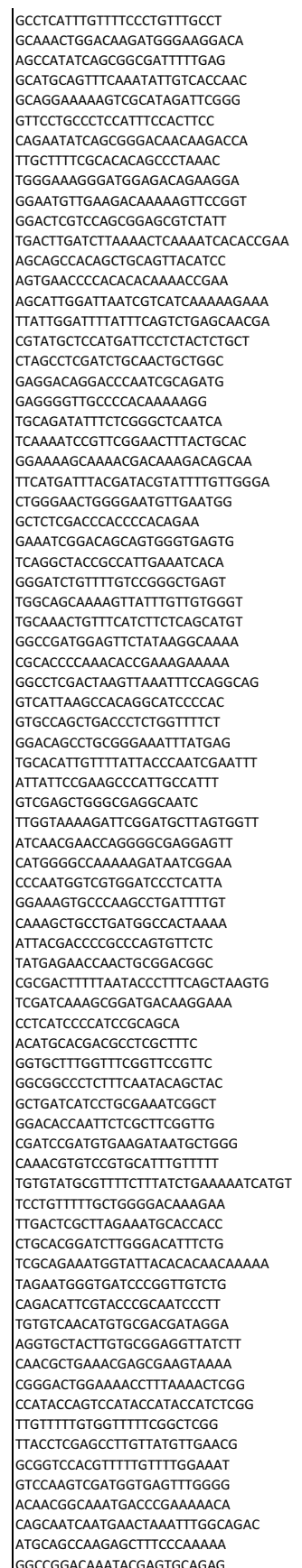 & 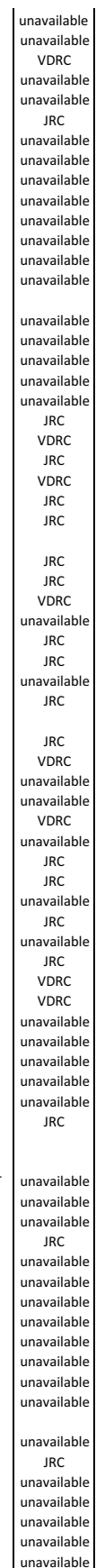 \\
\hline
\end{tabular}
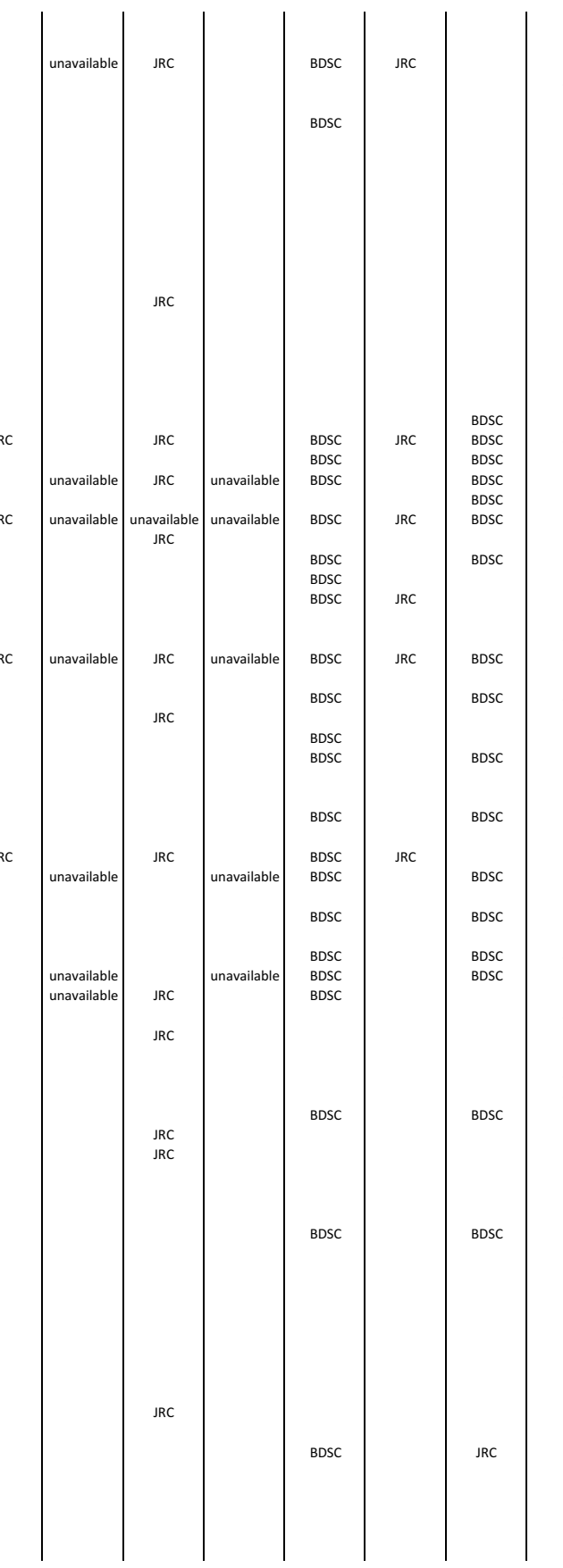


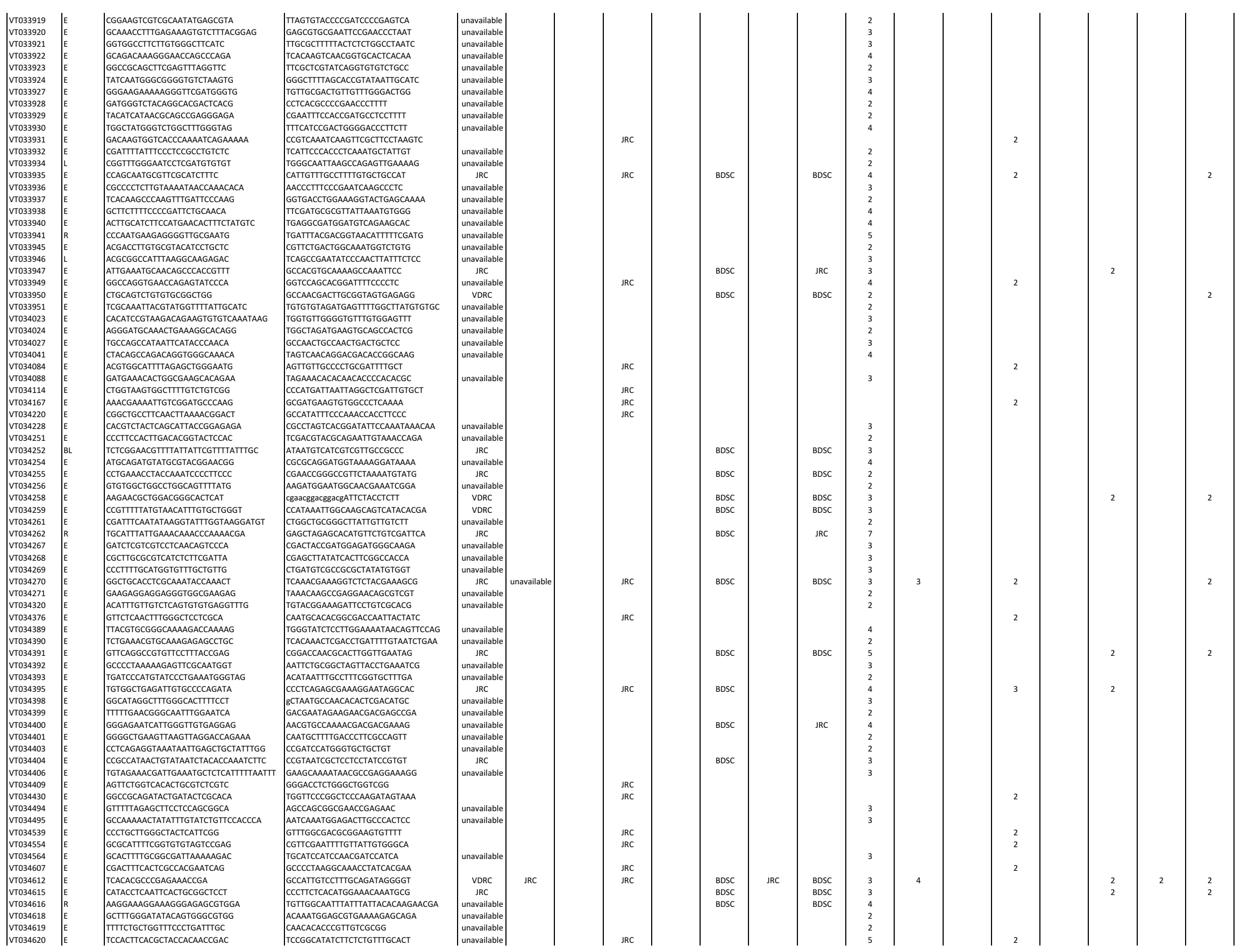




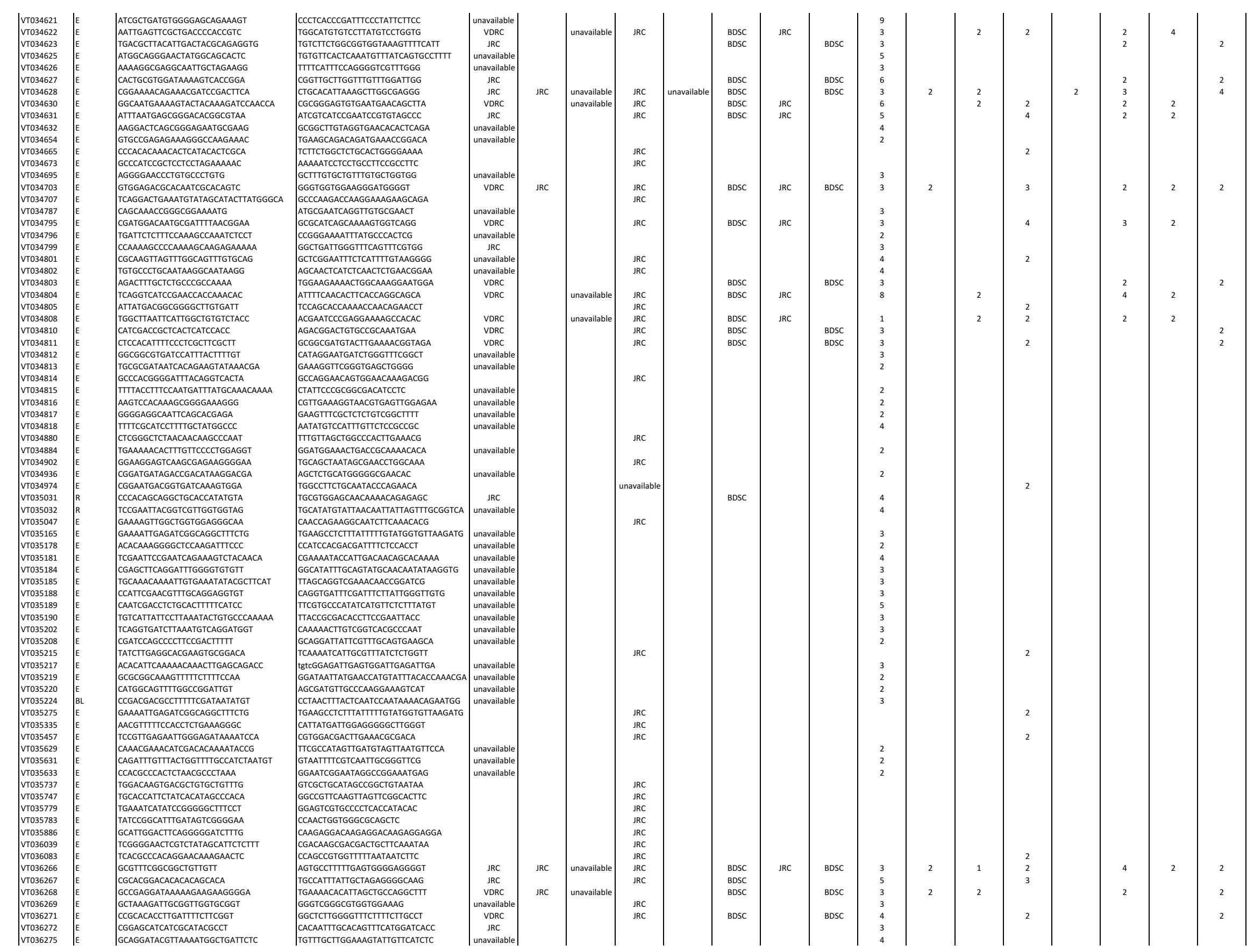




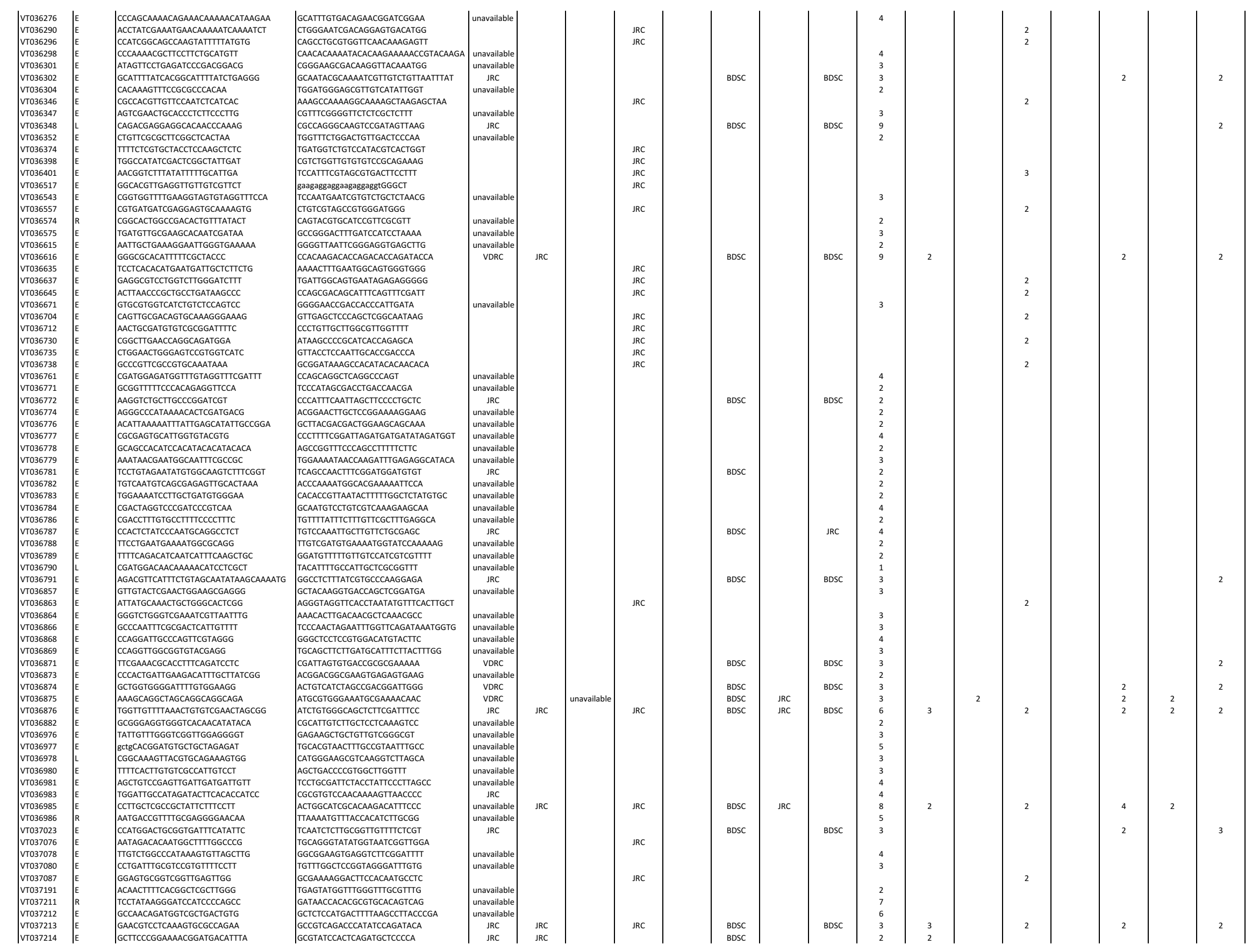




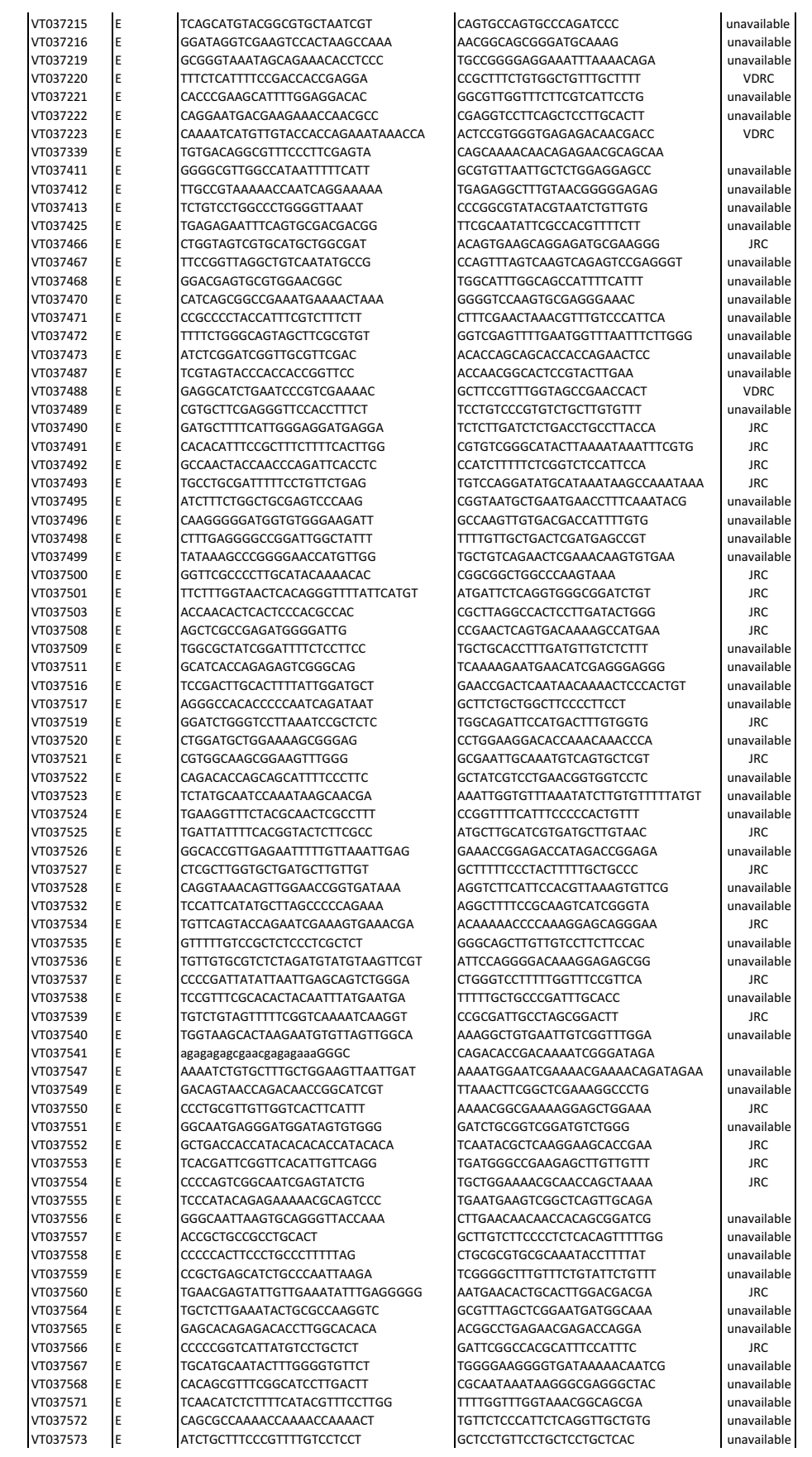

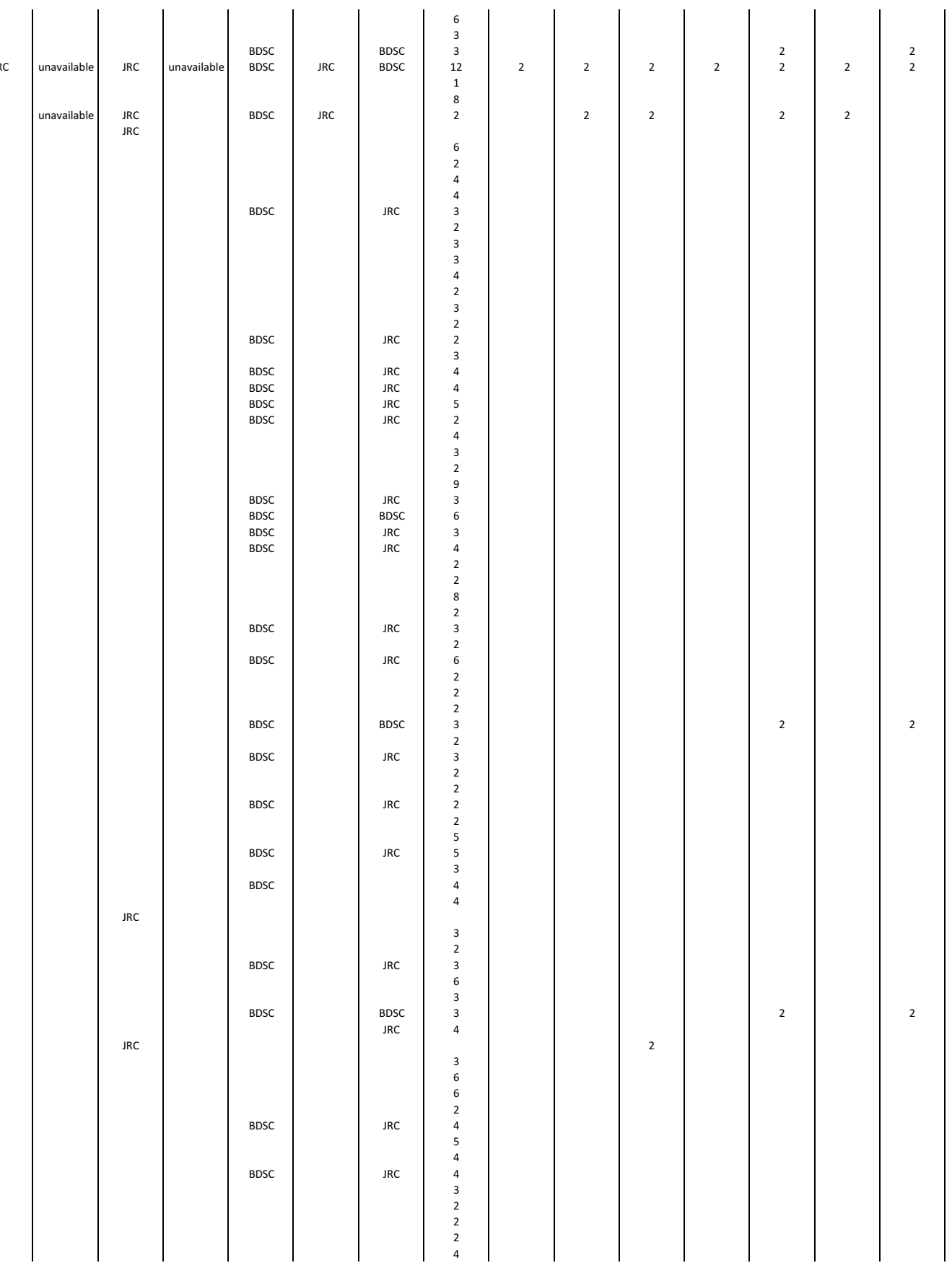




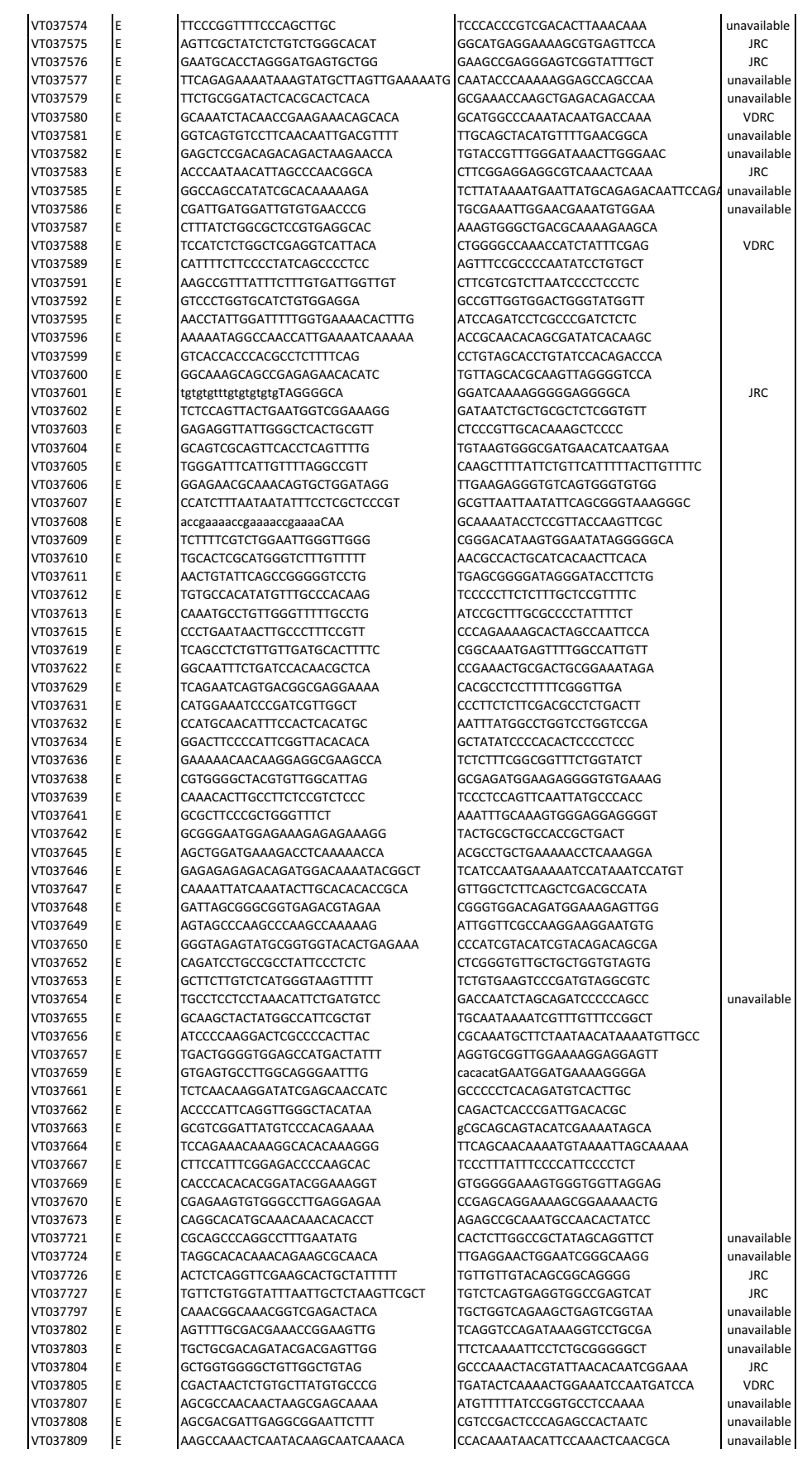

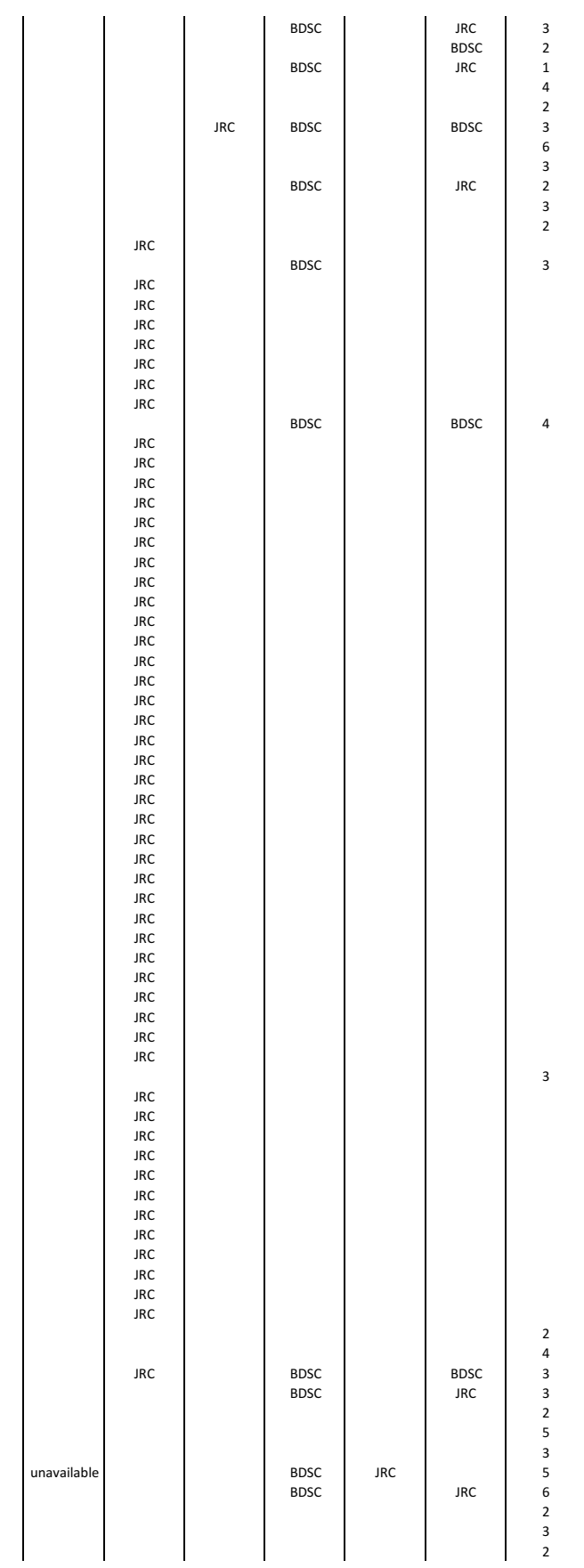




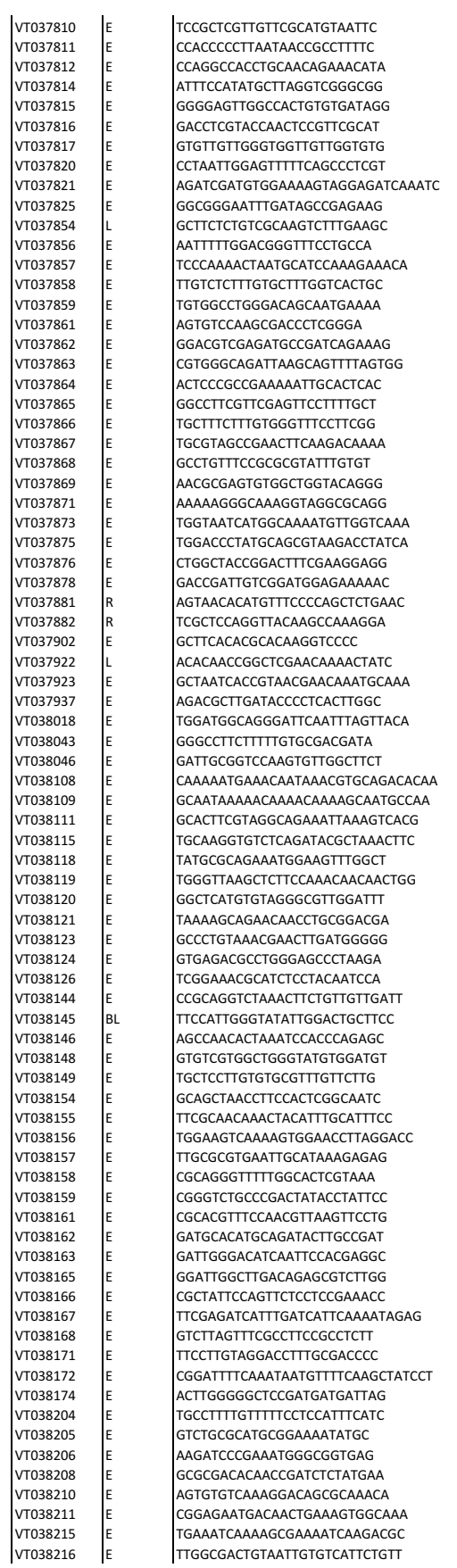

\begin{tabular}{|c|}
\hline 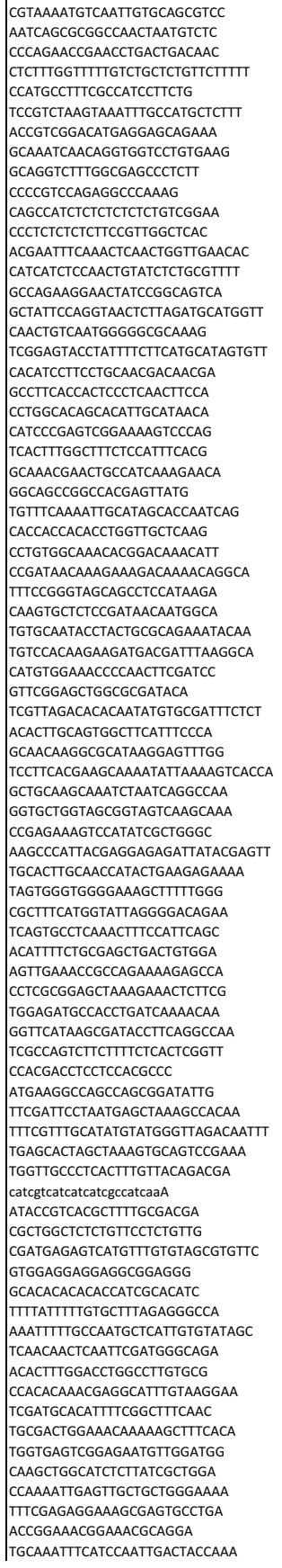 \\
\hline
\end{tabular}
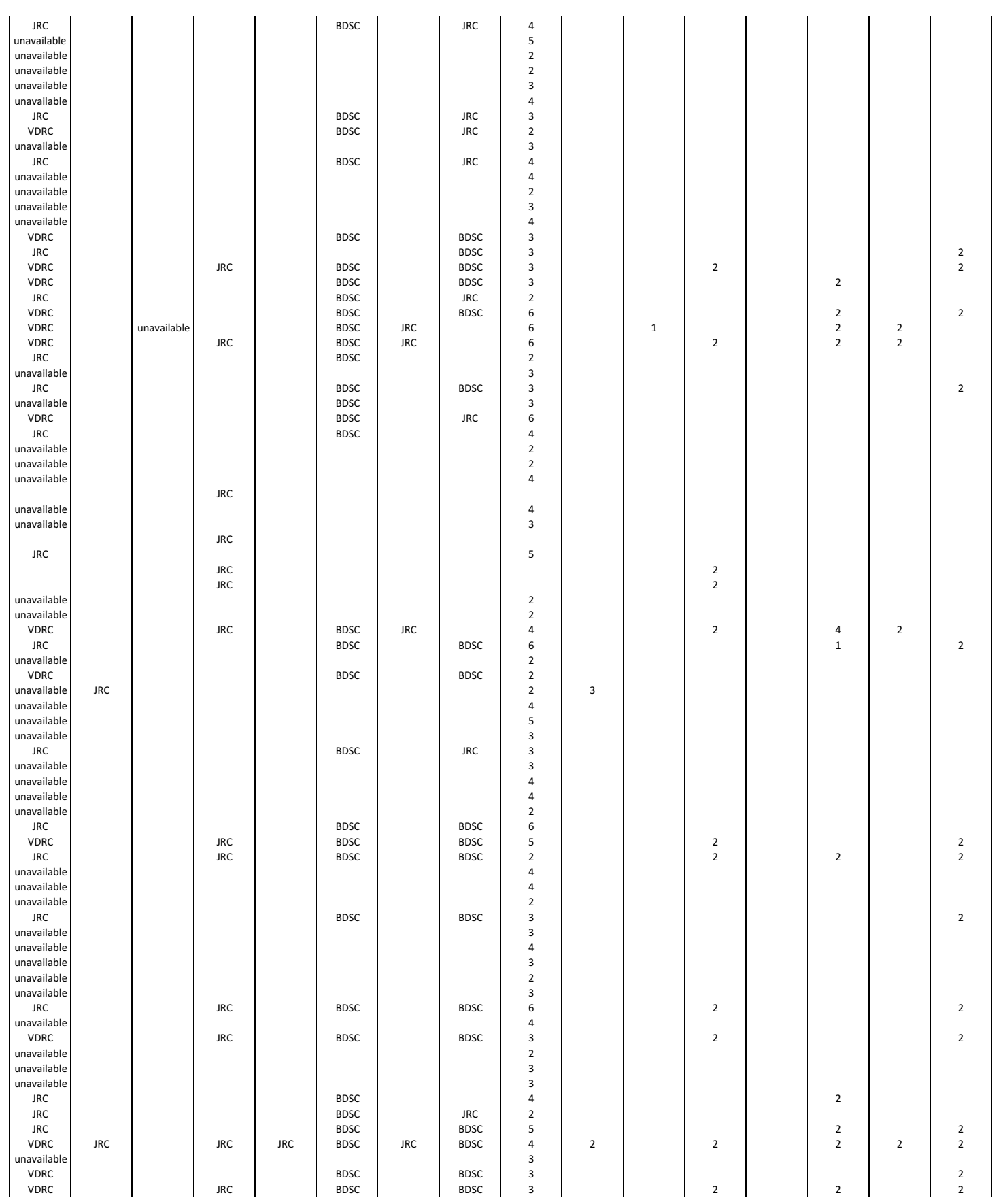


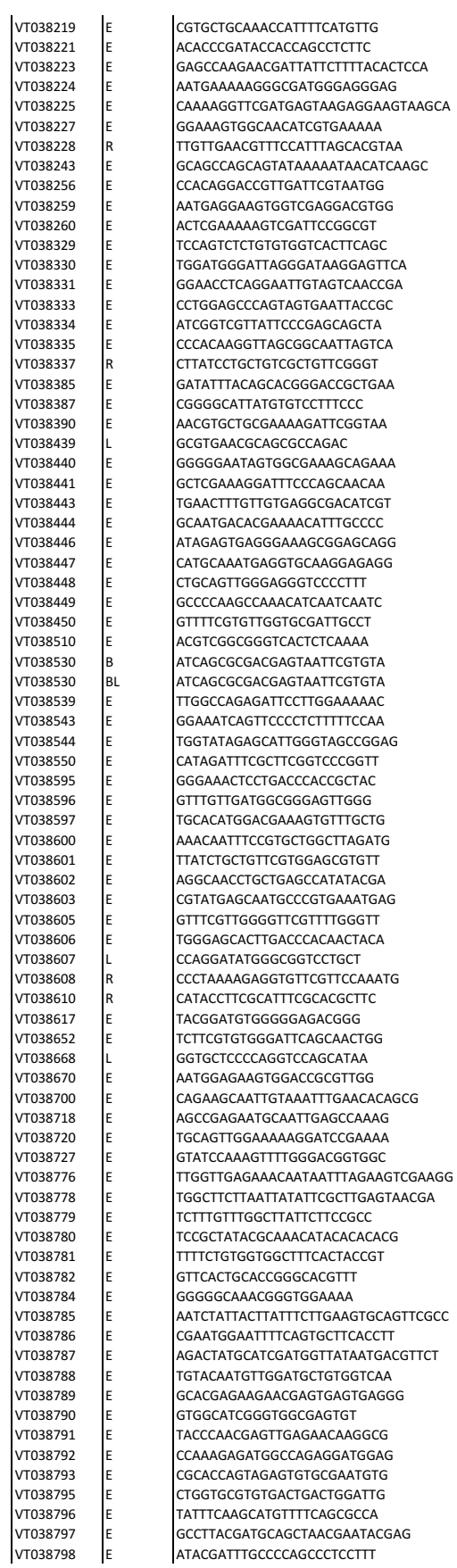

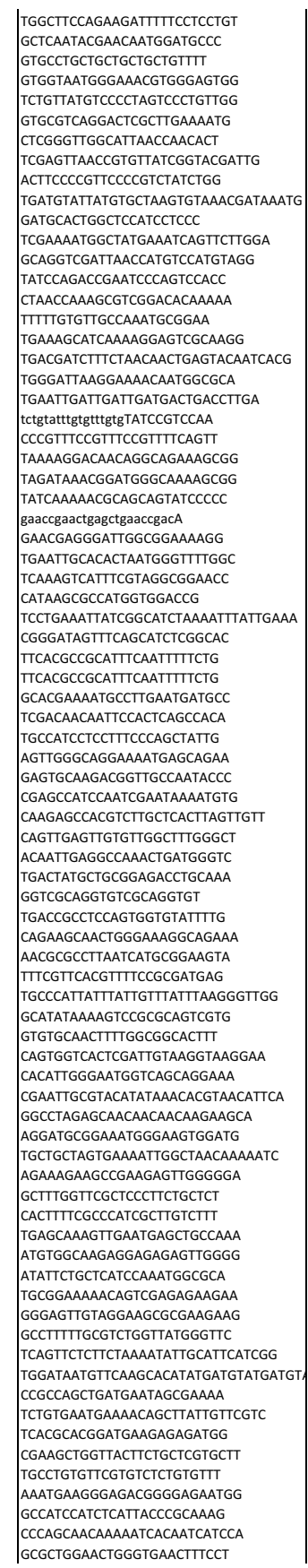
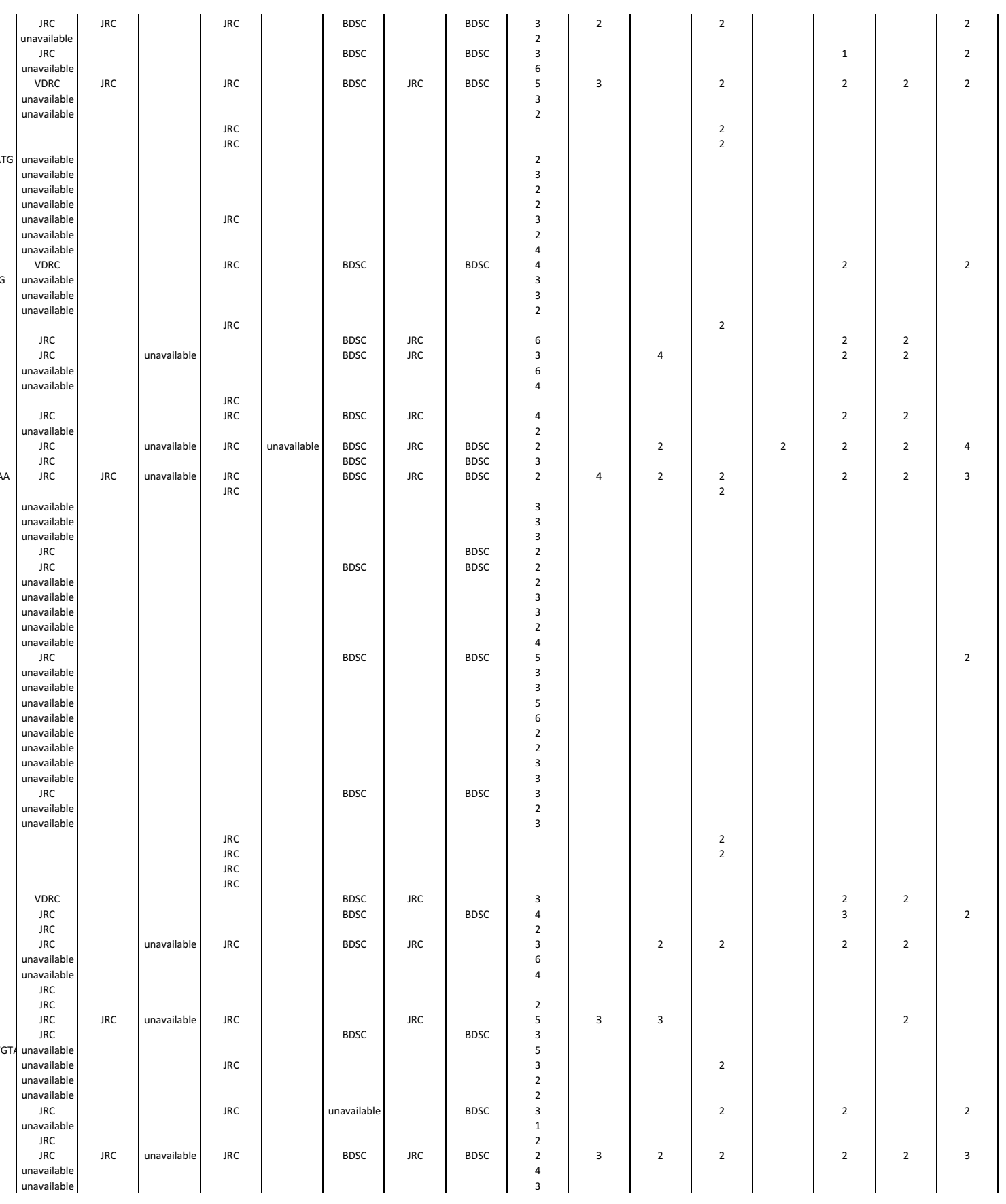


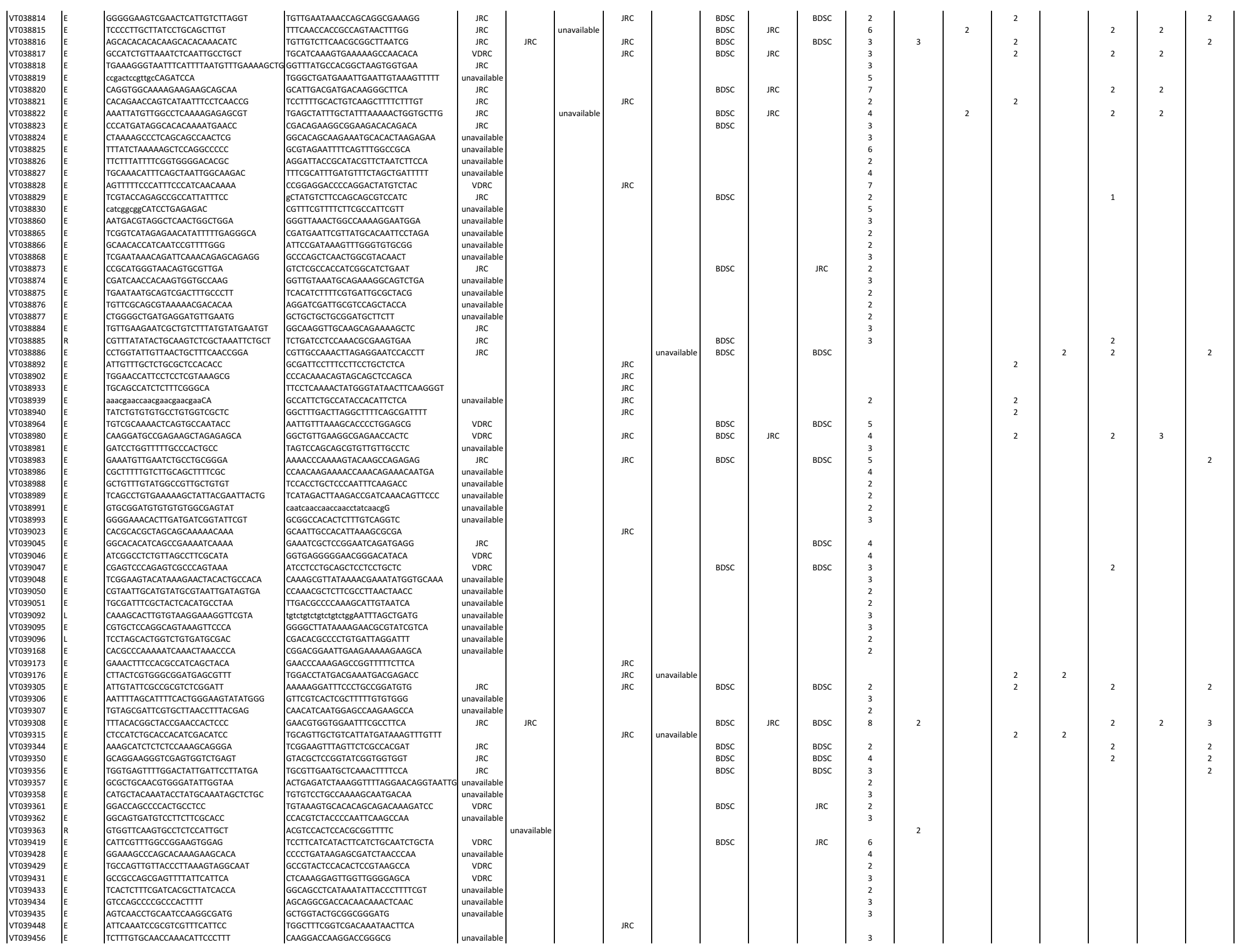




\begin{tabular}{|c|c|c|c|c|}
\hline 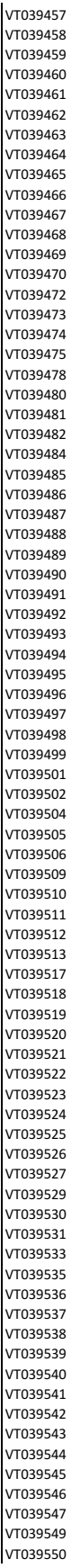 & & 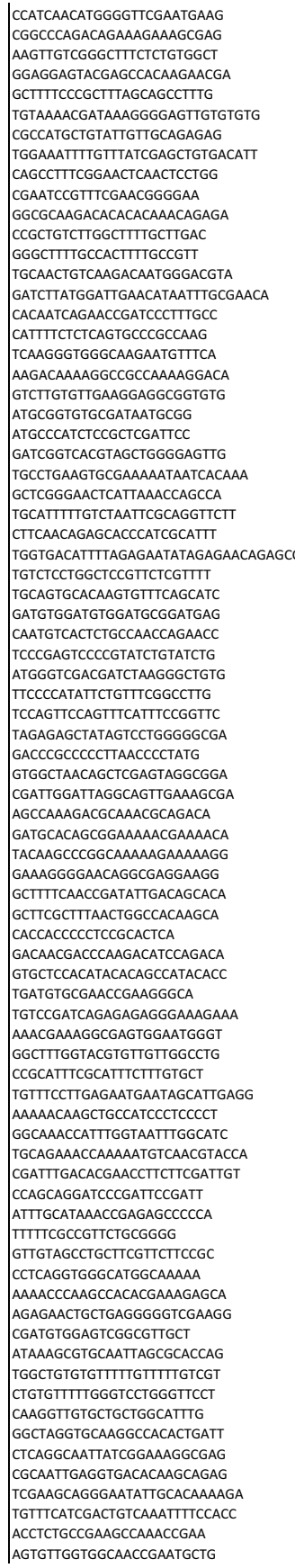 & 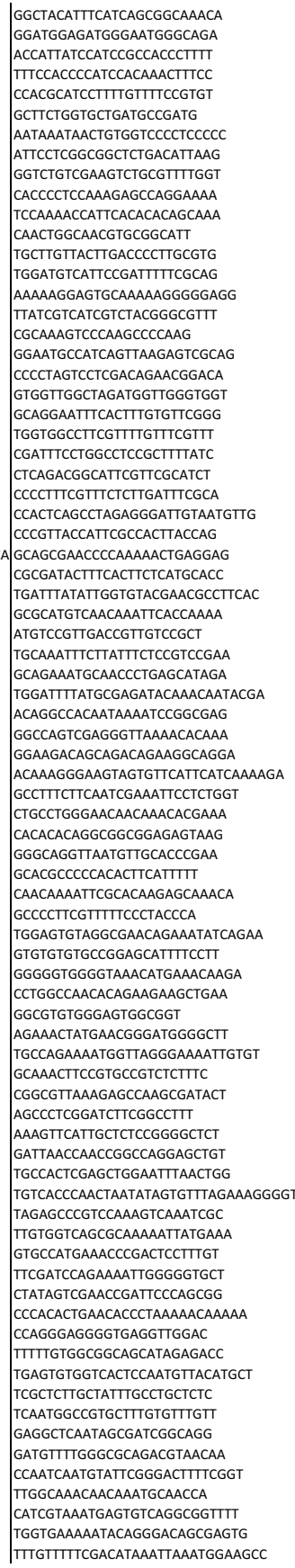 & 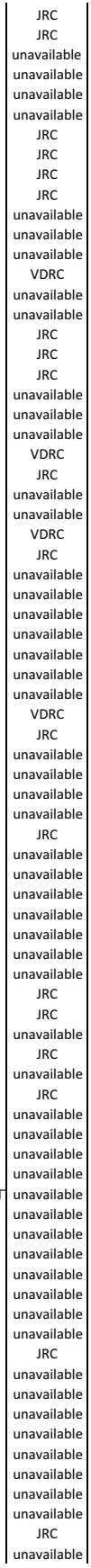 \\
\hline
\end{tabular}
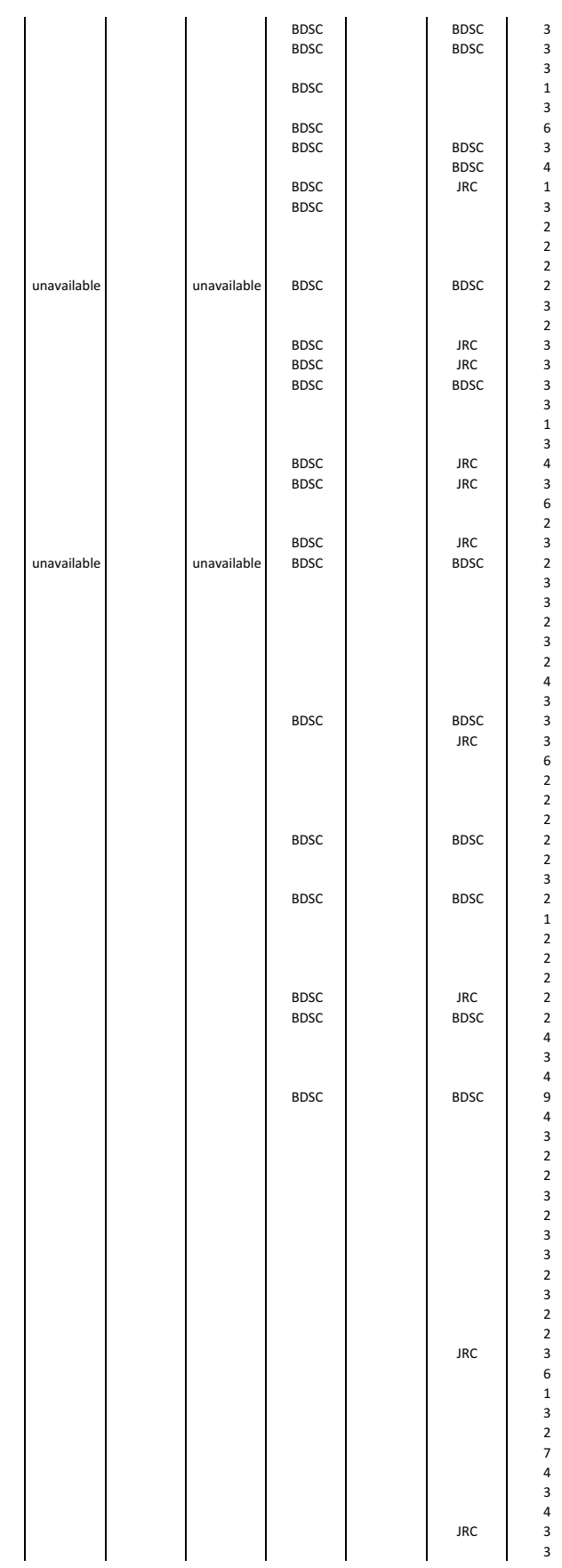

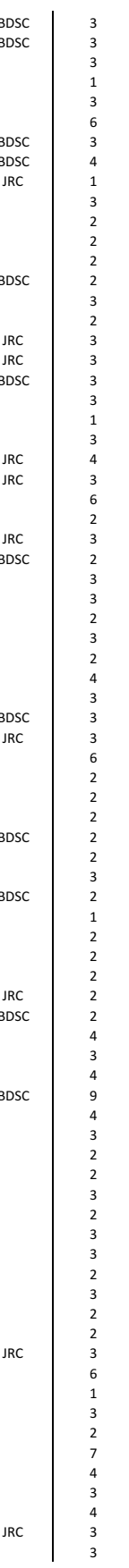




\begin{tabular}{|c|c|c|}
\hline 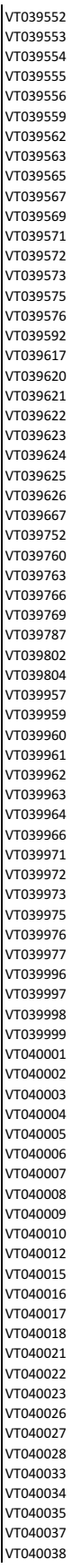 & & 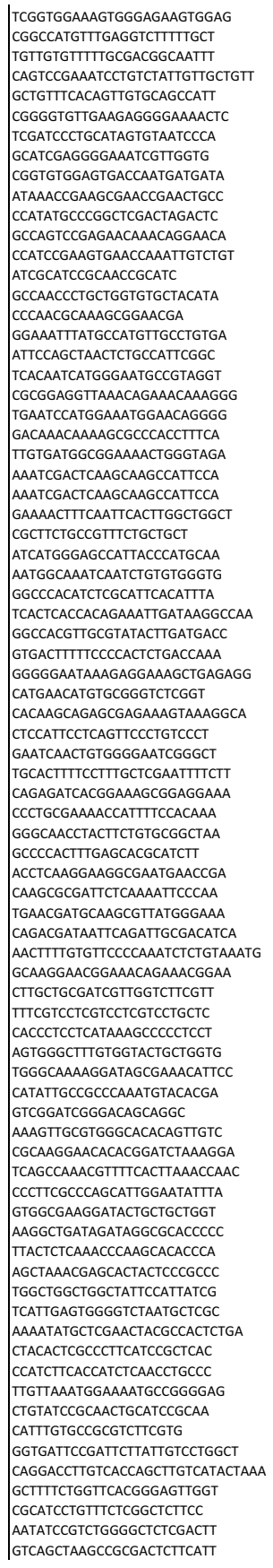 \\
\hline
\end{tabular}
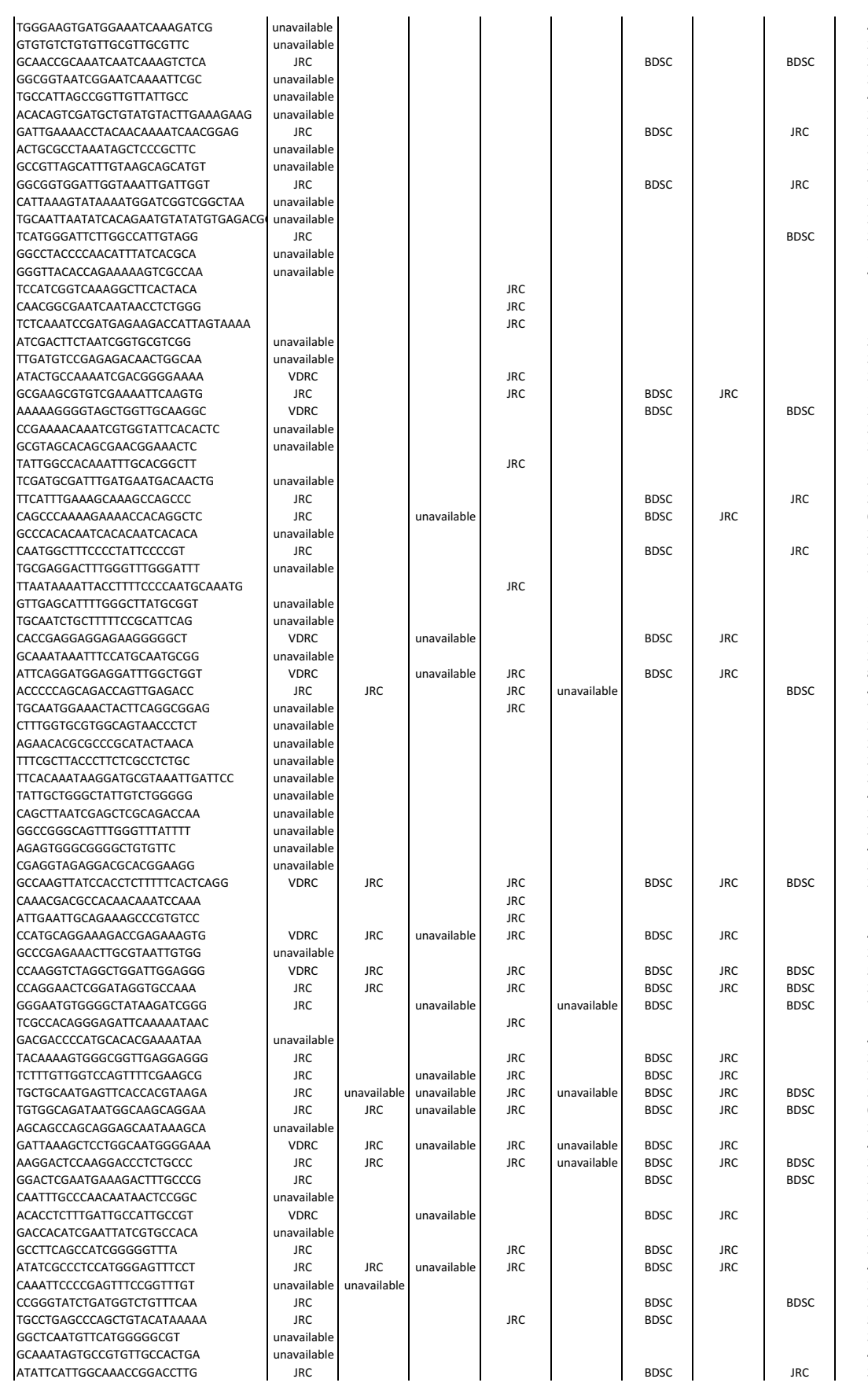


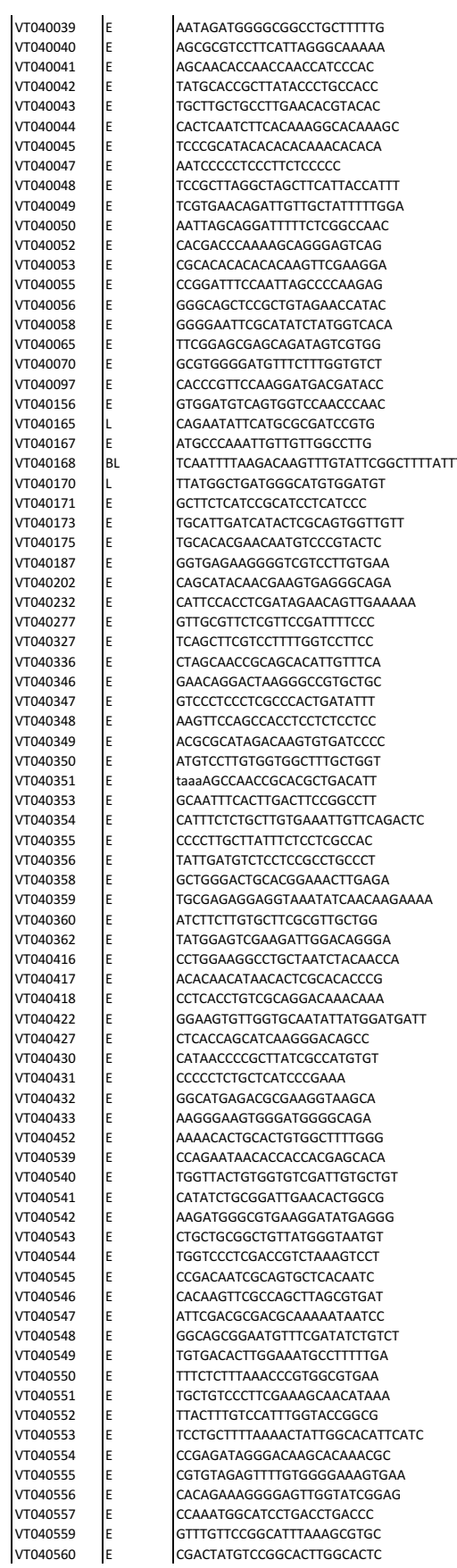

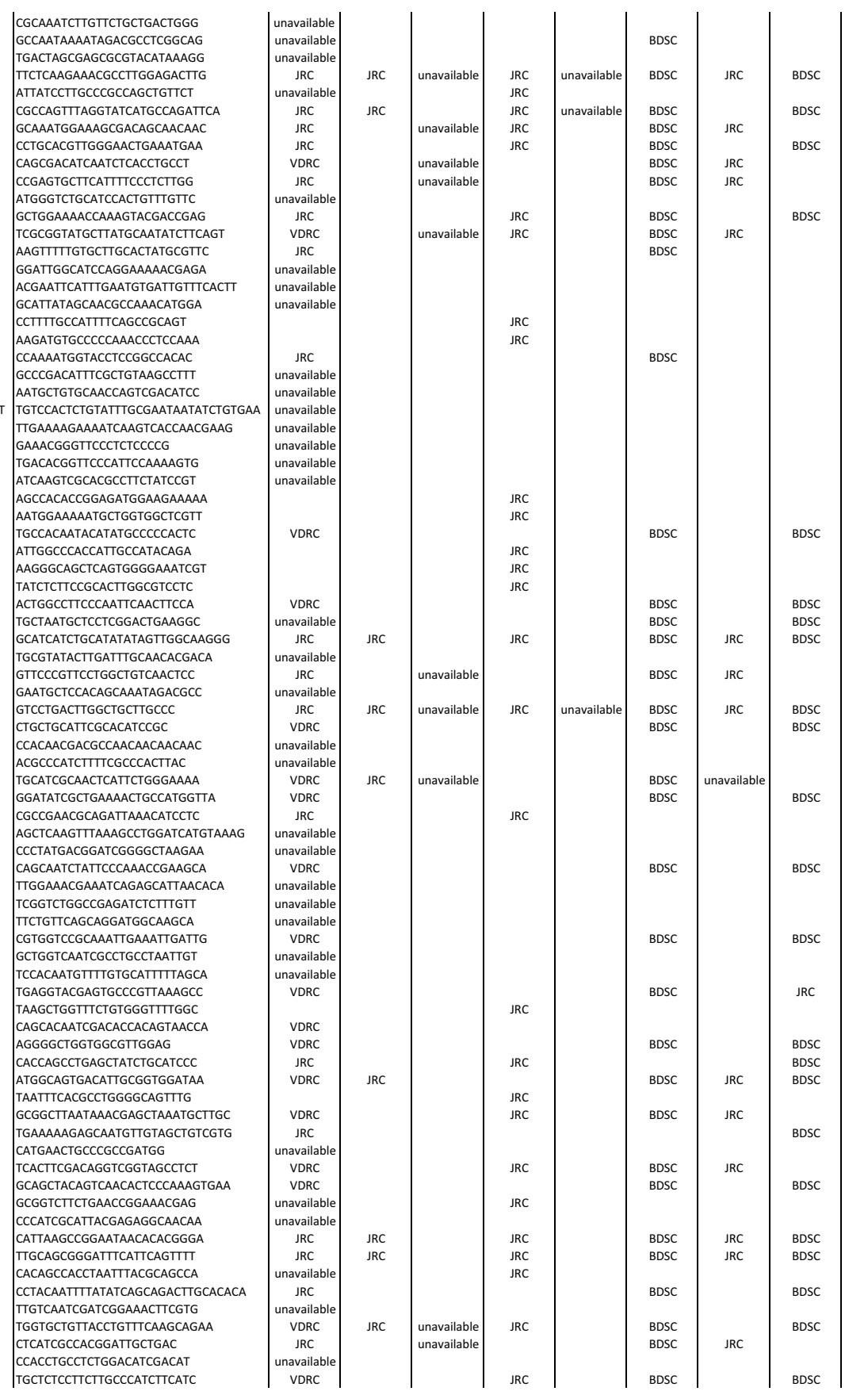

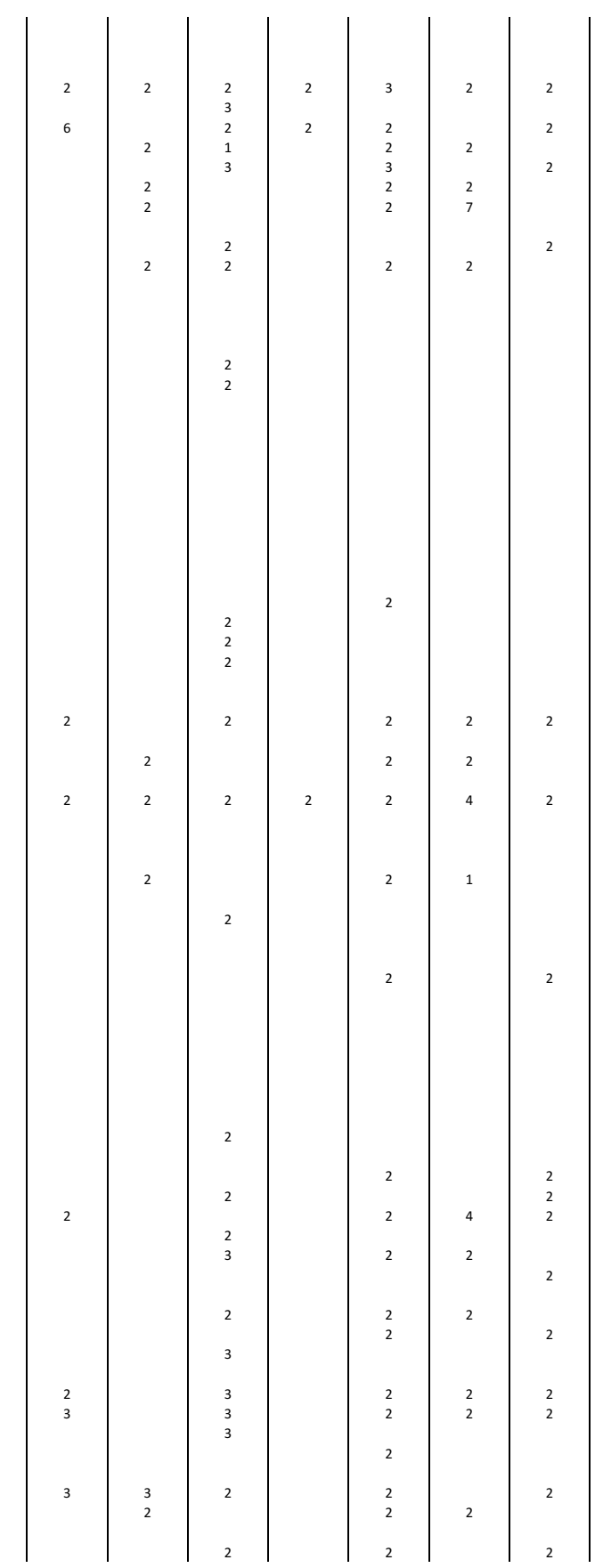



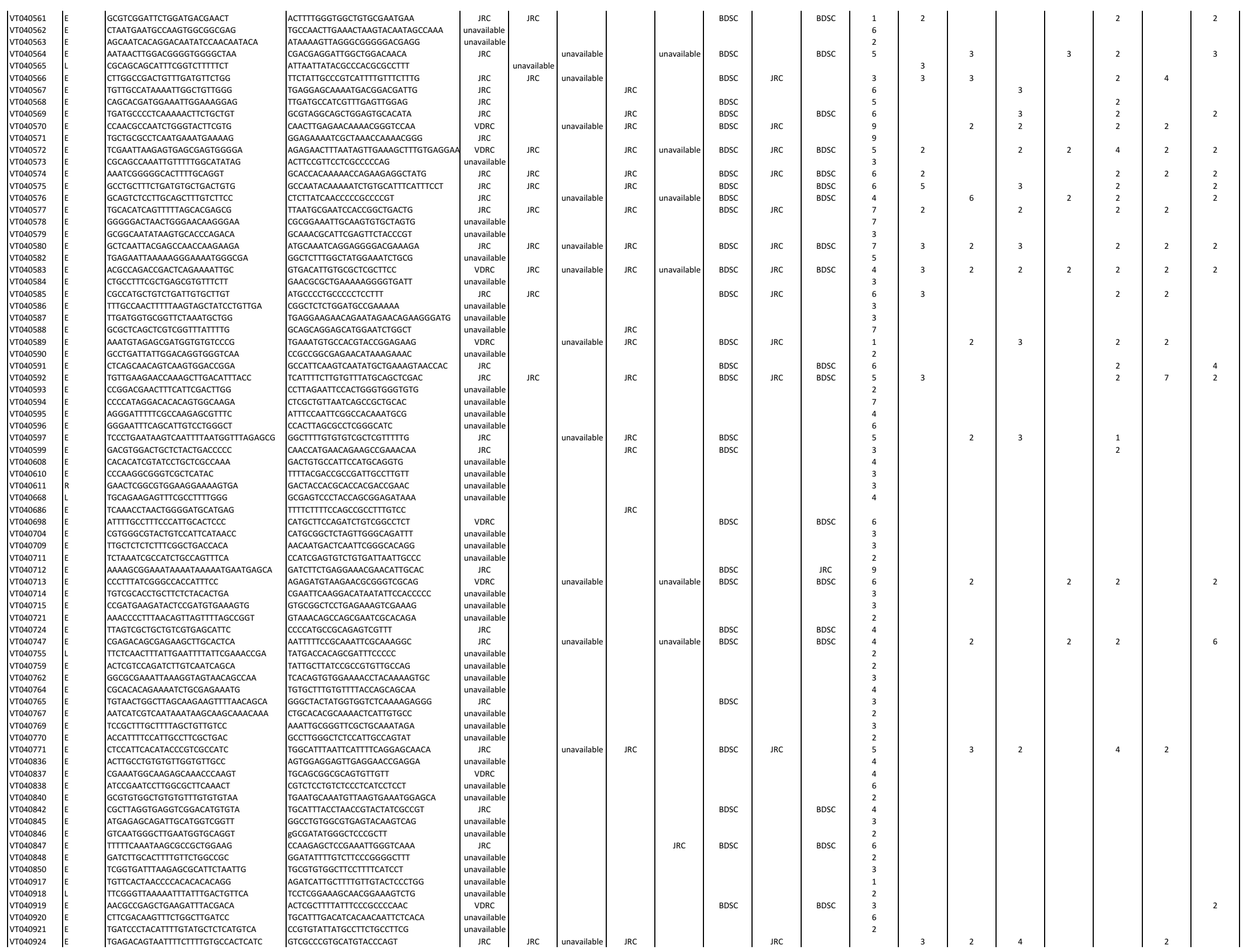


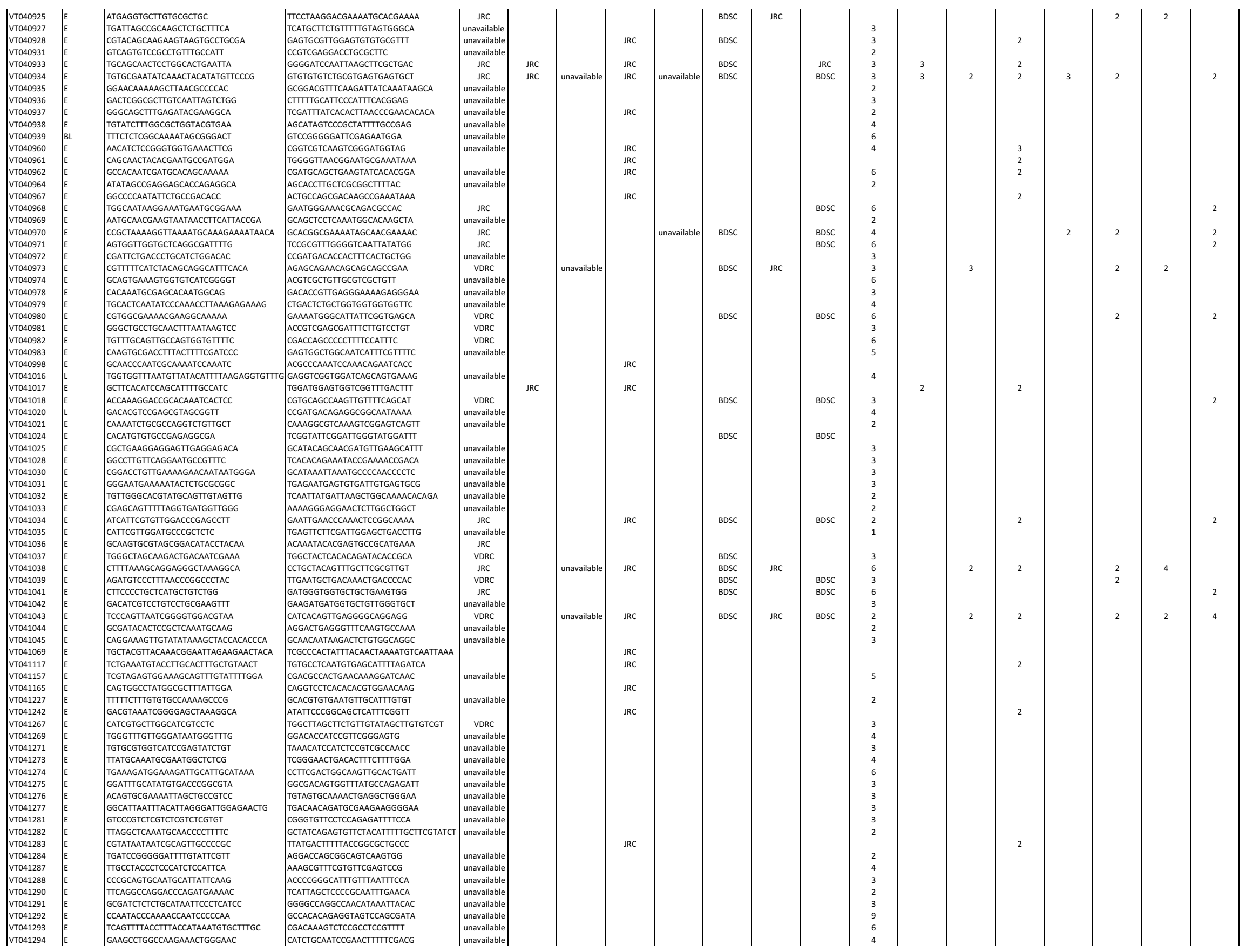




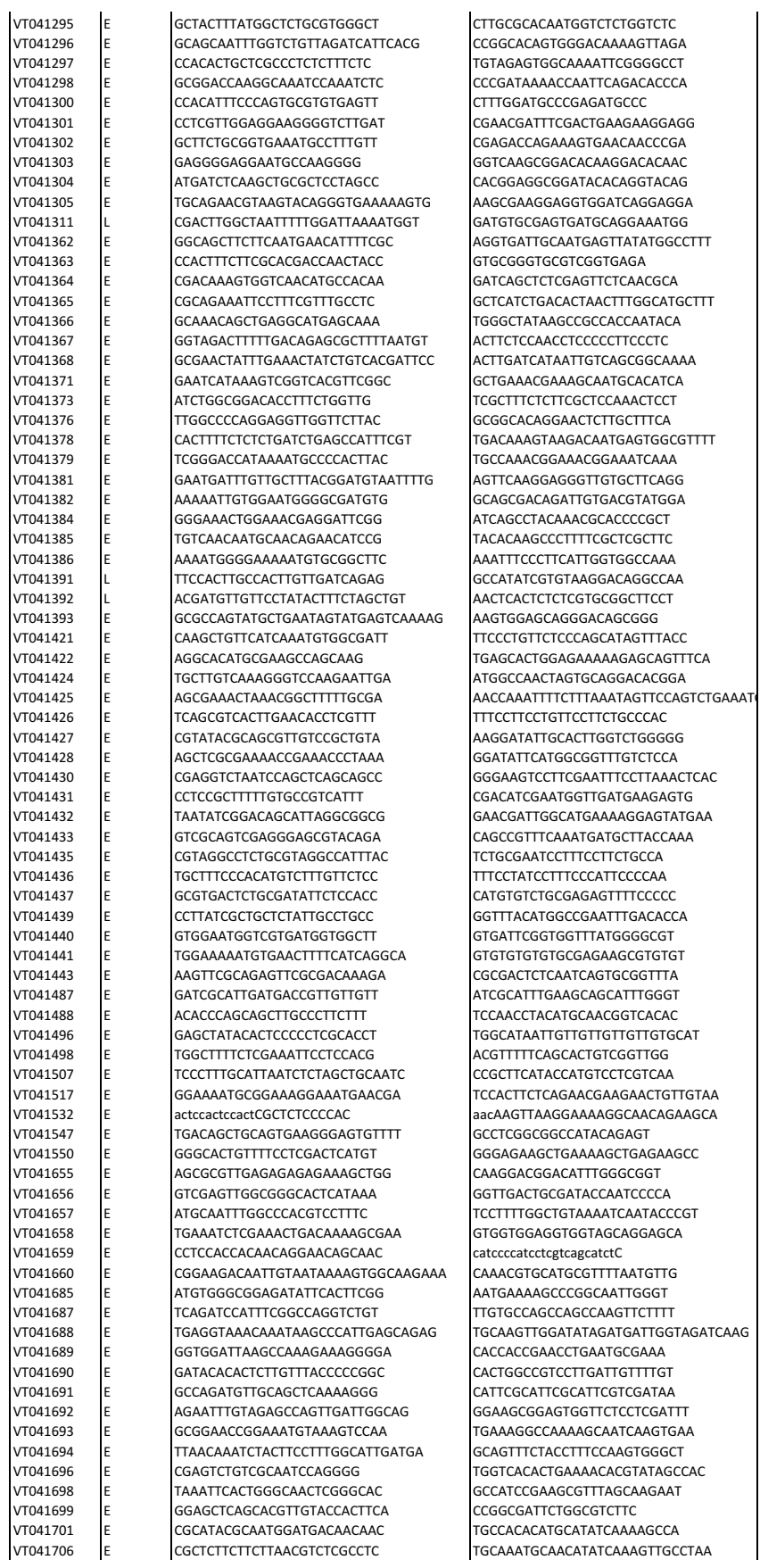

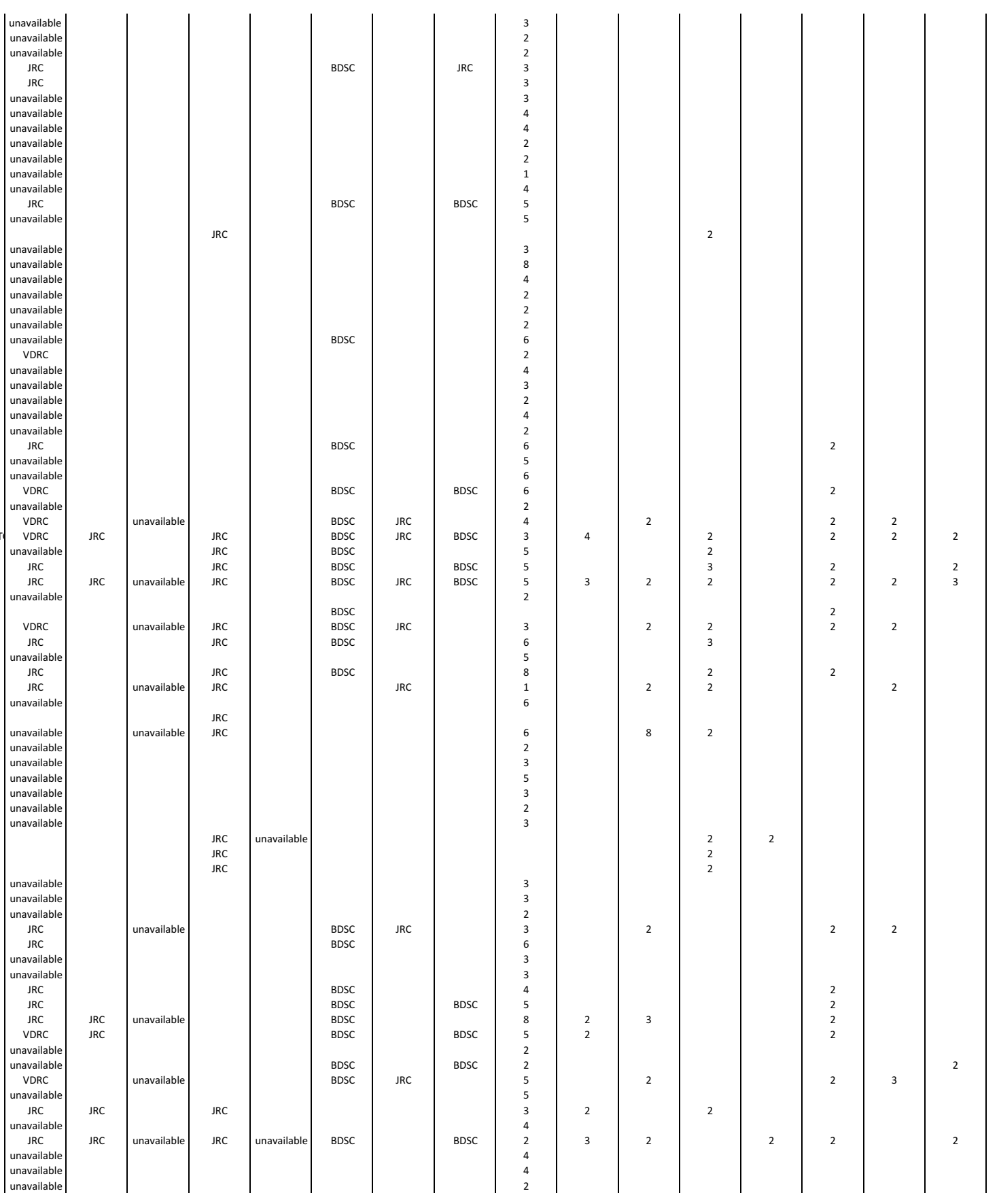




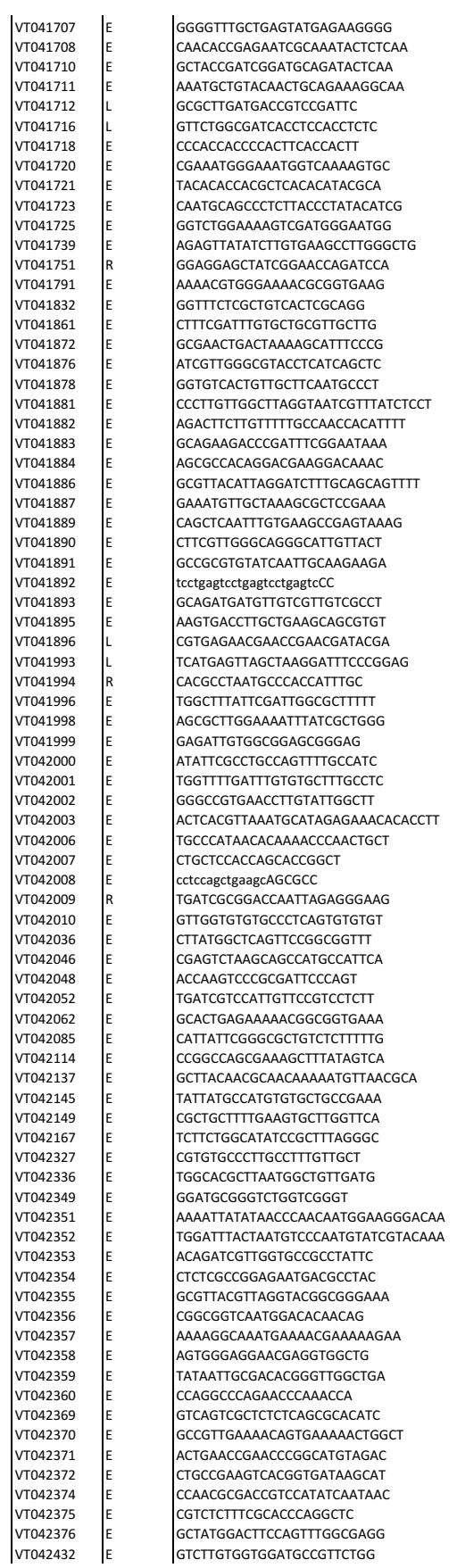

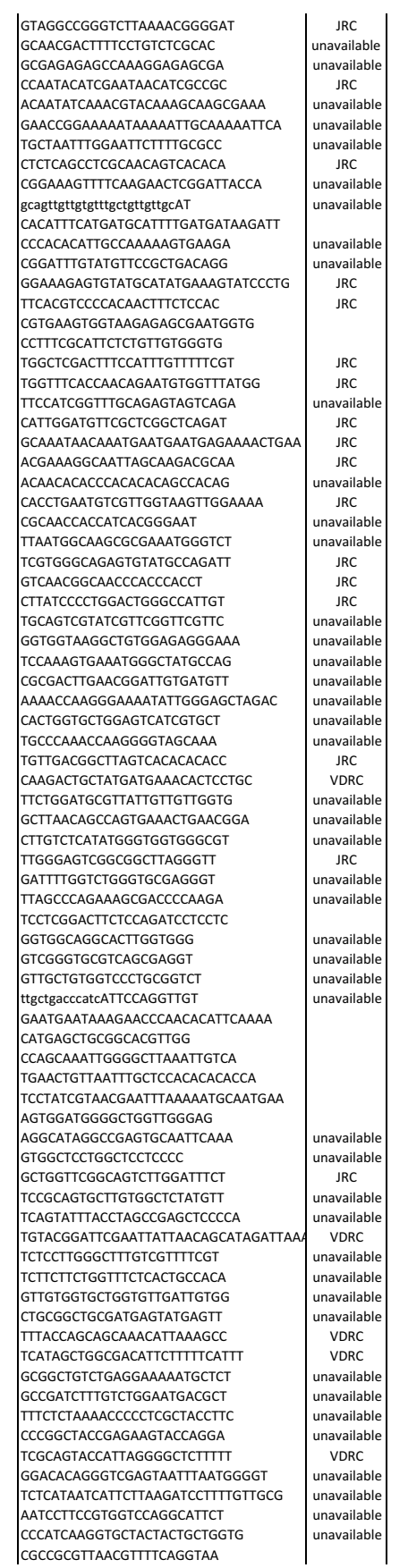
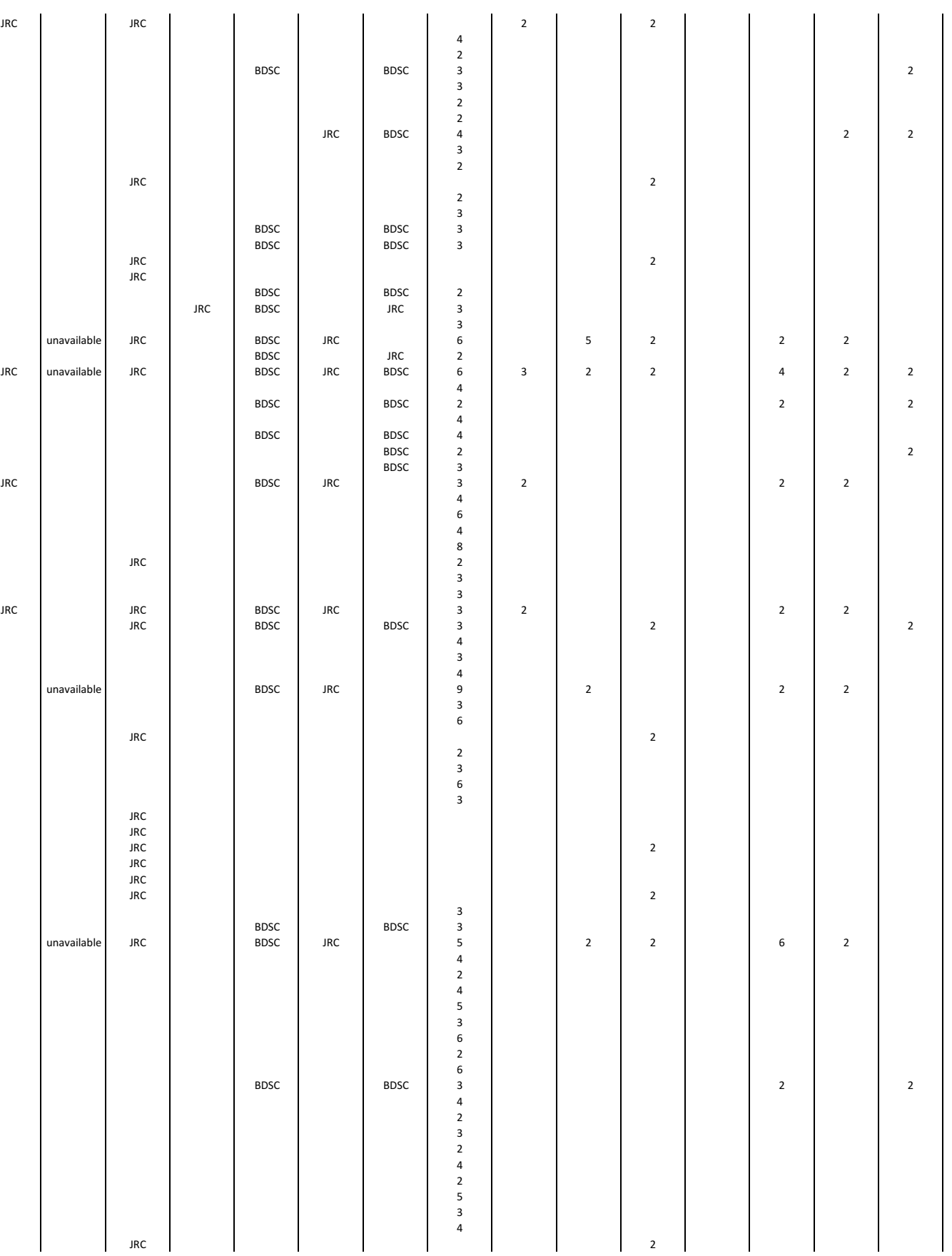


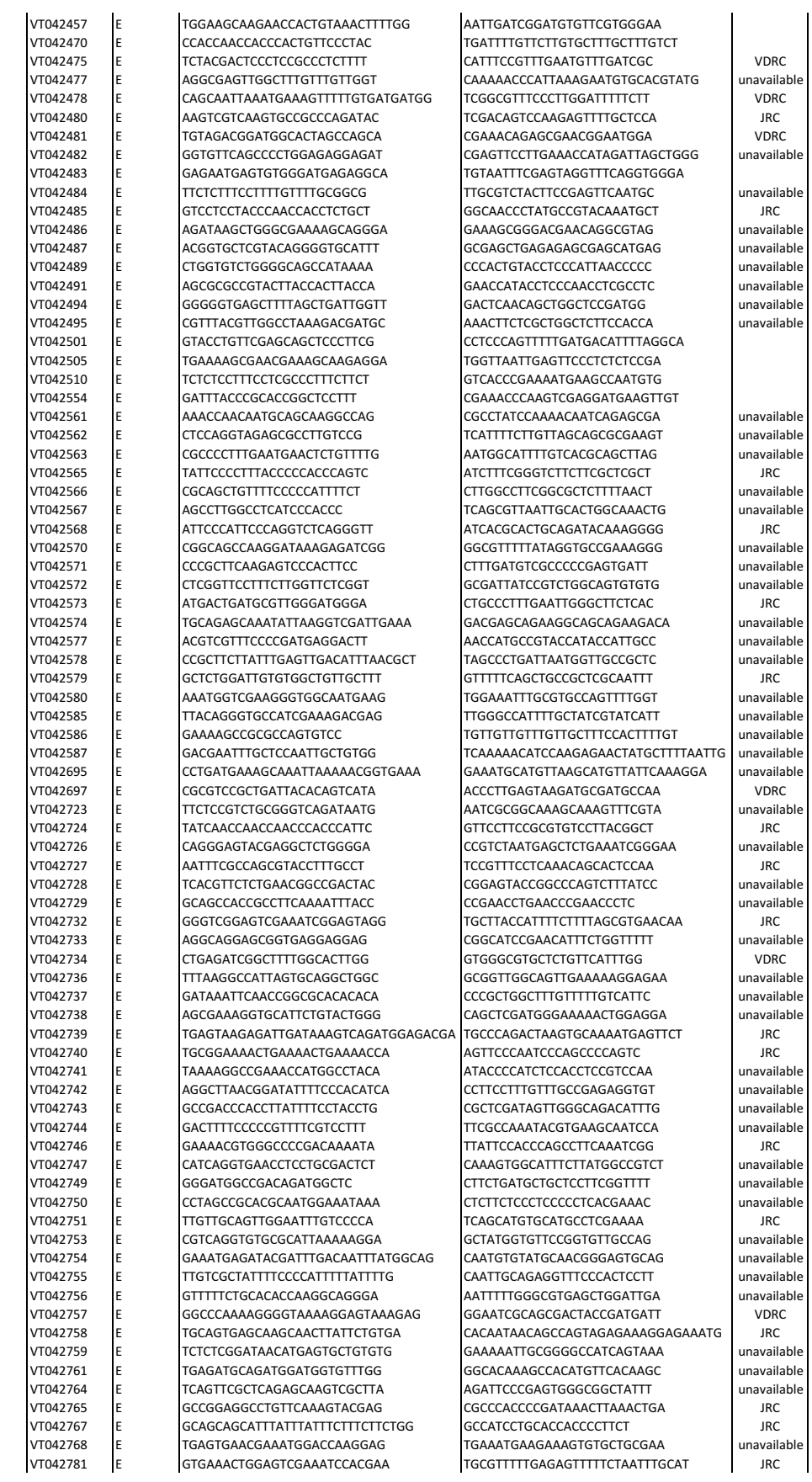

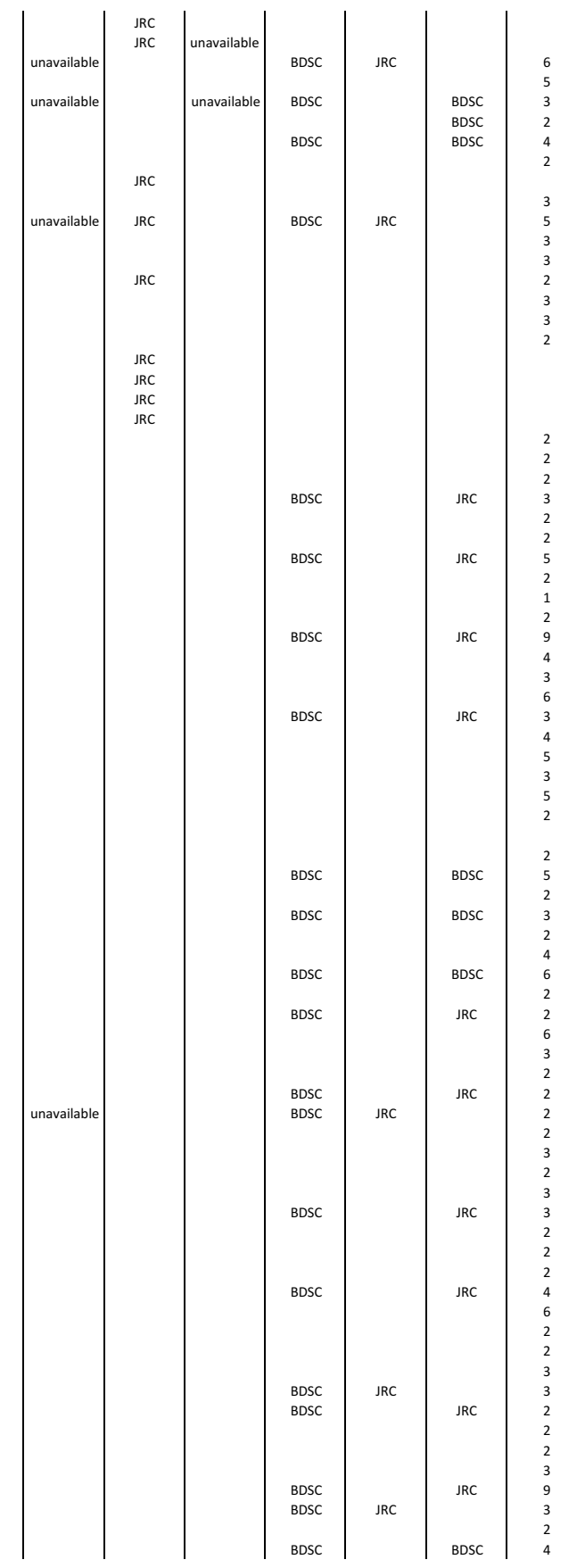




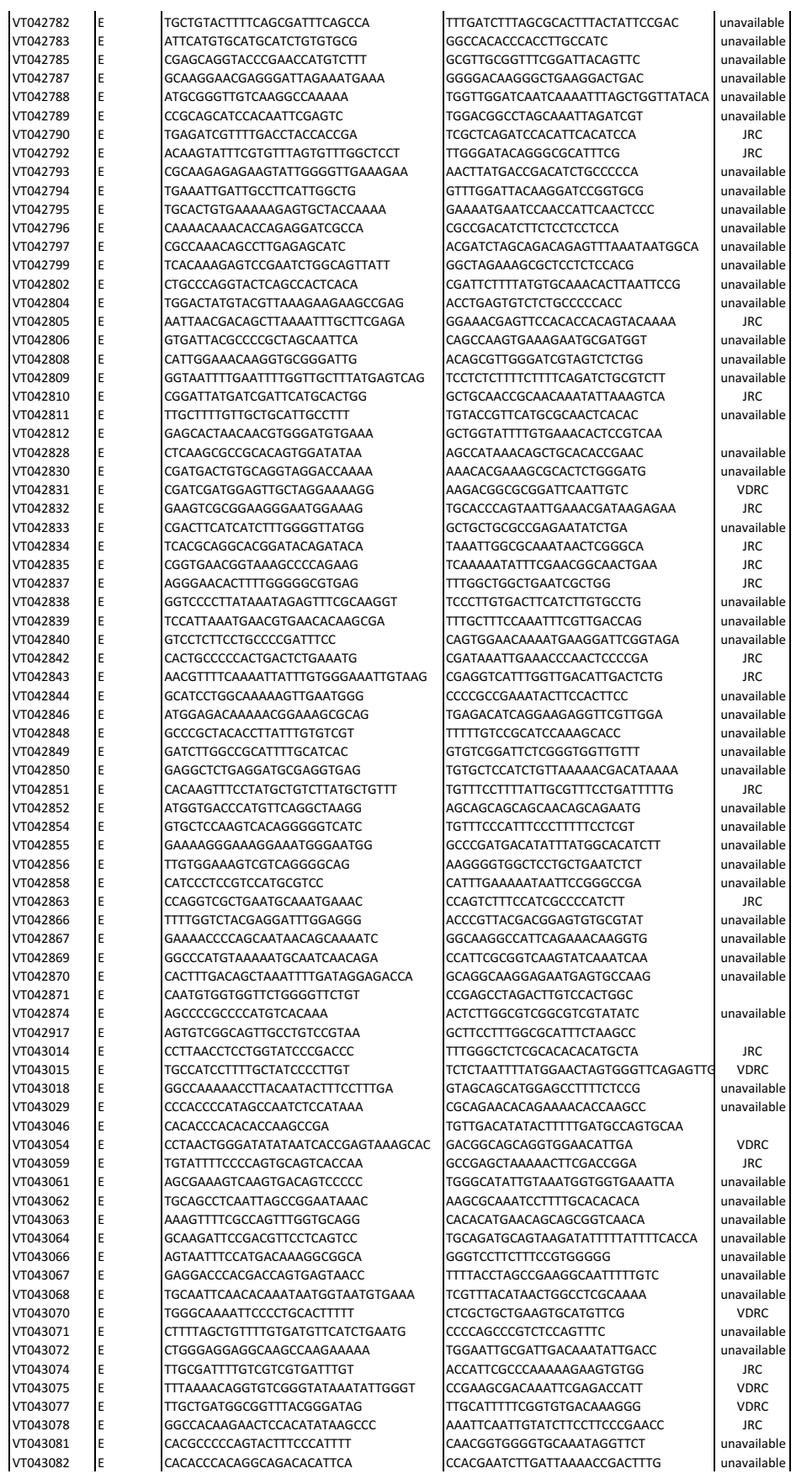
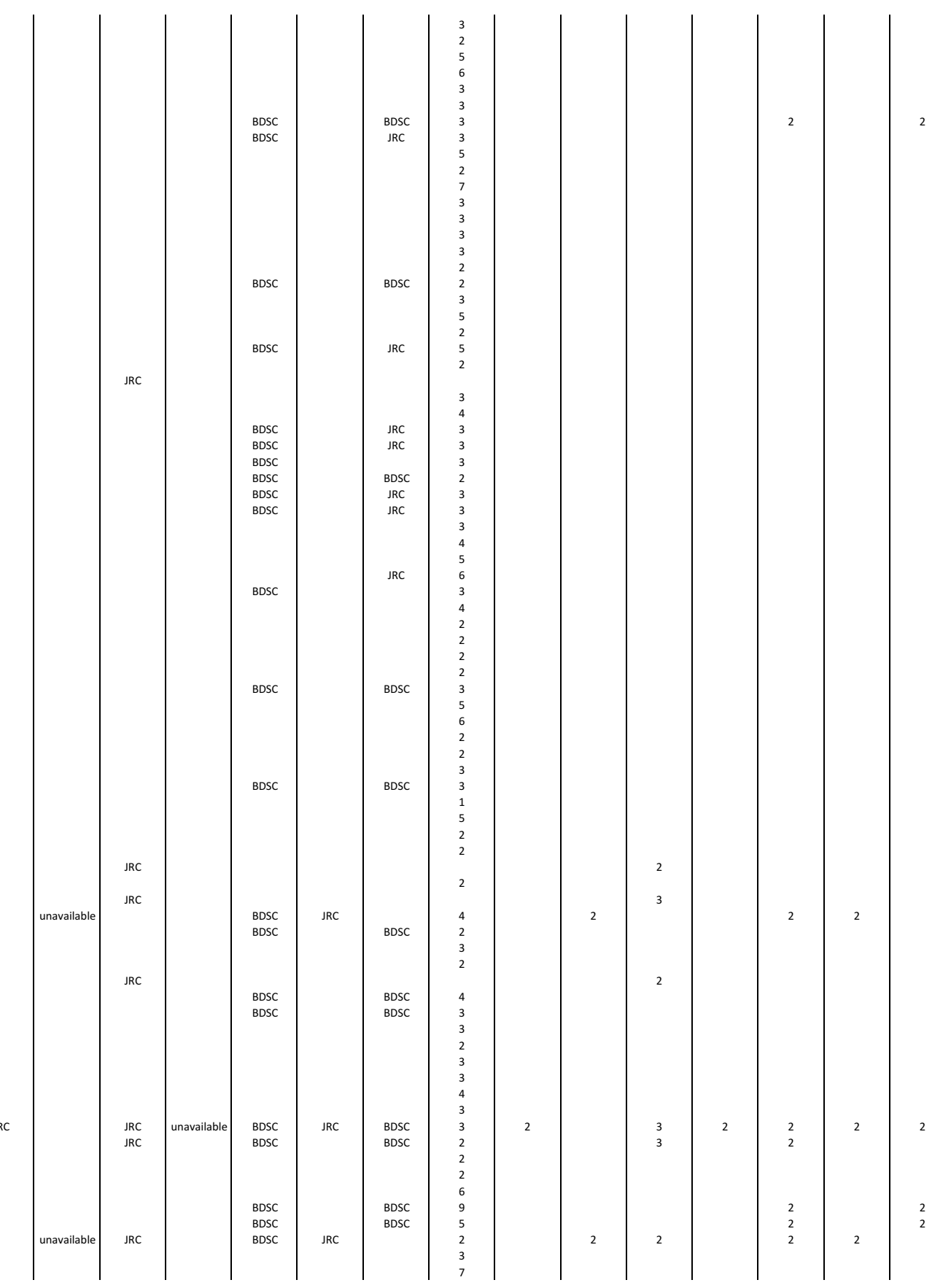


\begin{tabular}{|c|c|c|}
\hline \multicolumn{2}{|c|}{ 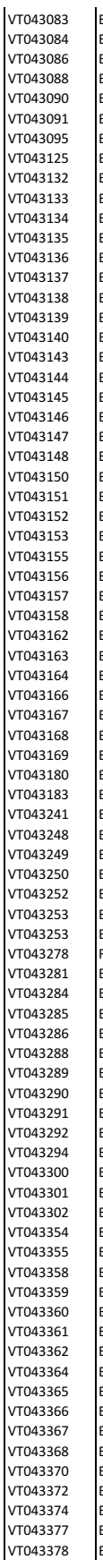 } & 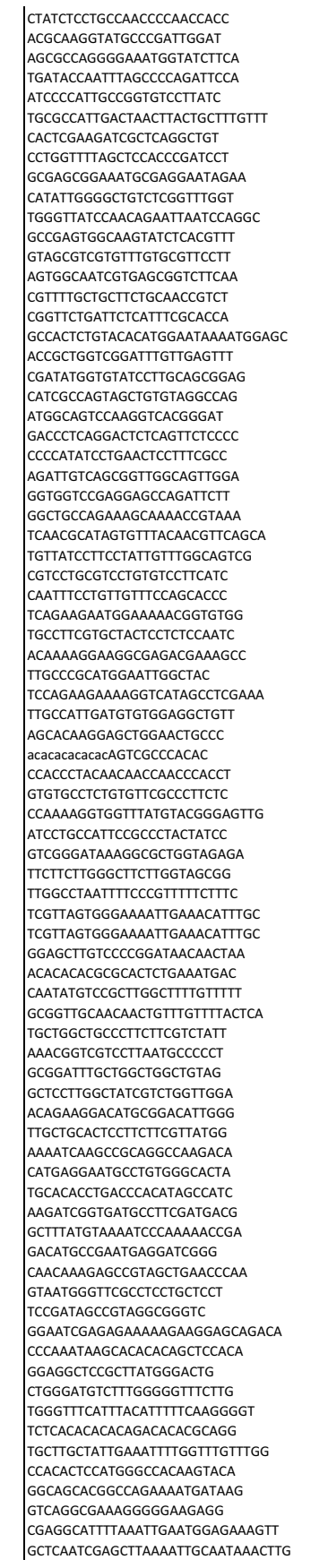 \\
\hline
\end{tabular}

\begin{tabular}{|c|c|c|c|c|c|}
\hline 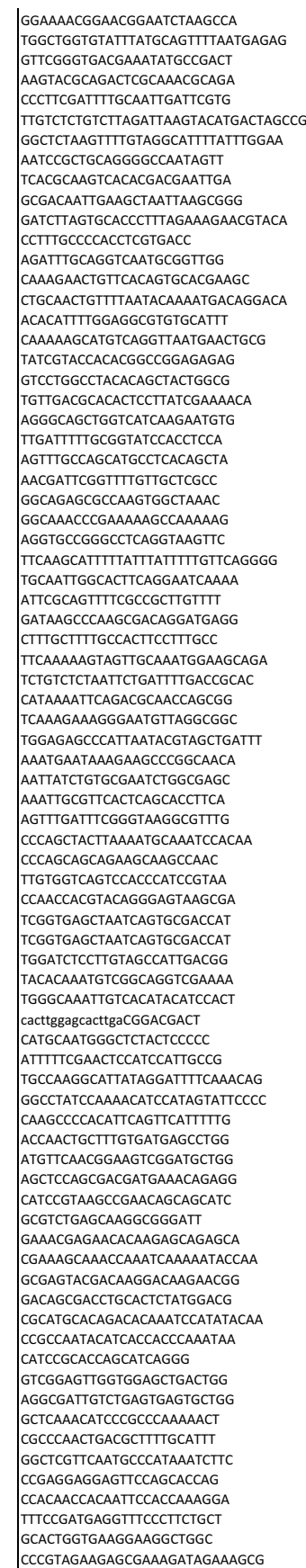 & 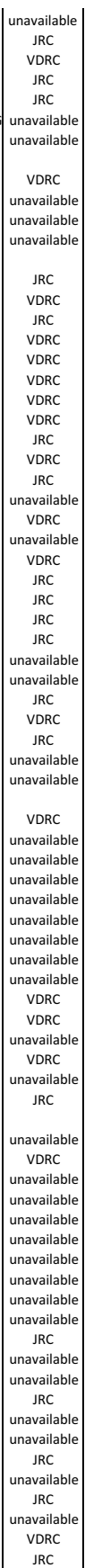 & $\begin{array}{l}\mathrm{JRC} \\
\mathrm{JRC}\end{array}$ & $\mid$\begin{tabular}{l|} 
unavailable \\
unavailable
\end{tabular} & 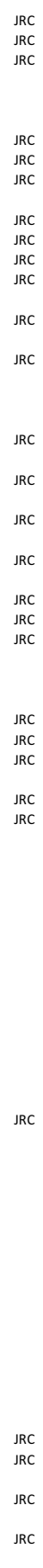 & unavailable \\
\hline
\end{tabular}

\begin{tabular}{|c|c|c|}
\hline $\begin{array}{l}\text { BDSC } \\
\text { BDSC }\end{array}$ & & $\begin{array}{l}\text { BDSC } \\
\text { BDSC }\end{array}$ \\
\hline BDSC & ${ }_{J R C}$ & $\begin{array}{l}\text { BRL } \\
\text { BDS }\end{array}$ \\
\hline BDSC & ${ }^{\mathrm{JRC}}$ & \\
\hline BDSC & & \\
\hline $\begin{array}{l}\text { BDSC } \\
\text { BDSC }\end{array}$ & $\mathrm{JRC}$ & BDSC \\
\hline $\begin{array}{l}\text { DOD } \\
\text { BDSC }\end{array}$ & & $\begin{array}{l}\text { DDSSC } \\
\text { BDS }\end{array}$ \\
\hline BDSC & JRC & \\
\hline $\begin{array}{l}\text { BDSC } \\
\text { BDS }\end{array}$ & JRC & $\begin{array}{l}\text { BDSC } \\
B D S C\end{array}$ \\
\hline BDSC & & \\
\hline BDSC & & BDSC \\
\hline 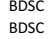 & & BDSC \\
\hline BDSC & $\mathrm{JRC}$ & \\
\hline BDSC & $\mathrm{JRC}$ & BDSC \\
\hline $\begin{array}{l}\text { BDSC } \\
\text { BDSC }\end{array}$ & $J B C$ & \\
\hline BDSC & JRC & \\
\hline $\begin{array}{l}\text { BDSC } \\
\text { BDSC }\end{array}$ & JRC & BDSC \\
\hline BDSC & JRC & BDSC \\
\hline $\begin{array}{l}\text { BDSC } \\
\text { BDSC }\end{array}$ & & BDSC \\
\hline $\begin{array}{l}\text { BUD } \\
\text { BDS }\end{array}$ & $\begin{array}{l}\mathrm{KC} \\
\mathrm{JRC}\end{array}$ & \\
\hline BDSC & & BDSC \\
\hline $\begin{array}{l}\text { BDSC } \\
\text { BDSC }\end{array}$ & & BDSC \\
\hline BDSC & & BDSC \\
\hline BDSC & & \\
\hline BDSC & & BDSC \\
\hline BDSC & $\mathrm{JRC}$ & \\
\hline BDSC & ${ }_{\mathrm{JRC}}$ & \\
\hline BDSC & & BDSC \\
\hline $\begin{array}{l}\text { BDSC } \\
\text { BDSC }\end{array}$ & & $\begin{array}{l}\text { BDSC } \\
\text { BDSC }\end{array}$ \\
\hline
\end{tabular}

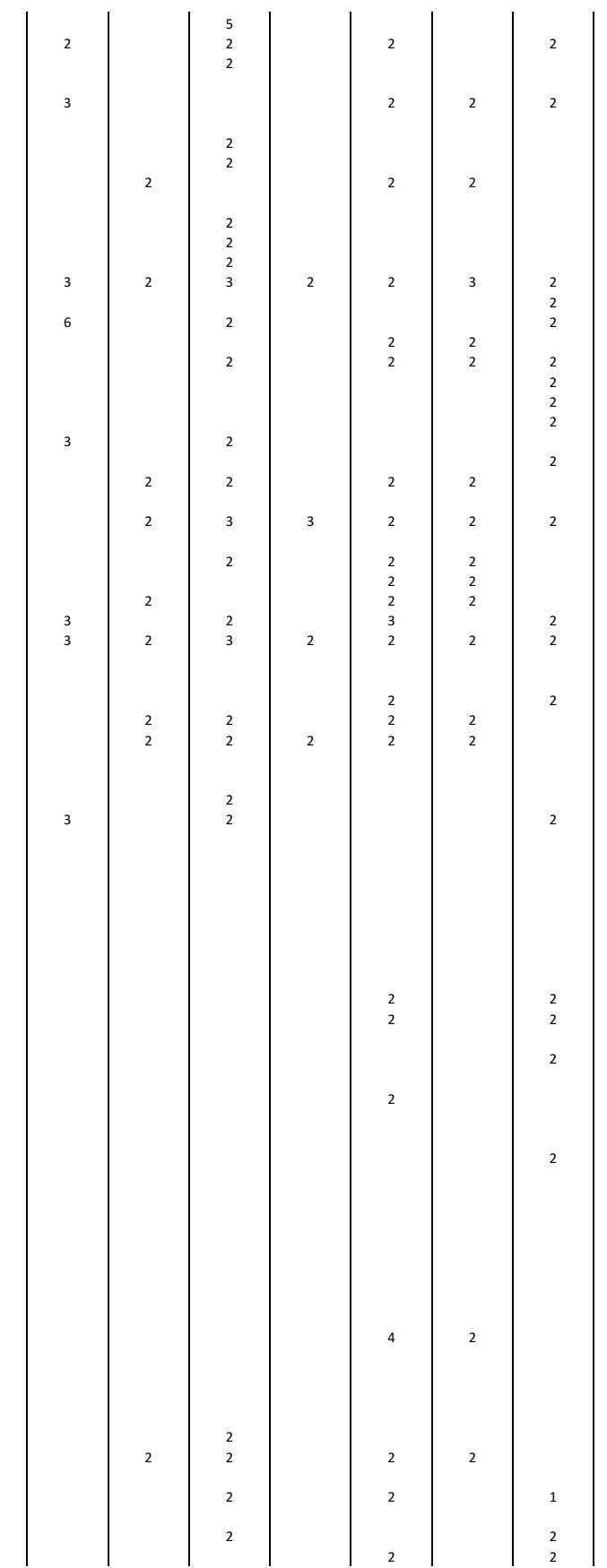




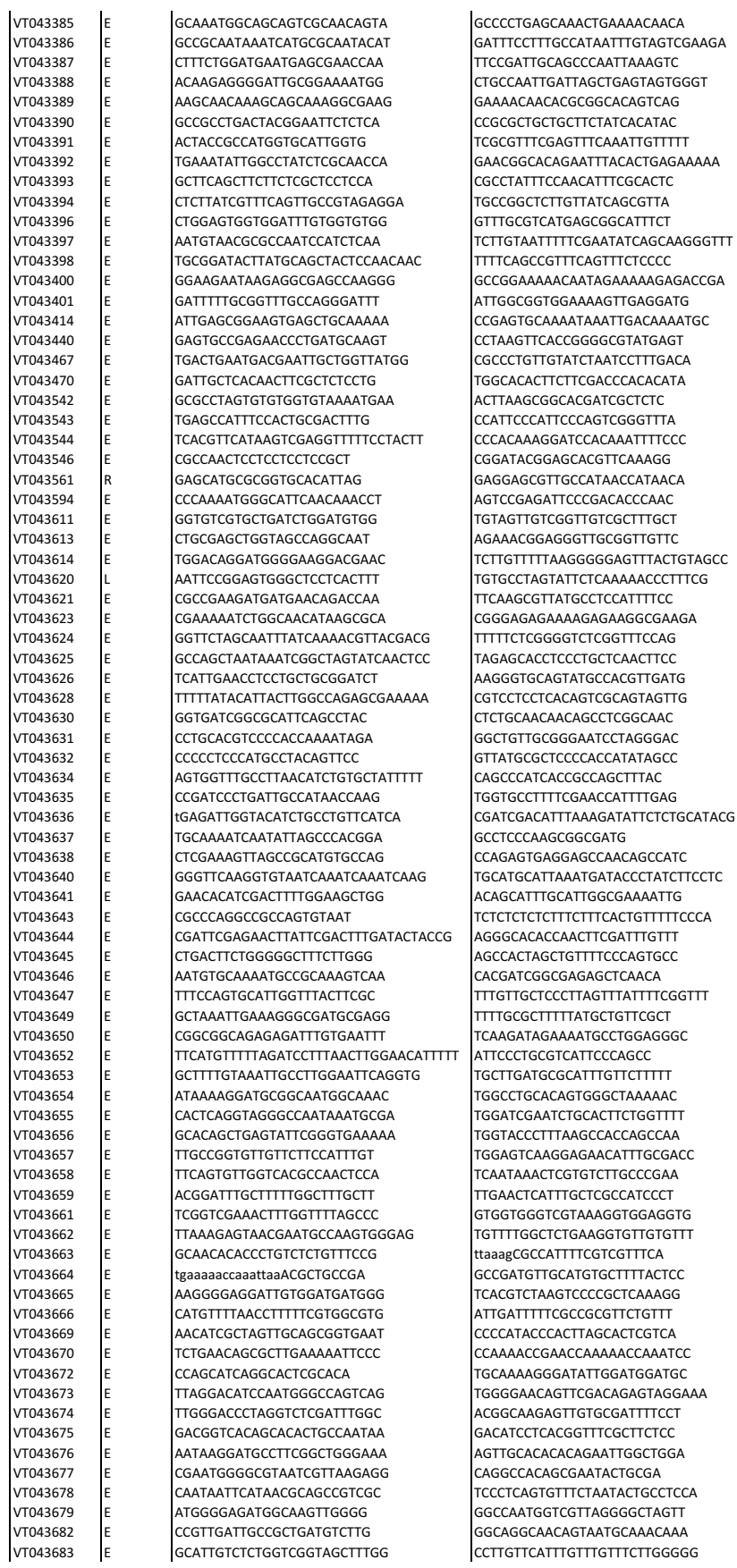

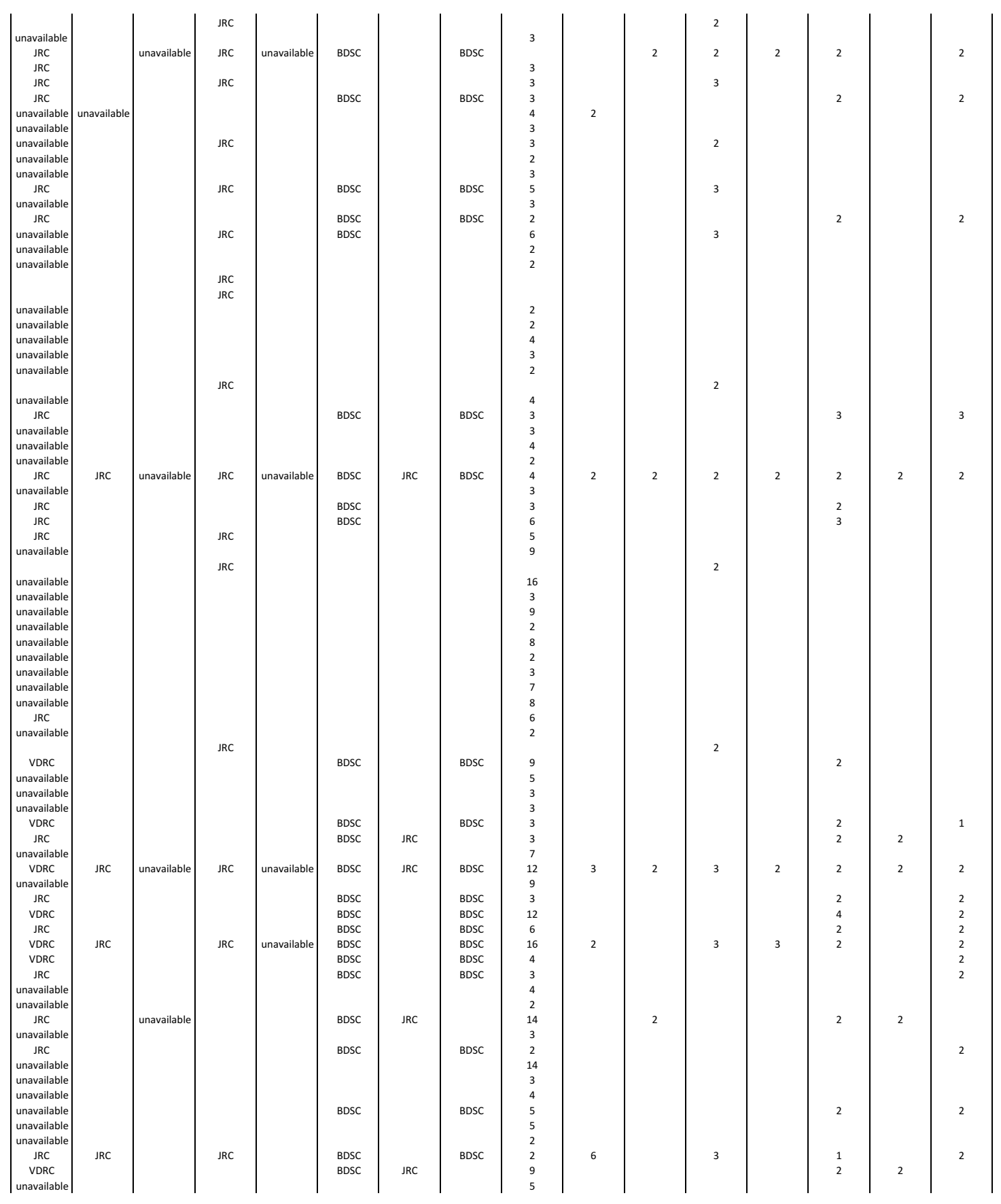




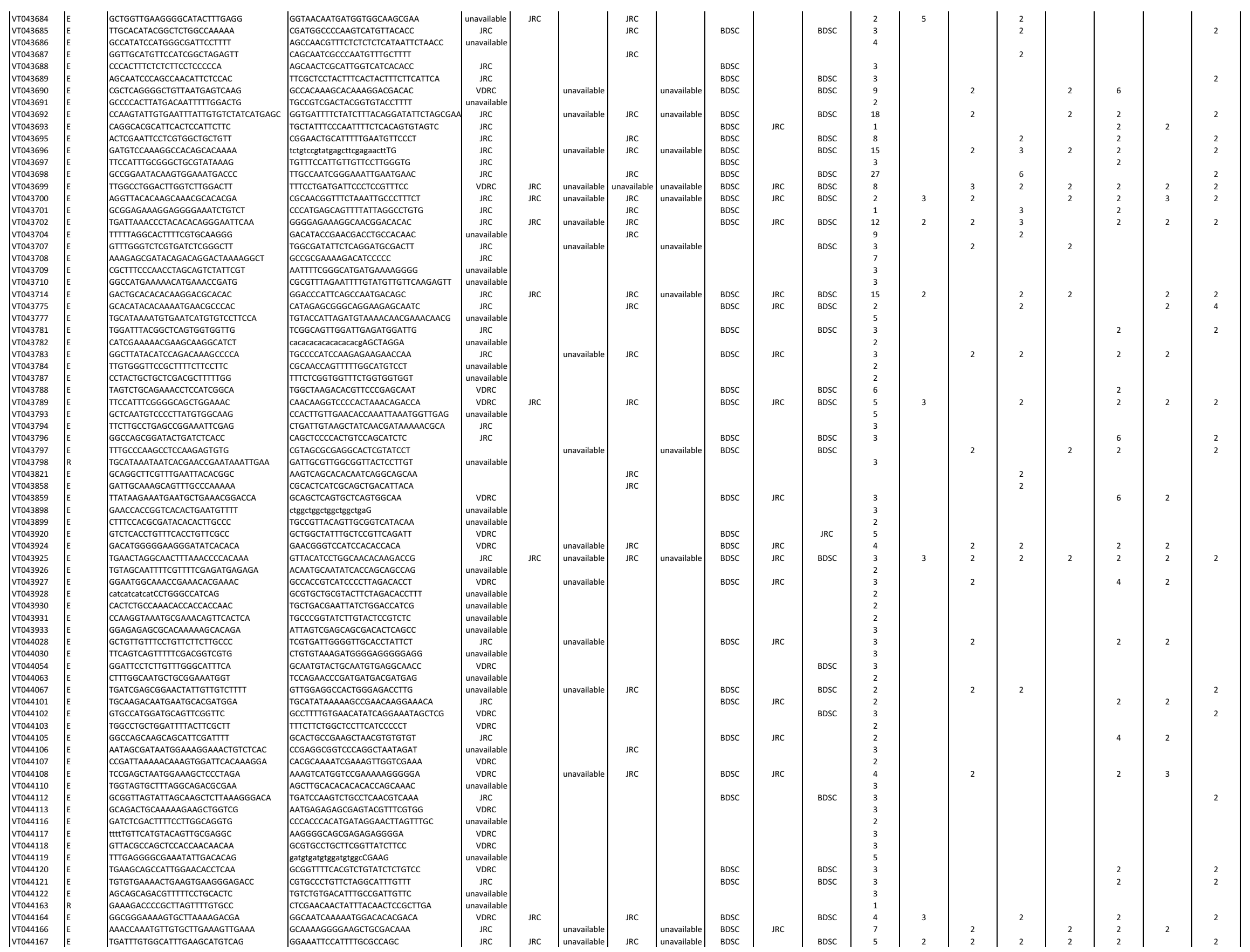




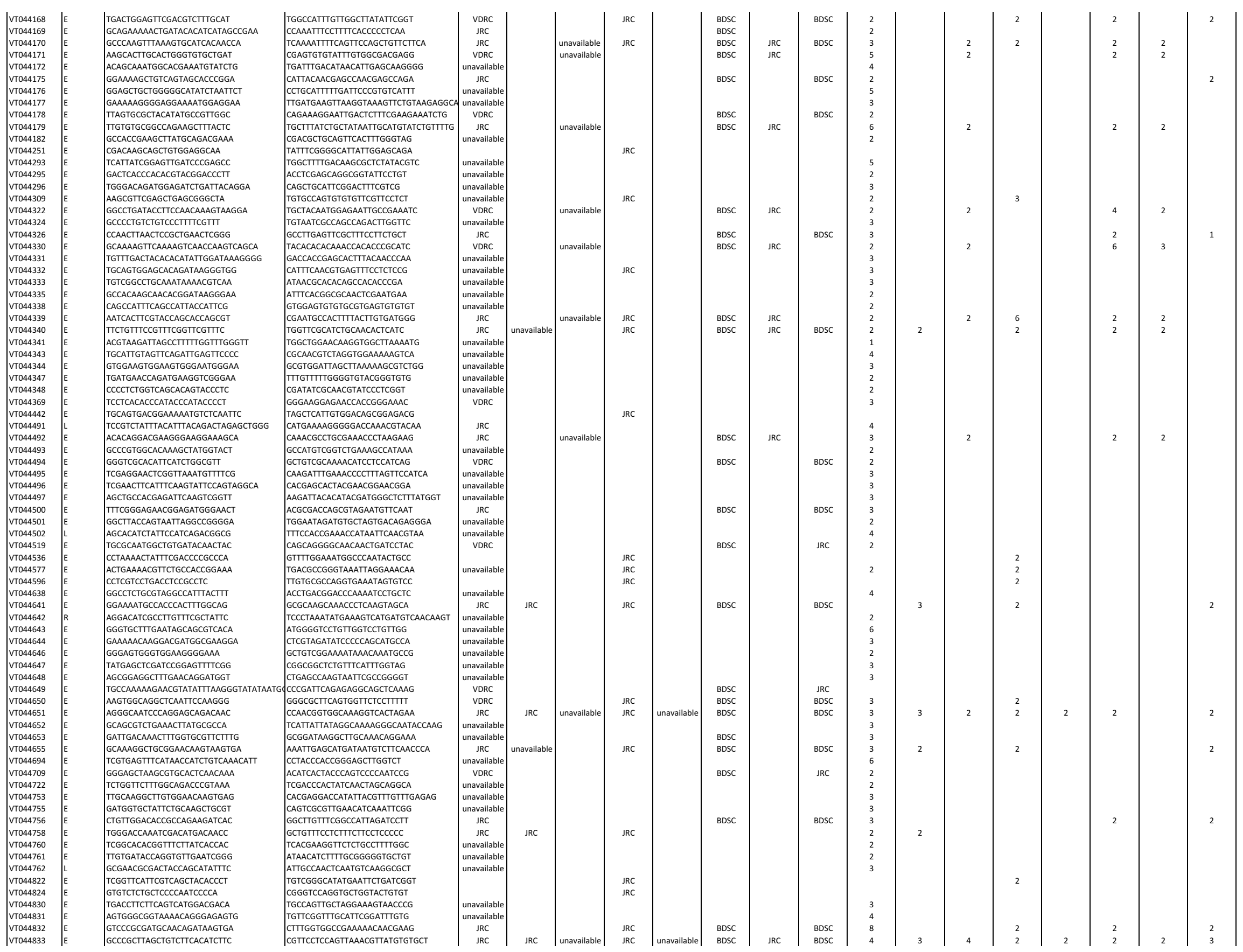




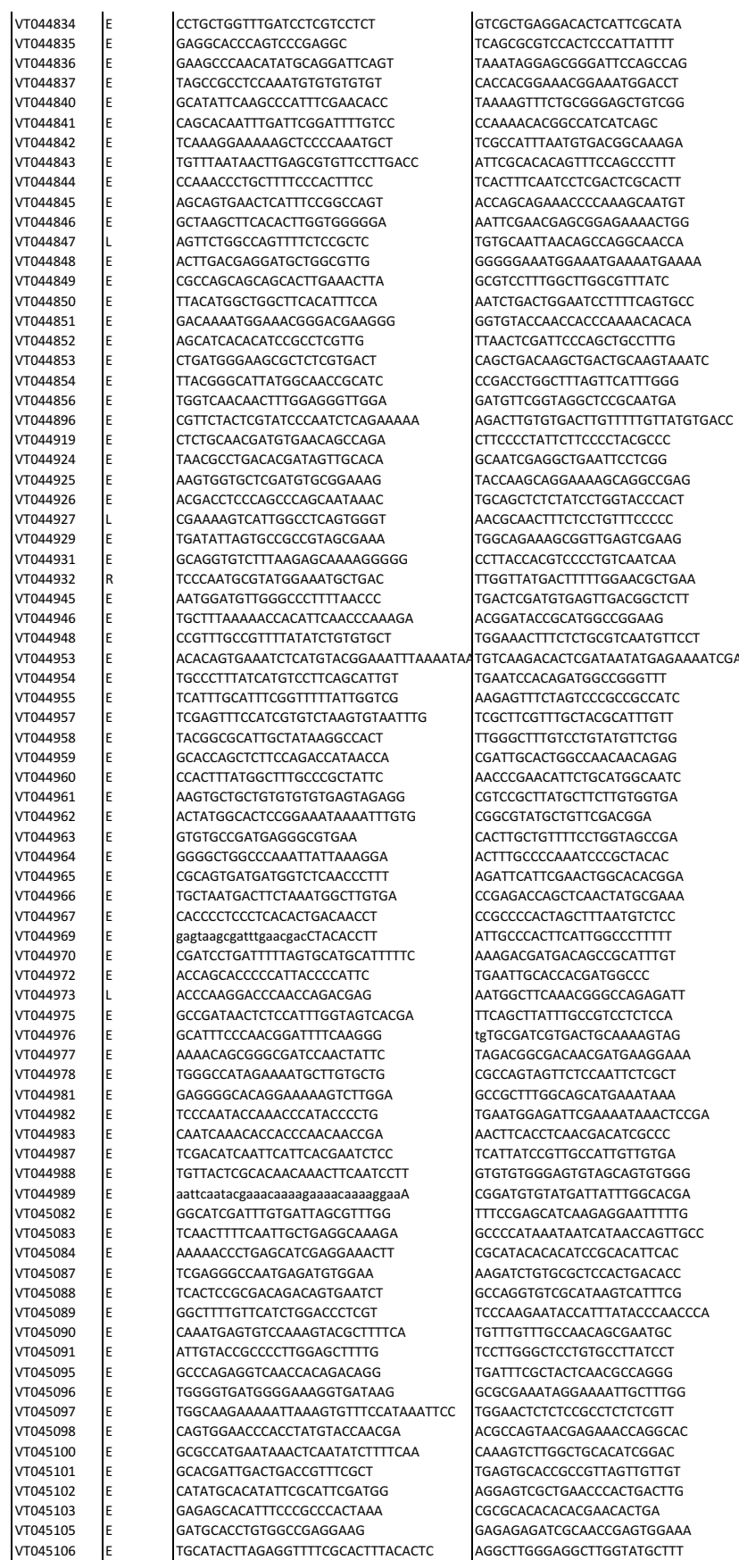
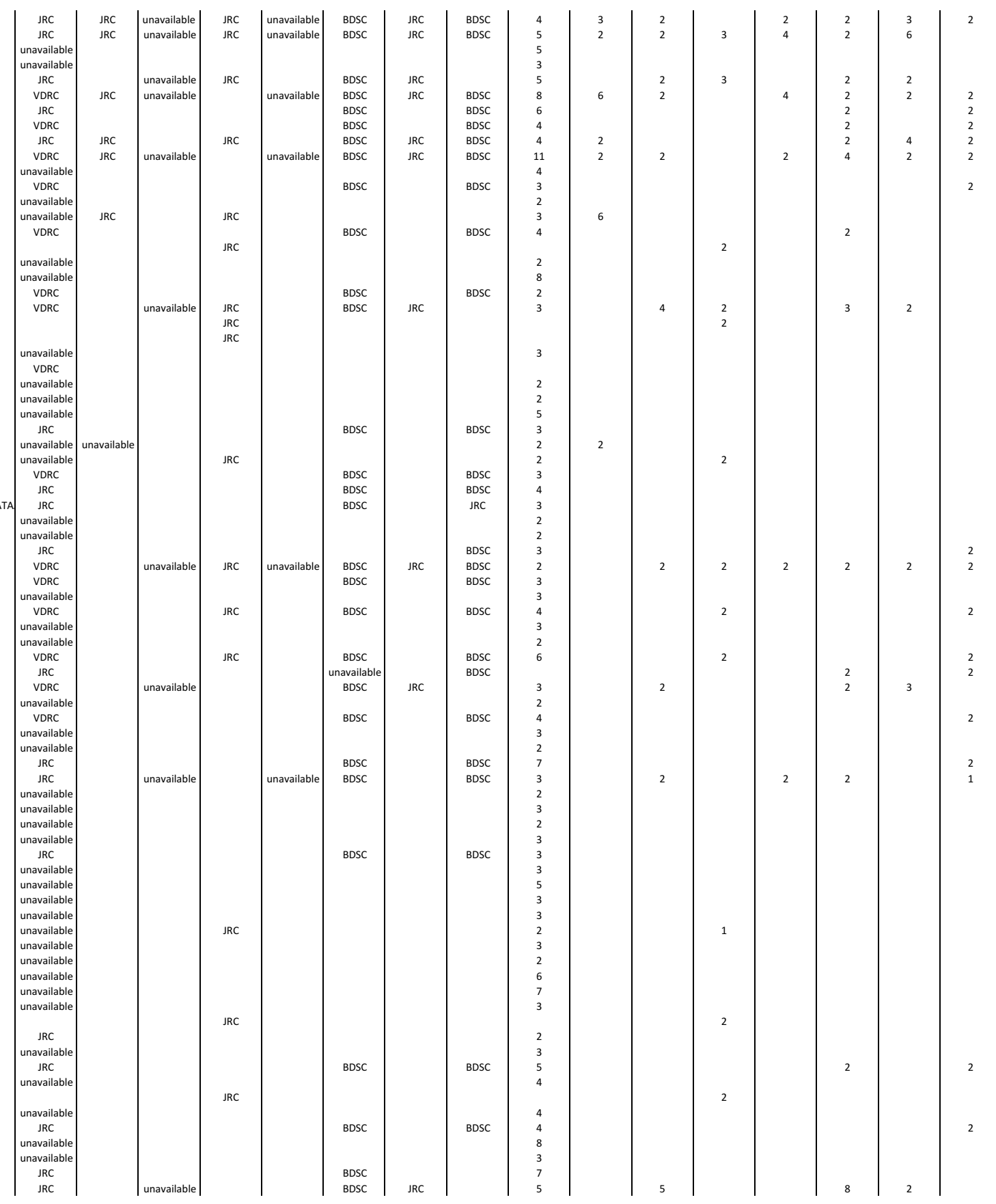


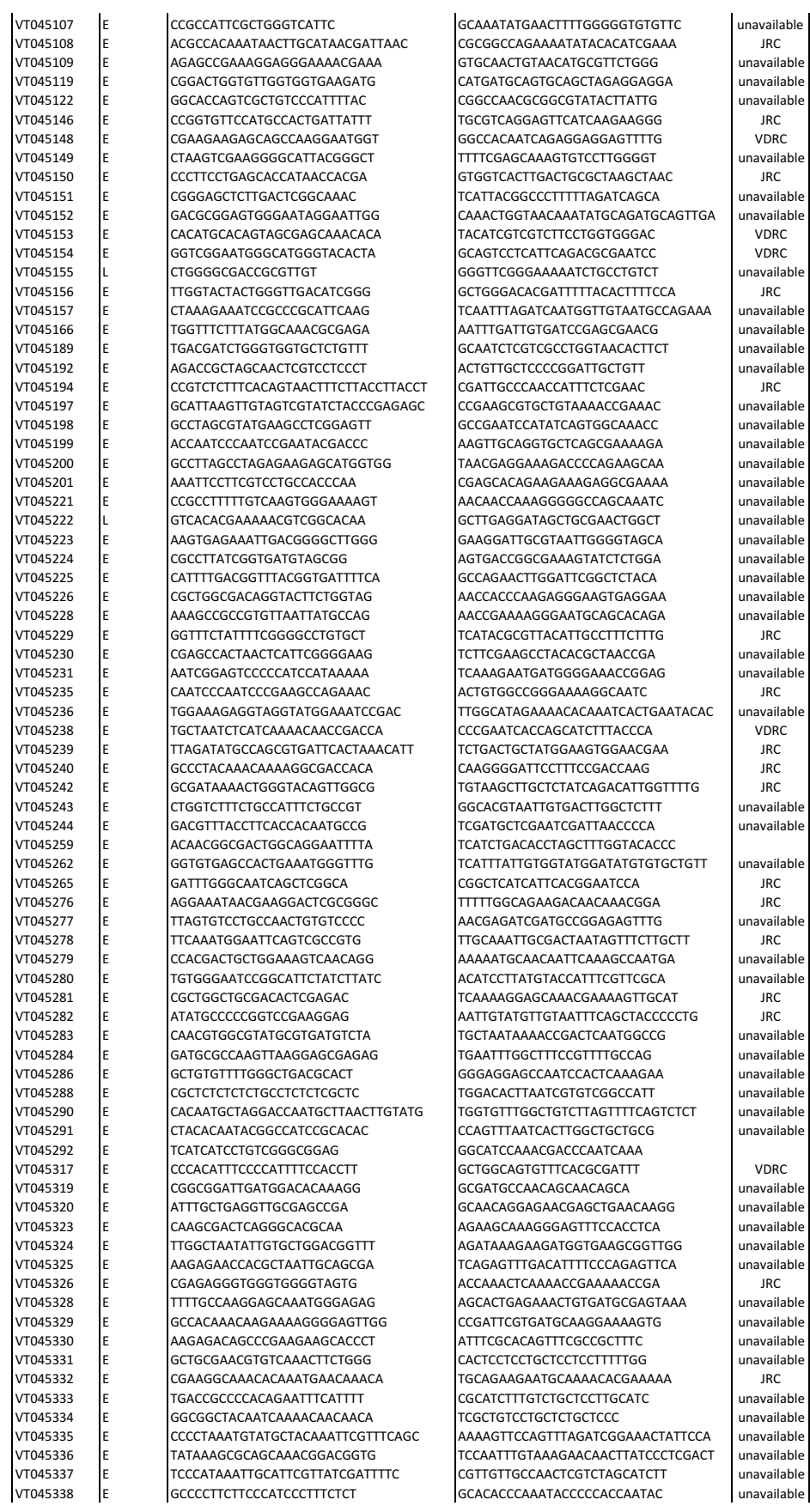
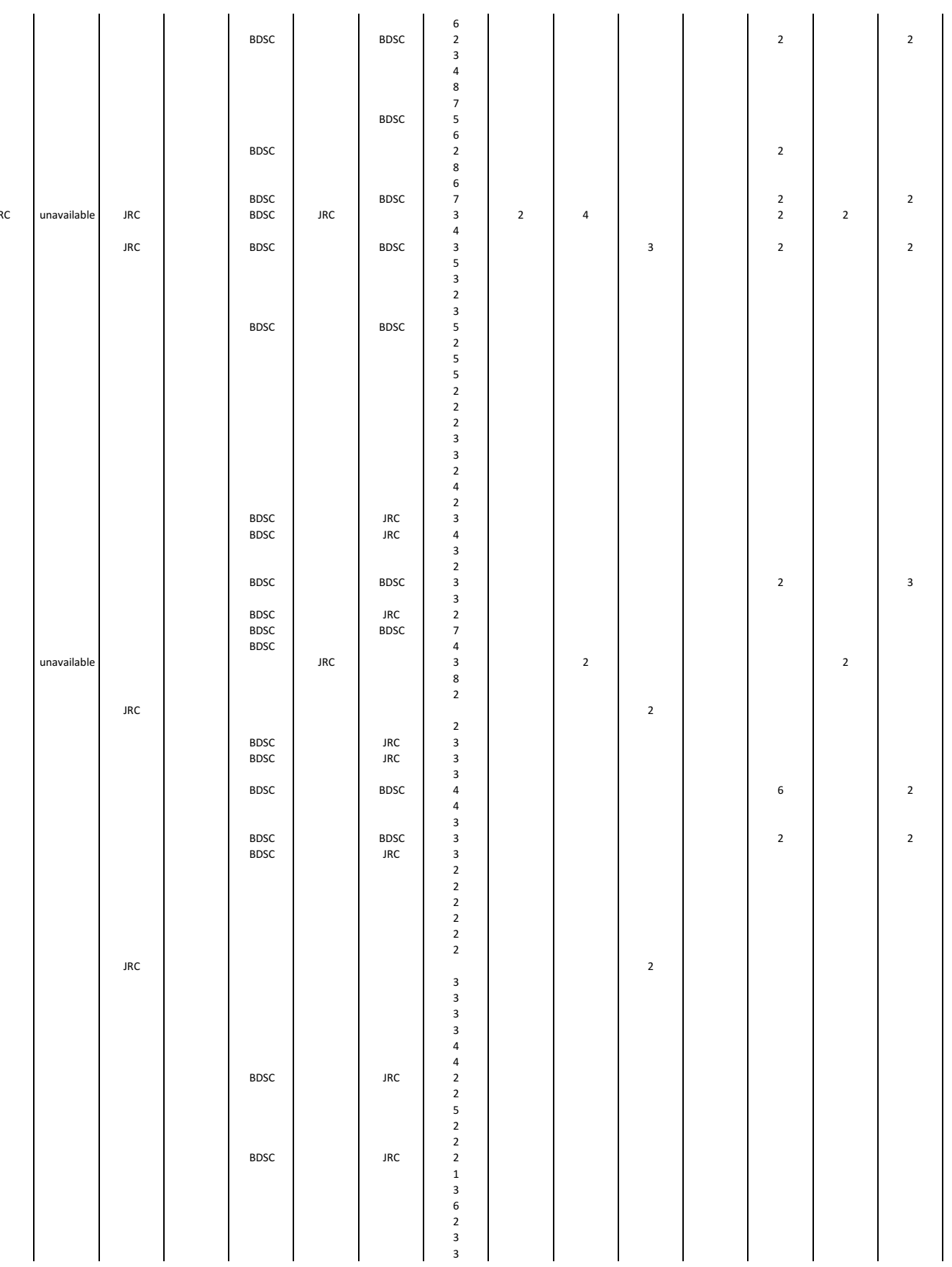


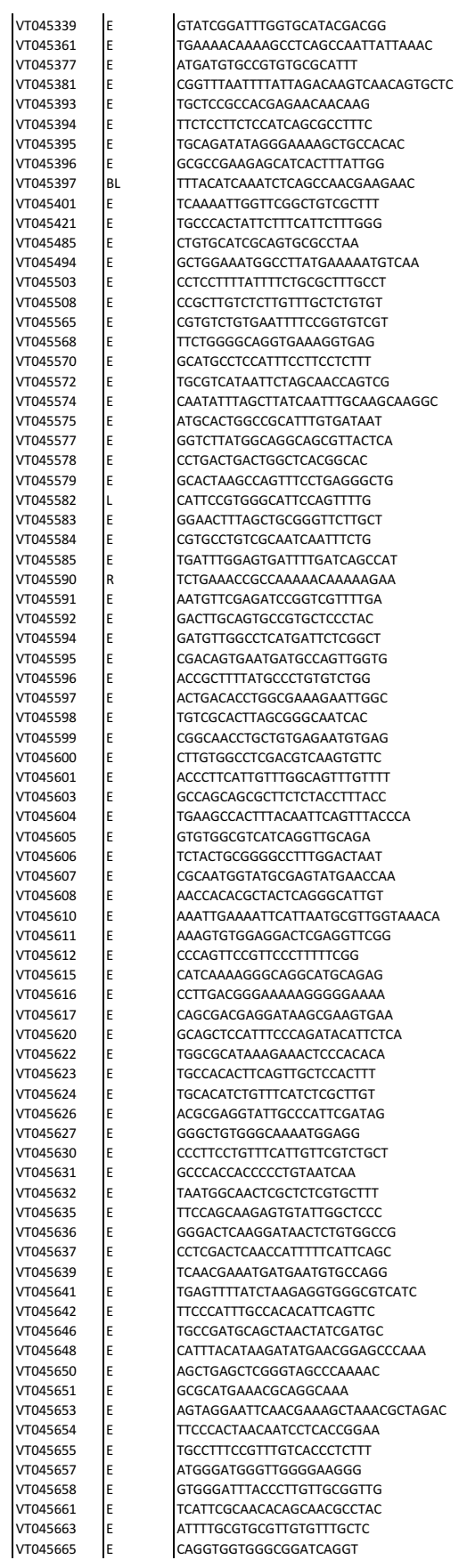

\begin{tabular}{|c|c|c|c|c|c|c|c|c|}
\hline 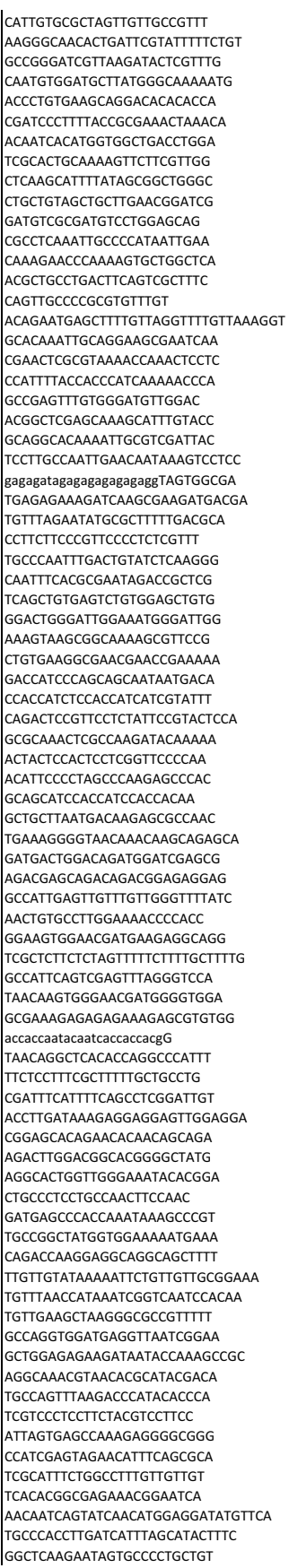 & 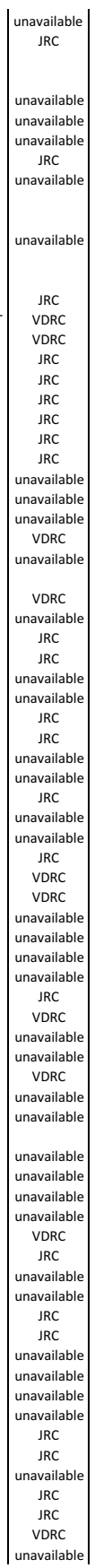 & \begin{tabular}{|c} 
JRC \\
unavailable
\end{tabular} & unavailable & $\begin{array}{l}\mathrm{JRC} \\
\mathrm{JRC} \\
\mathrm{JRC} \\
\mathrm{J} \mathrm{JC} \\
\mathrm{JRC} \\
\mathrm{JRC}\end{array}$ & JRC & $\begin{array}{l}\text { BDSC } \\
\text { BDSC } \\
\text { BDSC } \\
\text { BDSC } \\
\text { BDSC } \\
\text { BDSC } \\
\text { BDSC }\end{array}$ & $\begin{array}{l}\text { JRC } \\
\text { JRC }\end{array}$ & $\begin{array}{l}\text { BDSC } \\
\text { BDSC } \\
\text { BDSC } \\
\text { BDSC }\end{array}$ \\
\hline
\end{tabular}
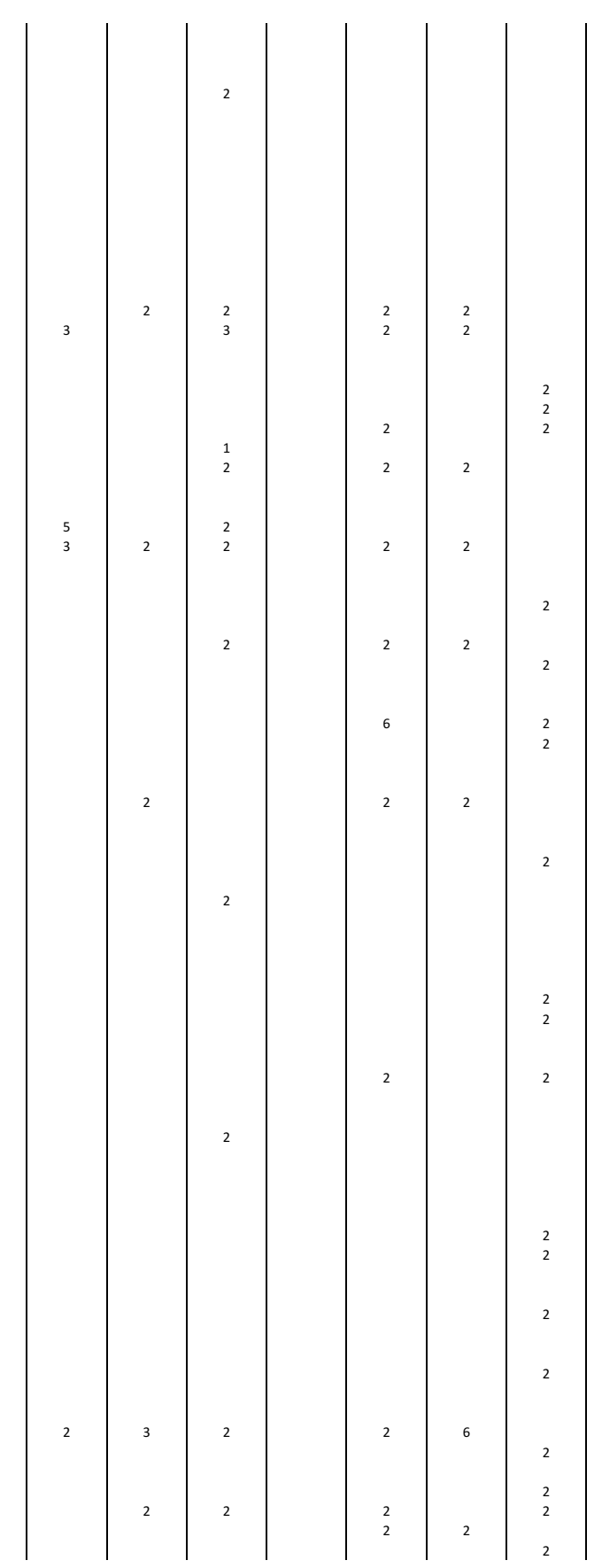


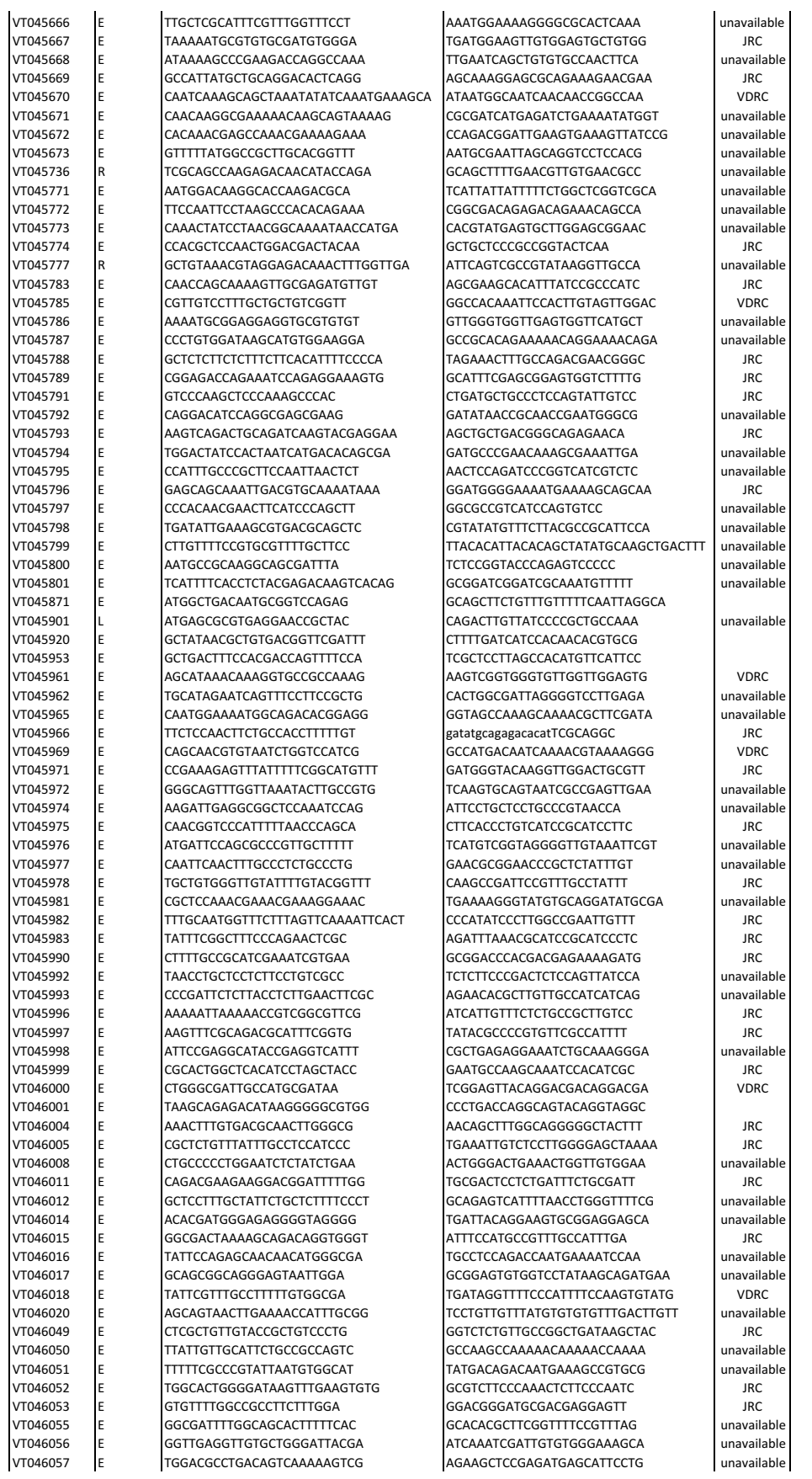
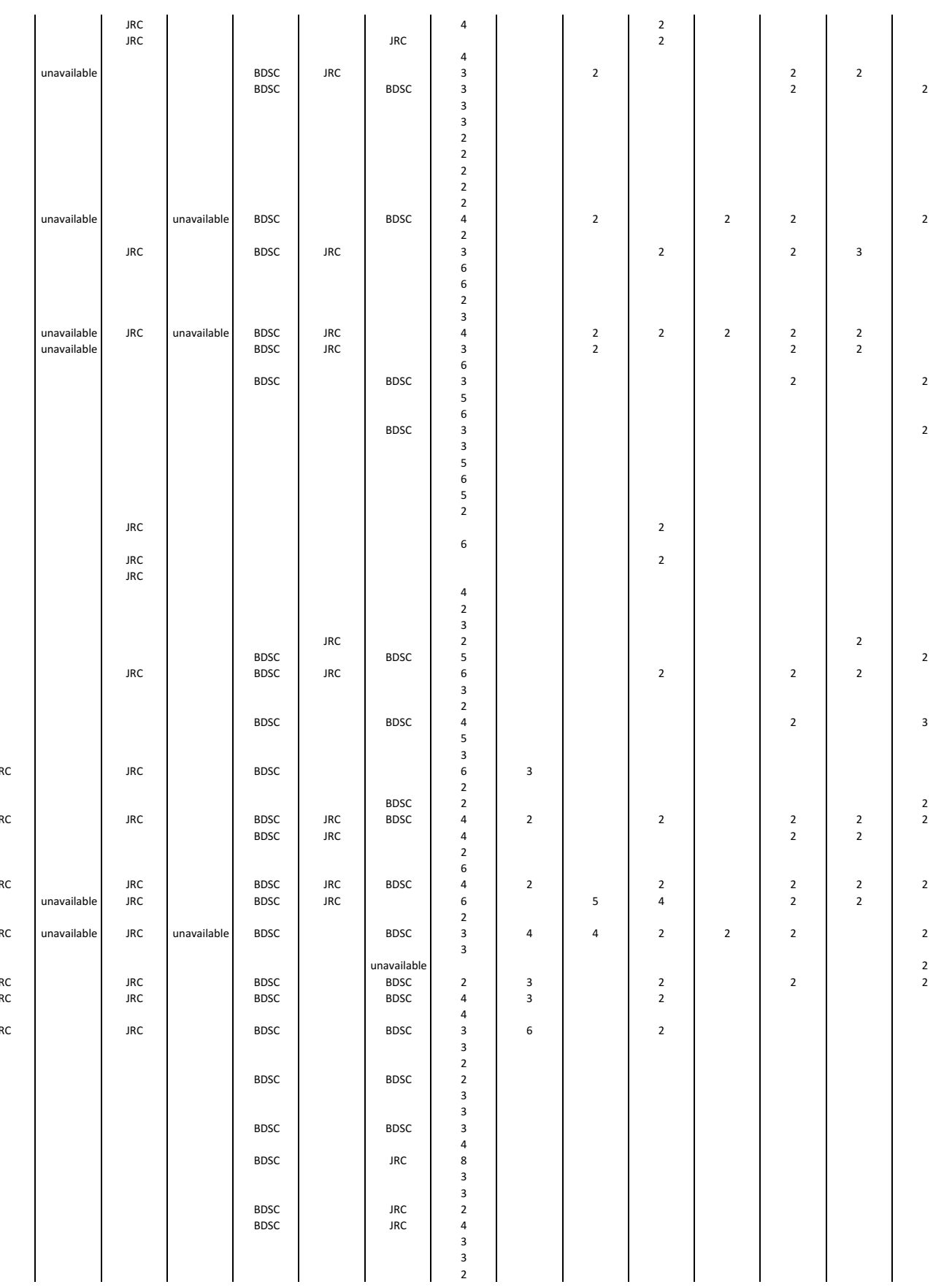


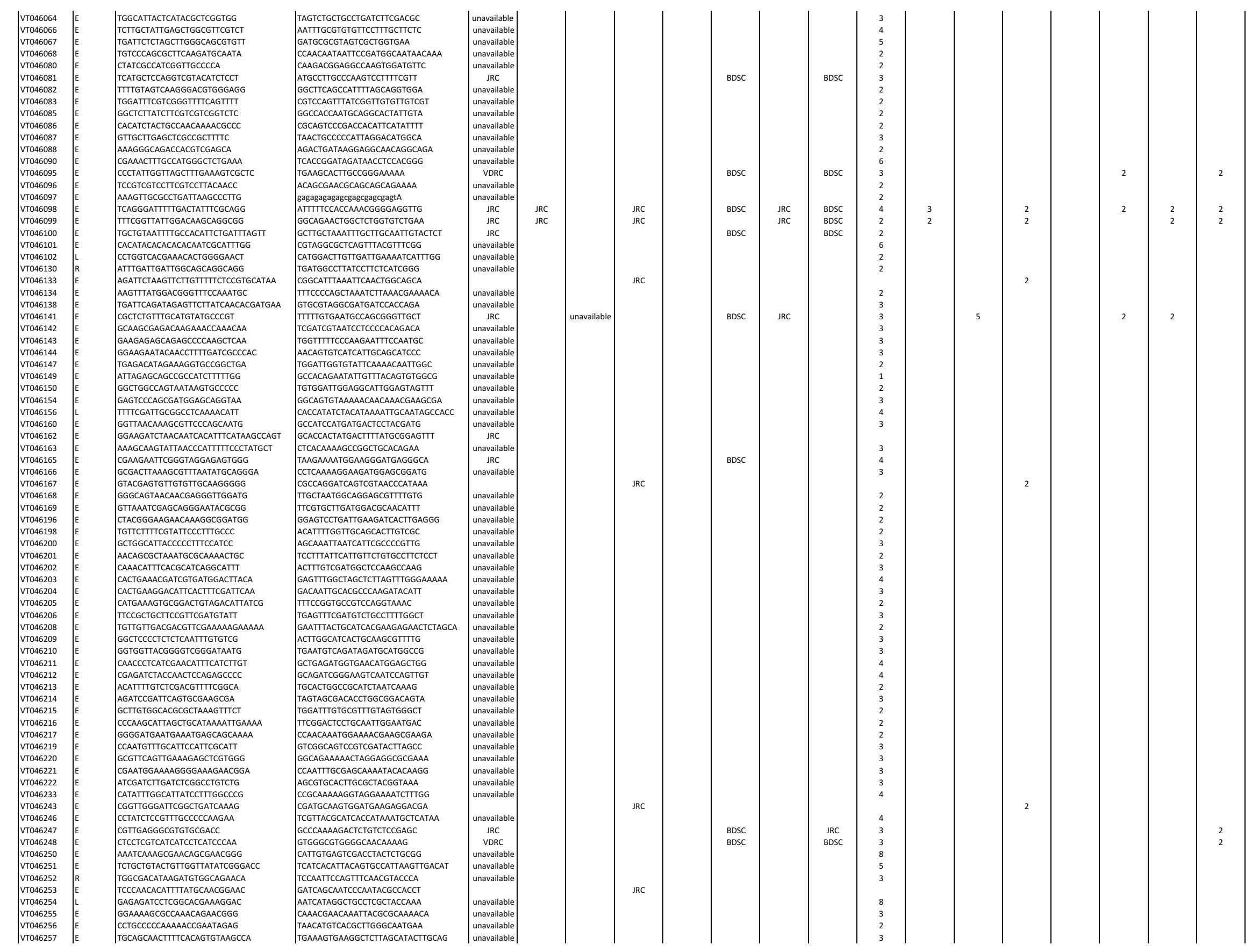




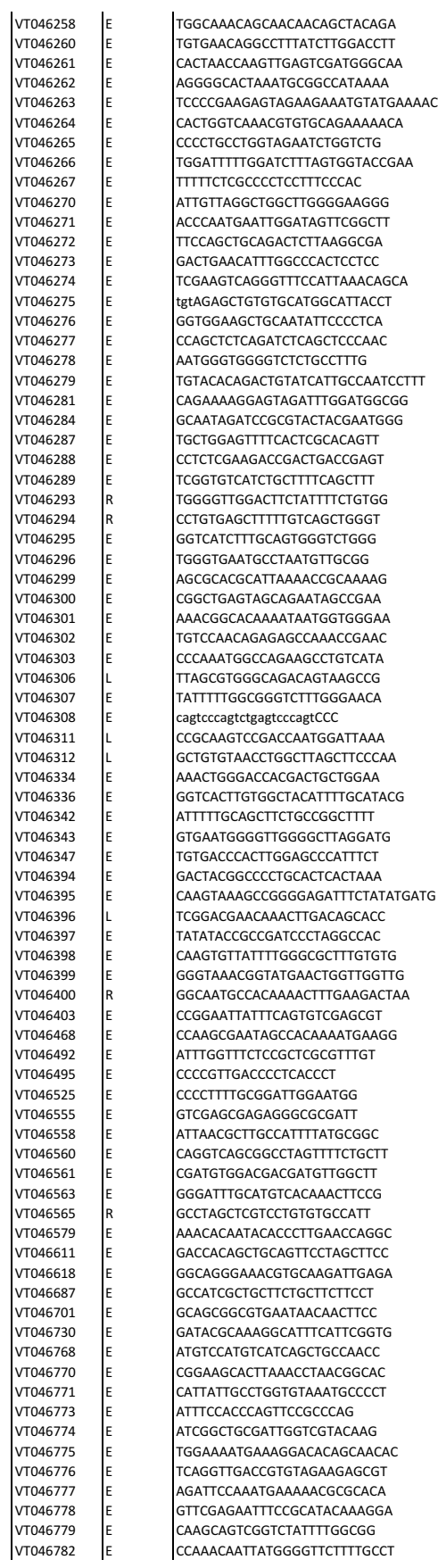
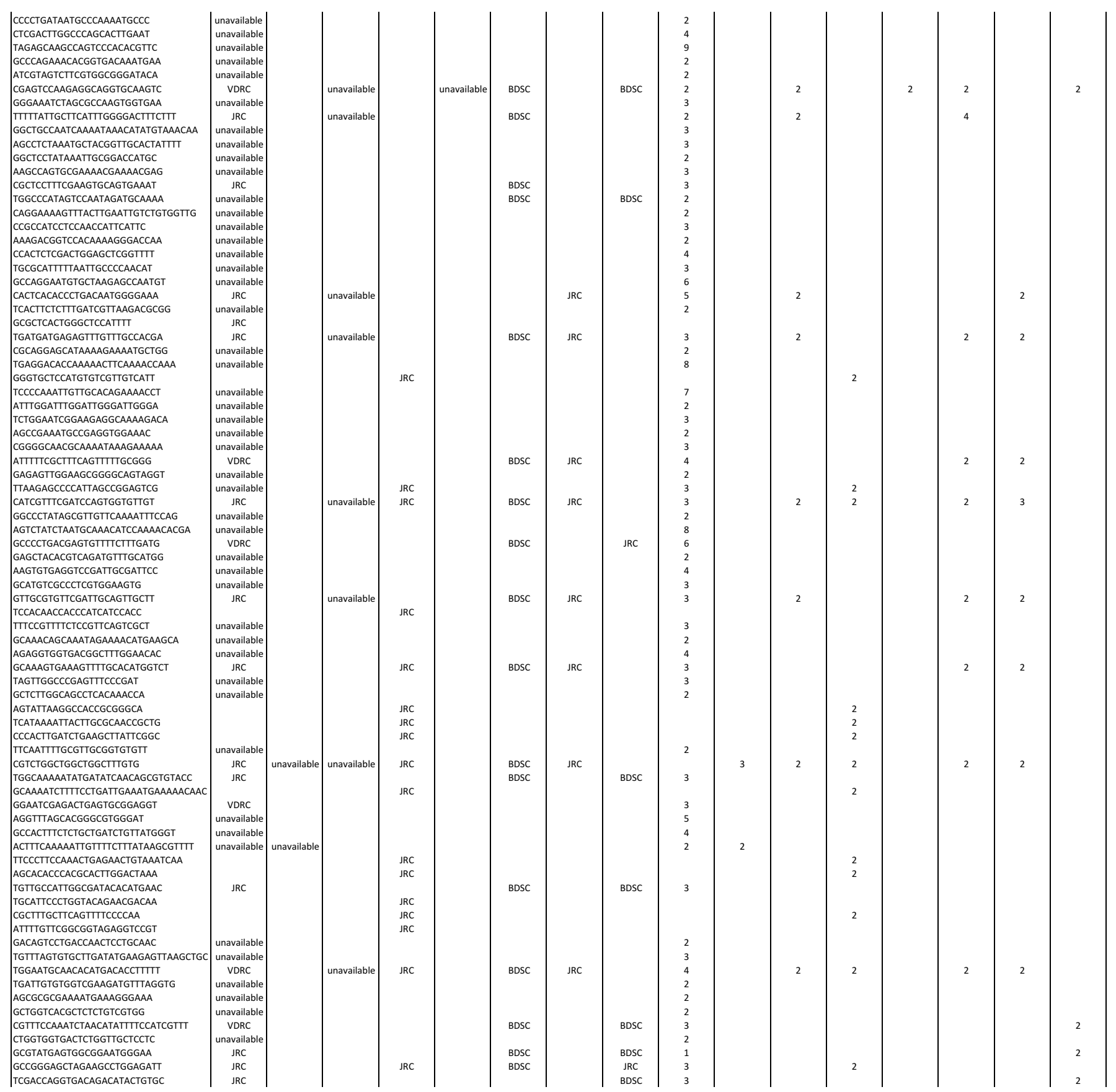


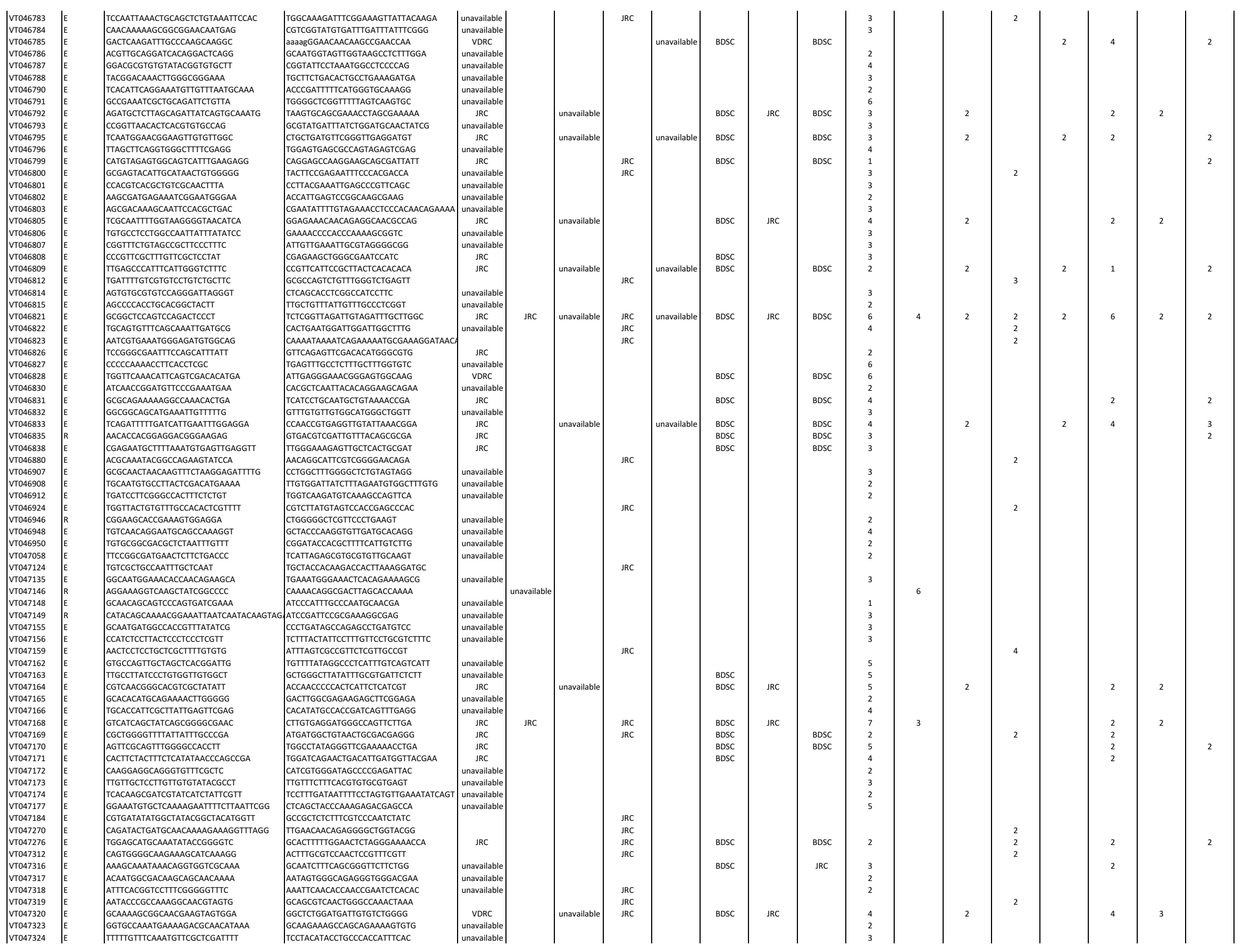




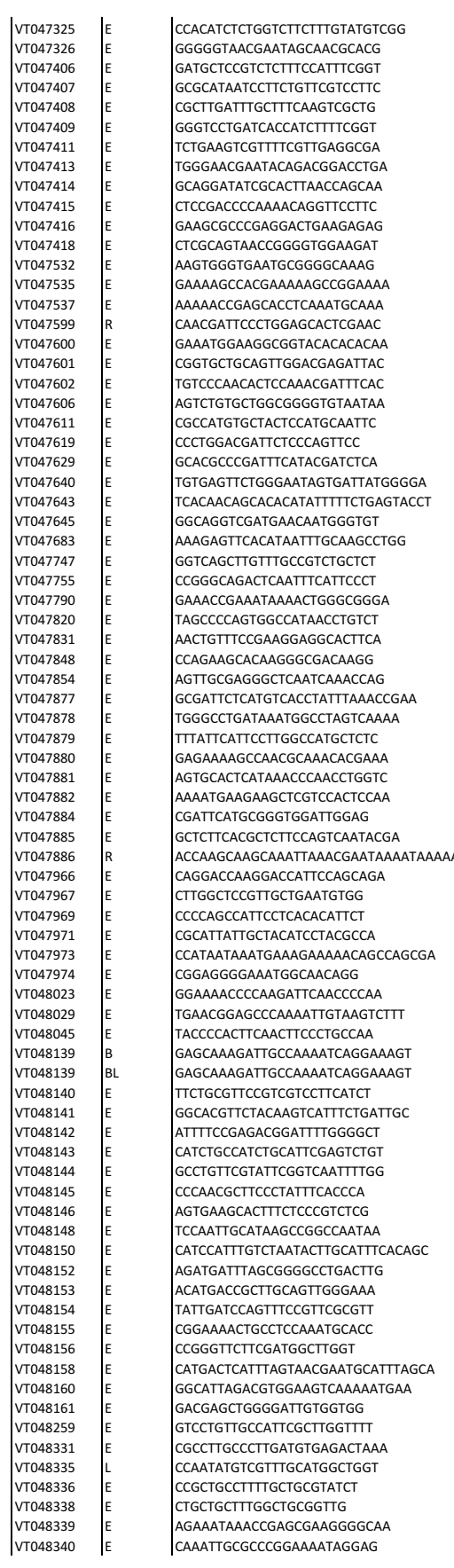

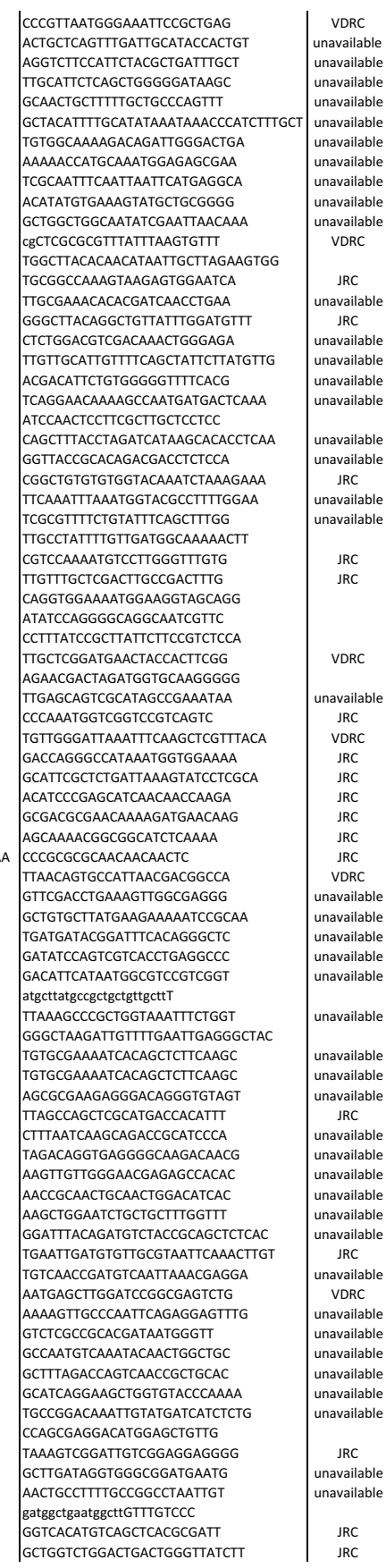
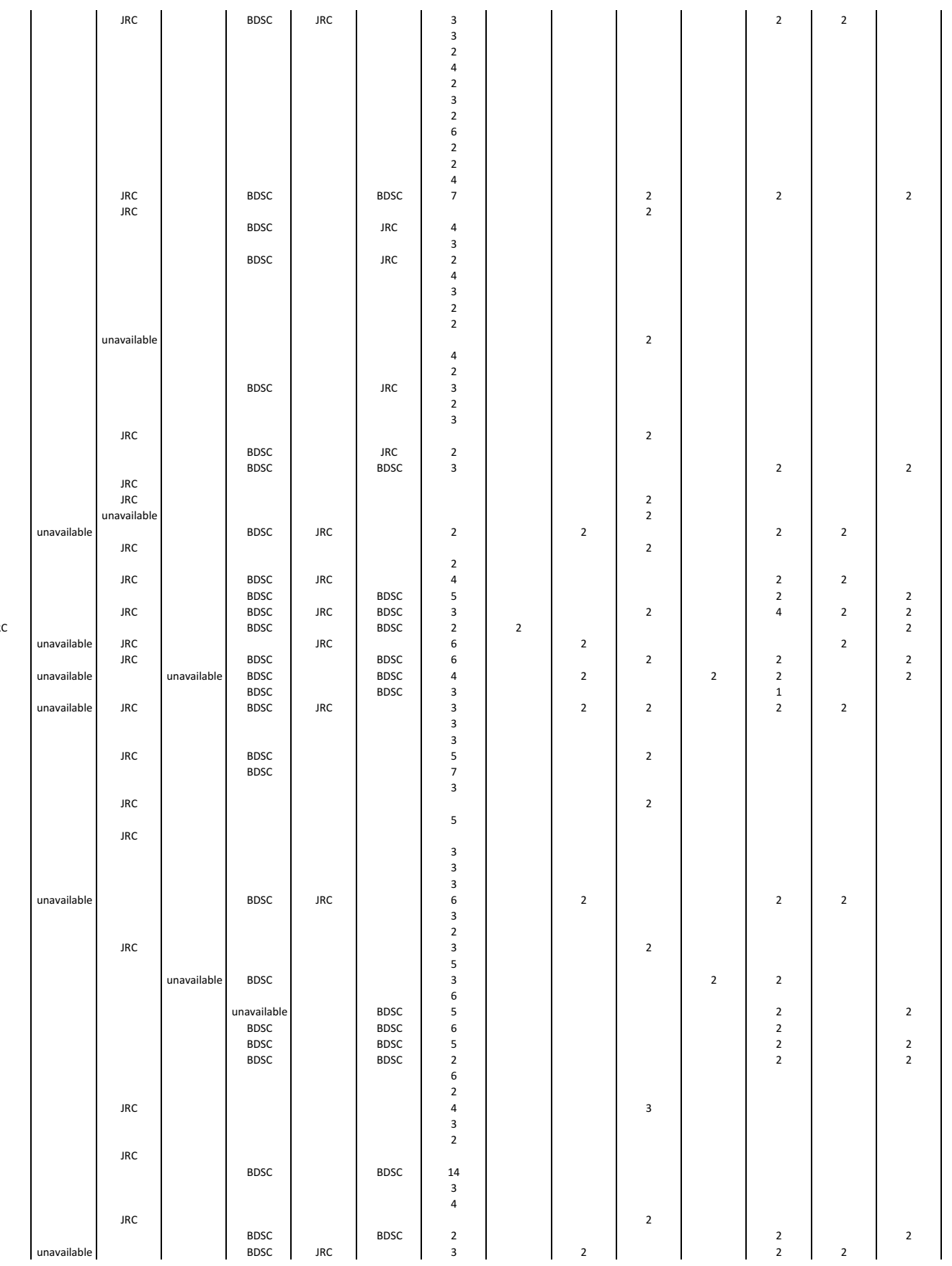


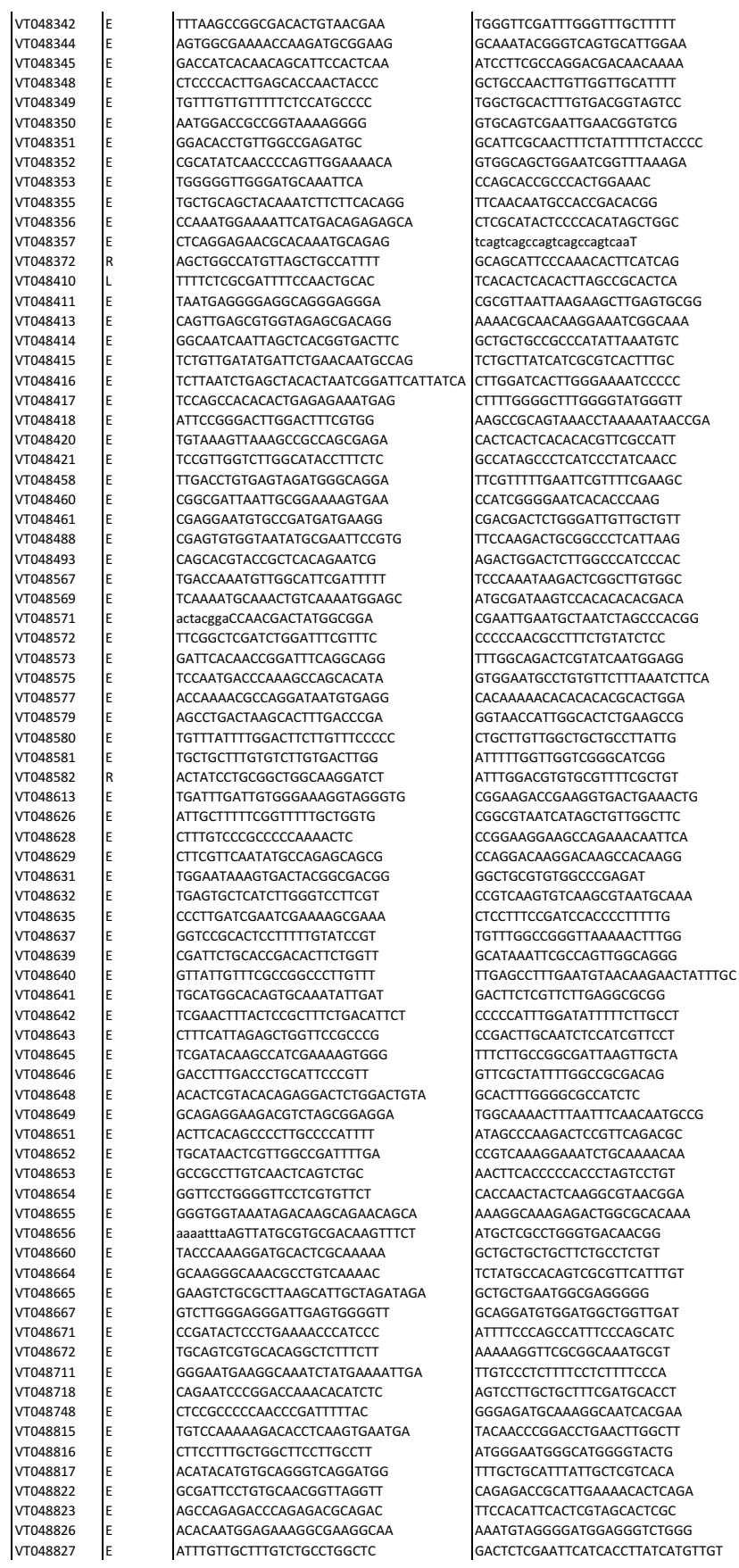

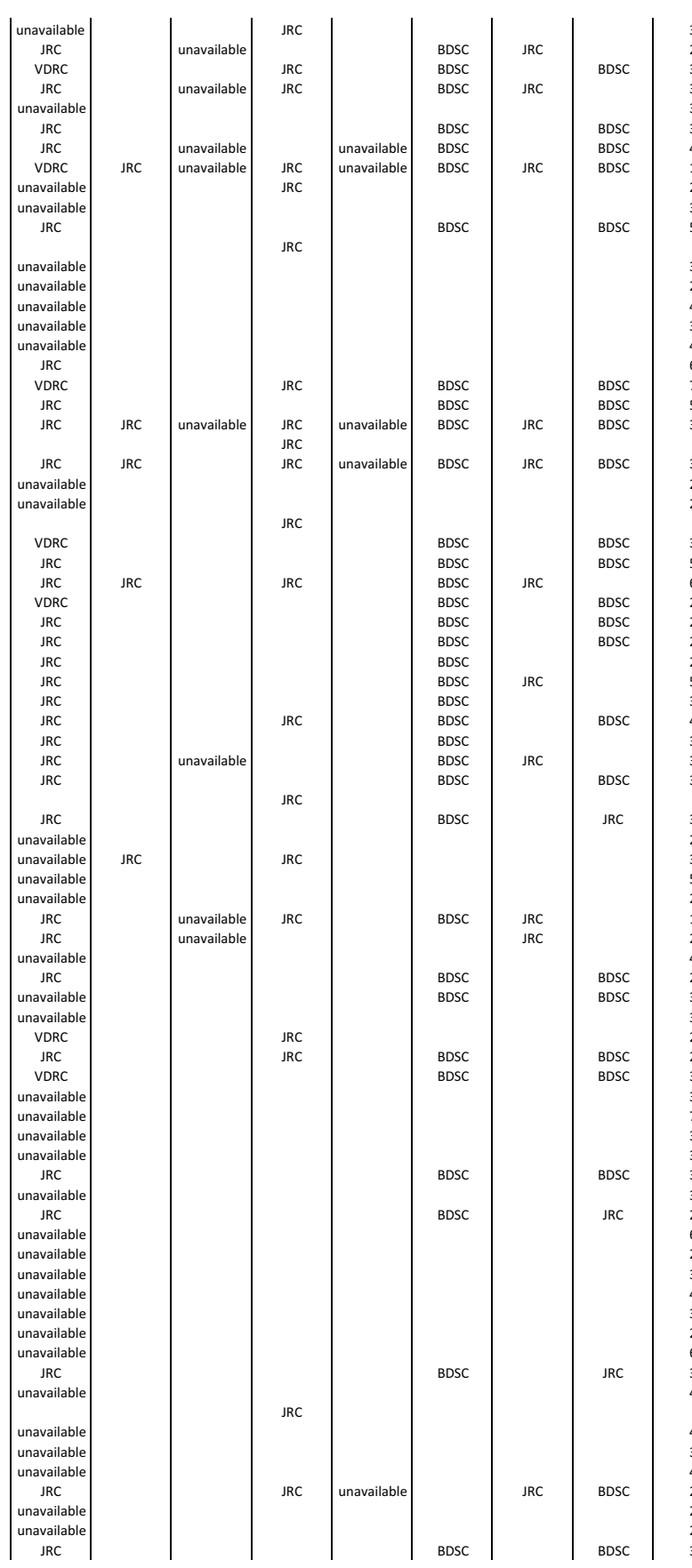




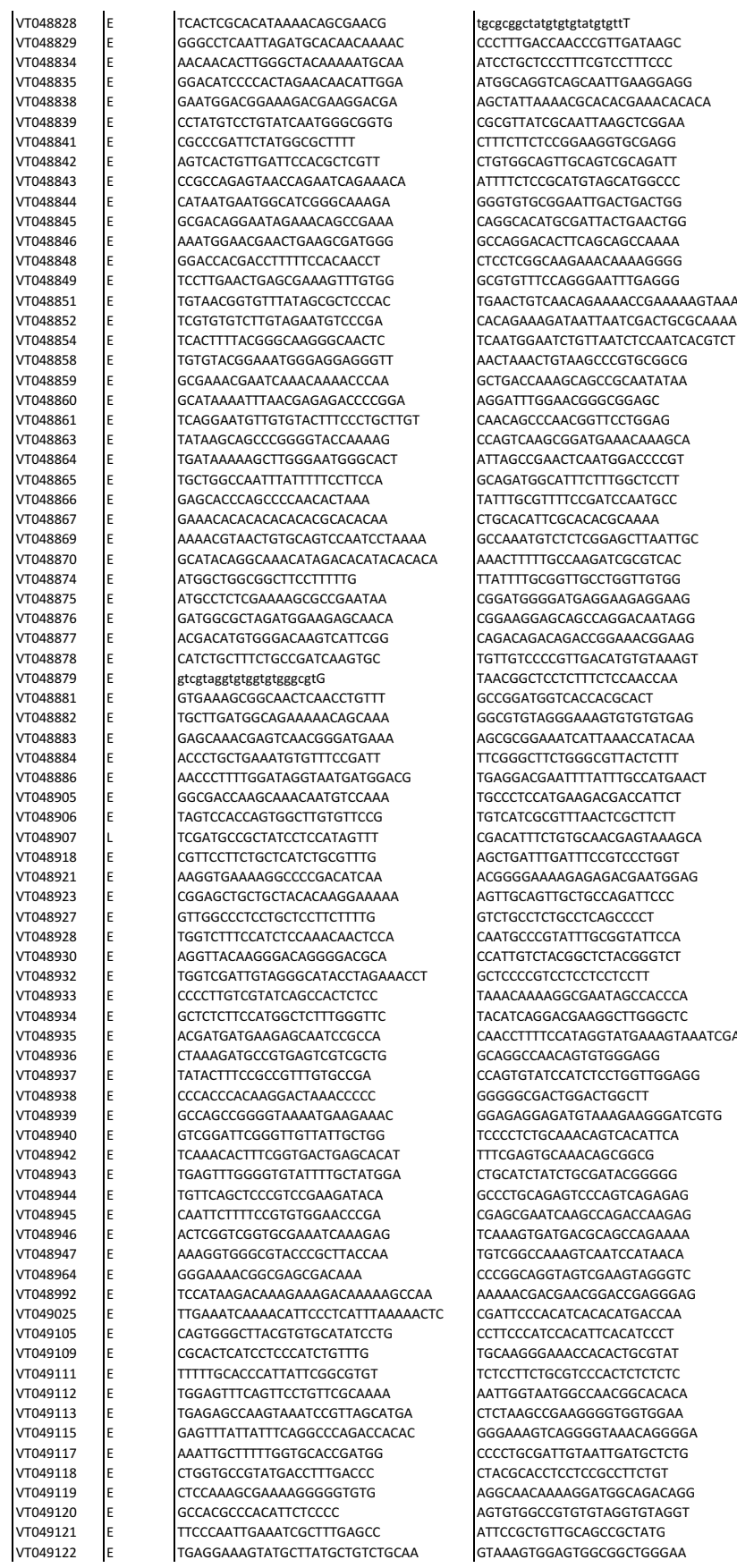
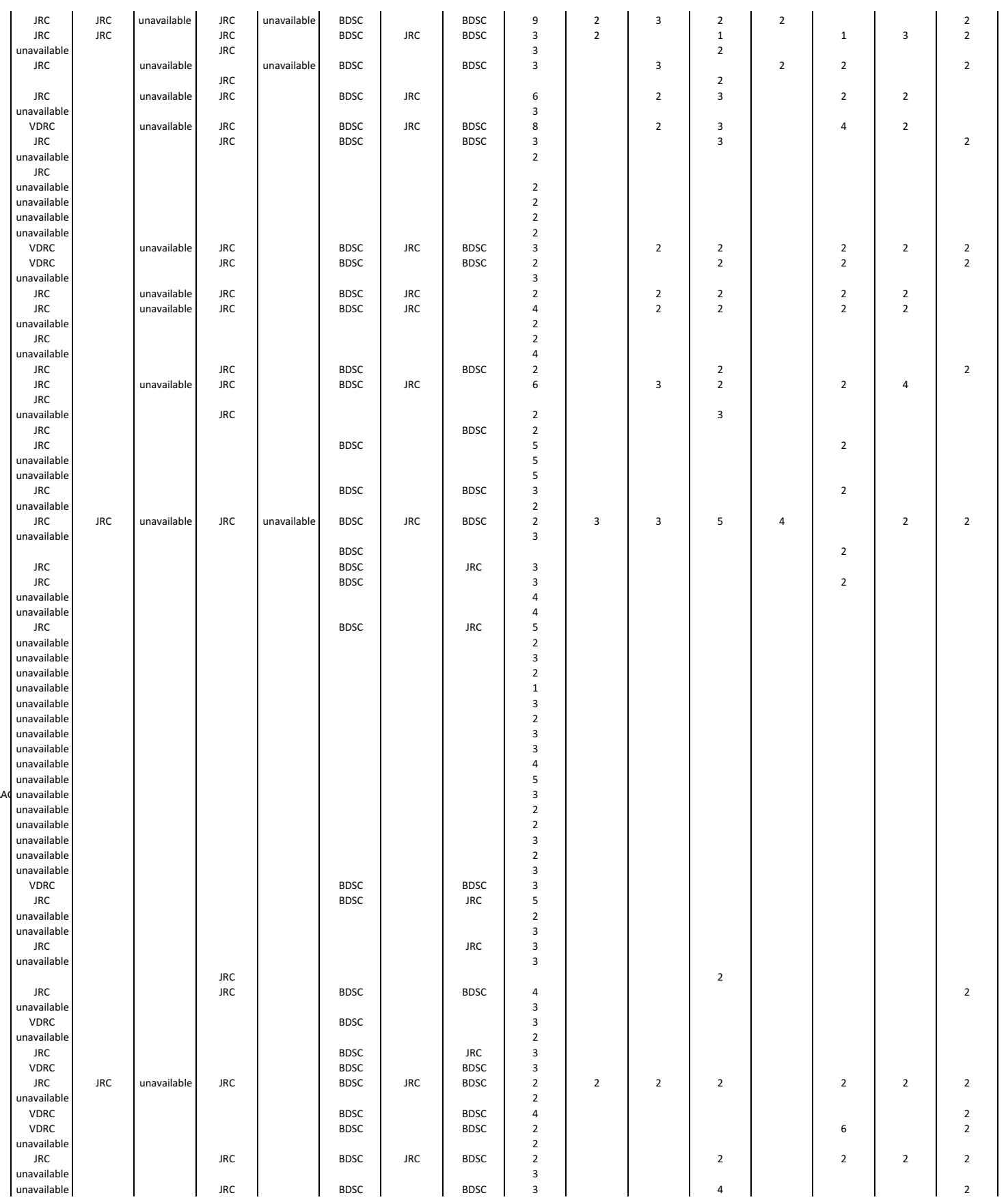


\begin{tabular}{|c|c|c|}
\hline \multicolumn{2}{|c|}{ 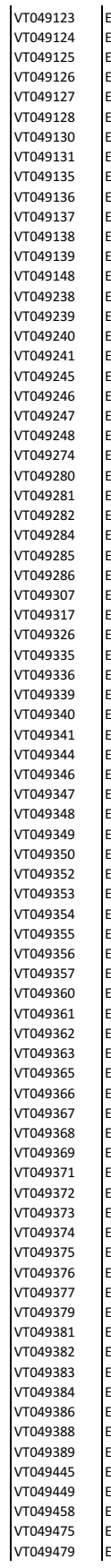 } & 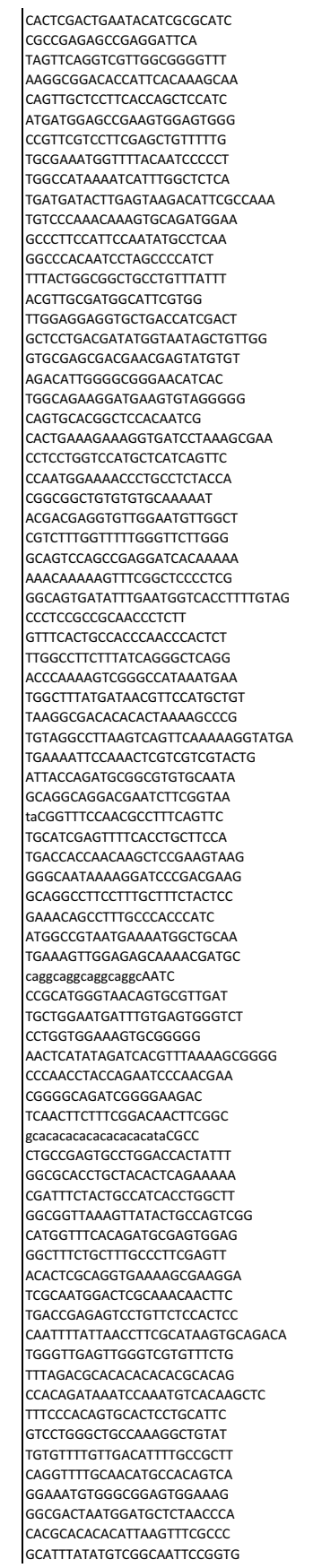 \\
\hline
\end{tabular}

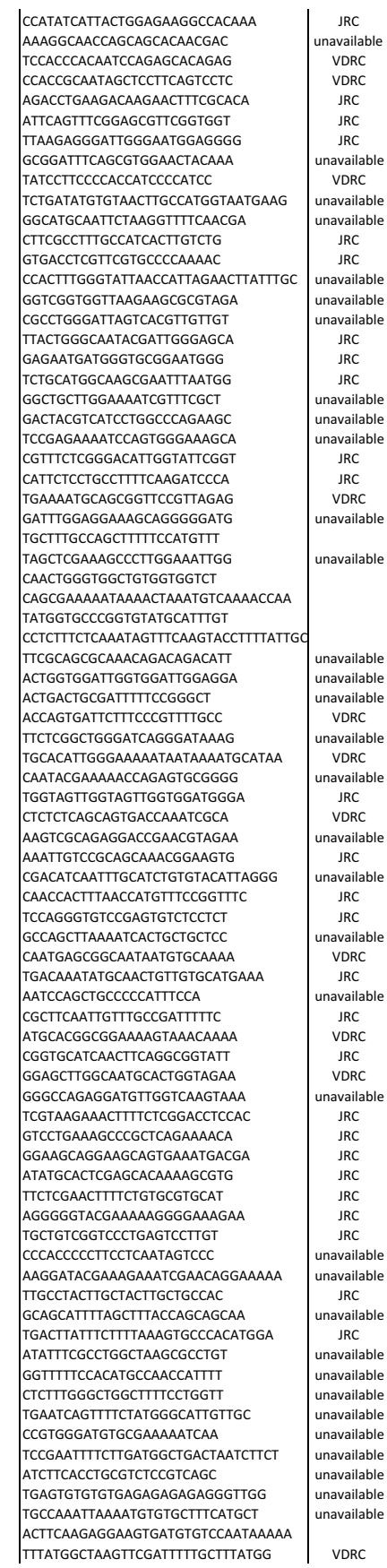
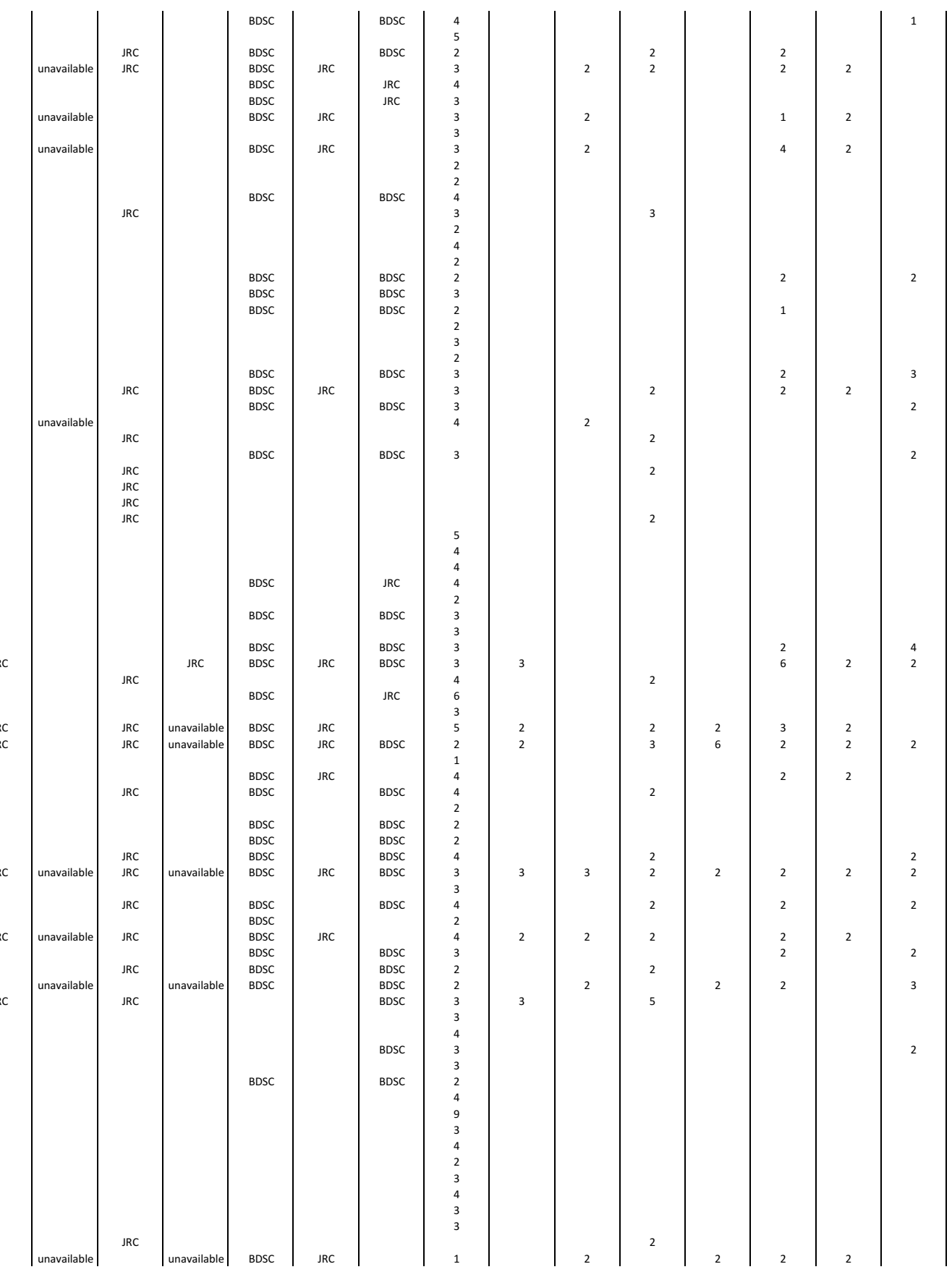


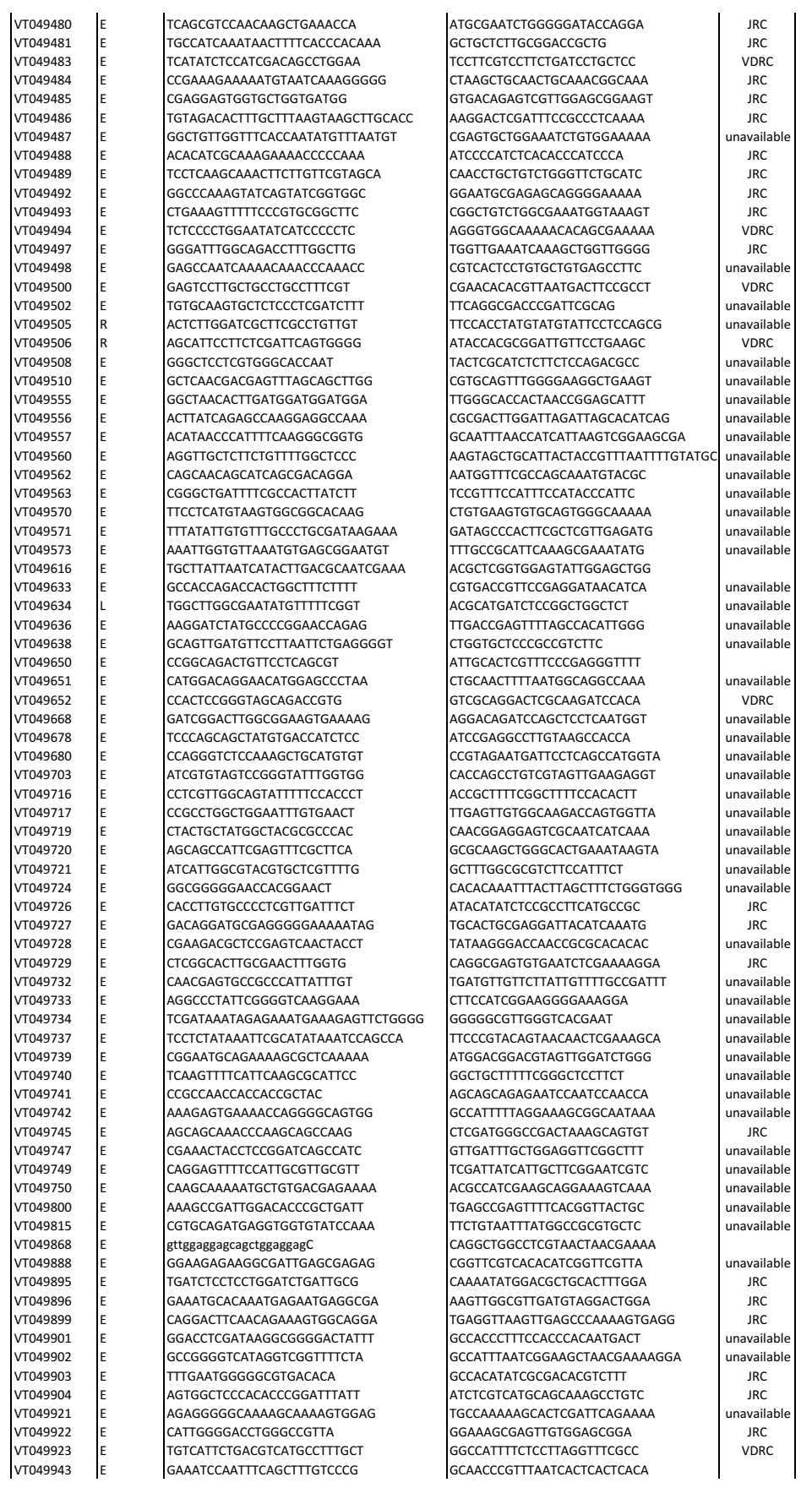
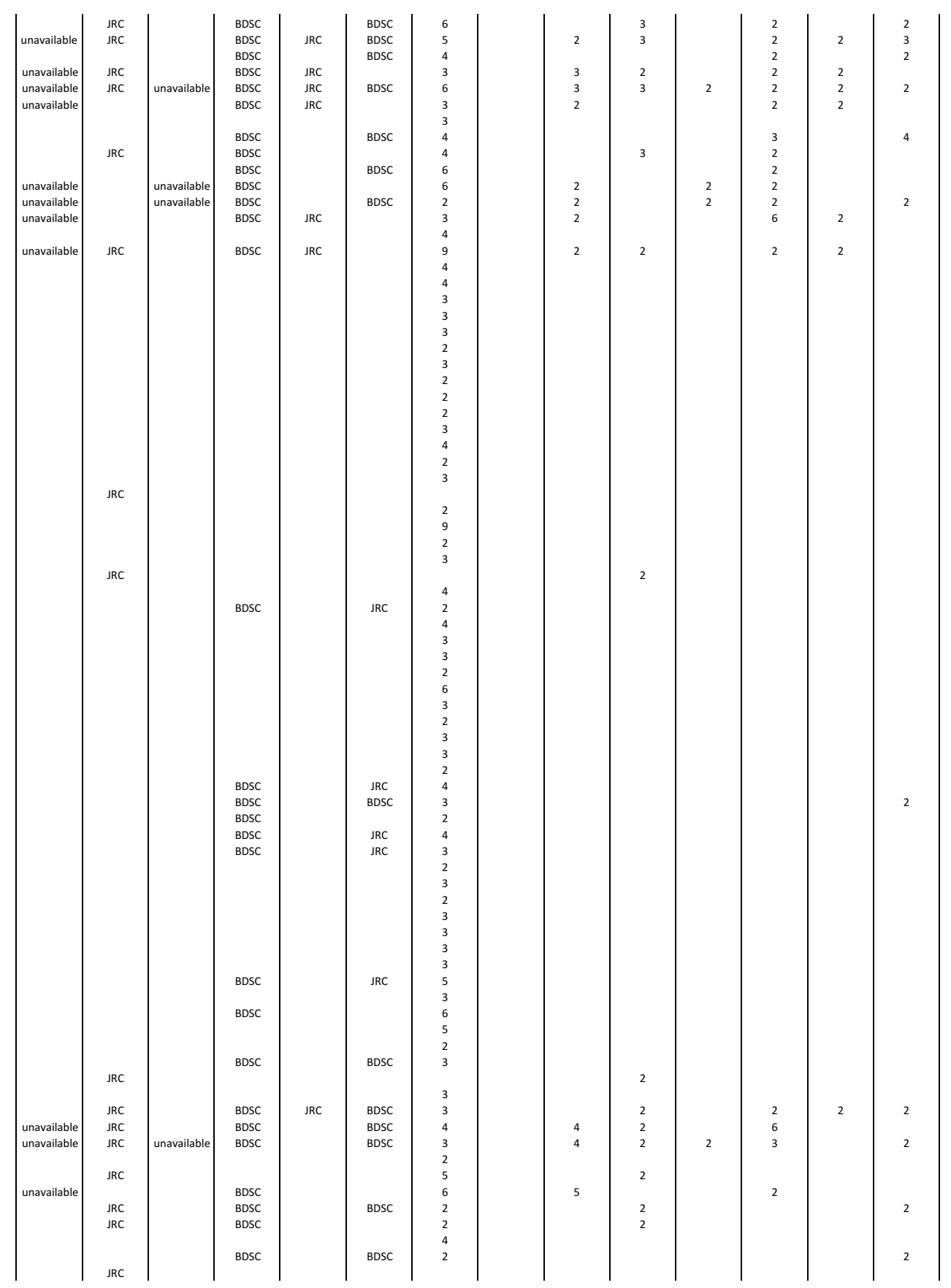


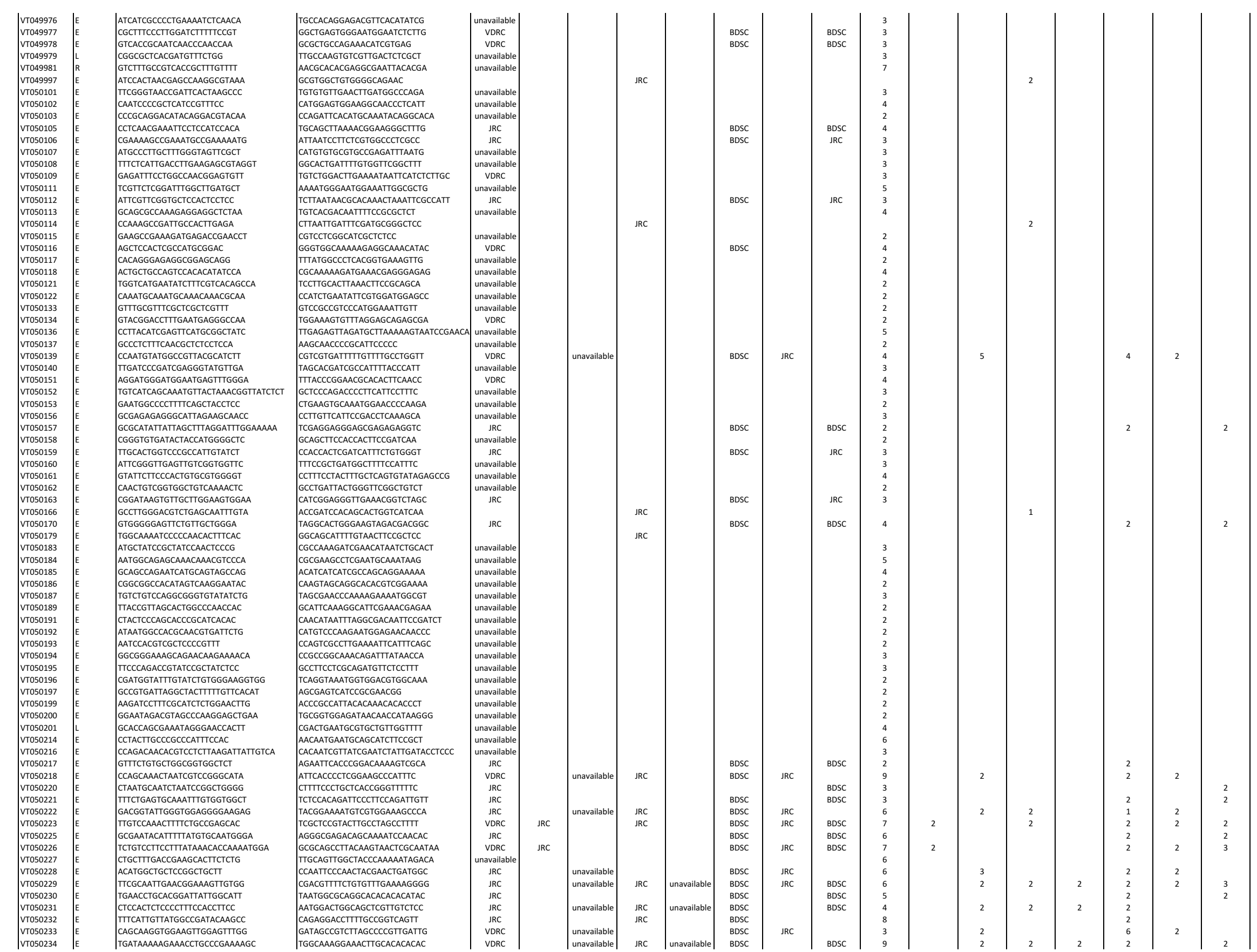




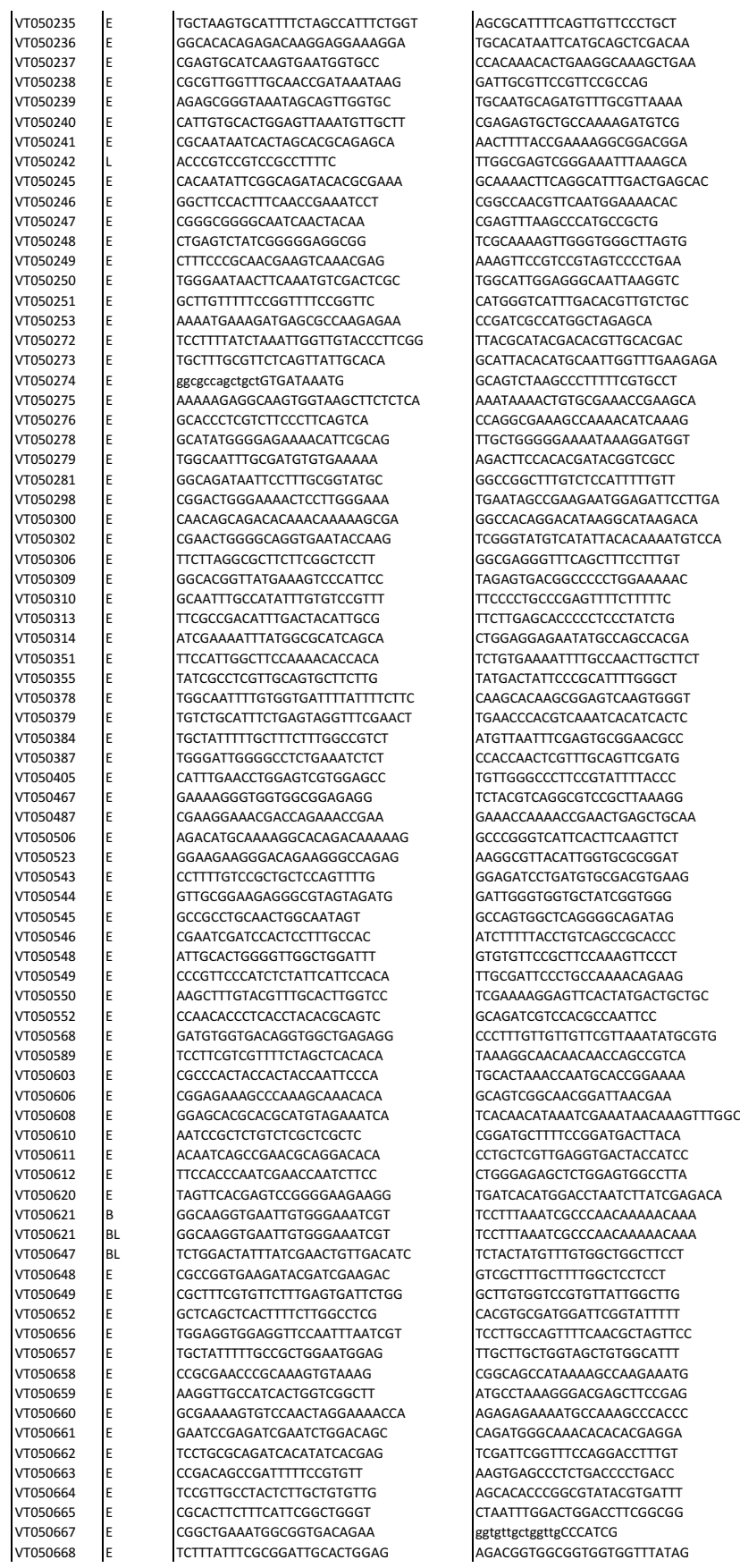

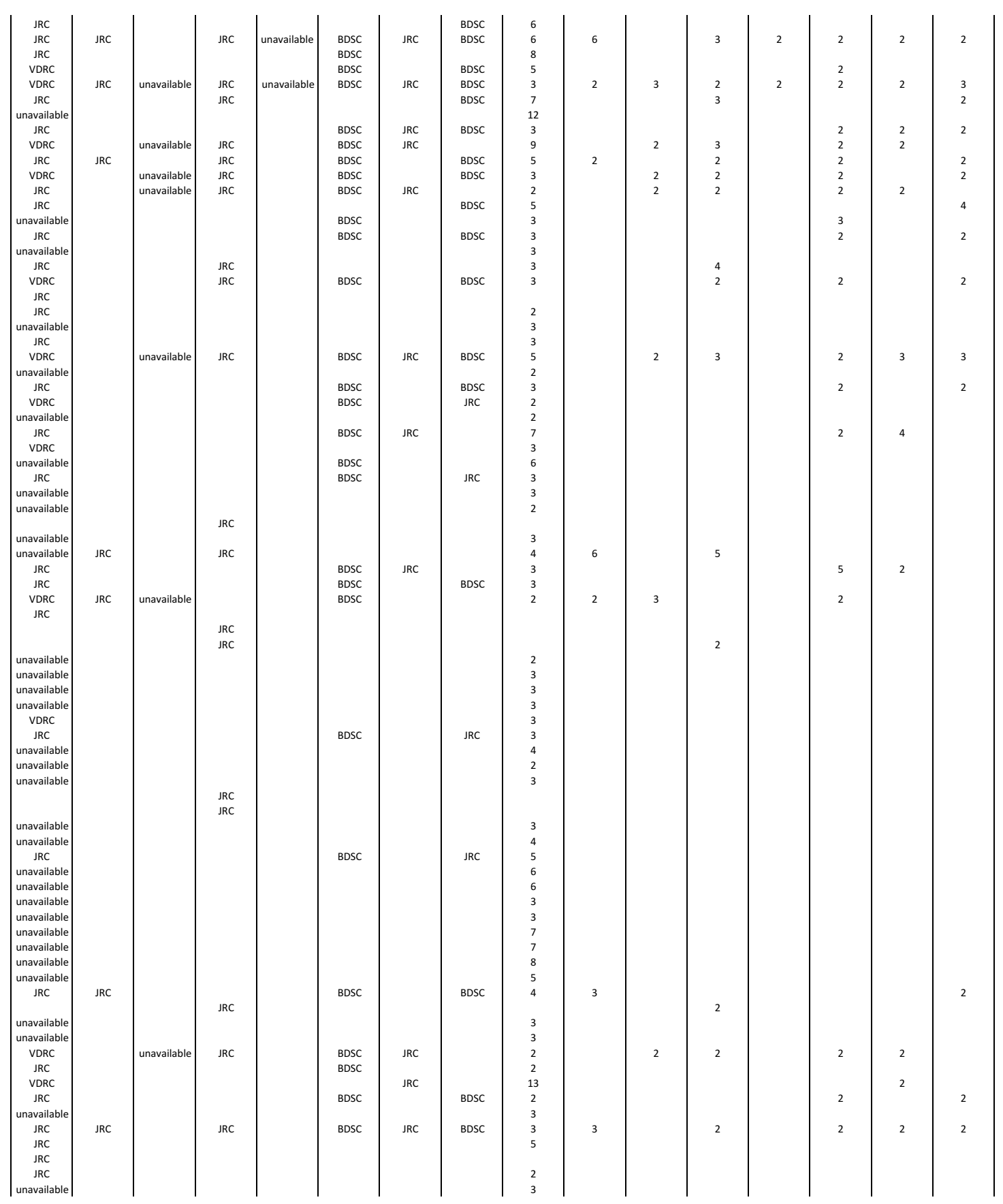




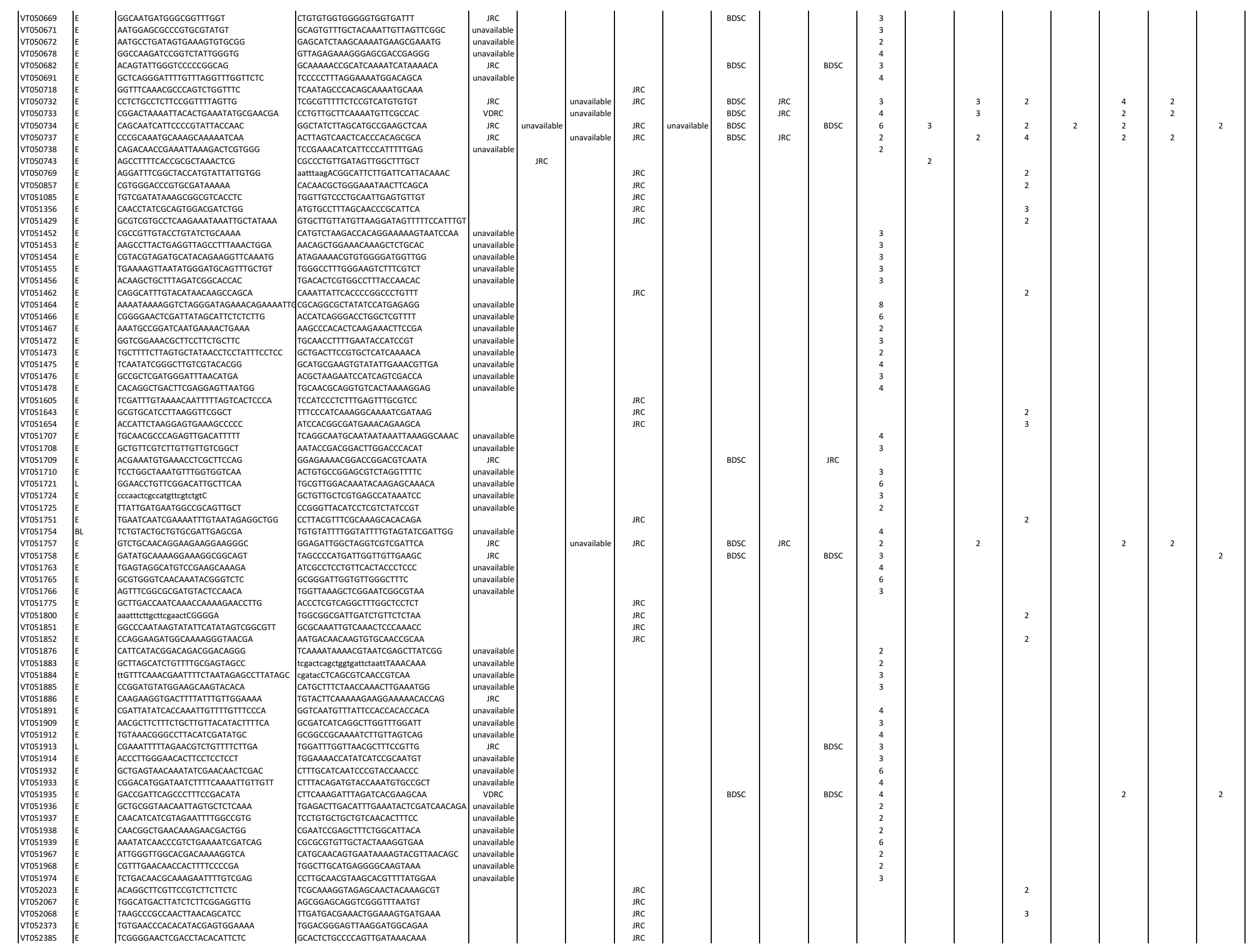




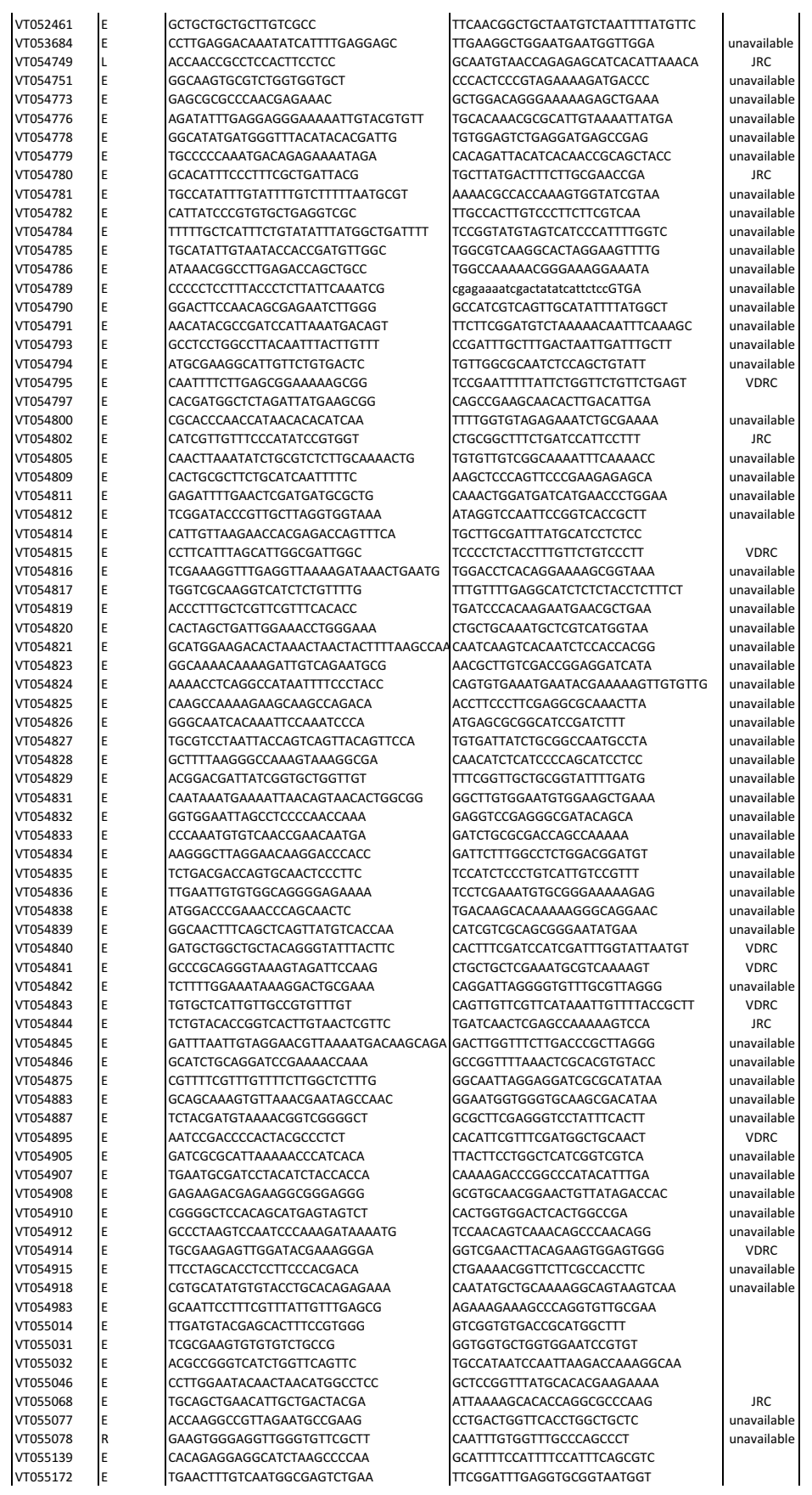

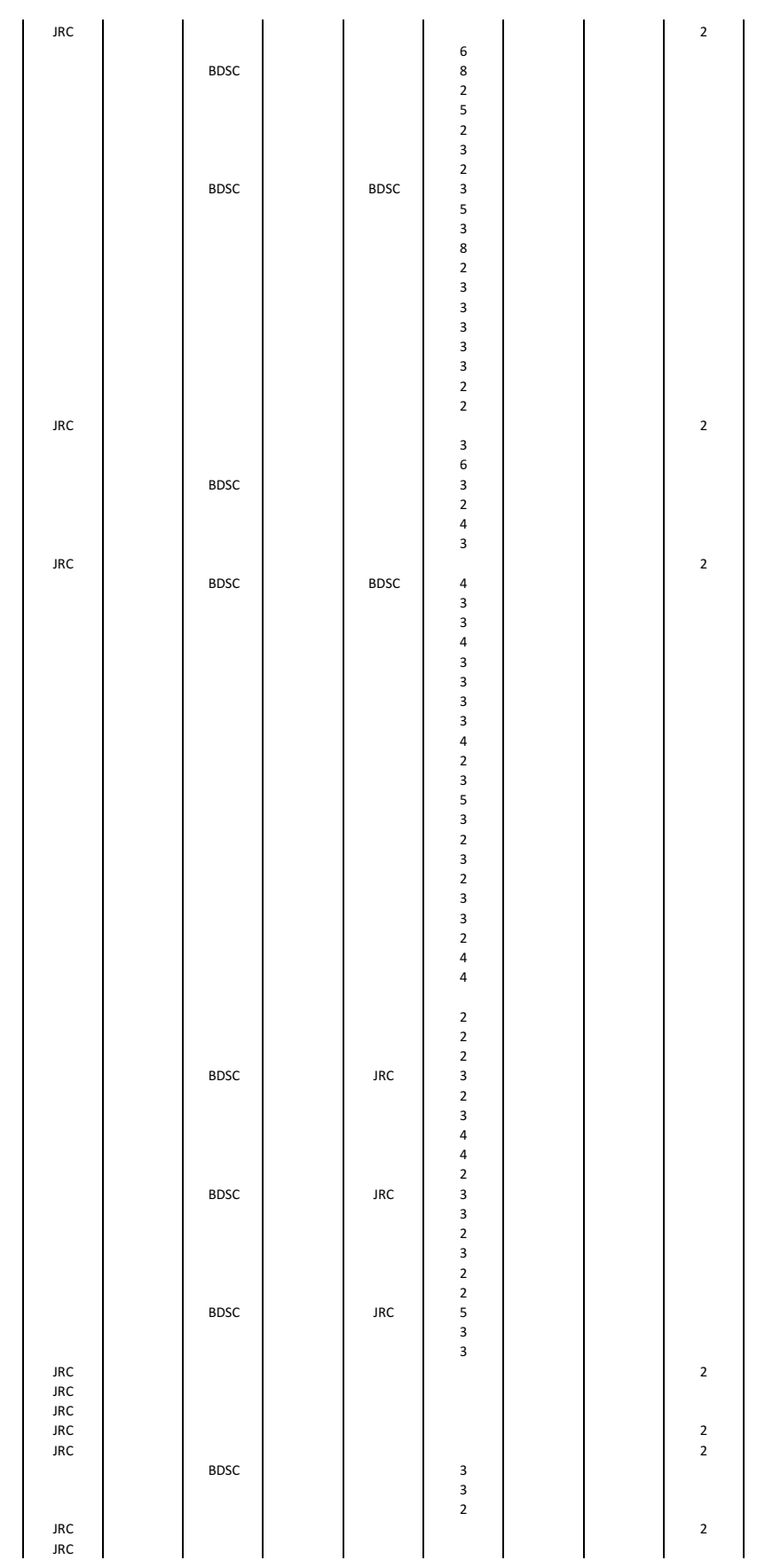




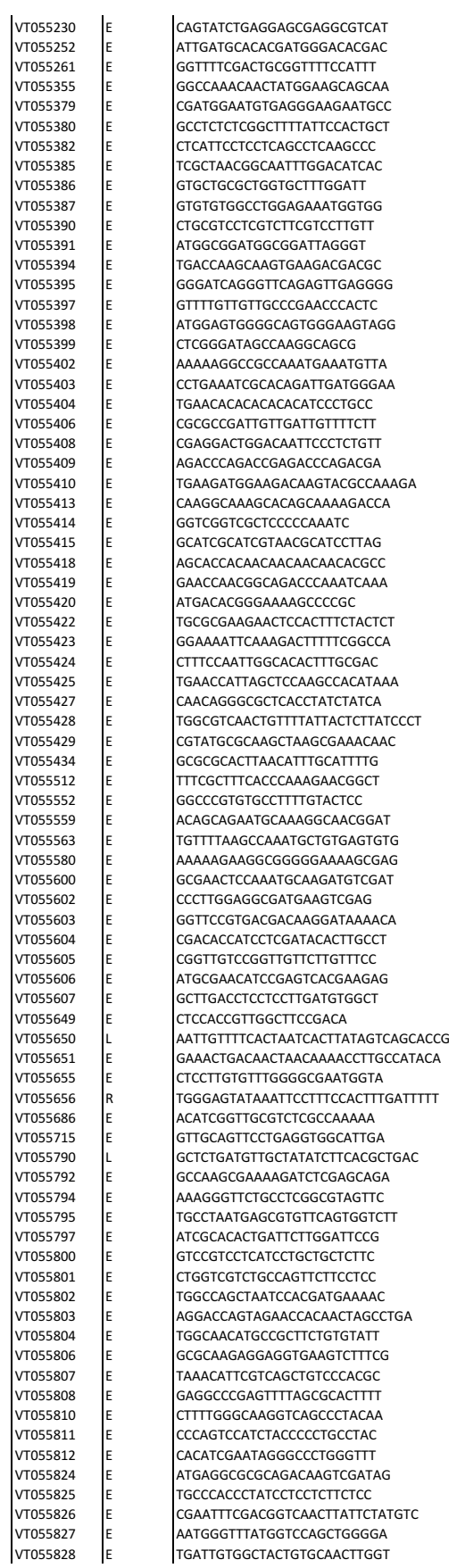

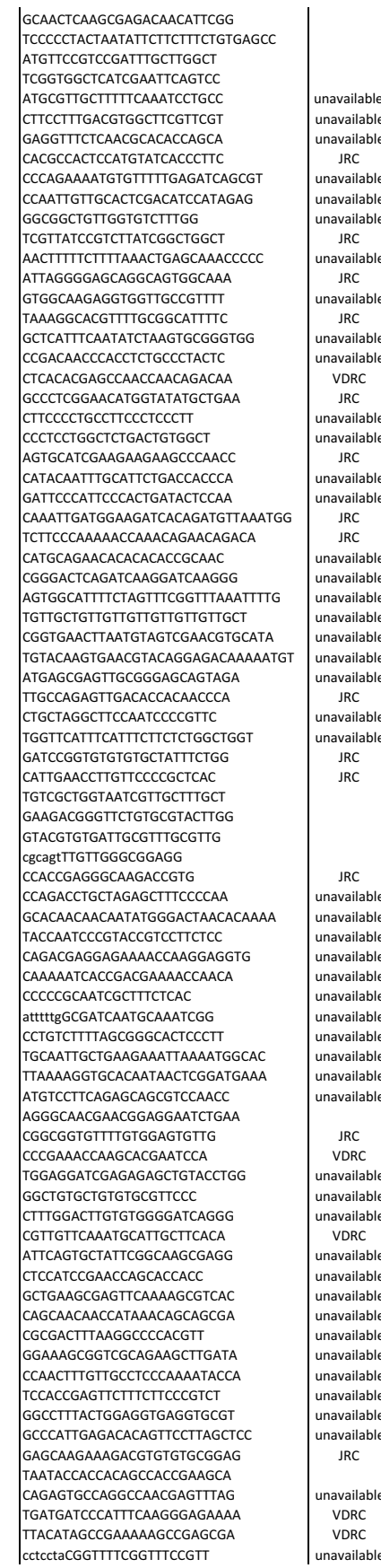
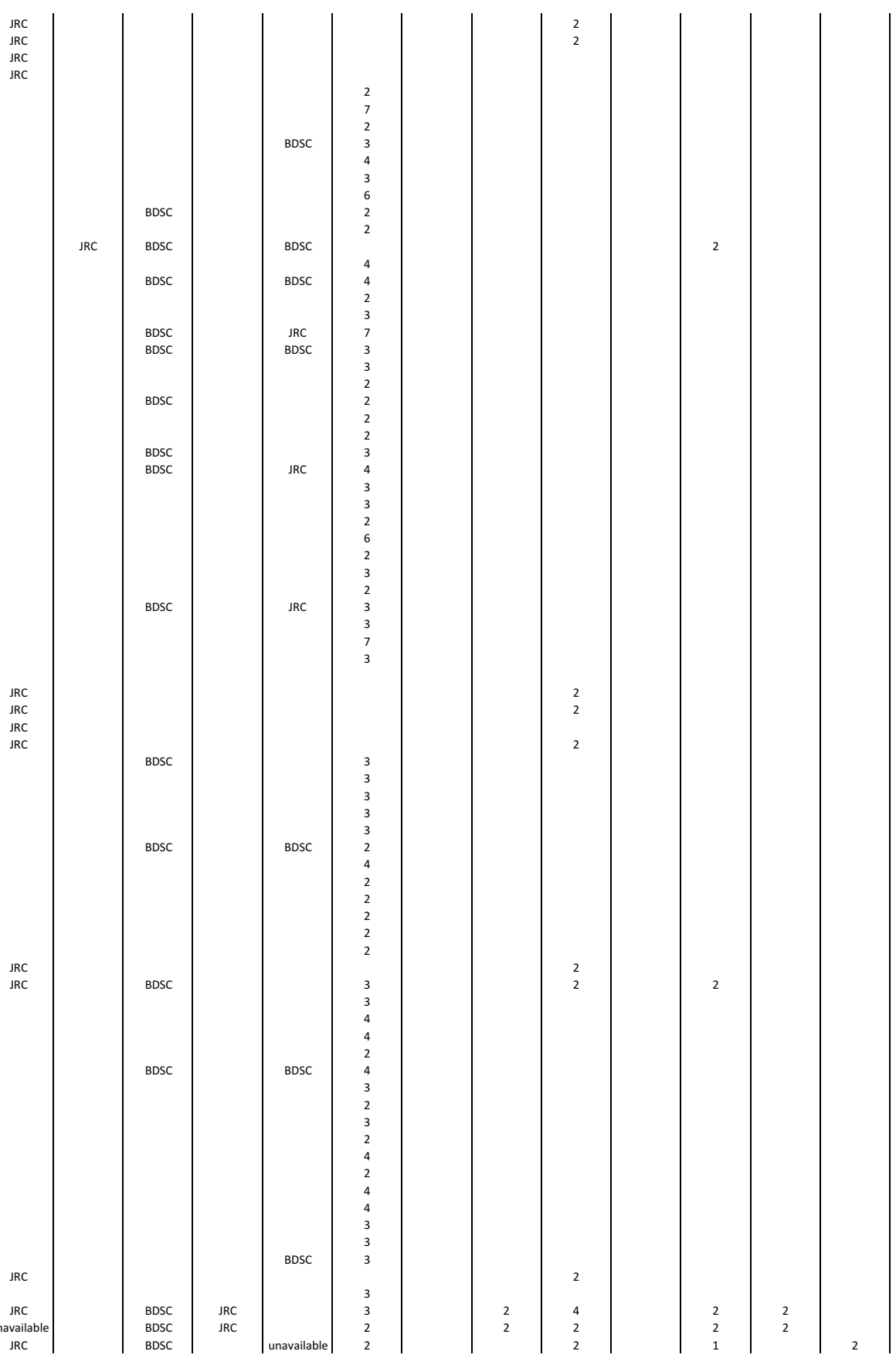


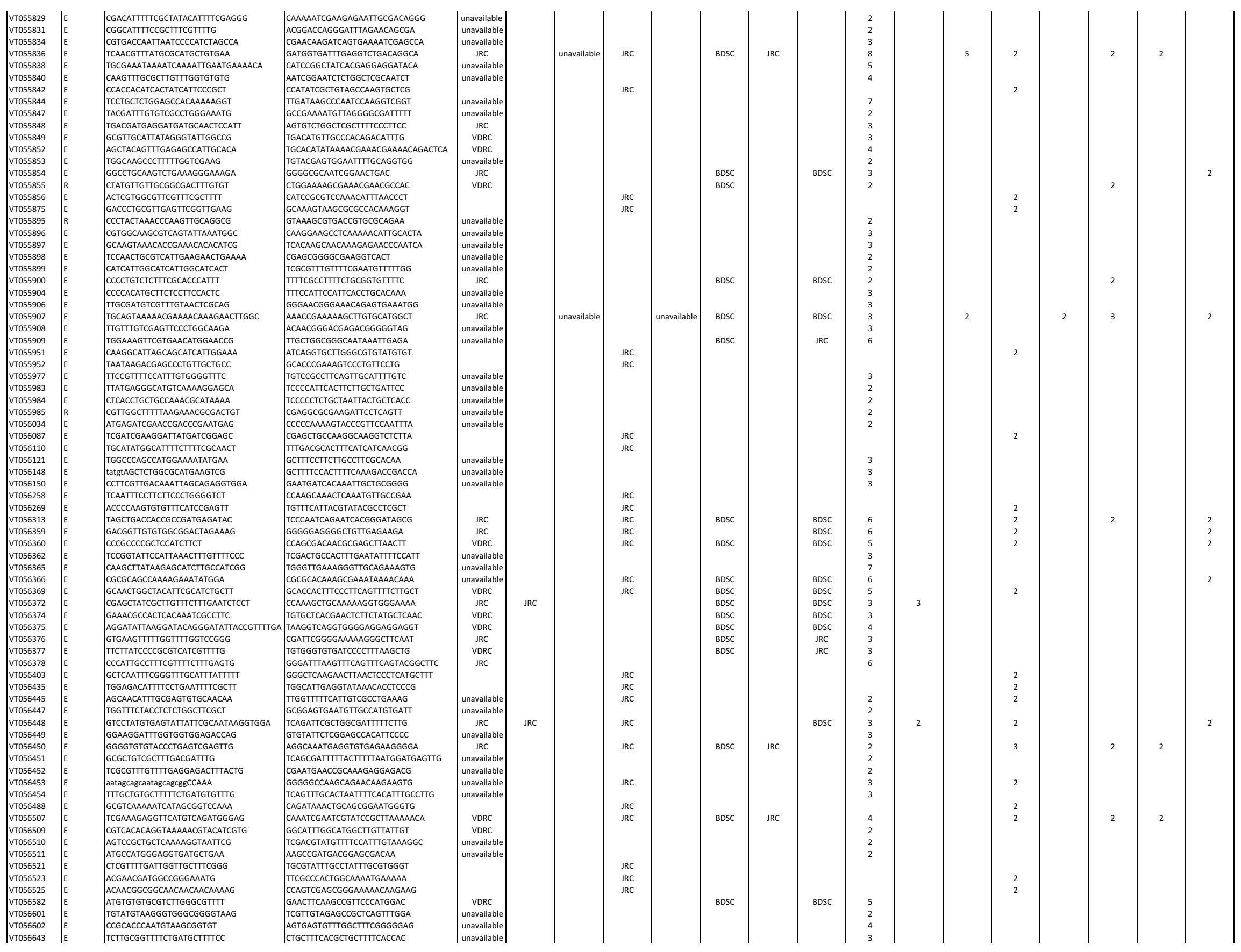




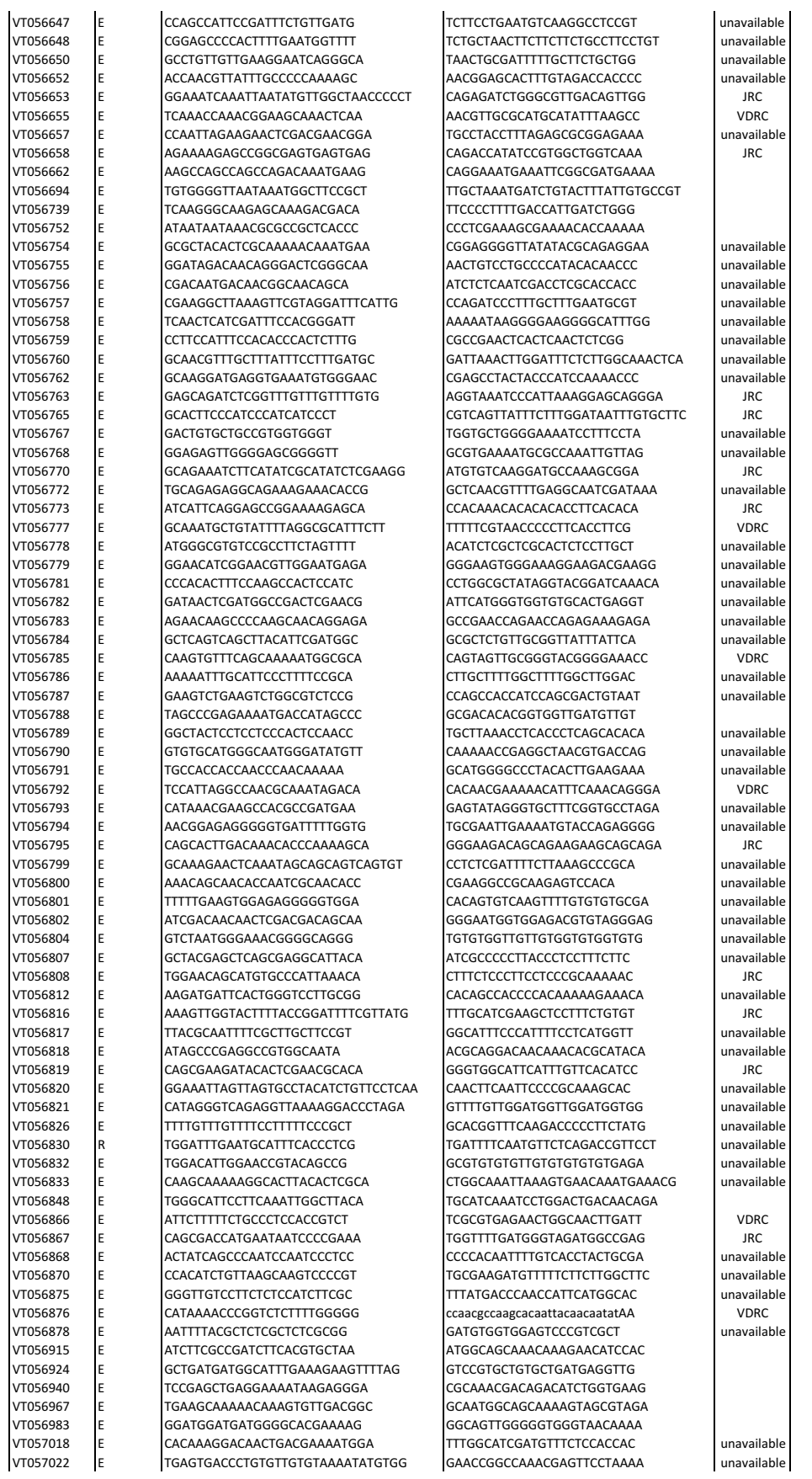

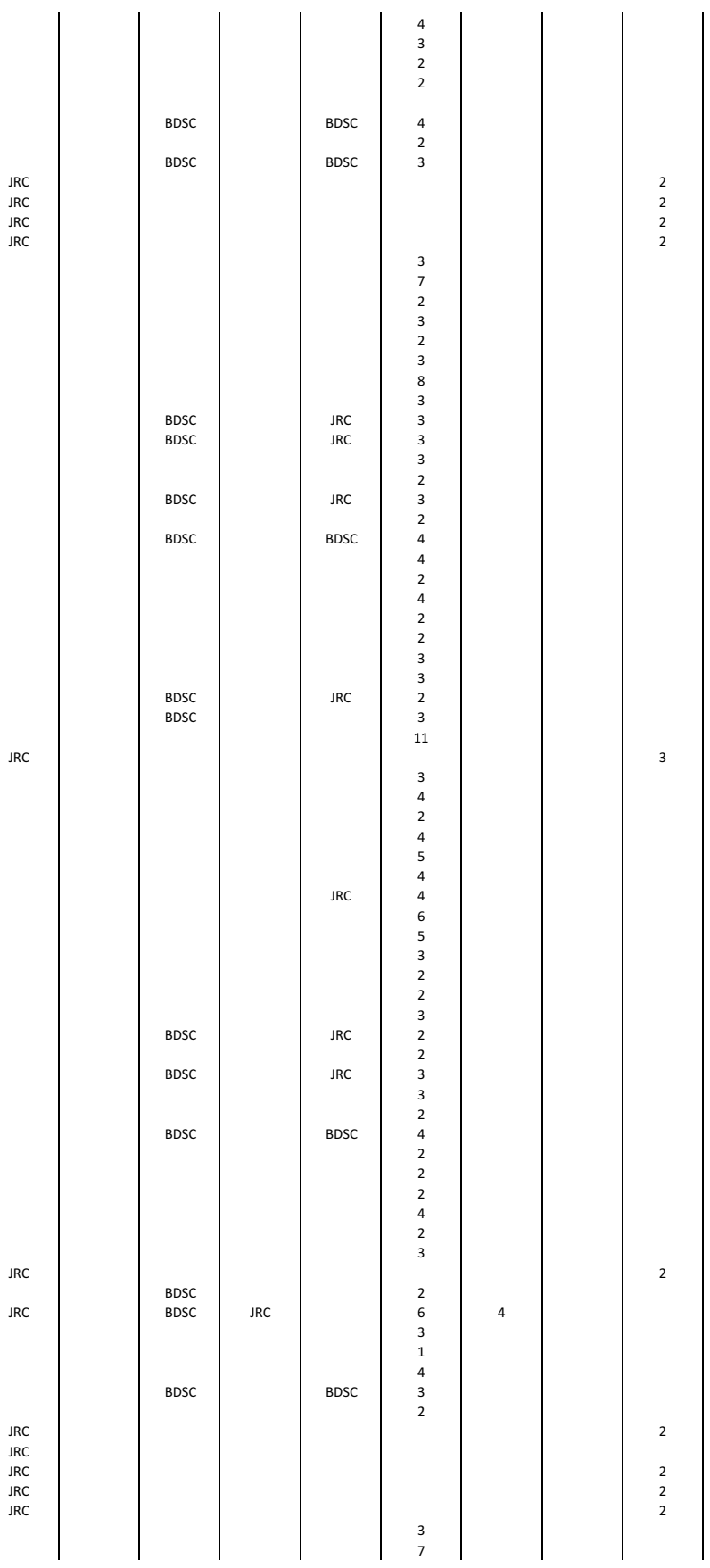




\begin{tabular}{|c|c|c|c|}
\hline & \multicolumn{2}{|r|}{ 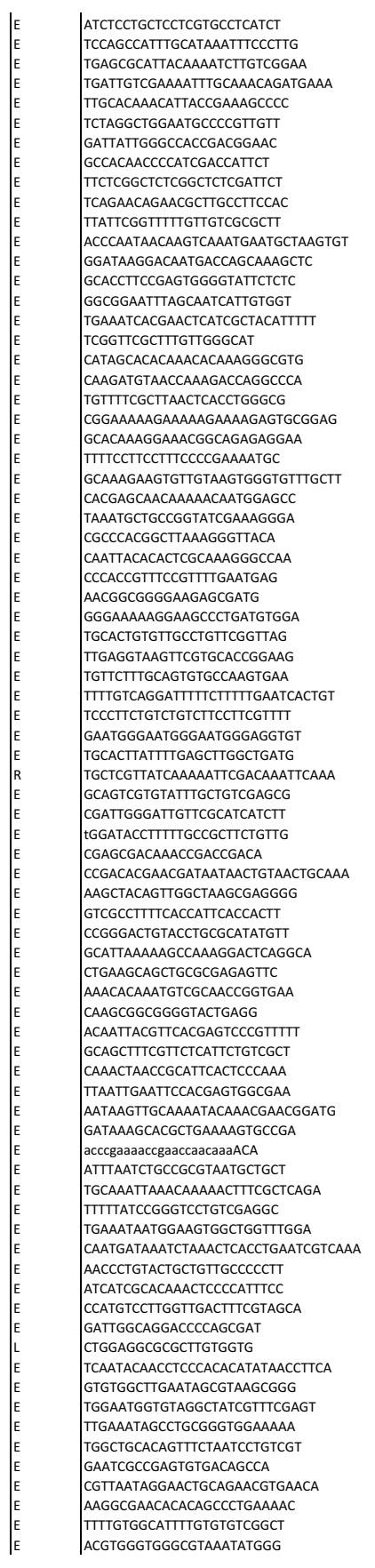 } & 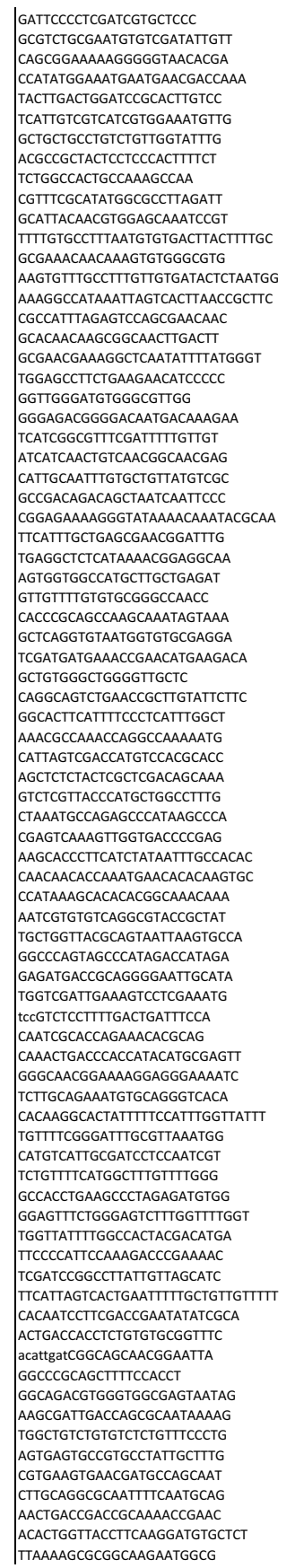 \\
\hline
\end{tabular}
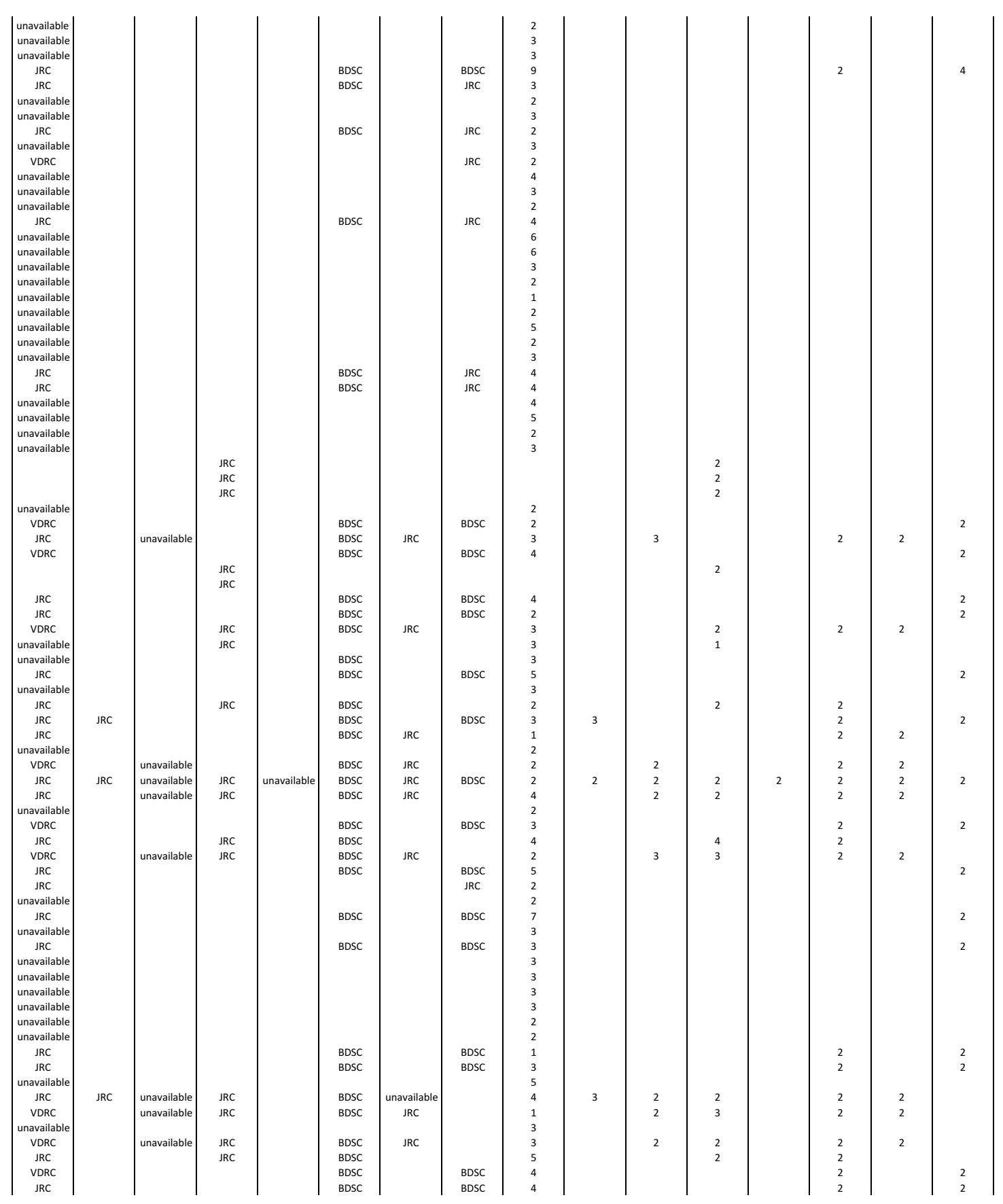


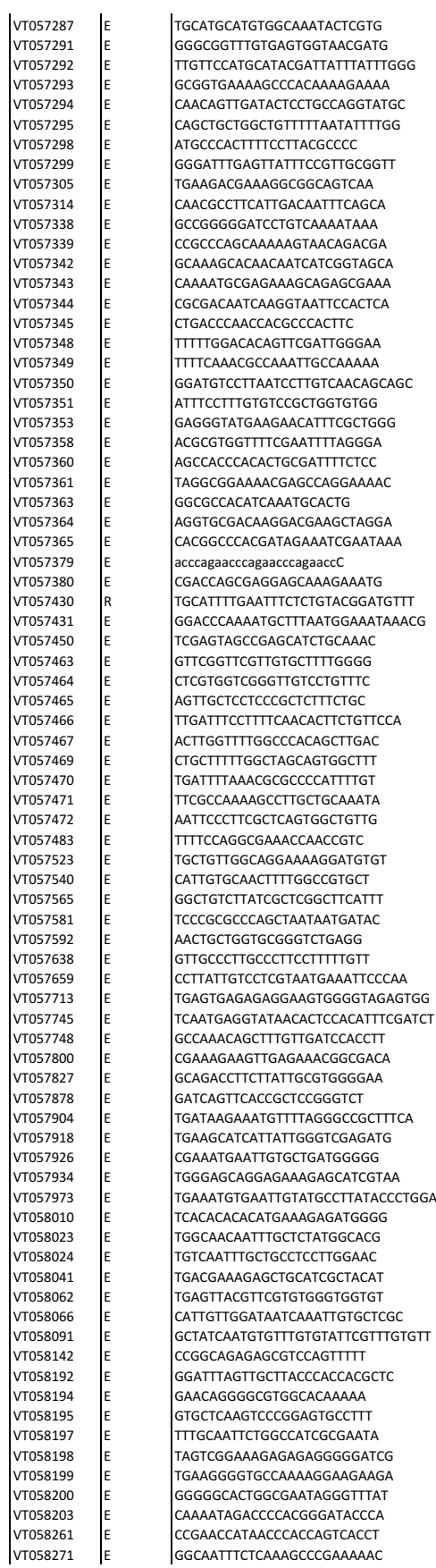

\begin{tabular}{|c|}
\hline 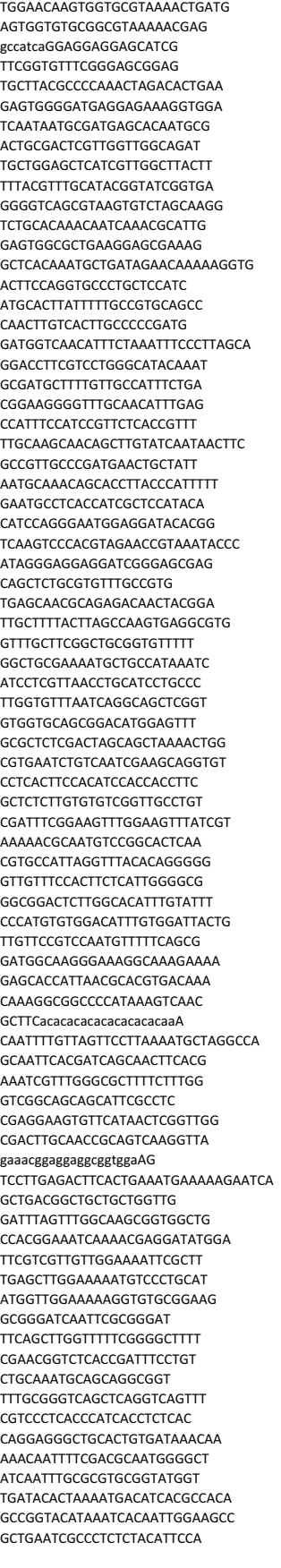 \\
\hline
\end{tabular}
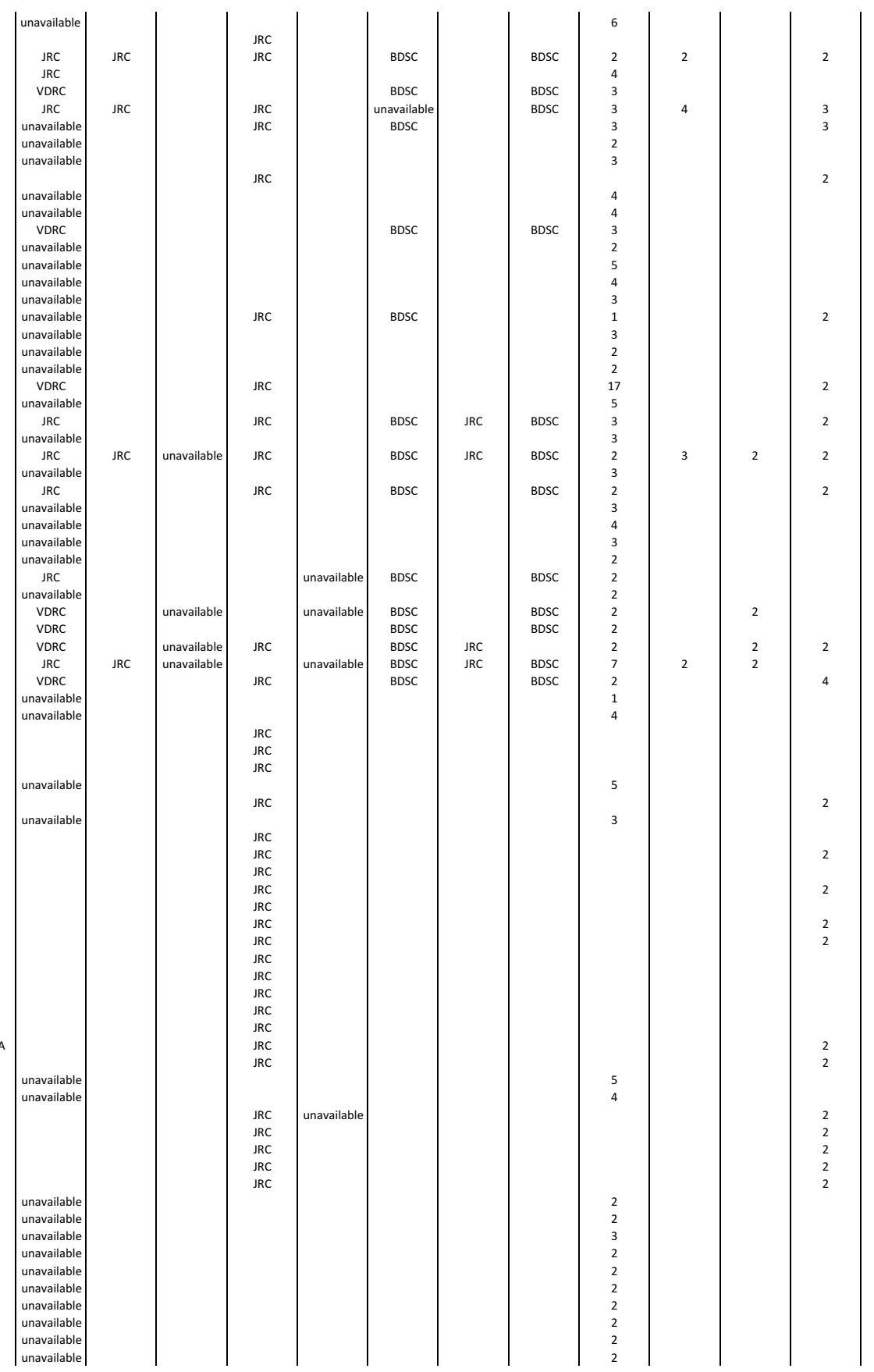


\begin{tabular}{|c|c|c|c|}
\hline & 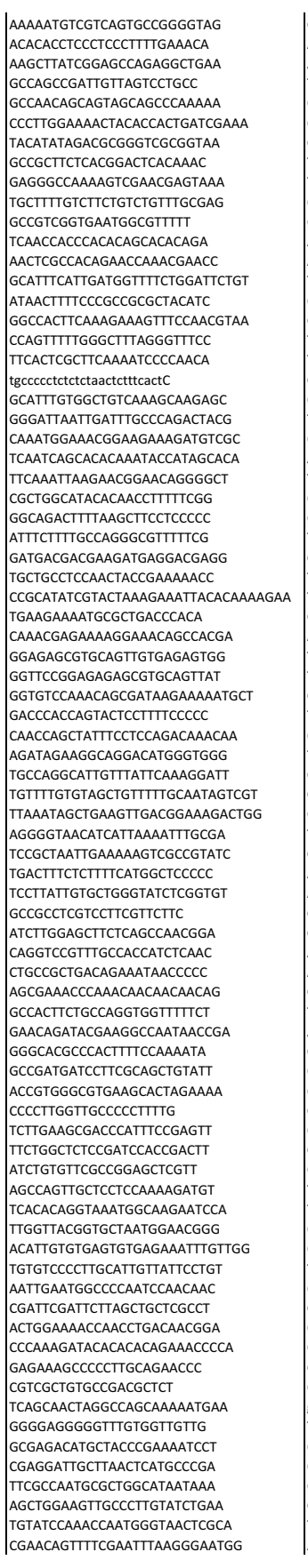 & 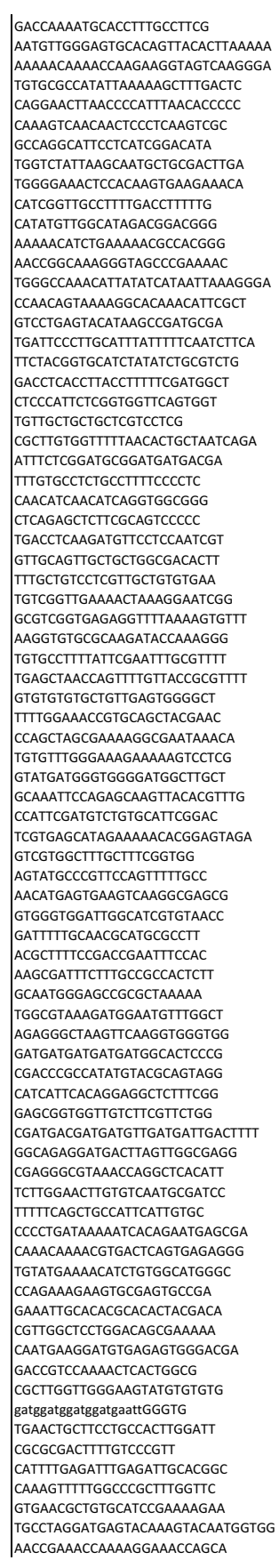 \\
\hline
\end{tabular}
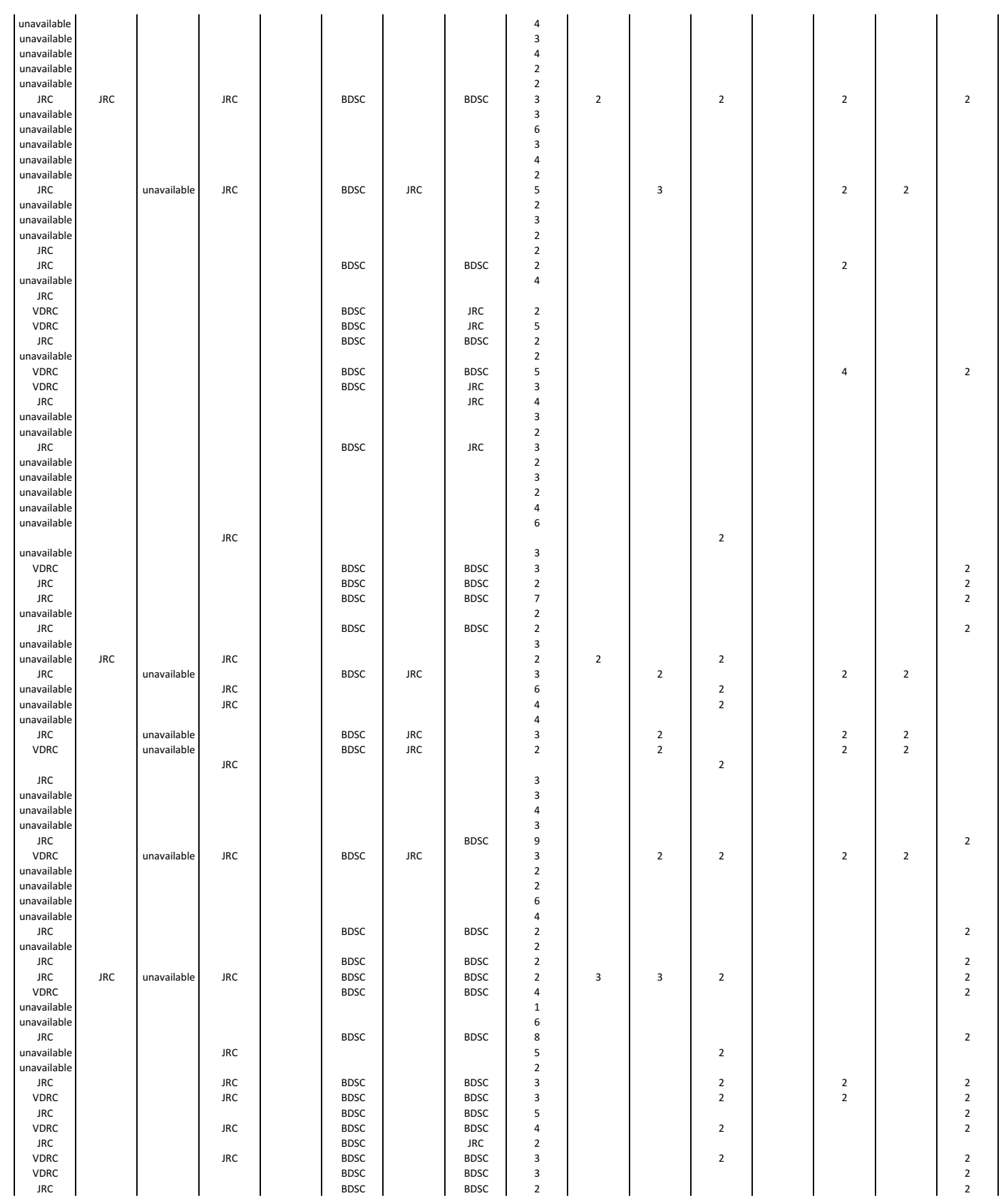


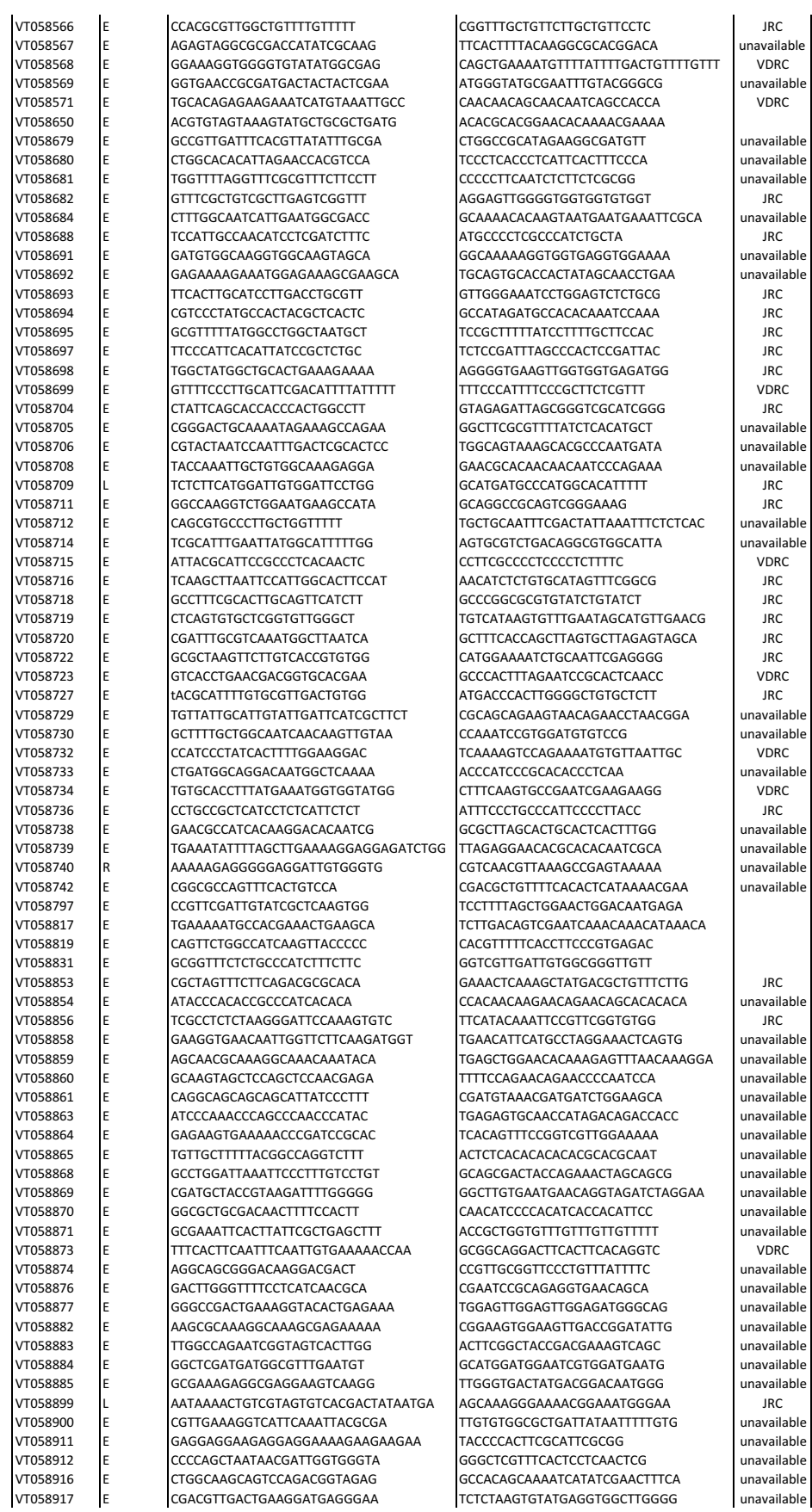
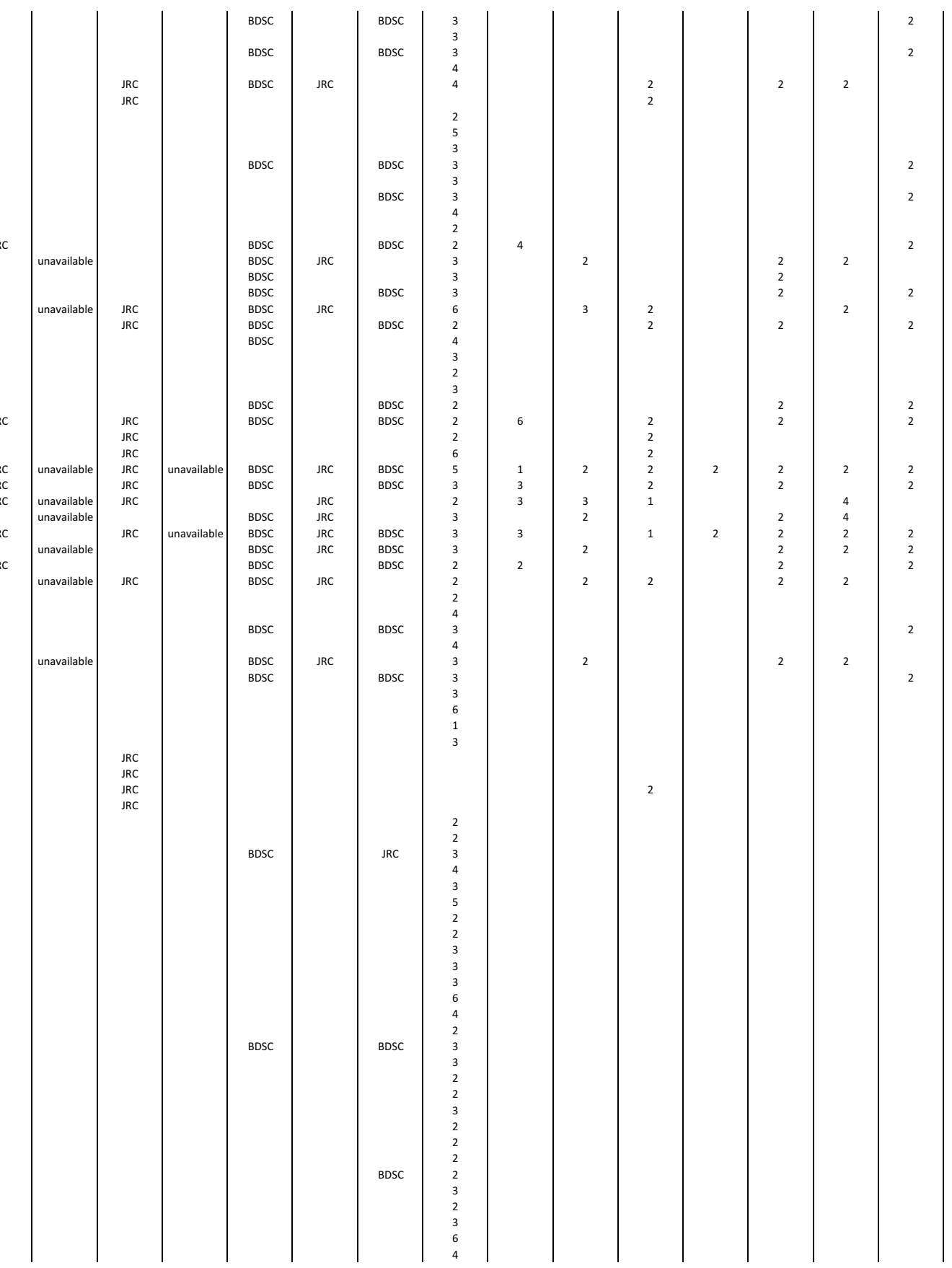


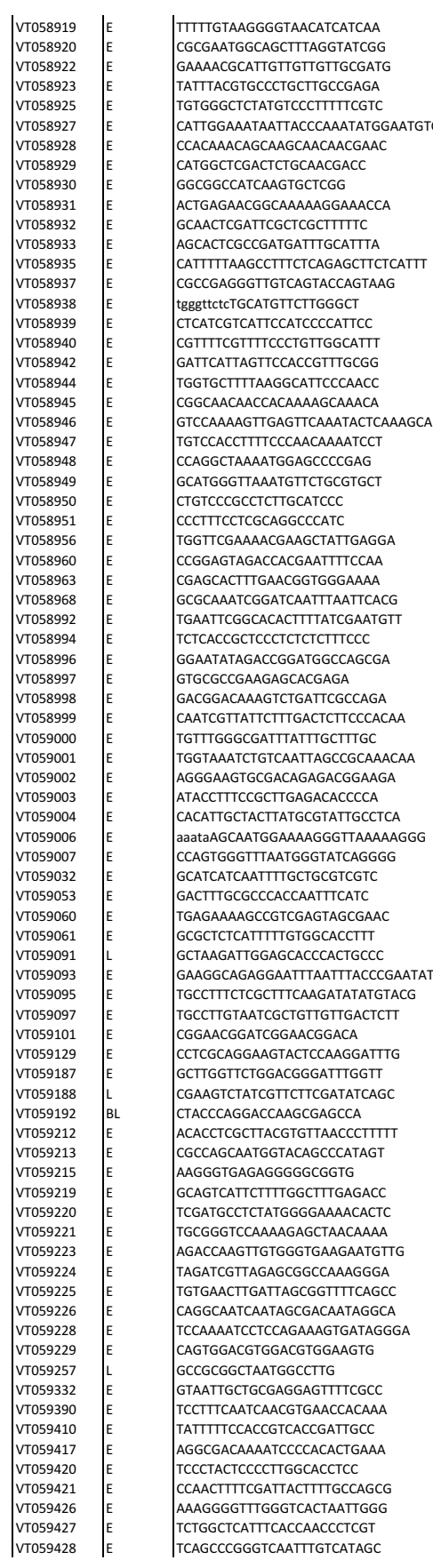

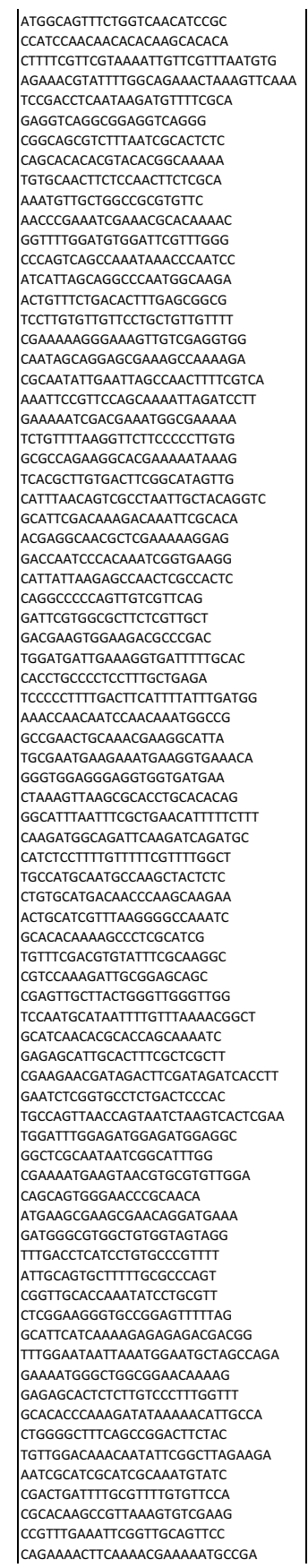
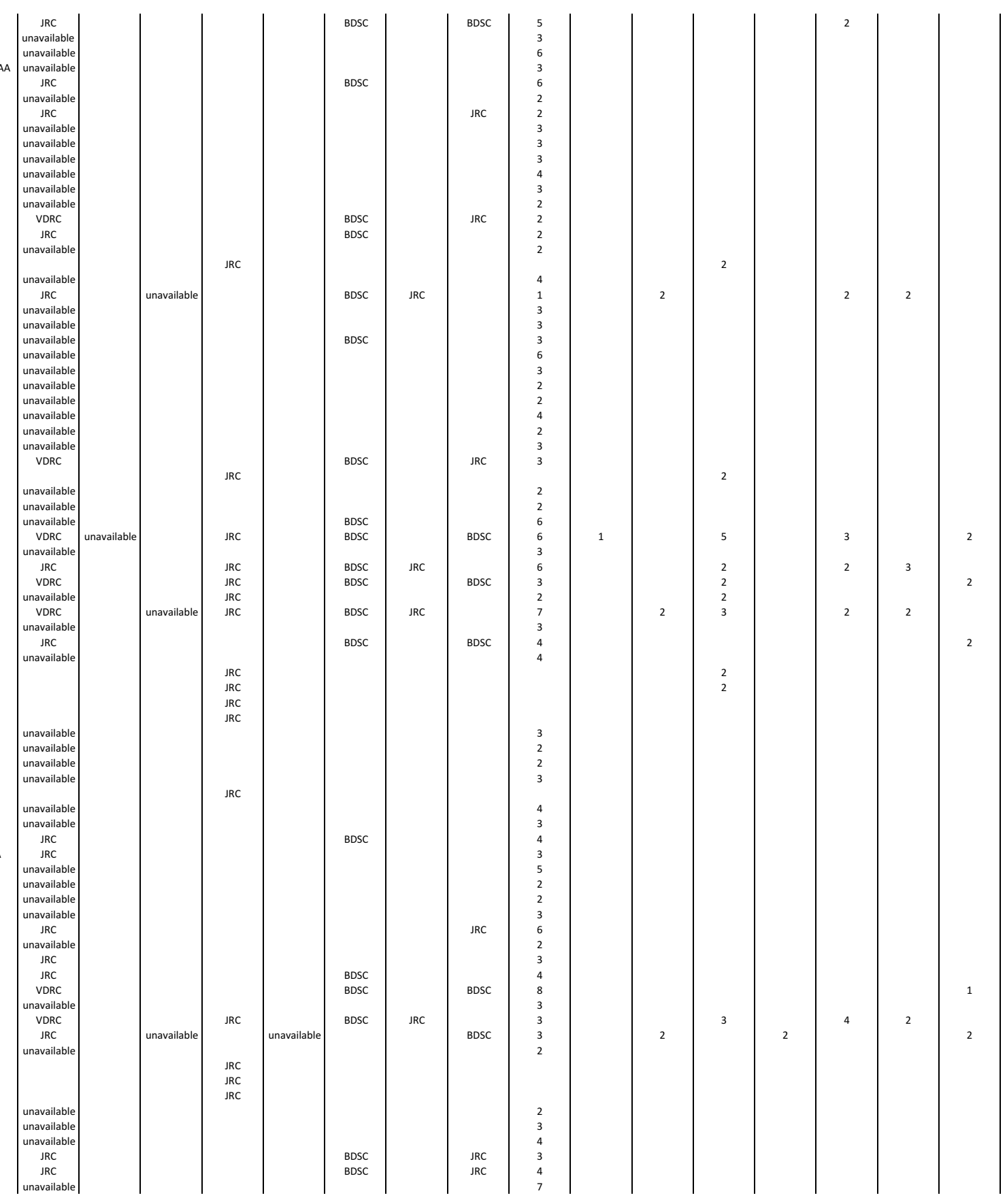


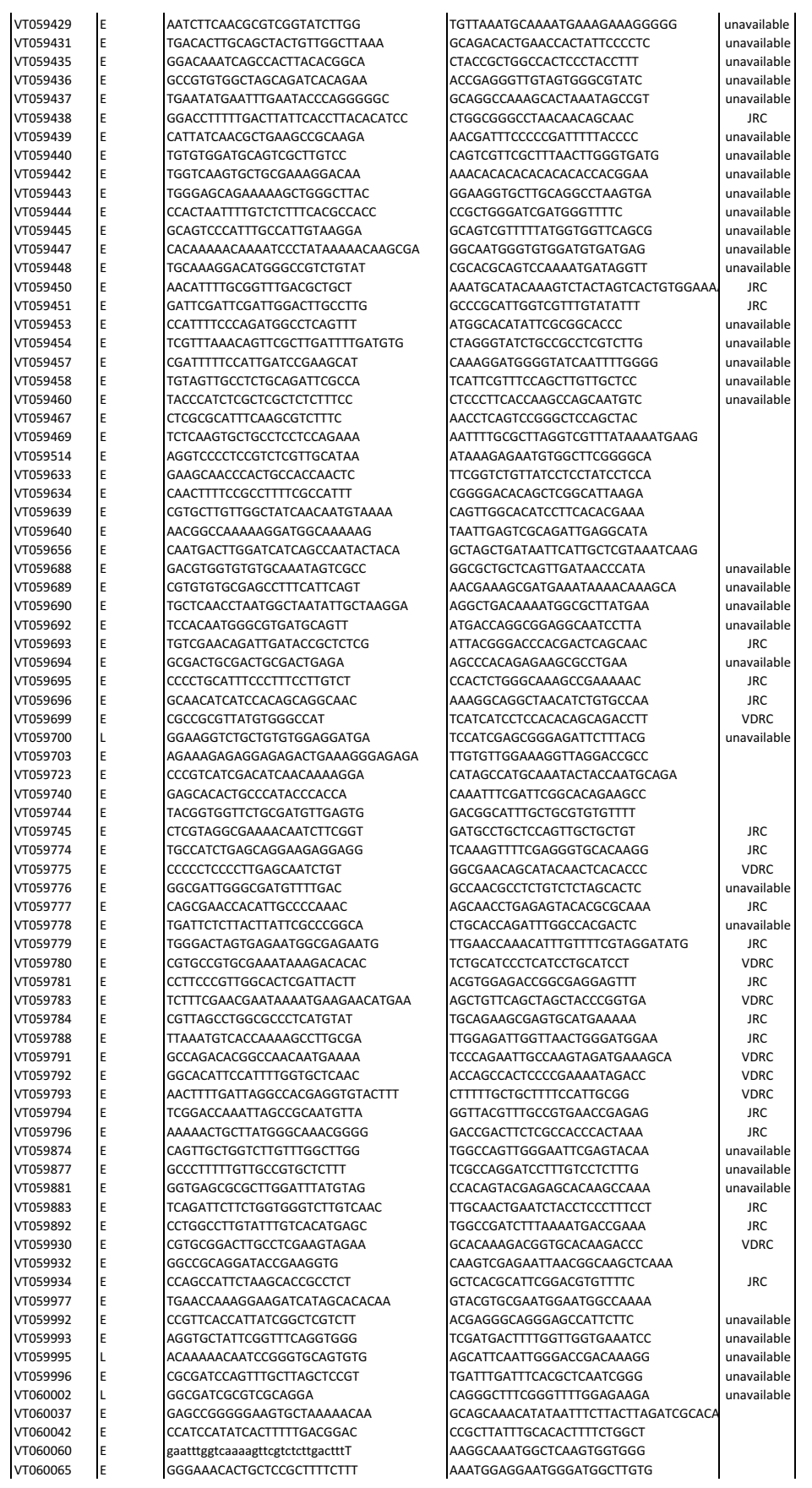
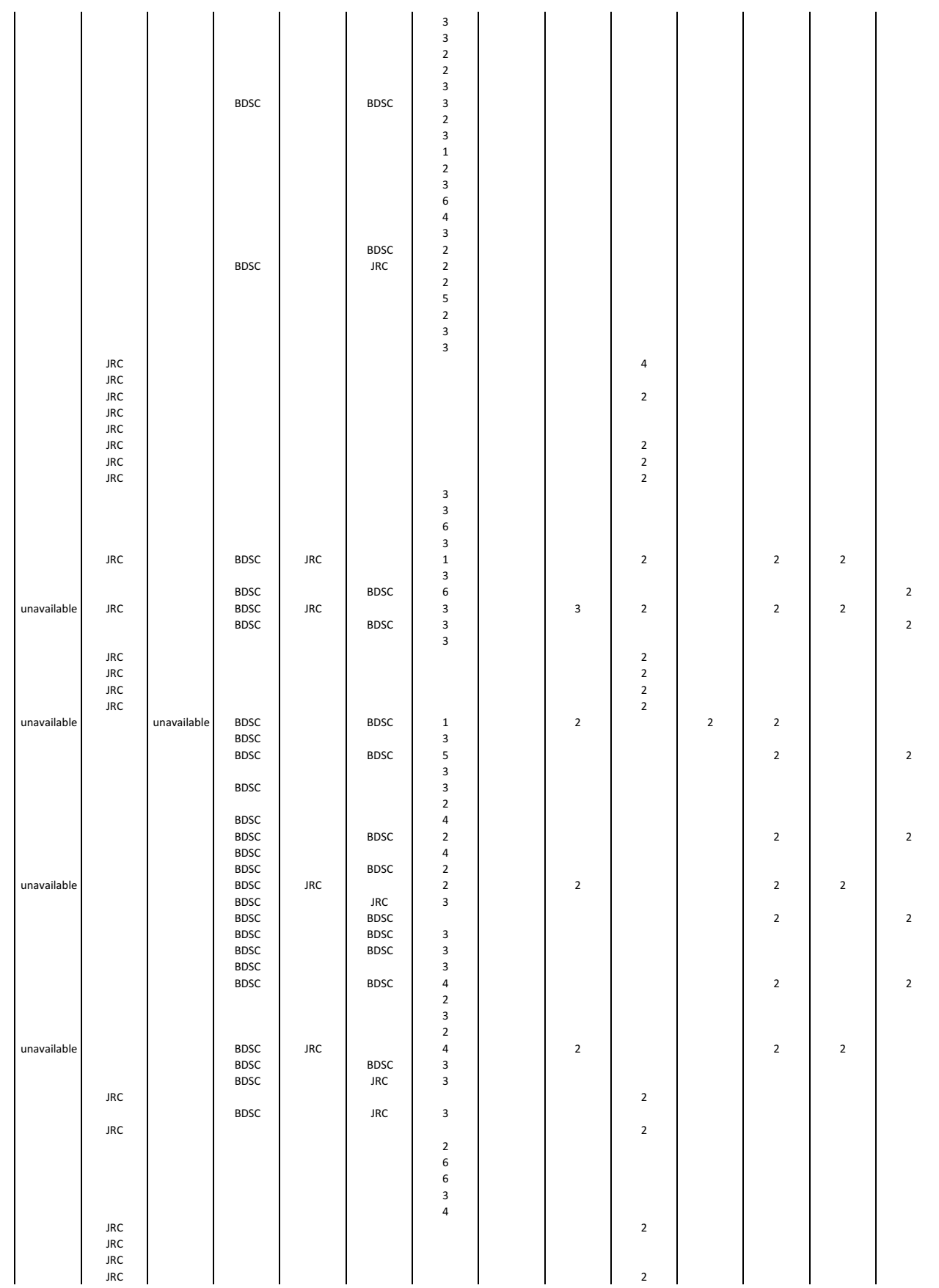


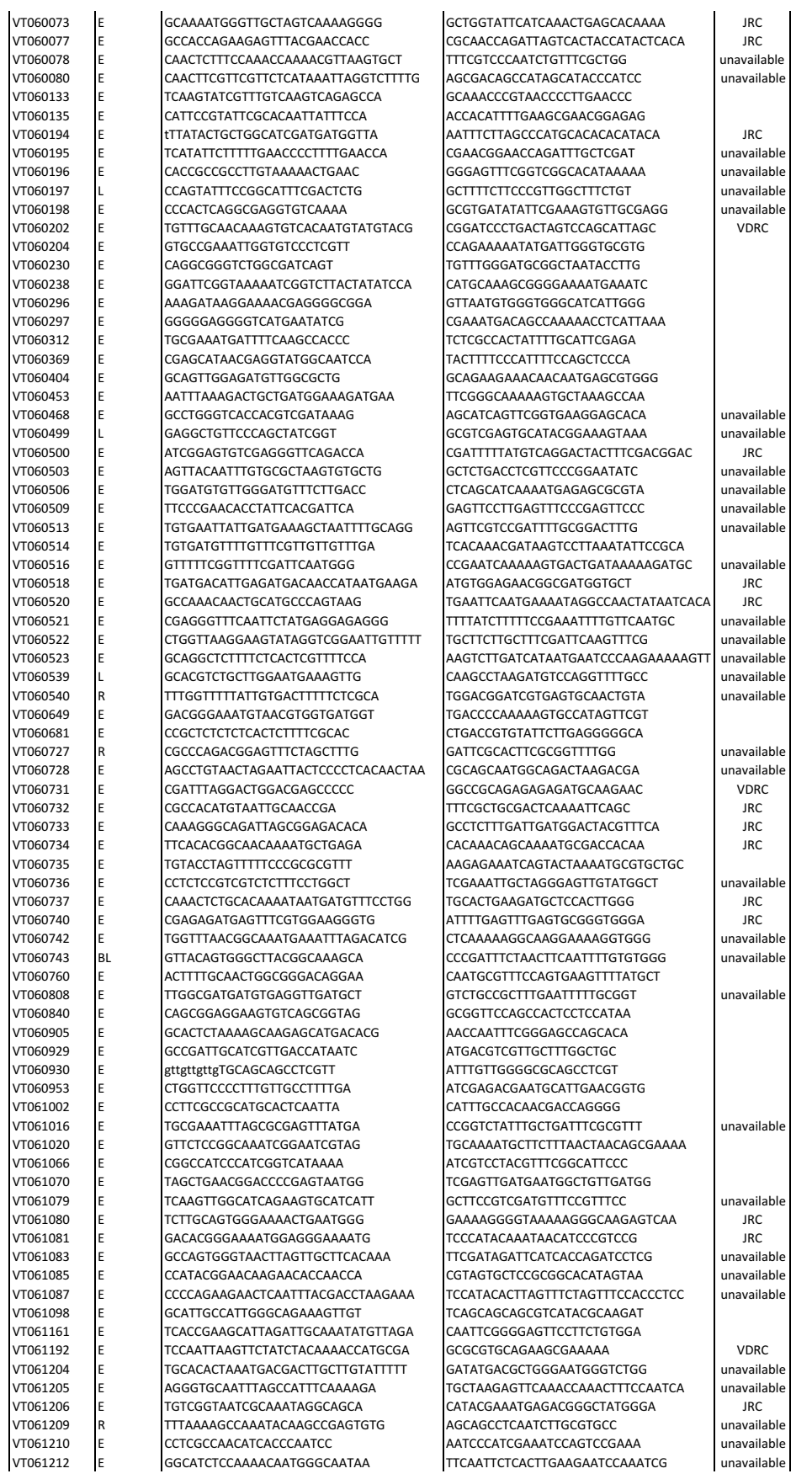
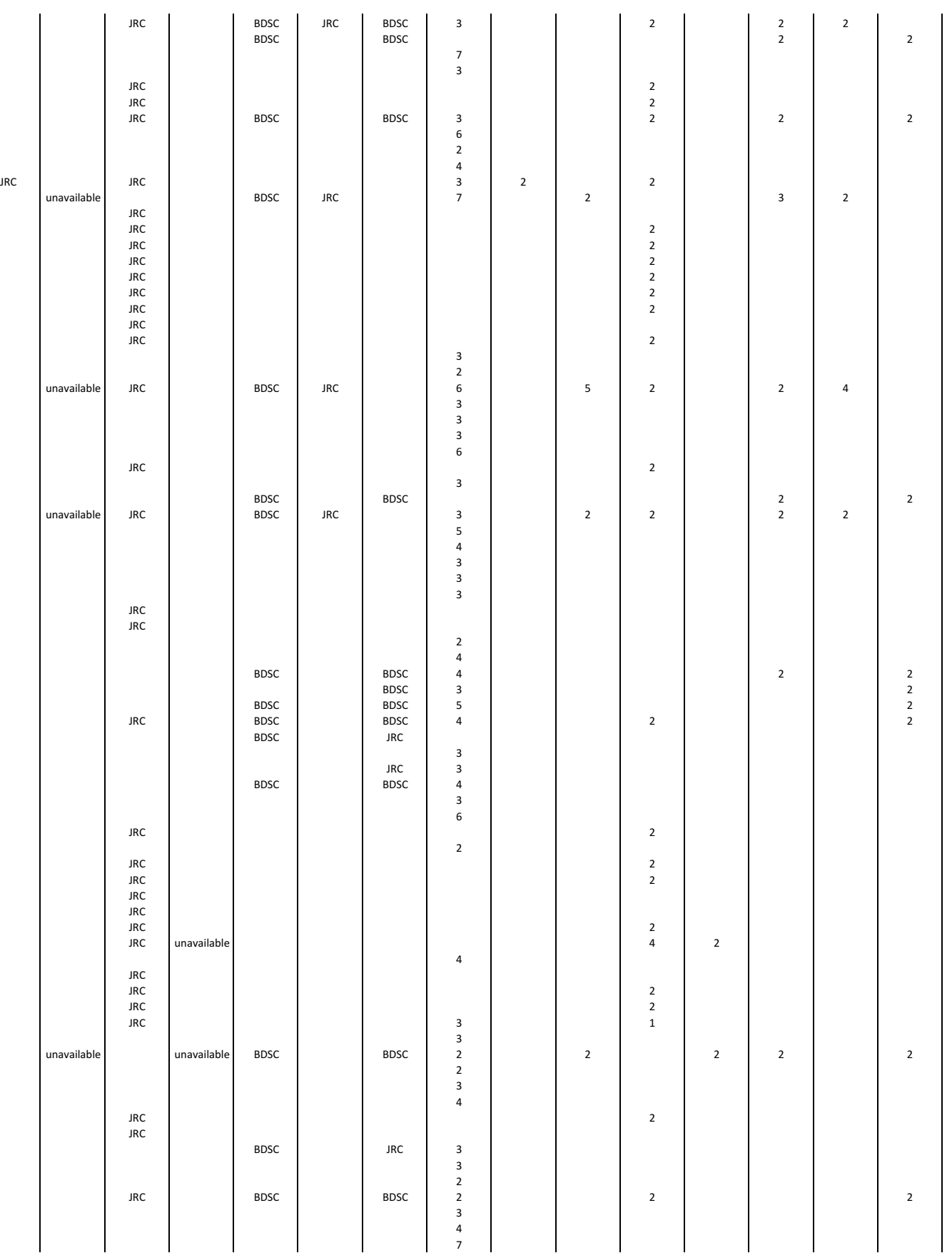


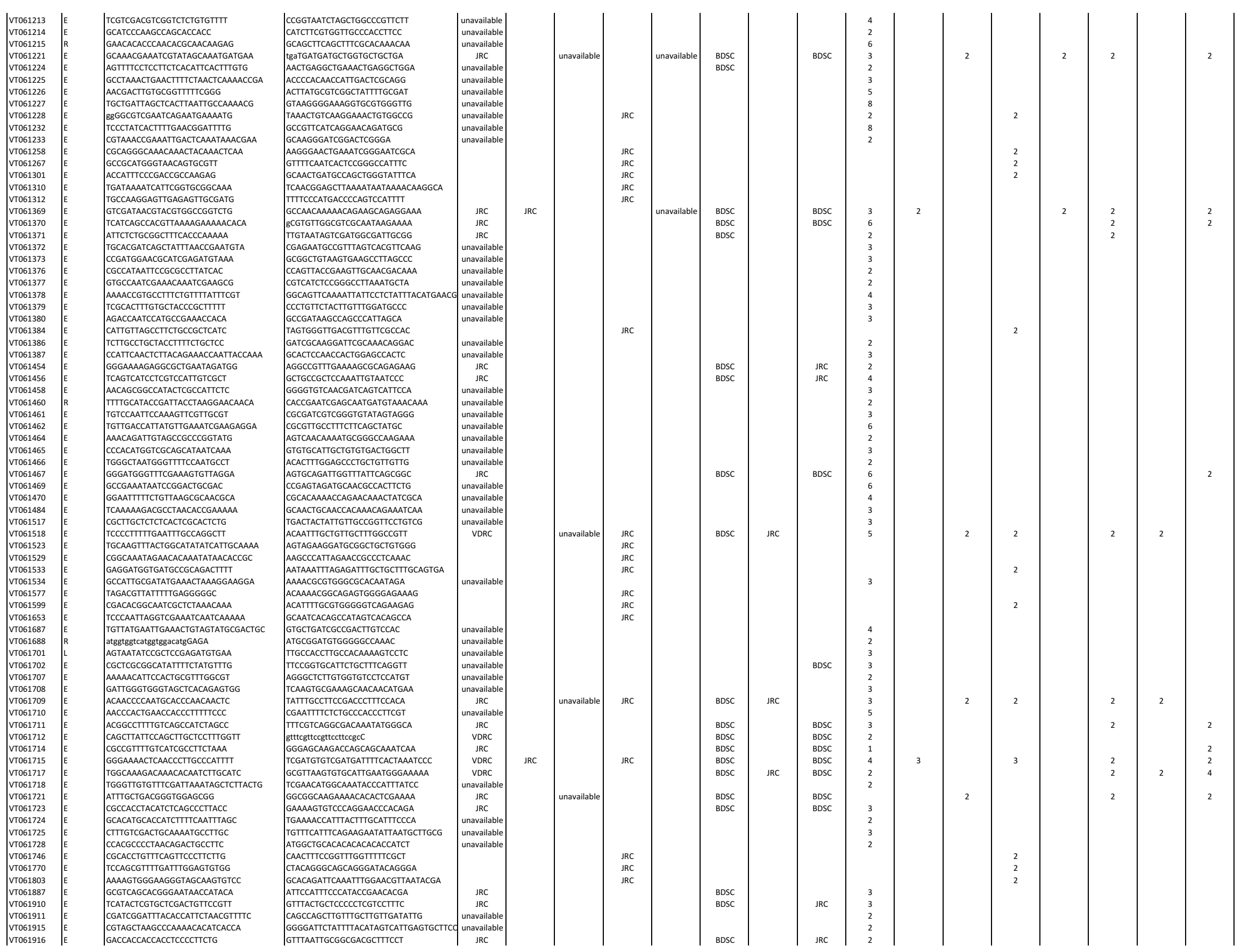




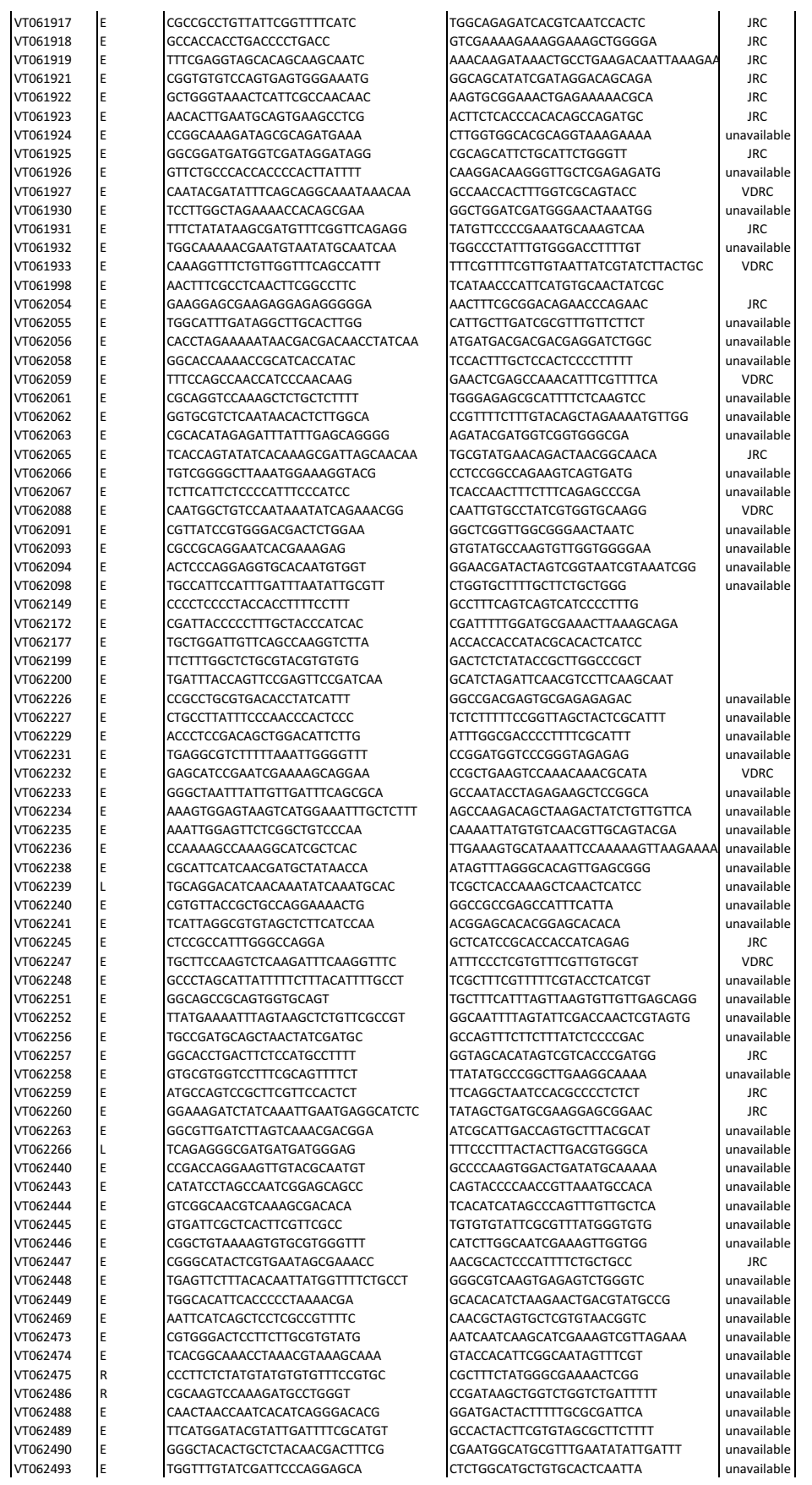
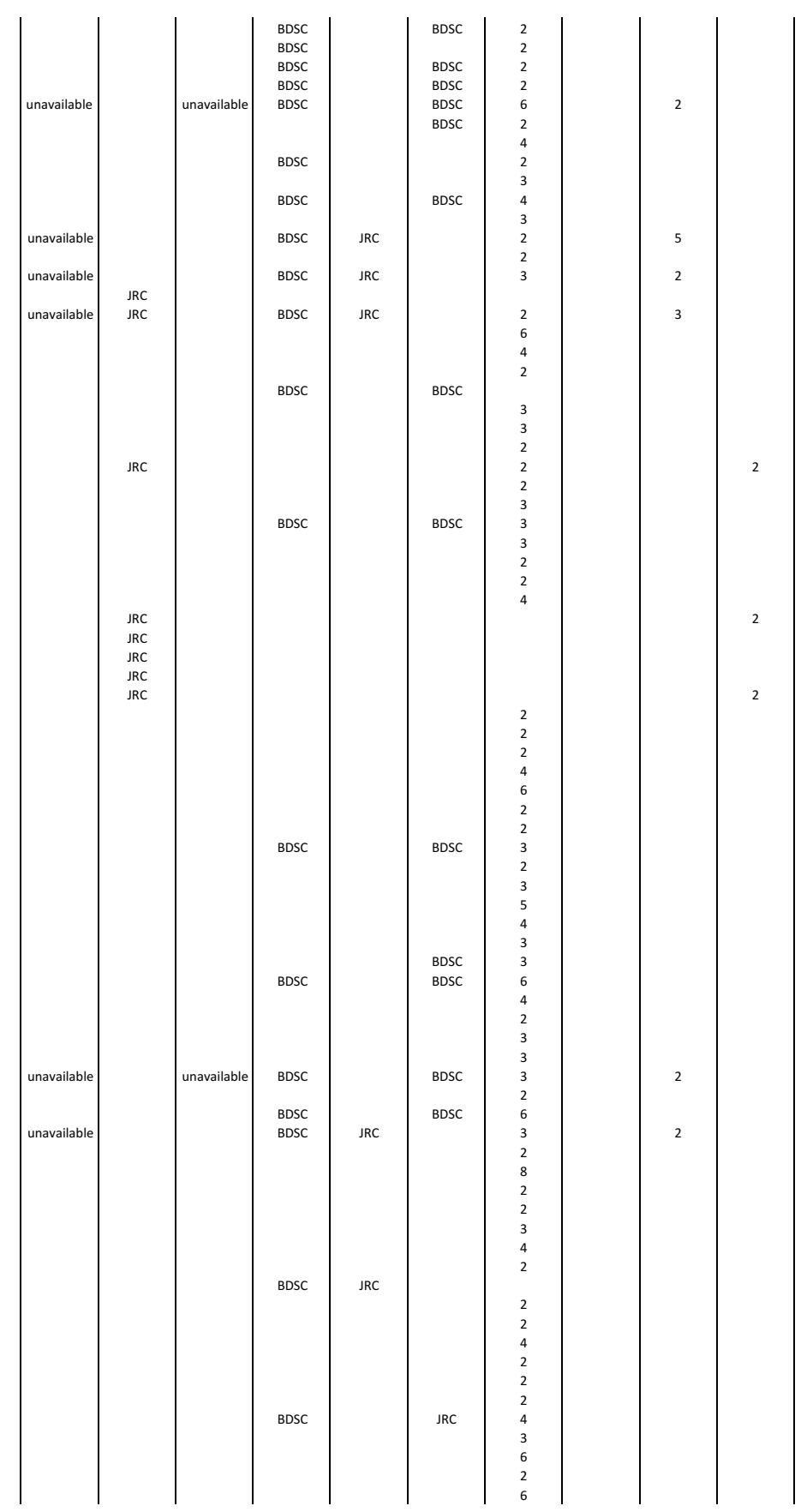


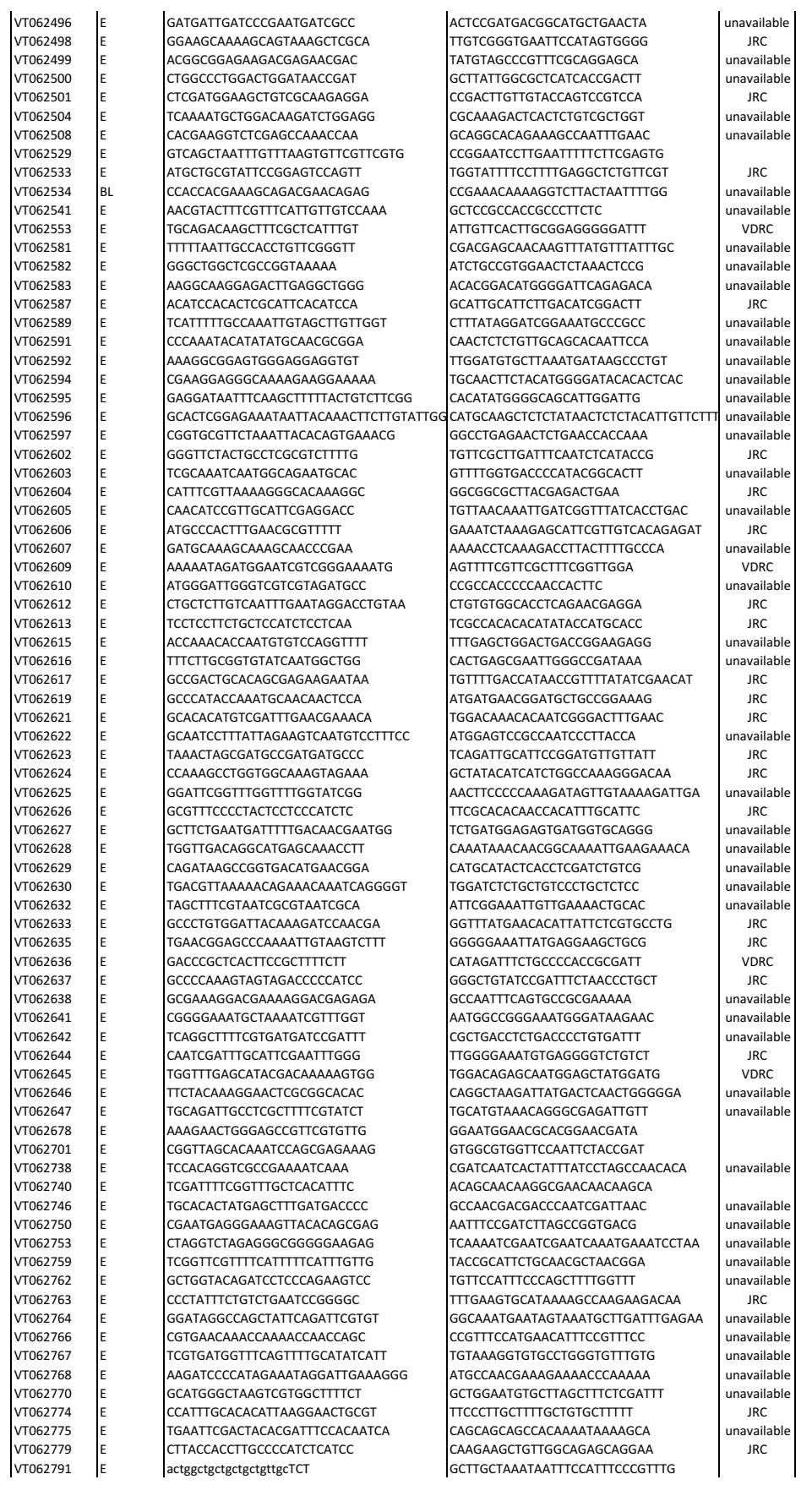
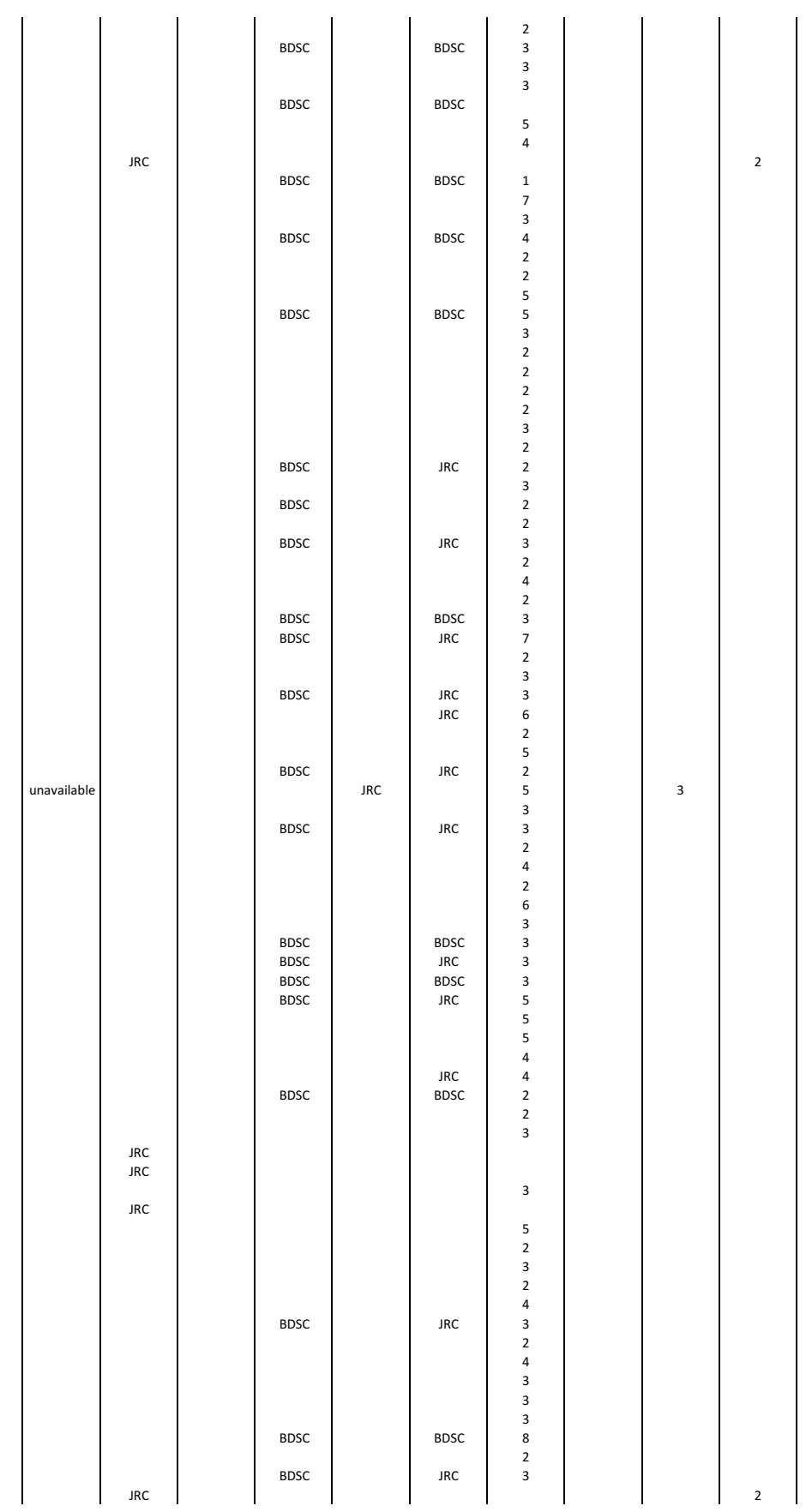


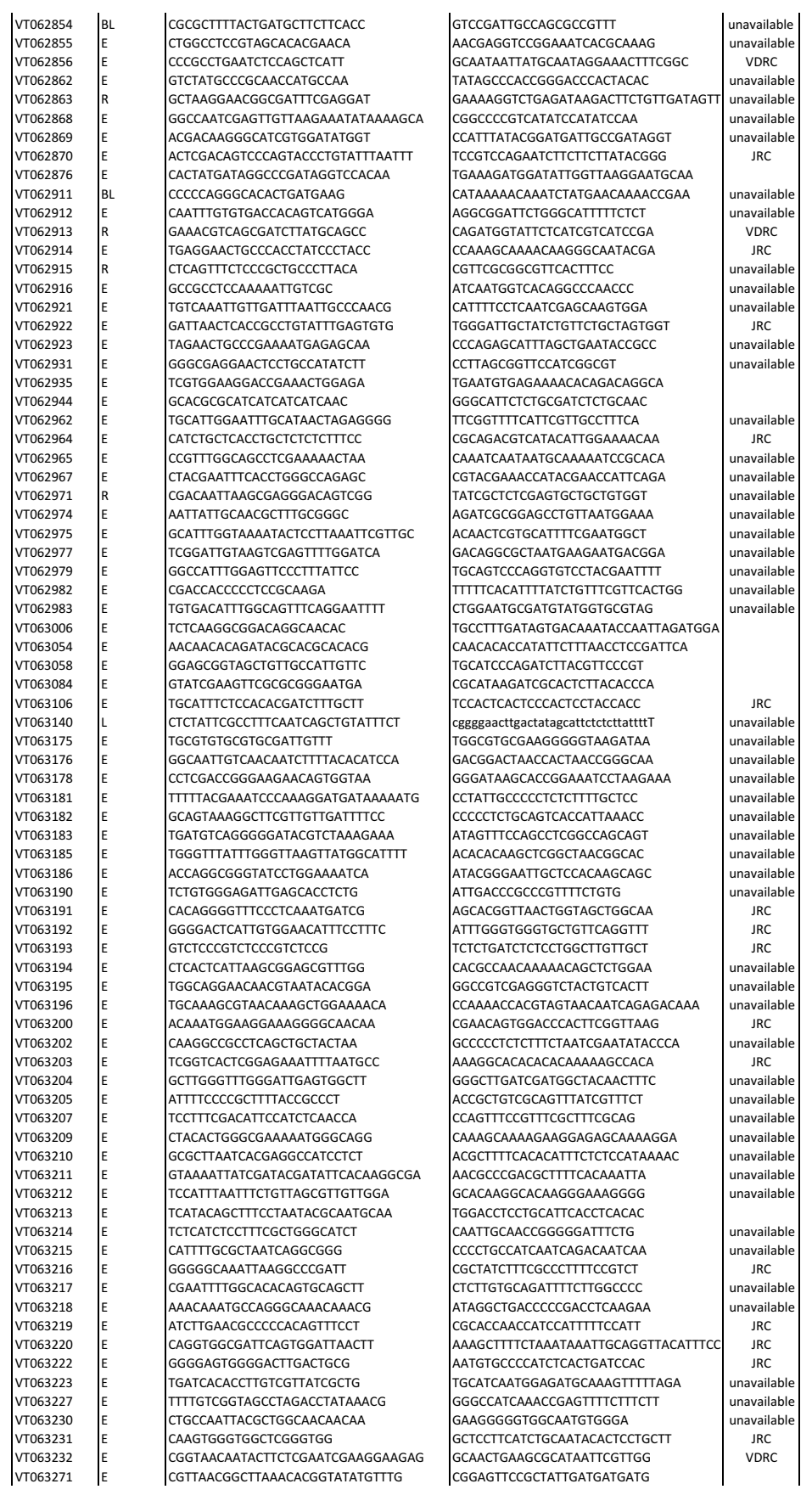
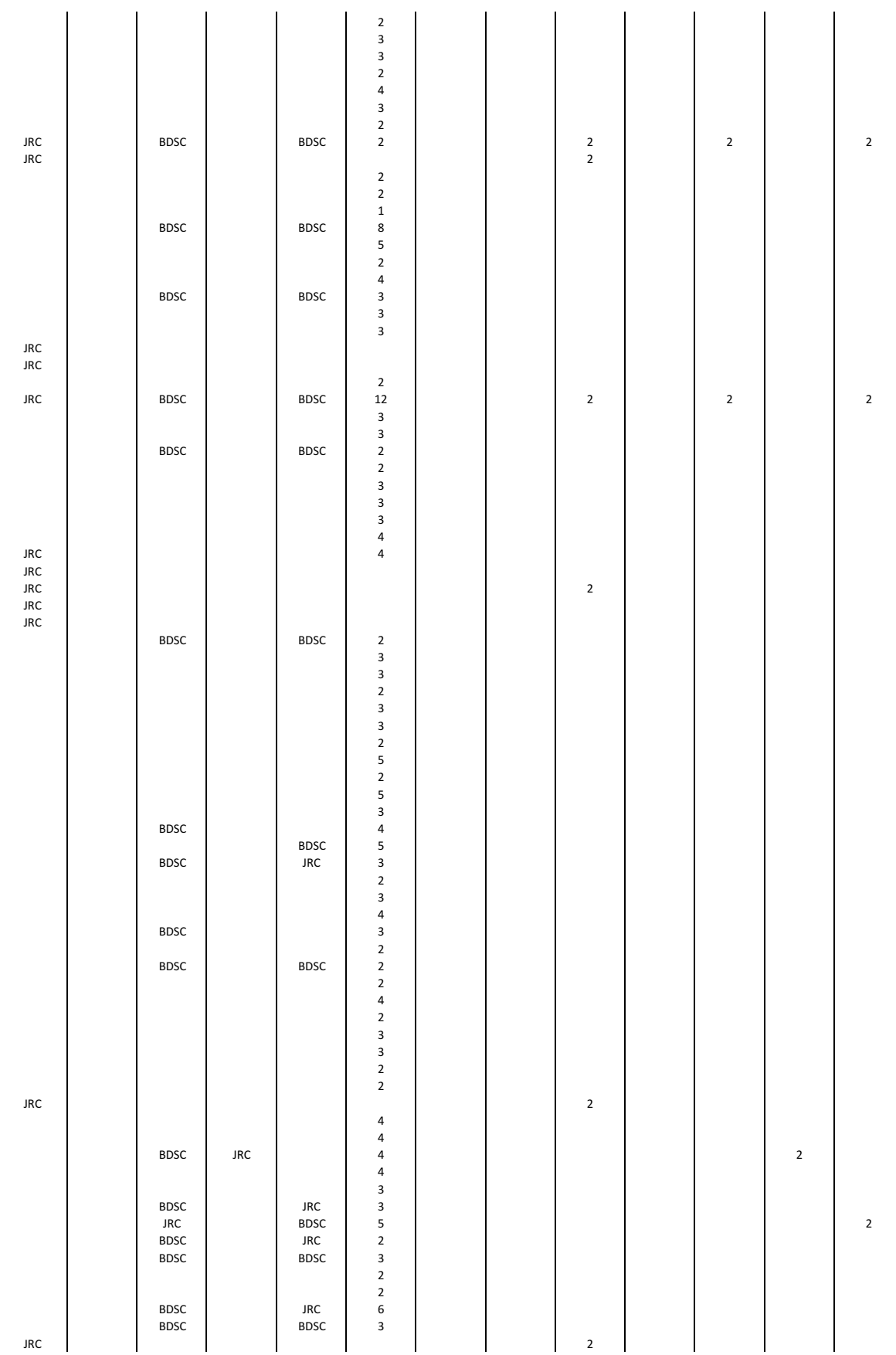


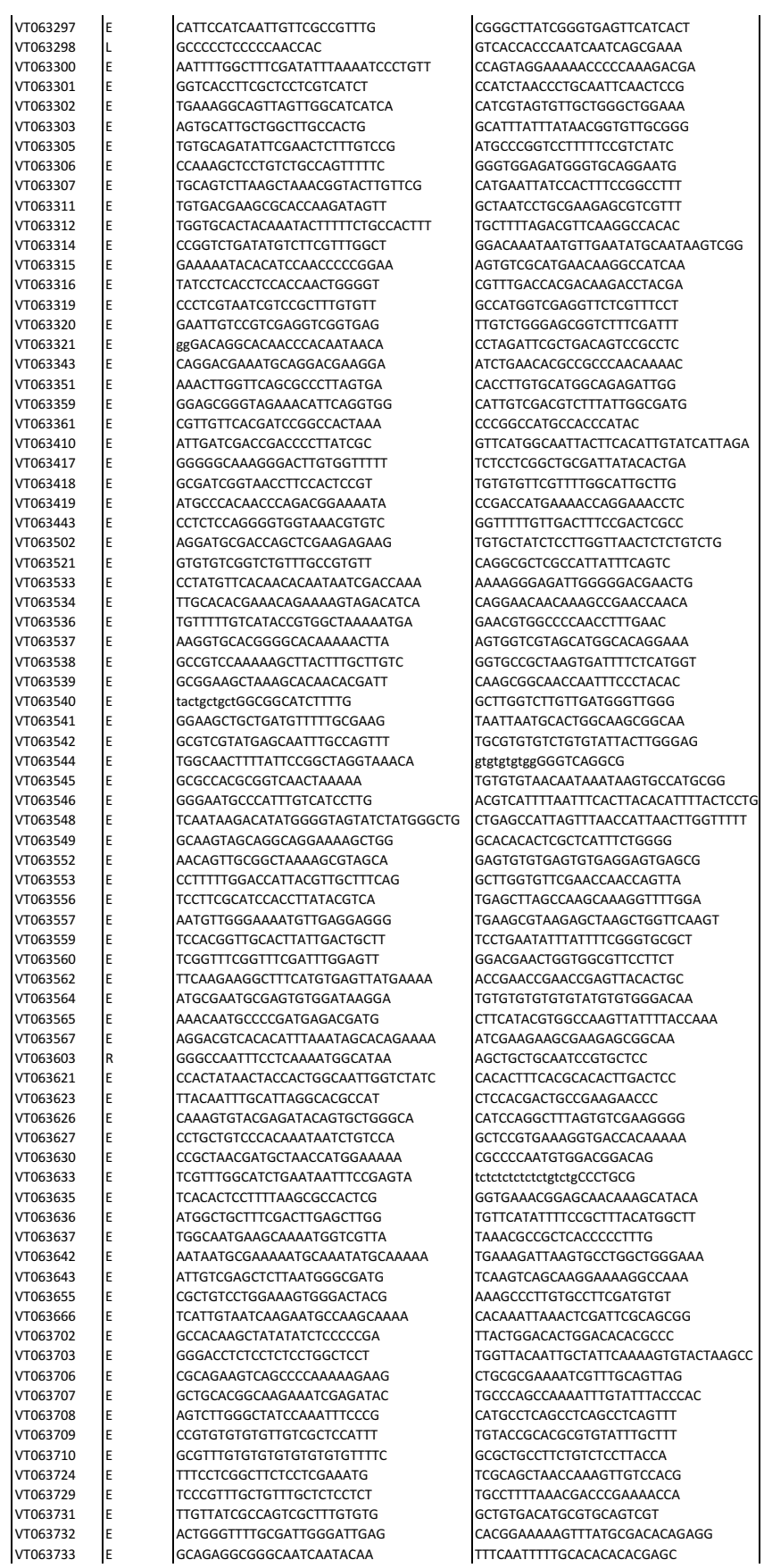
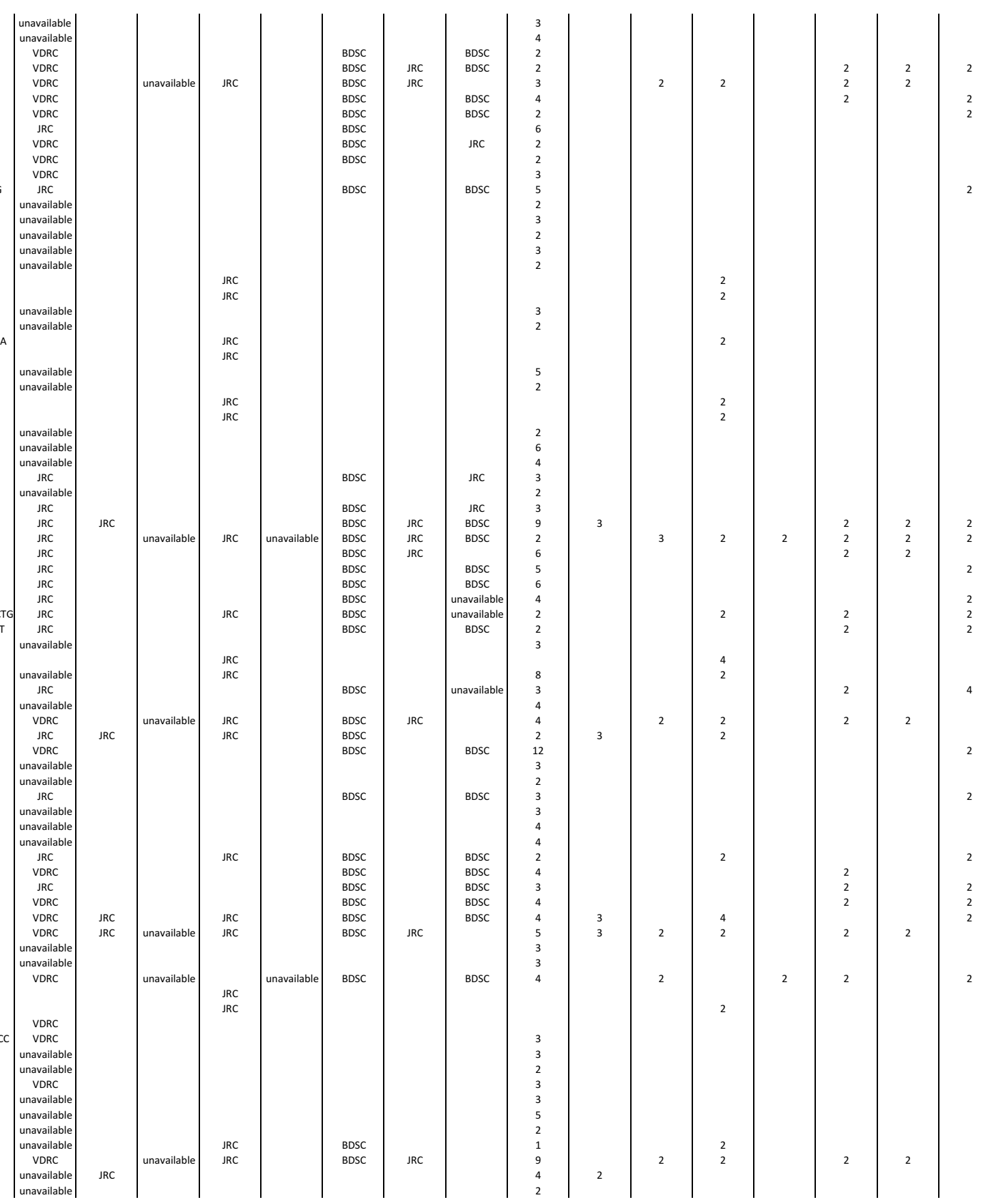


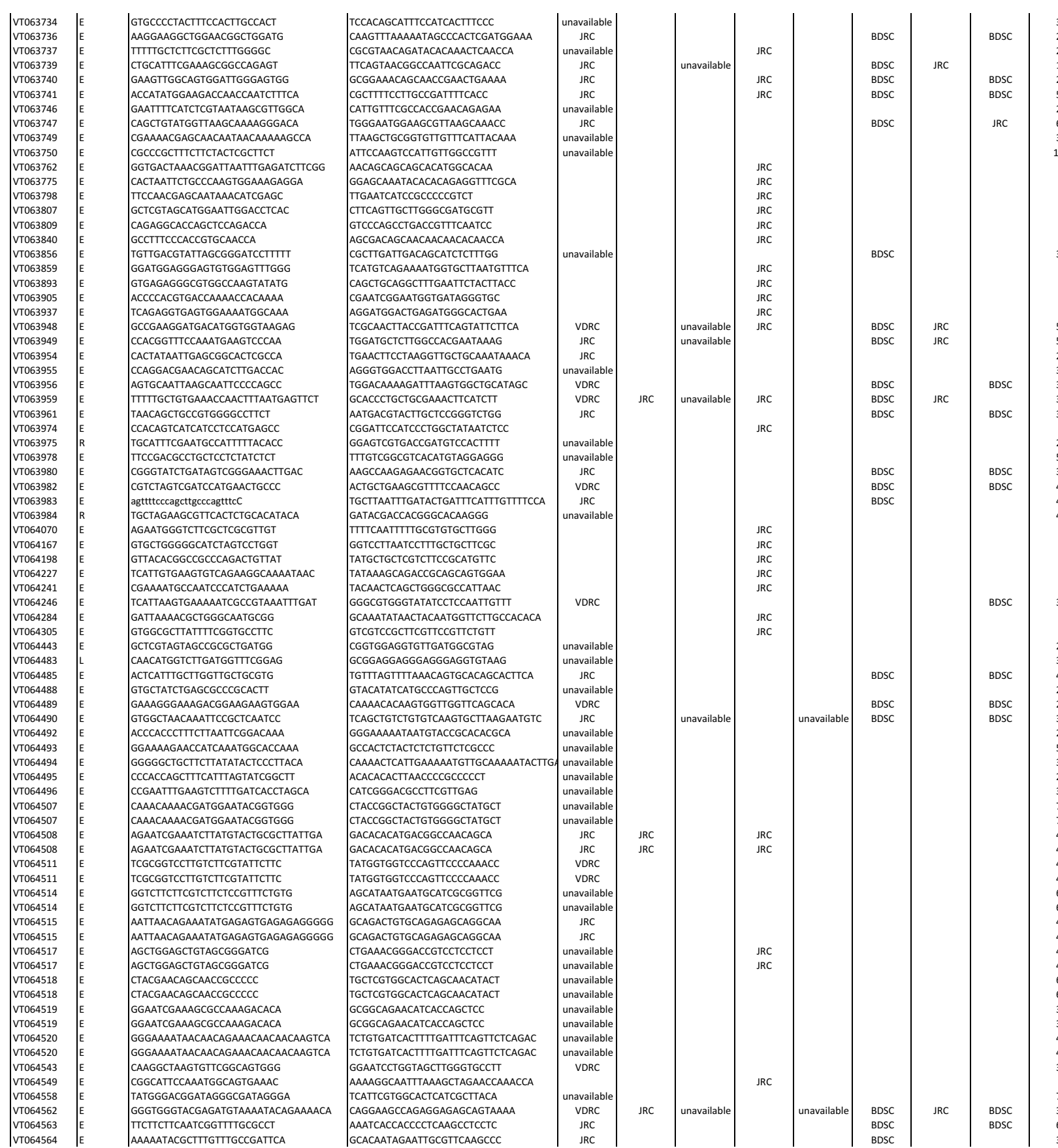




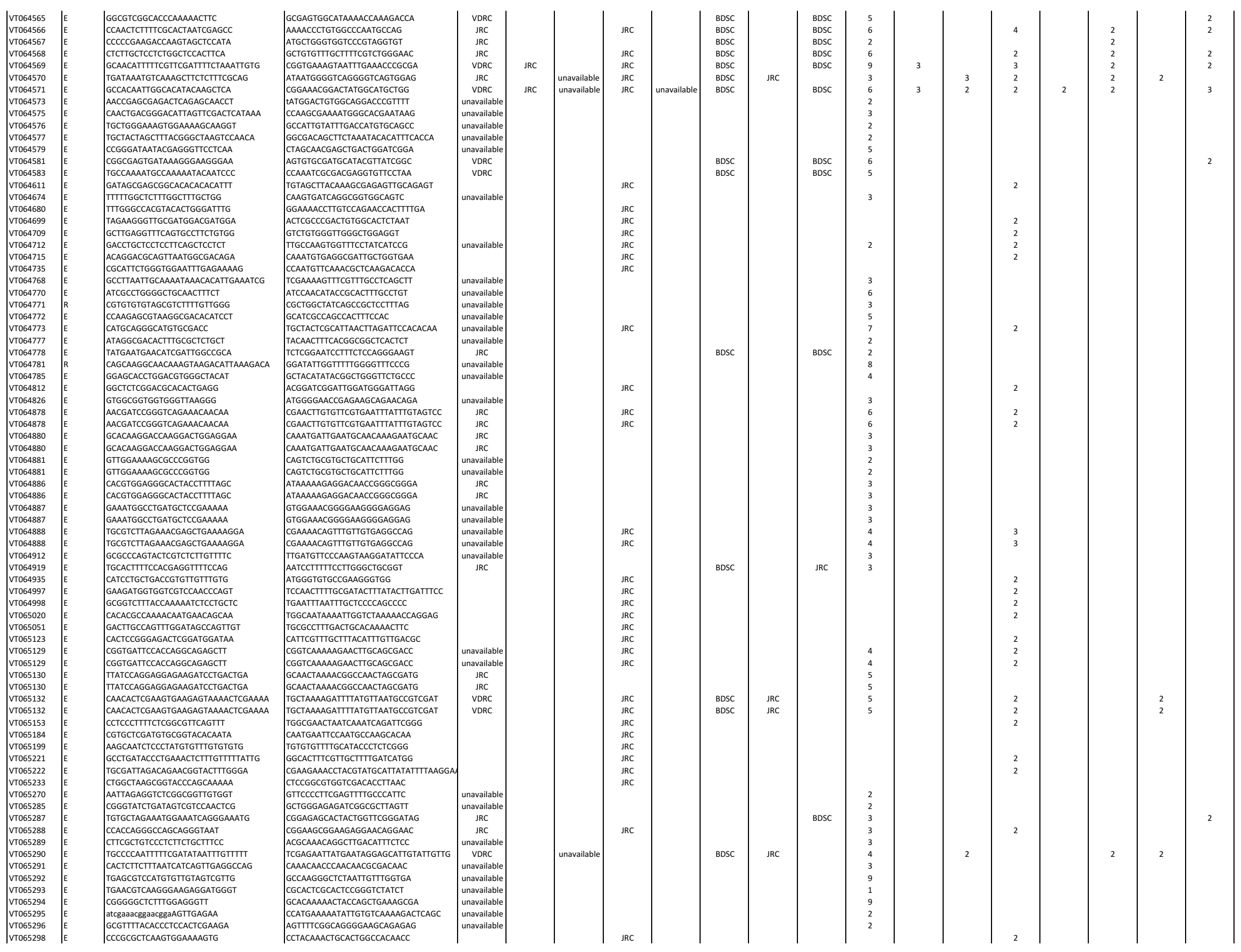




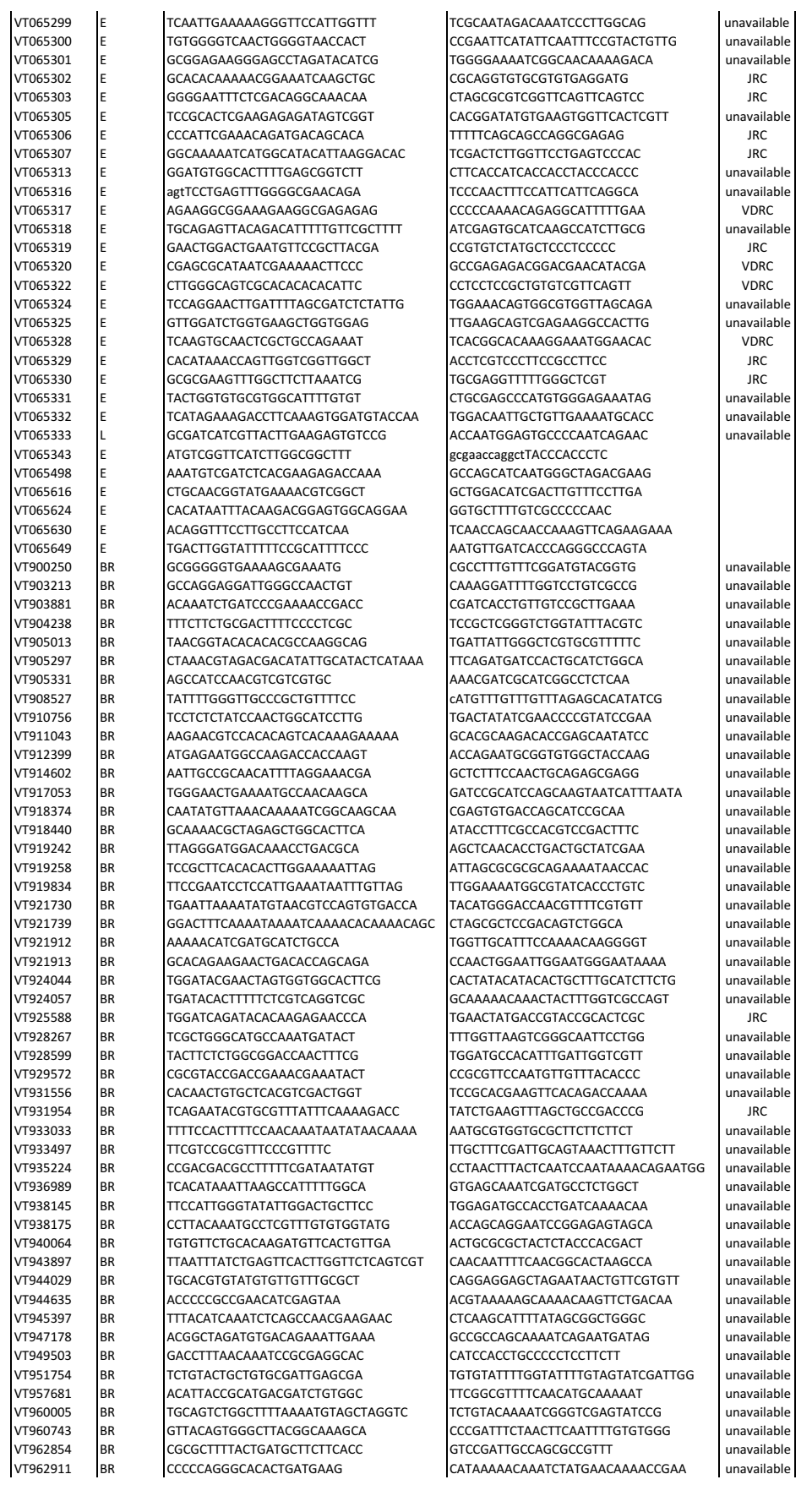
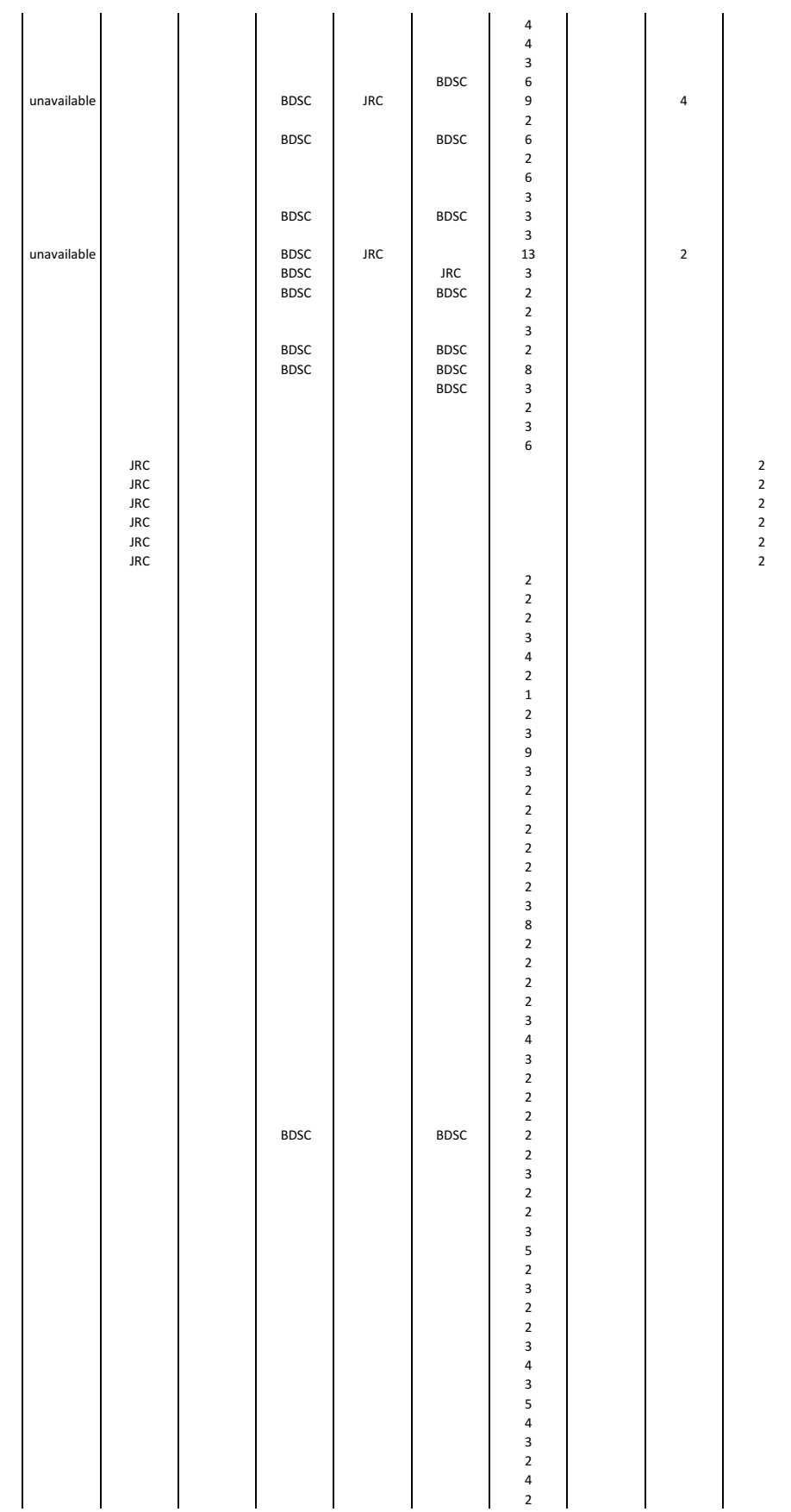


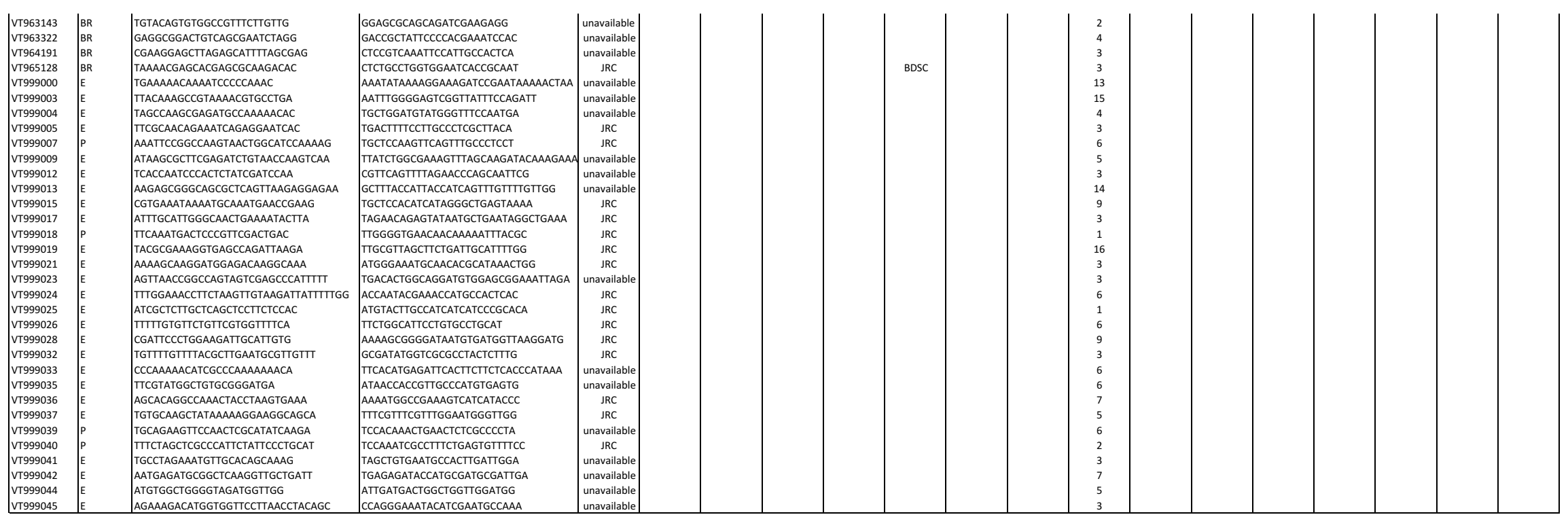

